\title{
Client/Server Based Statistical Computing
}

\author{
D I S S E R T A T I O N \\ zur Erlangung des akademischen Grades \\ doctor rerum politicarum \\ (dr. rer. pol.) \\ im Fach Statistik und Ökonometrie \\ eingereicht an der \\ Wirtschaftswissenschaftliche Fakultät \\ Humboldt-Universität zu Berlin \\ von \\ Herr Dipl.-Kfm. Heiko Lehmann \\ geboren am 26.06.1970 in Berlin
}

Präsident der Humboldt-Universität zu Berlin:

Prof. Dr. Jürgen Mlynek

Dekan der Wirtschaftswissenschaftliche Fakultät:

Prof. Michael C. Burda, Ph.D.

Gutachter:

1. Prof. Dr. Wolfgang Härdle

2. Prof. Oliver Günther, Ph.D.

eingereicht am:

02. April 2004

Tag der mündlichen Prüfung: 12. Mai 2004 


\section{Abstract}

In today's world, many statistical questions require the use of computational assistance. Our approach, presented in this thesis, combines the possibilities of the powerful statistical software environment XploRe, with the advantages of distributed client/server applications, and the opportunities offered by the Internet. In order to offer the client access to a large community, the Java language is used to implement the client's functionalities. The result is a statistical package - usable via the World Wide Web - that can be used like a traditional statistical software package, but without the need for installing the entire software package on the user's computer.

This thesis provides an overview of the desired software environment, and illustrates the general structure with the implementation of the XploRe Quantlet Client/Server architecture. It also shows applications, in which this architecture has already been integrated.

\section{Keywords:}

Client/Server Architecture, XploRe, Java, Statistical Computing, Electronic Books, Interactive Teaching 


\section{Zusammenfassung}

Viele statistische Problemstellungen erfordern in der heutigen Zeit den Einsatz von Computern. Der von uns in dieser Dissertation vorgestellte Ansatz kombiniert die Fähigkeiten der statistischen Softwareumgebung XploRe, mit den Vorteilen einer verteilten Client/Server Anwendung und den Möglichkeiten, die sich durch das Internet eröffnen. Um den Client einer großen Gruppe von Anwendern zugänglich zu machen, wurde Java zu seiner Realisierung verwendet. Das Ergebnis ist ein Statistikpaket, nutzbar via World Wide Web, das wie ein herkömmliches statistisches Softwarepaket verwendet werden kann, ohne die Notwendigkeit, das gesamte Softwarepaket $\mathrm{zu}$ installieren.

Die vorliegende Arbeit gibt einen Überblick über die notwendige Softwareumgebung und erläutert die generelle Struktur der XploRe Quantlet Client/Server Architektur. Die Arbeit zeigt außerdem Anwendungen, in die diese Architektur bereits integriert wurde.

\section{Schlagwörter:}

Client/Server Architektur, XploRe, Java, Statistisches Rechnen, Elektronische Bücher, Interaktives Lehren 


\section{Acknowledgments}

Before actually starting to work on the XploRe Quantlet Client/Server project, and this thesis itself, acknowledgments in papers often seemed somehow strange to me. Now that I went through the entire process myself, I have revised my attitude. It takes much more than just a couple of well-known colleagues to achieve one goal.

First of all I would like to thank my family - my wife Manja and my son Timo - for their patience with me and for supporting this project over the last years.

I would also like to express my gratitude to my adviser Prof. Dr. Wolfgang Härdle, who always supported the development of this project with his thoughtful advise and fruitful discussions.

In the same respect, I would like to extend my appreciation to Prof. Oliver Günther, Ph.D., for his review and comments during the development of this thesis.

For technical support, I would like to thank the members of the XploRe team - Dr. Sigbert Klinke for his continuous help and development of the XploRe Quantlet Server, Dr. Torsten Kleinow and Jörg Feuerhake for the immense effort they put into the client/server project, and last but not least Dr. Zdenek Hlavka, for his extensive testing and evaluation of the XploRe Quantlet Client.

I would likewise reserve my gratitude to Jennifer A. Trimble, Paul T. Harvey and David C. Trimble, Ph.D. for their valuable help in completing this thesis.

Berlin, April 2004

Heiko Lehmann 


\section{Contents}

1 Introduction 1

1.1 Motivation . . . . . . . . . . . . . . . . . 1

1.2 Structure of this Thesis . . . . . . . . . . . . . . . . 4

2 Client/Server Computing $\quad 7$

2.1 Client/Server Computing - A Closer Look . . . . . . . . 8

2.1.1 Client . . . . . . . . . . . . . . 8

2.1 .2 Server ........................ 9

2.1.3 Middleware . . . . . . . . . . . . . . . . . 9 9

2.1.4 N-Tiered Models . . . . . . . . . . . . . . . . 10

2.1.5 Fat Clients versus Thin Clients . . . . . . . . . 11

2.2 From Client/Server to Distributed Computing . . . . . . . 13

2.3 Web Services . . . . . . . . . . . . . . 16

2.4 Statistical Computing vs. Web Services . . . . . . . . . 18

3 XploRe Quantlet Client/Server Model 21

3.1 XploRe Quantlet Server . . . . . . . . . . . . . . 23

3.2 Middleware MD*Serv . . . . . . . . . . . . . . . . . . 24

3.3 MD*Crypt Package . . . . . . . . . . . . . . . 26

3.3 .1 Structure .................... 27 
3.3.2 Client Communication Process . . . . . . . . . . . 28

3.3.3 Programming a Simple Client . . . . . . . . . . . . 38

3.4 XploRe Quantlet Client . . . . . . . . . . . . . . . . 40

4 XQC in Detail 43

4.1 XQC in Action . . . . . . . . . . . . . . . . . 43

4.1.1 Application versus Applet . . . . . . . . . . 43

4.1 .2 Configuration . . . . . . . . . . . . . 45

4.1 .3 Getting Connected . . . . . . . . . . . 46

4.1.4 Desktop .................... . . 47

4.1.5 XploRe Quantlet Editor . . . . . . . . . . . 50

4.1.6 Data Editor . . . . . . . . . . . . . . 52

4.1.7 Method Tree . . . . . . . . . . . . . 58

4.1.8 Graphical Output . . . . . . . . . . . . . 62

4.1 .9 Special Settings . . . . . . . . . . . . . . 65

4.1 .10 Getting Help . . . . . . . . . . . . . . . 67

4.2 Programming Structure of the XQC . . . . . . . . . . . . . 68

4.2.1 XClient - The Starting Point . . . . . . . . . . . 69

4.2 .2 User Interfaces . . . . . . . . . . . . . . . . 71

4.2.3 Character User Interface - CUI . . . . . . . . . . 72

4.2.4 Graphical User Interface - GUI . . . . . . . . . . . 74

5 XQC/XQS Compared to Other Web Based Statistical Solu$\begin{array}{ll}\text { tions } & 89\end{array}$

5.1 CGI Techniques . . . . . . . . . . . . . . . . . . . . . 89

5.2 "Standalone" Java Applets . . . . . . . . . . . . . . . . . . . . 90

5.3 Java Based Client/Server Computing . . . . . . . . . . . . . 91 
6 Reproducible Research Using the XQC/XQS Technology 93

6.1 Types of Presentation . . . . . . . . . . . . . 95

6.1 .1 Text Only . . . . . . . . . . . . . . . . 96

6.1.2 Graphical Representation . . . . . . . . . . . 96

6.1.3 Reproducibility of Calculated Results and Graphics . . 98

6.1.4 Reproducibility and Interactivity Regarding Data . . . 99

6.1.5 Reproducibility and Interactivity Regarding Data and Statistical Programs . . . . . . . . . . . . . . 99

6.2 Making Research Reproducible . . . . . . . . . . . . 100

$6.3 \quad \mathrm{MD}^{*}$ Book . . . . . . . . . . . . . . . 105

$\begin{array}{lll}7 & \text { Interactive Teaching }-M^{*} \text { Stat } & 109\end{array}$

7.1 Introduction . . . . . . . . . . . . . . . . 110

7.2 Characteristics of $\mathrm{MM}^{*}$ Stat . . . . . . . . . . . 111

7.3 Lecture Units . . . . . . . . . . . . . . . . . . . . . 112

7.4 Additional Information . . . . . . . . . . . . . . 113

7.5 Examples . . . . . . . . . . . . . . . . 113

7.6 Reinforcing Previously Learned Statistical Content _ . . . . 117

7.7 Additional Features of MM*Stat . . . . . . . . . . . 117

7.8 Technical Parameters of $\mathrm{MM}^{*}$ Stat . . . . . . . . . . 118

7.9 User Acceptance . . . . . . . . . . . . . . . . . . . . . . 119

7.10 Related Projects . . . . . . . . . . . . . . 120

8 Conclusions 121

$\begin{array}{ll}\text { A License Agreement } & 129\end{array}$ 
B XQC Source Code $\quad 131$

B.1 XClient.java . . . . . . . . . . . . . . . . 131

B.2 XClientAction.java . . . . . . . . . . . . . . 140

B.3 XProperties.java . . . . . . . . . . . . . . 146

B.4 XApplet.java . . . . . . . . . . . . . . . . 150

B.5 XConsole.java . . . . . . . . . . . . . . . . 152

B.6 XEditorFrame.java . . . . . . . . . . . . . . . . 154

B.7 XOutputFrame.java . . . . . . . . . . . . . 156

B.8 XDataMethodFrame.java . . . . . . . . . . . . . . . 158

B.9 XDataMethodAction.java . . . . . . . . . . . . . . 164

B.10 XDataTableModel.java . . . . . . . . . . . . . . . 173

B.11 XDataMethodFrameTree.java . . . . . . . . . . . . . 174

B.12 XDisplayFrame.java . . . . . . . . . . . . . . . . . 177

B.13 XDisplay.java . . . . . . . . . . . . . . . . . . . 179

B.14 XPlot.java . . . . . . . . . . . . . . . . . 182

B.15 XPlotAction.java . . . . . . . . . . . . . . . . 194

B.16 XSetGOpt.java . . . . . . . . . . . . . . . 197

B.17 XReadValue.java . . . . . . . . . . . . . . . . . . . . . 198

B.18 XSelectItem.java . . . . . . . . . . . . . . 200

C Example of a Third Party Client 203

C.1 ThirdPartyClient.java. . . . . . . . . . . . . . . 203 


\section{List of Figures}

2.1 Simple client/server architecture . . . . . . . . . . 8

2.2 Fat Client versus Fat Server . . . . . . . . . . . . . . . . 12

2.3 Communication process using ORPC . . . . . . . . . . 14

3.1 XploRe Quantlet client/server architecture . . . . . . . . . 22

$3.2 \quad \mathrm{MD}^{*}$ Serv structure . . . . . . . . . . . . . . 25

3.3 MD*Crypt structure . . . . . . . . . . . . . . . . 27

$3.4 \mathrm{XQC}$ in action . . . . . . . . . . . . . . . 41

$4.1 \mathrm{XQC}-$ jar file . . . . . . . . . . . . . . . . 44

4.2 XQC started as an applet . . . . . . . . . . . . . . 44

$4.3 \mathrm{XQC}$ - embedded in HTML . . . . . . . . . . . . . . 45

4.4 Manual input for server and port number . . . . . . . . . . 47

4.5 XQC connected and ready to work . . . . . . . . . . . 48

4.6 Console . . . . . . . . . . . . . . . . . . . . . . . . . . . . 48

4.7 Output Window . . . . . . . . . . . . . . . . 49

4.8 Menu 'XQC' . . . . . . . . . . . . . . . . . . . 50

4.9 Menu 'Program' . . . . . . . . . . . . . . . . . 50 50

4.10 XploRe Editor Window . . . . . . . . . . . . . 51

4.11 Menu 'Data' . . . . . . . . . . . . . . . . . . . . 52 


\section{LIST OF FIGURES}

4.12 Dimension of the Data Set . . . . . . . . . . . . . 53

4.13 Combined Method and Data Window . . . . . . . . . . 54

4.14 Working with the Method and Data Window . . . . . . . . 56

4.15 Uploading data . . . . . . . . . . . . . . . . . 56

4.16 Manipulating the uploaded data . . . . . . . . . . . 57

4.17 Uploaded objects . . . . . . . . . . . . . . . . . . 58

4.18 BoxPlot.xpl . . . . . . . . . . . . . . . . 60

4.19 sample_tree.ini . . . . . . . . . . . . . . . 61

4.20 Extract of the xqc.ini f . . . . . . . . . . . . 61

4.21 Final result of our tree example . . . . . . . . . . . . . . . 62

4.22 Scatter plot for characteristics of Swiss bank notes . . . . . . 63

4.23 Rotating scatter plot showing the context menu . . . . . . . . 64

4.24 Showing the coordinates of a data point . . . . . . . . . . 64

4.25 Menu 'Help' . . . . . . . . . . . . . . . . . . . 67

4.26 Version information . . . . . . . . . . . . . . 68

4.27 XQC structure . . . . . . . . . . . . . . . . . . . 69

4.28 Console . . . . . . . . . . . . . . . . . . . . . . 72

4.29 Editor Window . . . . . . . . . . . . . . . . 73

4.30 Method and Data Window . . . . . . . . . . . . . 77

4.31 Building the Method Tree . . . . . . . . . . . . . . . 79

4.32 Drawing data points . . . . . . . . . . . . . . 83

4.33 Calculating the rotation matrix . . . . . . . . . . . 85

4.34 Calculating the rotated data points . . . . . . . . . . . . 86

6.1 Scatter plots with linear regression and kernel regression estimate . . . . . . . . . . . . . . . . . 997

6.2 Reproducible research . . . . . . . . . . . . . . . . . 102 
6.3 Interactivity regarding data . . . . . . . . . . . . 103

6.4 Interactivity regarding data and program . . . . . . . . 104

6.5 Example of reproducibility generated using MD*Book . . . . 105

6.6 Interactive example . . . . . . . . . . . . . 106

$6.7 \mathrm{MD}^{*}$ Book project . . . . . . . . . . . . . 108

7.1 Three dimensions of teaching statistics . . . . . . . . . . . 112

$7.2 \quad \mathrm{MM}^{*}$ Stat - Table of content . . . . . . . . . . . . 113

7.3 Lecture unit . . . . . . . . . . . . . . . . . . . . 114

7.4 Interactive example . . . . . . . . . . . . . . . 116

7.5 s226i.ini . . . . . . . . . . . . . . . . . . 119 


\section{LIST OF FIGURES}

xvi 


\section{List of Tables}

6.1 Results of a decathlon . . . . . . . . . . . . . 96 


\section{Chapter 1}

\section{Introduction}

\subsection{Motivation}

September 1998 - I started working on my diploma thesis with the theme "Statistical Modeling of Sales Promotions in Supermarkets via Neural Networks". Promotions such as features, flyers and special price offers have a great influence on the market sales of the promoted products. Modeling those actions in order to predict market behavior is an absolute necessity for marketing departments to avoid failure and to receive the best results. The aim of my diploma thesis was a comparison between commonly used classical approaches and neural networks.

Starting my work I was faced with two major problems:

- There exist several papers describing classical approaches for modeling market behavior to promotions. Most of them are not filled with just formulas, but with excellent examples and nice graphics. However, locating the programs that had produced those examples in order to use them as a basis for my work was nearly impossible. For me, this meant starting right at the beginning - taking the pure formula and looking for a statistical software environment that I could use to implement the logic.

- This search for a statistical software environment raised problem number two. Most statistical programs were just not affordable for a student at that time. This meant trying to get access to an installed software 


\section{Introduction}

environment at the university, making research and validations at home impossible.

Of course this is just a single example. But as I learned during the last several years - I am not the only one, who faced problems like this.

An enormous number of statistical methods have been developed during the last three decades, e.g. nonparametric methods, bootstrapping time series, wavelets, estimation of diffusion coefficients. To implement these new methods, the developer usually uses a programming environment he is familiar with. Thus, such methods are only available for a certain software package, but not for widely used, standard software packages like MS Excel. To apply these methods to empirical analysis, a potential user may be facing any number of problems. It may even be impossible for him/her to use the methods without rewriting them in a different programming language. Even if one wants to apply the new method to simulated data in order to understand the methodology, he/she is confronted with the same drawbacks. A similar problem occurs in teaching statistics. Since students usually do not have the same statistical software packages as their teacher, illustrating examples have to be executable with standard tools.

In general, two kinds of statisticians are involved in the distribution process of newly implemented methods, the provider and the user. The aim of our work is to close the gap between them. To merge their interests, we introduce a statistical middle ware, a software environment that provides the opportunity to connect user and provider with reasonable effort. For this reason such an environment should have the following features:

1. Sophisticated statistical software including a high level programming language, a developing environment to implement and test methods and a large set of numerical methods.

2. Distribution techniques for new methods, i.e. a mechanism that makes the new method available to many users in a short period of time avoiding any extra effort.

3. An interface for the user that can be integrated in standard software packages in an easy way.

4. Documentation tools. 


\subsection{Motivation}

Several statistical programming environments provide features 1 and 4, e.g. Gauss and XploRe.

If we formulate the second feature for data instead of methods, database servers are supporting it. Data stored in the database is immediately available to all clients of the database server without any distribution effort. This feature is based on the client/server model of database applications. We propose to apply the same technology to statistical methods. This leads us to a client/server architecture, where new methods are stored in a methodbase on the server. In addition we propose to enhance the server with the ability to execute the methods, which shifts the computational burden from the client side to a powerful server. Both aspects will lead to the combination of a methodbase and a powerful statistical engine, and to the use of this combination as a statistical computing server.

The user interface is the client part of the client/server architecture. In order to offer statistical methods to a large community of users, Java applets, which allow for integration into Web browsers, can be used. They are platform-independent and modern browsers already support them without the need for installing additional software. It is the flexibility of Java that makes it useful for teaching statistics. Many Java applets are already available to illustrate statistical content. But most of these applets are primarily developed for particular tasks, e.g. visualization. To extend their features, new Java programs have to be created. In order to combine the advantages of Java with those offered with the client/server concept, we propose to implement the client in Java. Our Java client also supports interactive teaching of statistics as illustrated by Rönz et al. [RMZ00].

Besides the client described in this thesis, the client/server technology of XploRe was used in the GraphFitI software developed at LudwigMaximilians-Universität München [Bla00]. GraphFitI (http://www.stat.unimuenchen.de/) is a Java program that provides model selection methods in graphical chain models. It uses the XploRe Quantlet Server for statistical computation.

In addition to Java, our general concept allows for the implementation of other clients that integrate methods stored at the server into standard software. An example of a non-Java client is the ReX project described by Aydinli et al. [AHKS01], which integrates the statistical engine into MS Excel.

To summarize our approach, the fundamental concept of client/server com- 


\section{Introduction}

puting is the separation of a large piece of software into its constituent parts, thus creating the opportunity for easier development (for our purposes, to integrate new statistical methods) and better maintainability, to facilitate clients and servers running on the appropriate hardware and software platforms for their functions. The client/server architecture is intended to improve flexibility, interoperability, usability and scalability as compared to common software packages.

\subsection{Structure of this Thesis}

Following the explanations in the previous section, in which we described the motivation that stands behind this thesis, Chapter 2 starts with an introduction of the common client/server concept. The basic components of this architecture are described in more detail. We also explain different needs and possibilities that go along with modeling a client/server based application. Furthermore, we transition from client/server based computing to distributed computing, and take a closer look at the Web Service concept.

Chapter 3 describes the basic architecture of our XploRe Quantlet Client/Server model. It begins with a short description of the components that are working on the server side - the XploRe Quantlet Server (XQS) and the middleware $\mathrm{MD}^{*}$ Serv. Next, we take a closer look at the MD*Crypt package that provides the communication protocol within our architecture. Finally, we describe the client communication process in more detail. Working through Chapter 3 should enable a potential user to program his/her own client in order to access the XploRe server.

The aim of Chapter 4 is to enable a user to access the complete functionality offered by the XQC. This chapter contains a detailed description of the architecture's user interface, the XploRe Quantlet Client (XQC) and is divided into two parts. First we describe the XQC from a user's point of view; the functionalities behind the single components, menus and icons that are part of the XQC are explained in detail. A simple sample guides the user through the process of setting up a Method Tree - the heart of the XQC's graphical user interface for communicating with the XploRe server. Available configuration files are also explained in detail. Within the second part of Chapter 4, we go into the programming structure of the XploRe Quantlet Client. We distinguish between the XQC's character user interface on one hand, and its 


\subsection{Structure of this Thesis}

graphical user interface on the other. The realizations of special Java classes, basically those that form the components of the XQC, are explained in detail.

In Chapter 5, we compare our Java client/server model for statistical computing with other approaches, such as CGI techniques and "standalone" Java applets. We also try to work out their advantages and disadvantages.

Chapter 6 describes an example for a practical use of our XploRe Quantlet Client/Server architecture. Making research reproducible has become an important issue these days. It helps readers of scientific articles to validate presented content, and to use recently published methods within their own work. The XploRe Quantlet Client is completely programmed using the Java programming language. Due to this characteristic, it can easily be integrated into HTML or PDF content. We describe types of presentations and show how our XQC/XQS architecture can help to achieve reproducibility. The $\mathrm{MD}^{*}$ Book tool - also described in this chapter - can be used for creating electronic documents (e-books, e-teaching documents) out of a $\mathrm{AT}_{\mathrm{E}} \mathrm{X}$ file.

In Chapter 7 we introduce the statistical teach ware package MM*Stat. It is a flexible and applicable tool for teaching and learning statistics in basic studies. An important characteristic of $\mathrm{MM}^{*}$ Stat is its interactive capability. This capability enables the user to study statistical methods using different variables or data sets, and changing the sample size or the parameters of statistical methods. To integrate interactive capability, the XploRe Quantlet Client/Server architecture is used. The XQC runs as a Java applet integrated into MM*Stat's HTML context.

In Chapter 8 we summarize our work. 
Introduction 


\section{Chapter 2}

\section{Client/Server Computing}

Mainframe architectures have dominated the world of computers for a long time. Simple text terminals allowed for interaction between computer and user. The greatest benefits of this architecture were, and in some areas still are, a good performance for the used applications as well as a adequate protection of the data from outside attacks. However, mainframe architectures also have considerable disadvantages, such as relatively low costefficiency and no possibility for the use of Microsoft Windows based applications [Bur99]. Hardware, as well as software, usually consisted of proprietary systems, which were bound to a single manufacturer in many cases.

A client/server architectures was the answer to overcome these disadvantages. It allows enterprises to scale systems in an easier manner and to install new applications and software updates faster, with less effort and expenditure. Users could apply those newly installed components without loss of time. Graphical user interfaces (GUI) made simpler access to software functionalities possible. The entire system architecture can grow along with the enterprise itself. However, the client/server architecture also possesses disadvantages - the security of data being one example.

At the beginning of client/server systems, both architectures, client/server and mainframe, worked "against each other". This has changed to "next to each other" and in many cases, both approaches even work "with one another". Nowadays, mainframe computers are mainly used within the area of enterprise-critical batch and core processes. Mainly financial enterprises, specifically banks, see the mainframe computer as a suitable architecture for administrating common data resources and bank accounts. Client/server 


\section{Client/Server Computing}

systems have found their range of application in business processes where many users access common hard- and software resources over a network.

The following sections give a short insight into the architecture, the functioning and the development of client/server systems.

\subsection{Client/Server Computing - A Closer Look}

As the name "client/server system" implies, this kind of architecture consists of two logical components - a server, which offers a service and a client, which requests a service from the server.

Both components, therefore, form an architecture with distributed responsibilities, similar to a consumer/producer relationship. This relationship is characterized by a customer who asks for goods and/or services. The aim of the producer is to satisfy the consumer's demands accordingly [Lew98].

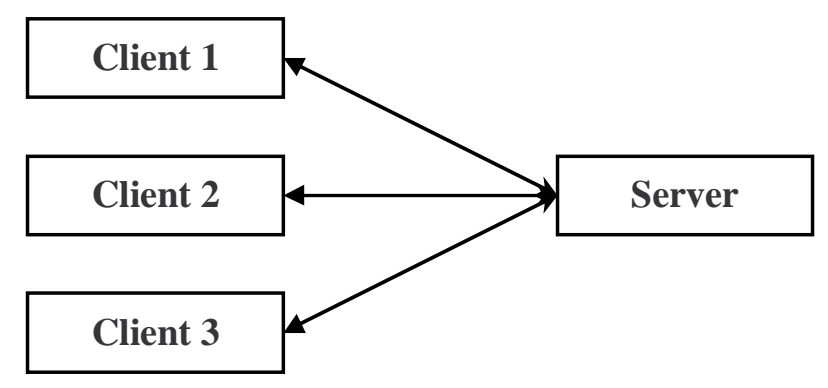

Figure 2.1: Simple client/server architecture

\subsubsection{Client}

A client can be characterized by sending a message to a server, requesting the server to perform a particular task - the service. Clients usually represent the user interface portion of an application, allowing users to get in contact with and use the underlying application. They are responsible of validating data entered by the user, transmitting requests to the server and, in some cases, even executing business logic on the received results. An additional task 
the client has to fulfill is the management of local resources like keyboard, monitor and peripherals.

The number of clients accessing a server is not limited per model definition. Rather, limitations are set by the technical possibilities of the server. The client itself can offer services to other clients acting as a server to those clients.

\subsubsection{Server}

The server is the component in the client/server architecture, which receives the client's requests. Its aim is to solve the requested task and to send the result back to the client.

Solving these tasks can mean implementing complex program logic, accessing database systems and managing large data resources. The result should contain an answer to the client's request. Solving a client's request does not have to be done by the server, which originally received the request. The server itself rather could be connected to other servers. To those servers it can behave like a client, delegating parts of the client's request, or even the complete request to them. No matter how many servers are eventually involved in the processes, at the end of the server's processing remains the fulfillment of the client's request.

\subsubsection{Middleware}

Communication between client and server is made possible by an additional component that is also part of the client/server architecture - the Network Operating System (NOS) or middleware, respectively. The basis for communication processes are known as protocols. These protocols can be divided into three different groups:

- Media protocols,

- Transport protocols,

- Client/server protocols.

A media protocol defines the type of physical connection in a network - examples are Ethernet, Token Ring, and Coaxial. A transport protocol makes 


\section{Client/Server Computing}

the transportation of data packages from client to server and back to client possible. The most common transport protocols are the Transmission Control Protocol / Internet Protocol (TCP/IP) and Novell's IPX/SPX. The client/server protocol comes into action after the physical connection is established and the type of data transmission has been clarified. Client and server must speak the same language in order to be able to communicate with each other. This "same" language is defined within the middleware. The client/server protocol specifies how a client must authenticate itself and in which way service has to be requested. This protocol also specifies how the server must answer client requests in order to guarantee the client understands the results.

Middleware can be grouped according to its task - e.g. to database middleware (SQL, ODBC), Groupware middleware (Microsoft Exchange, Lotus Notes) and Internet middleware (HTTP, SSL).

\subsubsection{N-Tiered Models}

The canonical client/server architecture consists of only two components the client and the server. Due to the number of components involved in this architecture, it is called a "Two-tier" architecture. In this case, the client communicates directly to the server, not taking any "roundabout ways". Application logic can lie either completely on the server's side, completely on the client's side or is split up between both components.

Expanding this architecture with an additional component leads to a socalled "Three-tier" system. The aim of this "third" component can be very diverse. It transitions communication services and/or translation services between client and server, to taking over the complete application logic. In an idealized case, the "third" layer takes over the application logic. Then goal of the client would be a presentation of the data offered by the server (e.g. a database) and processed and/or prepared by the additional application layer. The structure of a "Three-tier" system makes this architecture much more flexible in comparison to a simple "Two-tier" architecture. Running each of the three components on its own hardware allows for an easy scaling of the system, depending on the task for which a component is responsible. A further advantage arises as a result of the separation of data and application layer. The data from different sources can be integrated into the architecture, thereby making that data accessible to the clients. 
One of the most common examples of a "Three-tier" client/server architecture is a standard Web application. A Web browser works as an interface to the user. It forms the first layer - the presentation layer. Modern Web browsers are able to interpret more than just pure HTML. They have the ability to integrate objects such as Java applets or ActiveX components. These browser-integrated components form the middleware, which realizes the application logic - the second layer. The Web server forms the third layer in this example. It supplies the application (e.g. Java applet, ActiveX) with data.

Many of the most recently developed applications are not limited to the use of only three layers. Instead client/server architectures consisting of four, five or even more layers have been developed. Examples of these systems are data warehousing applications consisting of a data repository, a server that unifies the view of the data, an application server that performs queries based on the unified view and a front end that presents the results to the user.

\subsubsection{Fat Clients versus Thin Clients}

Before starting to model a client/server architecture one important question needs to be answered: How should the responsibilities, the intelligence and the authority - how should the application logic be distributed between client and server? The component that utilizes the largest portion of the application logic is characterized as the "fat" part of the model. Conversely, the component that requires less responsibility is called "thin" [OHE96]. As already described in the previous sections, the client usually becomes the component that represents the user interface, whereas the server is responsible for data administration. The distribution of the responsibility for the application logic can be organized in a flexible manner due to the structure of client/server systems. Distributing the application logic between client and server leads to the distinction between "fat clients" and "fat servers".

If the application logic gets shifted to the client side, the resulting model is called a "fat client / thin server" system. One of the most common functions for using these approaches are database systems. A server just administrates the data, whereas clients not only present the data to the user but also process them in certain ways (e.g. performing a statistical analysis). A disadvantage is that updates of the application logic require adjustments of 


\section{Client/Server Computing}

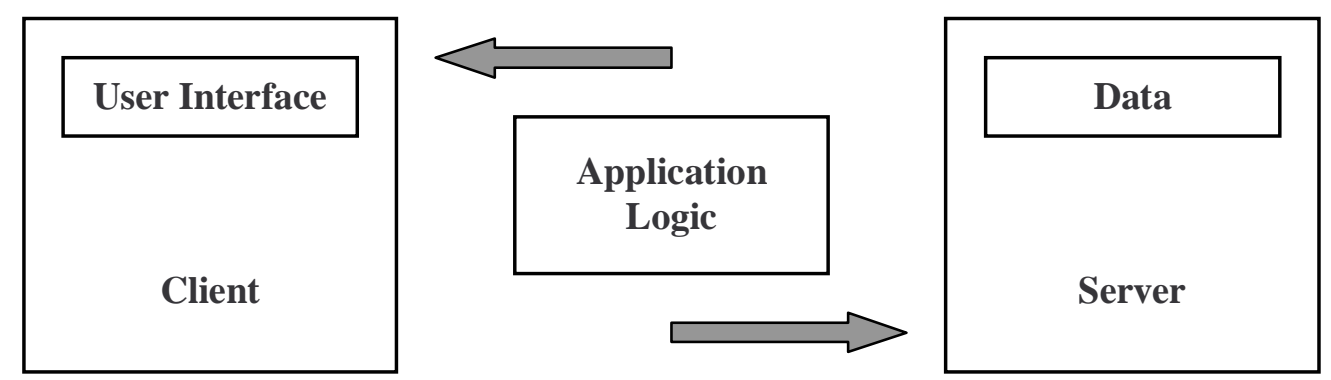

Figure 2.2: Fat Client versus Fat Server

every client in the system. On the other hand, changes on the server side are not compellingly necessary. This enables high stability and integrity of the system. A drawback of this solution is less encapsulation of the data, compared to a "fat server" architecture. The client takes over more logic and more responsibility, which presupposes a deeper knowledge of the data and the way the data are structured and organized on the server side.

Within "thin client / fat server" systems, the application logic lies on the side of the server. Concentrating the application logic on the server side simplifies maintenance and extensions of the system. Software updates require only an update of the appropriate server. Changes on the side of the client are usually not necessary (depending on the kind of update). Often, updates or adjustments can be accomplished without informing the user of the client about the changes at all. As already mentioned before, updating a system where the application logic lies on the client side could result in a change or even in an exchange of every single client. This procedure can be extremely complex and cost-intense, depending on the size of the developed system and/or number of clients in the system. "Thin clients" are a good choice for "task-oriented" processes. These processes are characterized by a client whose major task consists of offering a common user interface for data input and data output. Automatic teller machines, Web server and most enterprise resource planning (ERP) systems are practical examples of this kind of architecture. 


\subsection{From Client/Server to Distributed Com- puting}

The use of client/server solutions is very closely related to the development of object orientation in software development. An object can be viewed as a self-contained entity, which encapsulates data and a set of operations. The operations offered by the object - also called methods - allow for accessing and acting on the data. An object therefore makes possible a separation of data and functionalities from other parts, such as internal logic or sensible data. Objects that exist as standalone objects within a network, independently of other components of the system, are called distributed objects. Other components of the architecture can remotely access the data and methods of distributed objects on demand. The use of standardized message protocols allows for communication between objects of vastly different origin, such as different operating systems or programming languages.

Recalling the characteristics of a client/server architecture and its single components - client, middleware and server - leads to the result that client/server systems actually already bring the best conditions for "distributed computing" along with them. The use of a "fat server" system allows for encapsulation of the data at the server. A client that wants to access this data must use special methods. Results of the methods are not just the "raw data", but data that has already been processed by the server or another component. In this manner the actual "raw data" is invisible and not directly accessible by the client. This form of limited access to the data increases the data security and integrity.

Communication in object-oriented client/server systems is made possible with the use of a protocol framework. The concept of the Remote Procedure Call (RPC) represents one of the most well-known frameworks [BN84]. The aim of this concept is to use a procedure that is running on a remote computer in the same way as it would be running on the local computer of the user. Along with the transition from procedural to object-oriented programming languages, the concept of the remote procedure call also changed. RPC evolved to become ORPC - Object-oriented Remote Procedure Call.

The ORPC's concept is similar to the one on which RPC is based. Instead of accessing the procedures of a remote object, ORPC accesses methods of this object. An object, which allows for interaction triggered by remote components, is called a remote-object. This remote-object is assigned to the 
server-stub-object. On the client side (the component that triggers the interaction), the remote-object is represented by a client-stub-object. This clientstub-object offers the same method signatures as the remote-object. Figure 2.3 illustrates the interaction between all components.

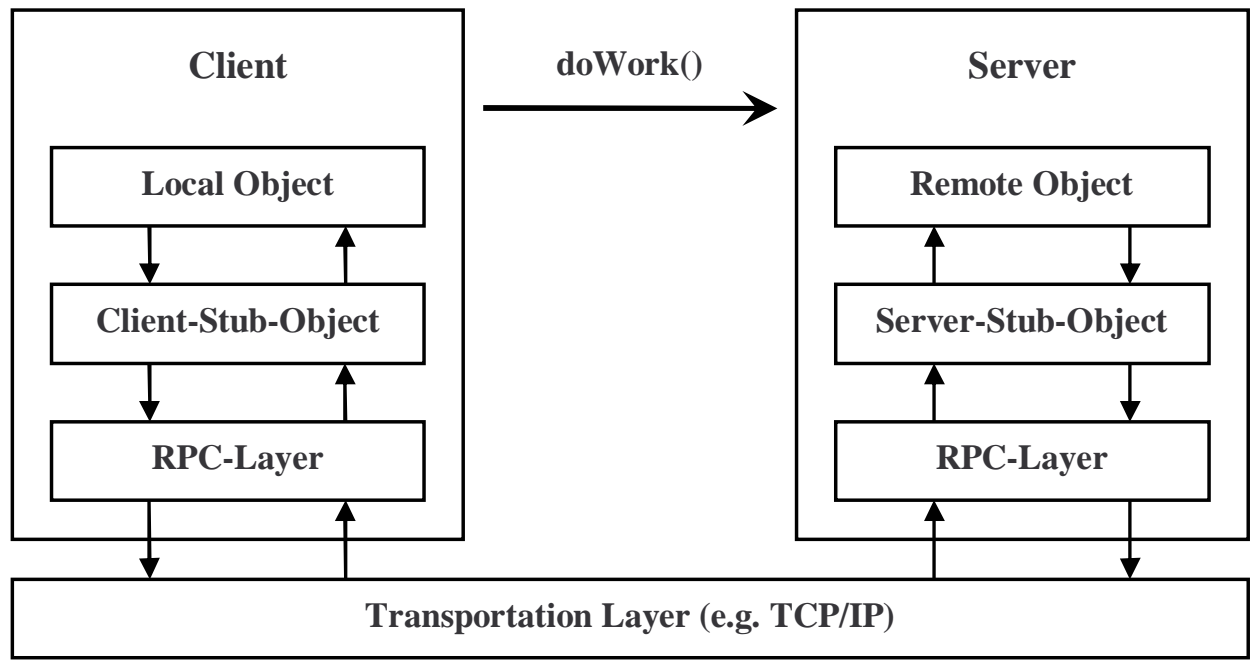

Figure 2.3: Communication process using ORPC

The client uses the client-stub-method to create ("pack") and send a request to the server. The "packed" request is sent to the server-stub-object using a defined transport protocol, like TCP/IP. The server-stub-object unpacks the received information and calls the method on the remote object. The transmission of results back to the client-stub takes places in a similar way [Deg02].

The most common ORPC standards are:

- Common Object Request Broker Architecture (CORBA),

- Java Remote Method Invocation (RMI),

- Distributed Component Object Model (DCOM).

CORBA is the result of development of the Object Management Group (OMG) supported by a consortium of IT enterprises. The aim of this architecture was to work out a common standard, which applies to heterogeneous, distributed systems implemented in different programming languages. 


\subsection{From Client/Server to Distributed Computing}

CORBA defines its own interface description language - the "Interface Definition Language" (IDL) - that works independently of other programming languages. The IDL is used to create client-stub-objects and server-stub-objects in a required programming language (e.g. C, C++, Java) out of IDL files. The most important part of the CORBA architecture is the "Object Request Broker" (ORB). It defines the object model and offers bidirectional locationtransparent object access. The "Generic Inter-ORB Protocol" (GIOP) or for TCP/IP environments "Internet Inter-ORB Protocol" (IIOP) is used as the communication protocol [Sie00].

RMI allows for an object-to-object communication between different Java virtual machines - an RPC in object-oriented Java environments. Using RMI requires a Java platform. Due to this restriction, there is no need for a special interface language. "RMI Wire Protocol" provides the communication protocol for this architecture [Sun04b]. An extension of this protocol - "RMI over IIOP" - offers the possibility to integrate RMI into CORBA environments [Sun04a].

DCOM, introduced by Microsoft with WindowsNT 4.0, was - and still is - meant to be a model for Internet computing, manifesting itself primarily through the use of ActiveX [Mic96, Mic97]. Analogous to the CORBA architecture, Microsoft has developed an "Interface Definition Language" (IDL). It is based on the "Distributed Computing Environment" (DCE), which works as a standard for the classical RPC. Microsoft's IDL is neither CORBA- nor DCE-compliant, which limits the potential for interoperability within other ORPC standards. A further restriction for DCOM results from the fact that this standard is limited to systems running with Microsoft's operating system. Users of alternative operating systems like UNIX, Linux or AppleOS, are not able to use Microsoft's DCOM without additional effort.

The fact that there are at least the three "standards" for distributed computing implies that the "Best Solution" does not exist. Each of the approaches described above is not completely perfect in one way or the other. Their use is limited to a more or less homogeneous environment: DCOM requires a Microsoft environment, RMI requires a Java environment (although Java offers runtime environments for many platforms). CORBA is available on several platforms. However, it presupposes a high measure of adjustments of the client's software to the CORBA architecture. 


\section{Client/Server Computing}

\subsection{Web Services}

In just a little more than ten years, the Internet has evolved from an academic rarity into a powerful tool for doing business. The World Wide Web has become an instrument for exchanging data and information. At the beginning, the Web was a static medium - Web servers provided access to static HTML pages for client browsers that rendered them for display on the user's screen. With the advance of distributed computing and e-Business applications, this model has changed to a more dynamic approach - the contents of the pages to be displayed on the client computer are more often generated on the fly based on the users input and the contents of the business databases [Mau01]. "Interactive Web Contents" and "Web Services" have become common terms in these days.

Searching the Internet for a definition of the term "Web Service" leads to dozens of different results. It has become a term that means many different things to many different people:

- W3C defines a Web Service as “... a software system designed to support interoperable machine-to-machine interaction over a network. It has an interface described in a machine-processable format (specifically WSDL). Other systems interact with the Web service in a manner prescribed by its description using SOAP-messages, typically conveyed using HTTP with an XML serialization in conjunction with other Webrelated standards." [W3C02]

- "Web services are a new breed of Web application. They are selfcontained, self-describing, modular applications that can be published, located, and invoked across the Web. Web services perform functions, which can be anything from simple requests to complicated business processes." [Tid00]

- "... a web service is an application component accessible over open protocols." [Sko02]

Trying to generalize available definitions - Web Services can be viewed as software objects, which communicate with each other via the Internet using standardized protocols. The aim of this communication is to access programs, data, services and resources on distant servers. Bringing it down to a more 


\subsection{Web Services}

abstract definition - Web Services could also be seen as a form of distributed computing between heterogeneous systems via the Internet.

The Simple Object Access Protocol (SOAP) has become a widely used standard for realizing Web Services. Similar to CORBA's communication protocol IIOP, SOAP enables communication between applications that are part of the Internet or Intranet, independently of the underlying platform the applications are installed on. SOAP represents a Remote Procedure Call (RPC) protocol. Up to this point, there is no difference between SOAP and architectures like CORBA, RMI and DCOM.

But SOAP has one feature, which makes it unique when compared to other $\mathrm{RPC}$ standards. It uses an XML-based syntax to wrap information before transmission. A special interface description language - "Web Service Description Language" (WSDL) - makes communication between distributed objects possible. The "Hyper Text Transfer Protocol" (HTTP) is used as the transport protocol. Wrapping all information into an XML file, and using HTTP as the transport protocol, leads to one of the great advantages SOAP offers. It enables SOAP to penetrate Firewalls via the standard-HTTP-port 80. Due to the fact that all information is packed within an XML file, it looks to the server like a common HTML file and can thus pass the Firewall. But this advantage is not undisputable since it also represents a safety-relevant risk. The same standard-HTTP-port-80 method could be used to transport information into Intranets, which is not really welcome (e.g. worms, viruses). It seems to be only a matter of time before Firewalls will close this safety gap by an intensified examination of port 80 .

The XML format that SOAP uses for transportation represents a "double edged sword". The advantage of this format is, without a doubt, the simple legibility of XML files, which simplifies handling (e.g. debugging a transmitted file regarding its form). At the same time, this advantage also represents SOAP's largest disadvantage - it leads to a lack of performance compared to other RPC architectures. The use of XML places a burden on the system and requires additional memory and $\mathrm{CPU}$ resources, due to the requirement to parse and transport XML files [dJ02]. In order to implement a SOAP request, the programmer must generate an XML file, wrap the data to be sent in this file and finally send the file to the server. The answer that arrives from the server is also wrapped within an XML file. This file must be parsed on the client side to extract the data, which again can be quite time consuming. Due to the high overhead of information that comes along with 


\section{Client/Server Computing}

the use of XML, data packages become bigger and transmission takes longer, compared to other RPC approaches.

Practical "Web Service" applications can be divided into three groups [Wai02]:

- Plug-in functionality ...

... represents the simplest form of Web Service by adding third-party functions like stock quotes and search boxes to Web pages.

- Remote infrastructure services ...

... add behind-the-scenes functionalities to Web pages. Online banking services are one of the most common examples for this group.

- Enterprise application integration ...

... offers the possibility to integrate business processes along the value chain.

Major players in this new Web Service landscape are not just common service providers anymore. Rather, the extension of the Internet infrastructure into enterprise has brought businesses from all types of industry into the field of online services. "In the new always-on web service landscape, enterprises are no longer at the end of the value chain. They are part of it, using the shared infrastructure to automate interaction and transaction with their suppliers, employees and customers." [Wai02] Providing online functionalities, enterprises become Application Service Providers - ASP's [GTHM01].

\subsection{Statistical Computing vs. Web Services}

Recalling the information presented in the previous sections one question arises:

"Can distributed computing and the use of Web Services be an appropriate tool to solve the problems we described as part of the Introduction (Chapter 1) in this thesis?"

To answer this question, a closer look at the structure of a common statistical program will help. The aim of a statistical software package is to perform 
statistical tasks on data provided by the user or on sample data offered by the program itself. Computed results will be presented to the user in different ways - pure text, tables, and graphics.

Trying to split a statistical software package into single constituent parts would generally lead to a user interface that represents the computational results, the application itself - fulfilling computational tasks and a collection of data and methods that can be accessed by the application. Hence, we get three different layers. Installing every layer of the software package on a hardware component, which best fits its requirements (e.g. a powerful computer for the actual computation part), results in a classical, distributed "Three-tier" client/server architecture (of course it takes a little bit more than just splitting up existing applications - after all, the single parts have to be able to communicate with each other). If the presentation layer is realized in a manner that allows for an easy integration into World Wide Web content, and the underlying protocols support communication via the Internet, we are more closely approaching a Web Service architecture.

A distributed computing infrastructure of global scale seems to be an important component of the next generation of scientific computing environment [Tod04]. Using the advantages of a distributed computing environment can help to achieve greater computational power, scalability and reusability of data and methods:

- Computational power is an essential need for statistical research. Within a distributed computing environment, a user can access computational power that is not available on his/her local computer.

- Scalability is a result of splitting up the responsibilities between server and client. The actual computing environment can run on a powerful computer, whereas the client can be kept simple in terms of required computing power. Adding new statistical functionalities to the core only concerns the server, not affecting any client.

- Reusability enables users to access data and methods, which can then be distributed on different servers. Newly developed methods can be made available to other users in a very short period of time.

The following chapters describe a client/server approach for statistical computing. We have tried to take advantage of the concepts described in the 


\section{Client/Server Computing}

preceding sections in order to achieve the goals mentioned above. The goal is to enable a user to access statistical computational power and statistical methods with only the need for a common Java enabled Web browser. 


\section{Chapter 3}

\section{XploRe Quantlet Client/Server Model}

The general XploRe Quantlet Client/Server (XQC/XQS) architecture is based on a common three level client/server model as shown in Figure 3.1. It consists of the main components server, middleware and client - see Kleinow \& Thomas [KT00] and Härdle et al. [HKT01].

In this model a server is offering services to one or more client(s). The server of the XQC/XQS architecture consists of the XploRe Quantlet Server (XQS), which represents the powerful statistical engine. For server side communication purposes, the middleware MD*Serv is attached to the XQS.

The server offers access to a database as well as a methodbase, which contain a variety of data and methods. This easily expandable methodbase ensures the possibility of adding newly developed statistical methods and the opportunity to use them via the client without any changes on the client side.

The client is the part of the architecture that requests a service. By using the client, the user is able to access the statistical methods, data and computing power offered by the server. The XploRe Quantlet Client (XQC), responsible for presenting the statistical results, represents the client of the XQC/XQS architecture. For client side communication purposes, the MD*Crypt package is attached to the client. This package implements the MD*Crypt protocol, which is used as the basis for communication between XQC and XQS.

MD*Serv and MD*Crypt handle the communication process between client and server. The choice of communication protocol was based on two issues: 


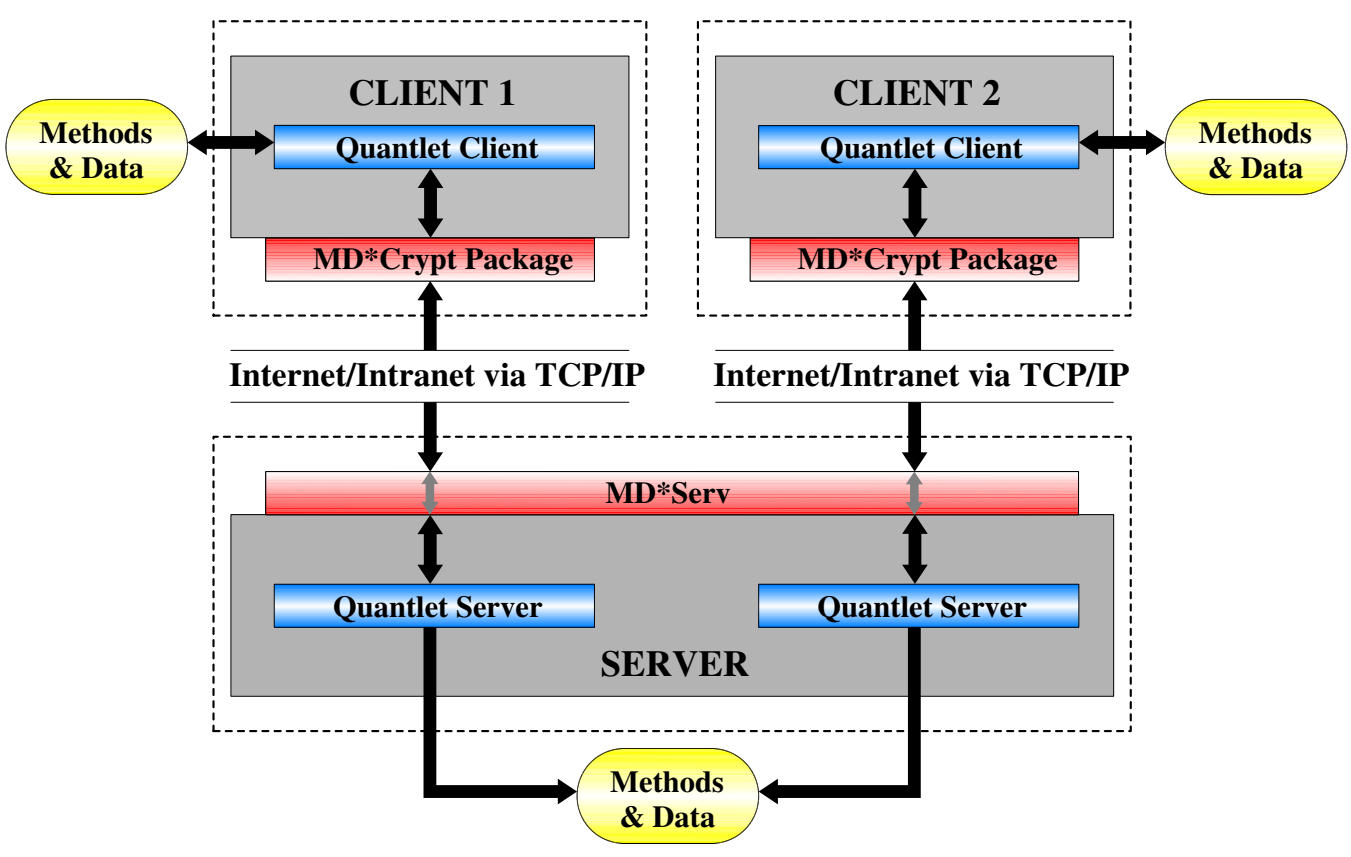

Figure 3.1: XploRe Quantlet client/server architecture

- Statistical computing usually requires a transfer of large data streams from client to server, as well as back from server to client. Keeping the size of data packages as small as possible was a desired outcome of this architecture. Due to their standardization most available protocols like the "Simple Object Access Protocol" (SOAP), require to transfer much more information and data than is actually needed for the pure client's request or server's answers. This reduces the speed of communication between client and server.

- Offering an easy way for client programmers to access the XploRe Quantlet Server is an additional goal of this XQC/XQS project. The programmer should be able to interpret the results of the XQS in a proper and efficient manner. Again, due to their standardization, existing, standard protocols like CORBA and RMI require a close adoption of their underlying architecture. Therefore, they require much more effort by the client programmers.

Having these two objectives in mind, we chose the Transmission Control Protocol / Internet Protocol (TCP/IP) to function as the transport protocol. 
Since the XploRe Quantlet Server communicates on a standard I/O stream which is not appropriate for TCP/IP communication, MD*Serv is utilized to handle the "transformation" between the standard I/O stream and the TCP/IP stream. When transferring data between client and server, it must be guaranteed that client and server are speaking the same language. This task is handled by $\mathrm{MD}^{*}$ Crypt, which defines the client/server protocol.

All components of the XQC/XQS architecture are described in detail in the following sections.

\subsection{XploRe Quantlet Server}

The heart of the client/server architecture is the XploRe Quantlet Server. It represents the back end of the system. The XQS is a powerful computing engine written in $\mathrm{C}++$ that provides a sophisticated statistical programming language. It is based on the statistical computing environment XploRe, which is available for Windows workstations, as well as for UNIX platforms [HKM99]. XploRe provides an extensive set of statistical methods, which are organized in modules:

- Basic Module - basic procedures, data analysis, graphics, mathematics and numerical mathematics, cluster analysis, teach ware,

- Dynamic Systems Module - finance, time series, panel data, errors in variables,

- Flexible Regression Module - generalized linear models, generalized partial linear models, generalized additive models, single index models, hazard regression,

- Non- \& Semiparametric Smoothing - kernel density and regression, kernel estimation, splines, wavelets, neural networks,

- Advanced Statistical Methods - Value at Risk, robust techniques, semiparametric hazard regression, statistical process control.

The XploRe Quantlet Server offers access to these methods. Because the $\mathrm{XQS}$ is written in native code, it enables fast computation. Running on a remote computer, the XQS can offer a high magnitude of computer power, 
which many users would not be able to access by other means. Having access to the method- and database the XQS and the method- and database respectively are easily expandable by new statistical methods via XploRe programs (Quantlets), as well as native code methods, e.g. - $d l l$ and -so. The Communication with MD*Serv is realized via standard I/O streams - the XQS reads from the standard input and writes to the standard output.

\subsection{Middleware $\mathrm{MD}^{*}$ Serv}

MD*Serv works as the server side "/" (slash) in the XQC/XQS architecture. Being the middleware, it facilitates communication between the XQS and possible clients, offering the services of the XQS to the clients. MD*Serv is implemented in Java and relies on the MD*Crypt protocol, which is necessary for the user to access the network services. This protocol dictates the manner in which the client requests services from the server, and how the server replies to those requests.

Due to the design of the client/server model XQS and MD*Serv can be run on a remote host as part of a wide area network (WAN) and/or a local area network (LAN), as well as on the same computer as the client, called local host.

Figure 3.2 visualizes the MD*Serv structure. The MDServ.class contains the main method of the MDServ package. If no specific initialization file is passed to this class via Java's argument feature the file "mdserv.ini" will be used for getting the property information. MDServ.class creates a new instance of the Middleware.class and calls its main method. The Middleware.class serves as the main implementing class. It initiates to read the options of the configuration file (mdserv.ini) and starts needed threads. The configuration file contains information $\mathrm{MD}^{*}$ Serv needs in order to do its work properly:

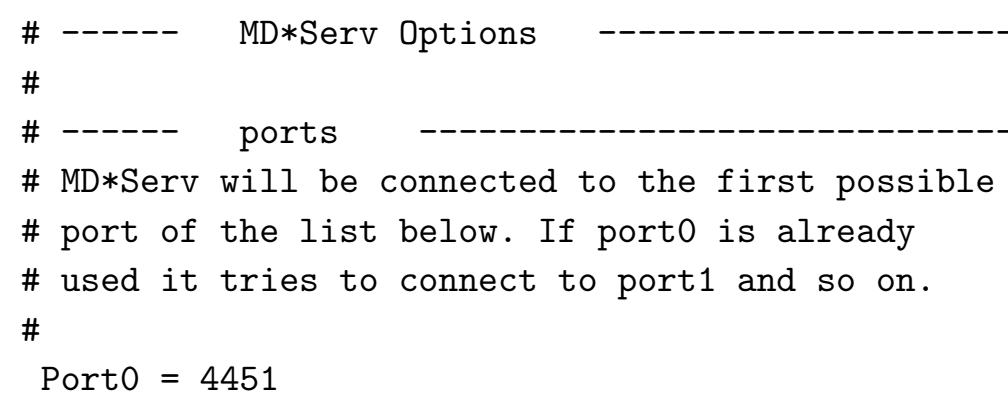




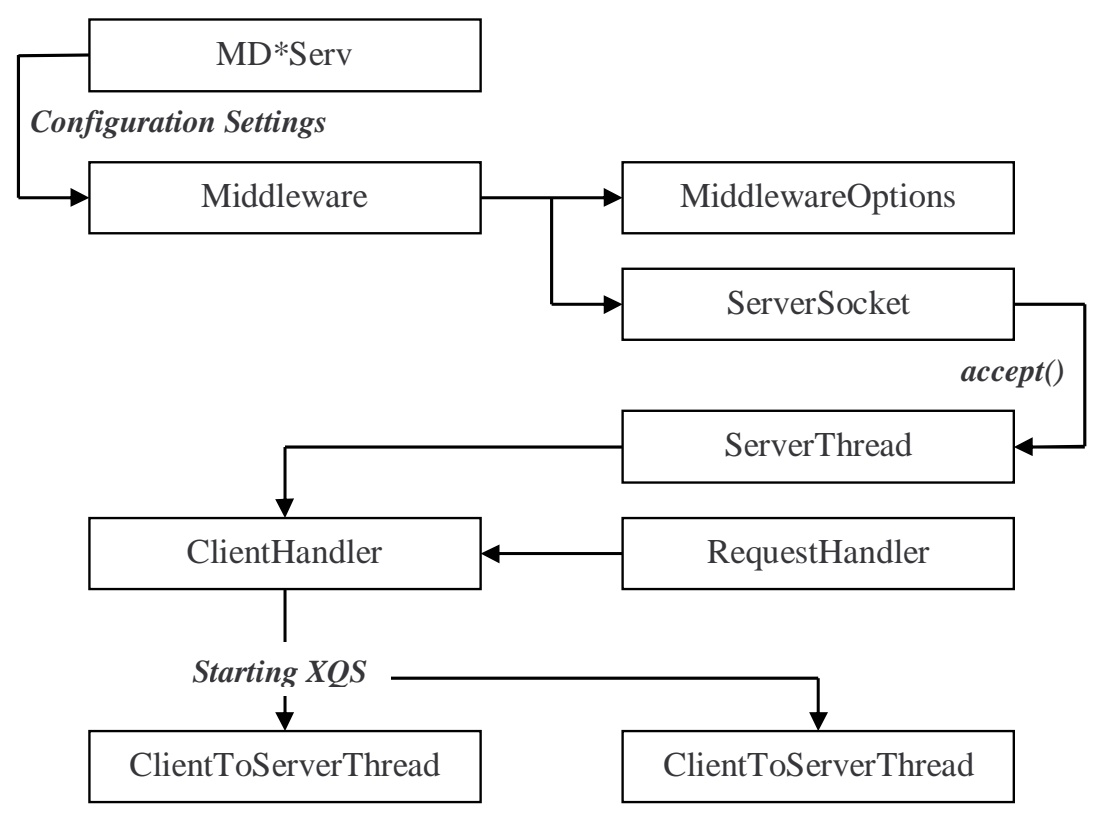

Figure 3.2: MD*Serv structure

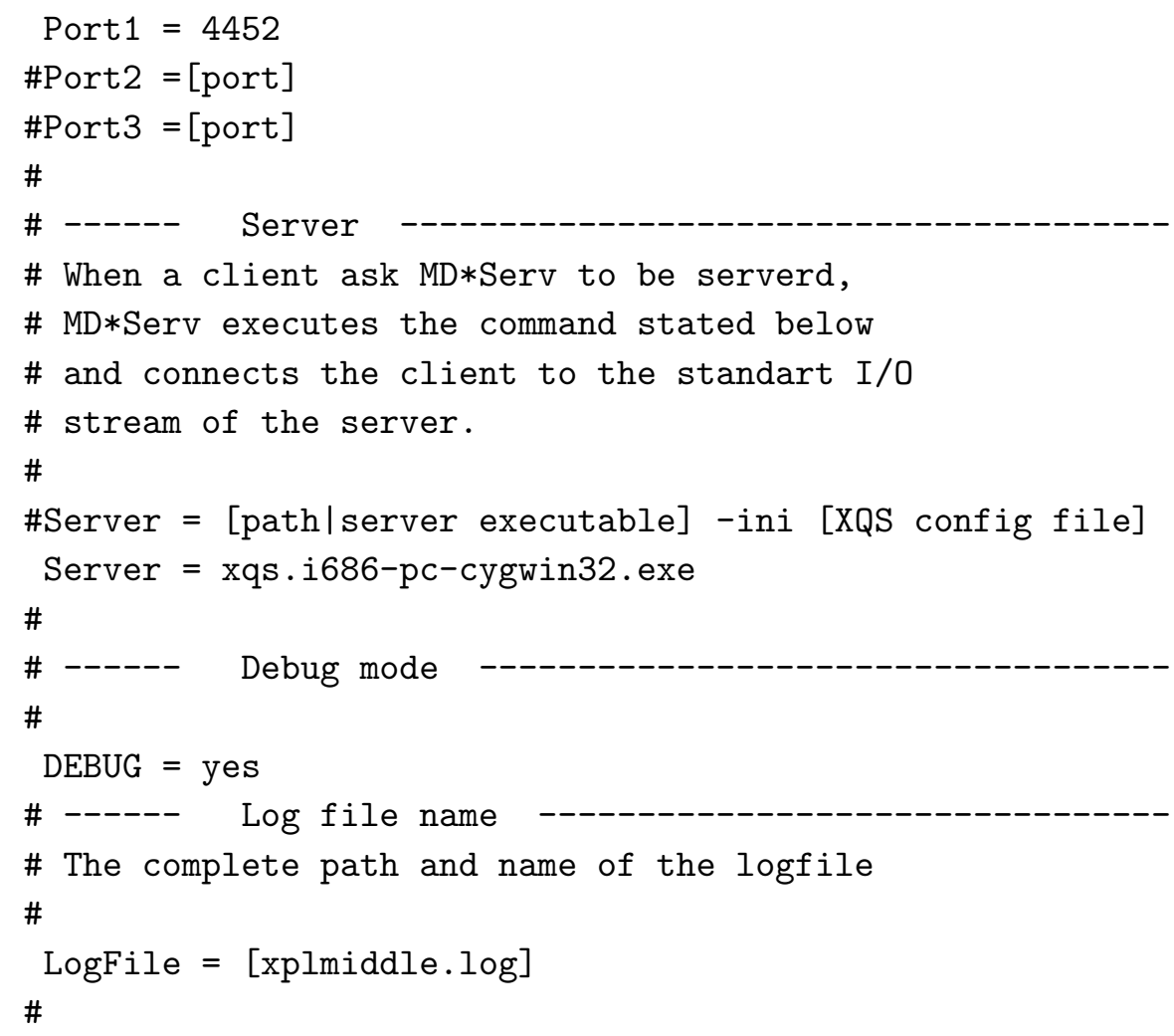




\section{XploRe Quantlet Client/Server Model}

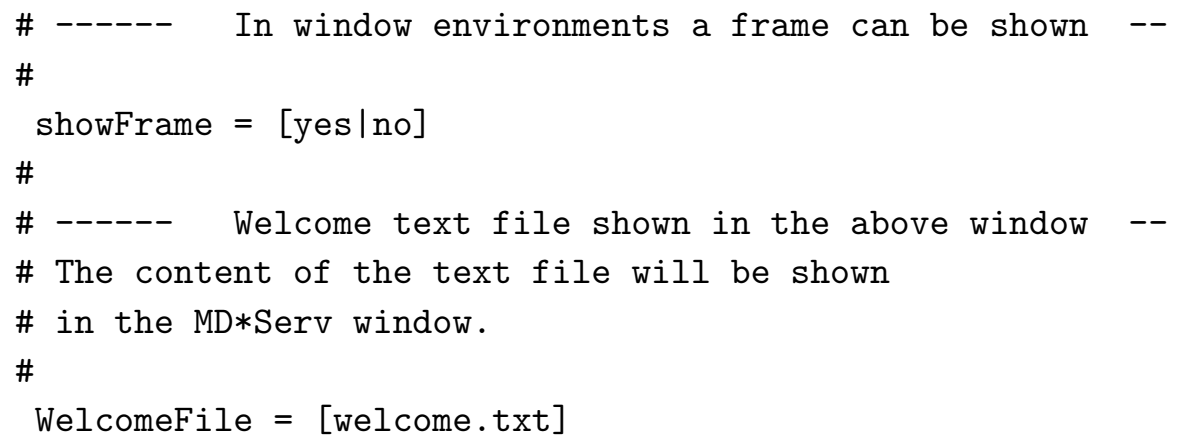

Immediately after the Middleware.class has read all given property information it, attempts to bind a socket to the given specific port on the server. If a successful connection to the port has been established, a new ServerThread will be initiated. This thread listens to the socket and waits for requests. As soon as a request arrives, the ServerThread determines the type and initiates further processing. If a client knows the host name of the machine on which the server is running, and the port number to which the server is connected, it can rendezvous with the server. In the event a client requests a services, a new ClientHandler will be created. This class begins a new session between the requesting client and the XploRe Quantlet Server. It further initializes the data streams and carries out the "handshaking" process. After successfully identifying the client via the arranged protocol, the ClientHandler initiates the XploRe Server batch program, which is defined in the configuration file. This process works exclusively for the requesting client. If the server program was able to start without any problems, the ClientHandler creates two new threads - a ClientToServerThread and a ServerToClientThread. The ClientToServerThread transmits data from the client via TCP/IP to the XQS using its standard input stream. Conversely, the ServerToClientThread transmits data read from the standard output of the XQS to the client via TCP/IP. Since MD*Serv functions as a parallel application, upon connecting requesting client and XploRe Quantlet Server, it is ready for additional (client) requests. In its current version, MD*Serv is capable of handling up to 50 clients simultaneously.

\subsection{MD* Crypt Package}

MD*Crypt works as the client side "/" (slash) in the XQC/XQS architecture. It supports the client side communication between client and server in 
the XQC/XQS model. MD*Crypt is implemented in a single, encapsulated Java package, thereby making it available to different clients, e.g. XQC and GraphFitI, without the double programming effort. Following Java's main argument "write once, run anywhere" it seemed to be the proper language to implement the communication package. Additional reason for Java, to be the language of choice for MD*Crypt, was the "Javadoc" tool, which enables $\mathrm{MD}^{*}$ Crypt to provide class documentation in an instant. Programming skills provided, it is highly adjustable to a wide range of environments. In addition to the Java package there also exists an MS Windows dynamic link library $(d l l)$ for the use in native Windows applications such as MS Excel [AHKS01]. The MD*Crypt package initiates and maintains contact to MD*Serv using the $\mathrm{MD}^{*}$ Crypt protocol. Via TCP/IP, incoming data is made available to the client in an easy accessible manner.

\subsubsection{Structure}

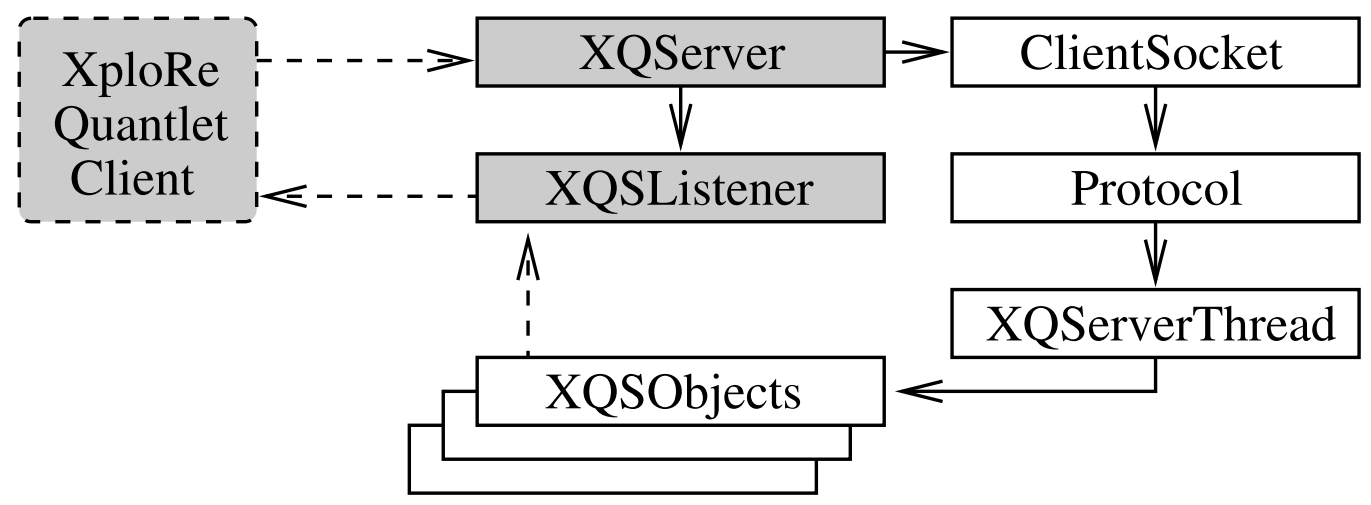

Figure 3.3: $\mathrm{MD}^{*}$ Crypt structure

The MD*Crypt package, being set up in between the XQS (or MD*Serv middleware respectively) and clients, mimics a server to possible front-end clients. Figure 3.3 visualizes the structure of MD*Crypt.

If a potential client wants to communicate with the XploRe Quantlet Server it first of all must create a new instance of the XQServer.class. Containing two different constructors the XQServer can either be started without any parameter - new XQServer() - or with by transmitting a Boolean parameter - new XQServer(boolean debug\{, String logFile\}). The parameter "debug" 


\section{XploRe Quantlet Client/Server Model}

determines whether or not the debugging information will be written on console or in a separate log-file. In order to connect to a specific server and port, this information has to be maintained using the corresponding methods - setServerIP(String server) and setServerPort(int port) - of the XQServer.class.

The connect() method will finally initiate the connection process. Using the given server and port, $\mathrm{MD}^{*}$ Crypt initializes a client side socket that is used for further communication between both applications. The data stream is handled by creating an instance of Java's BufferedInputStream and BufferedOutputStream. Upon successful connection, MD*Crypt attempts to "handshake" with the MD*Serv middleware on the server side. After carrying out a successful "handshake", MD*Crypt and $\mathrm{MD}^{*}$ Serv are ready for the sending and receiving of data and await client requests. The basis for communication between client and server is MD*Crypt's protocol [Feu01]. It defines how the data stream sent to the server has to be structured and and interprets the data stream coming from the server.

The XQServer's method sendQuantlet() allows a client to send XploRe code to the server. Every time XploRe commands have been sent to the server, MD*Crypt initiates a new XQServerThread. The purpose of this thread is to listen for results coming from the server. Using the definitions made within MD*Crypt's protocol class, the XQServerThread processes the incoming data as described below. The thread continues listening until it receives confirmation that the client's request has been completed by the XploRe server.

\subsubsection{Client Communication Process}

Communication between client and server is realized via methods offered by the XQServer.class:

- public void setServerIP(java.lang.String newValue) - sets the IP address of the XploRe Quantlet Server.

- public void setServerPort(int newValue) - sets the port number of the XploRe Quantlet Server.

- public void addListener(XQSListener xqslist) - registers a (client) listener to this XQServer. All results incoming from the server are forwarded to all listeners. 
- public boolean connect() throws java.net.UnknownHostException, java.io.IOException - connects client and server.

- public boolean sendQuantlet(java.lang.String quantlet) - Sends XploRe code to the server. This program code will be executed at the XQS and the result is forwarded to all listeners.

- public void removeListener(XQSListener xqslist) - removes a listener from this XQServer.

- public void terminate() - terminates the connection between XQS and XQC. This method should be executed before terminating the XQC in order to end the XploRe program on server side.

Communication from server back to client takes place via a common Java listener interface offered by MD*Crypt's XQSListener.class. This interface must be implemented by the client. Each client class that has implemented the XQSListener, and is also registered, receives the information regarding content coming from the XploRe server and can thus process it. Implementing means, the client class must define the following three methods:

- serverStatusChanged(int status),

- handleMdCryptException(XQSStatusMessage xqsstm),

- handleServerReply(XQSObject xqsobj).

\section{serverStatusChanged(int status)}

In order to keep the client informed about what is going on during processing, MD*Crypt uses the serverStatusChanged(int status) method. At all times during processing, if the status changes, all listeners registered will be notified. This information can be used by the client to trigger certain events, or just as information for the user of the client. The status information is defined as follows:

- Not connected,

- Socket initialized,

- Handshake done, 
- Connection accepted,

- Server ready,

- Server busy,

- Server waiting.

\section{handleMdCryptException(XQSStatusMessage xqsstm)}

If the XQServer has encountered any problems or exceptions during processing the handleMdCryptException(XQSStatusMessage xqsstm) is utilized to inform registered clients.

\section{handleServerReply(XQSObject xqsobj)}

This method represents the most important method of MD*Crypt's listener interface. It is used to distribute data from the server to registered clients. However, a registered client does not receive the pure and unfiltered data stream. Instead, as mentioned above, MD*Crypt's XQServerThread.class processes the data coming from the server depending on its type. MD*Crypt creates "objects" that contain the interpreted and prepared data. Different types of server returns lead to different object types (e.g. XQSOutputObject, $X Q S D i s p l a y O b j e c t$ etc.). These objects also offer methods to access the data. Creating objects, encapsulating server results and offering methods to access the data, ensures an easy handling of results for the client programmer. A client does not need to be concerned about data stream coding and encoding or interpretation of data. The following section contains a selection of important objects with the corresponding methods provided by MD*Crypt. For a detailed description see http://www.md-crypt.com.

- XQSObject

XQSObject serves as a superclass for XploRe Quantlet server objects. Every $X Q S O b j e c t$ class is derivied from it.

Methods:

public int getType() - returns the type of this XQSObject (e.g. OUTPUT, CREATE_DISPLAY, GRAPHICS, READ_VALUE, SELECT_ITEM, ADD_DATA, STATUS). 
- XQSOutputObject

This object represents a text output object of an XQS. The only data contained in an object of this type is a String.

Methods:

public java.lang.String getText() - returns the text output of an XploRe Quantlet executed on the XQS.

- XQSDisplayObject

This object represents a display object of an XQS. The only data contained in an object of this type are the display id, the number of rows in it and the number of columns.

Methods:

public int getId() - returns the id of this XQSDisplayObject.

public int getCols() - returns the number of columns of this XQSDisplayObject.

public int getRows() - returns the number of rows of this XQSDisplayObject.

- XQSGraphicsObject

This object represents a graphical object of an XQS. It holds the XQSDataObjects, which are meant to be shown within a display, as well as property information about the the display itself.

Methods:

public int $\operatorname{get} \operatorname{Col}()$ - returns the number of columns of this XQSGraphicsObject.

public java.lang.Object getDataObject(int index) - returns the index ${ }^{\text {th }}$ element of DataObjectList. That index must not become larger than the value of $n d p$.

public java.util. Vector getDataObjectList() - returns the complete DataObjectList hold by this XQSGraphicsObject.

public int getDim() - returns the dimension of this XQSGraphicsObject.

public int getDisplayID() - returns the ID of this XQSGraphicsObject.

public java.lang.String getName() - returns the name of this XQSGraphicsObject.

public int getNdp() - returns the number of data parts this XQSGraphic- 


\section{XploRe Quantlet Client/Server Model}

sObject consits of.

public int getRow() - returns the number of rows of this XQSGraphicsObject.

- XQSDataObject

XQSDataObject holds the data and its further point representation. It is used by the XQSGraphicsObject and provides additional methods for reading the data and its properties.

Methods:

public int getDataType() - returns the type of the data. The value of this return can either be 1 , which stands for float data or 2 , which stands for text data.

public int getDim() - returns the dimension of the data.

public int getNumberOfRows() - returns the number of rows of this XQSDataObject.

public double[] getXorgData() - returns the data for the x-dimension.

public double[] getYorgData() - returns the data for the y-dimension.

public double[] getZorgData() - returns the data for the z-dimension.

public double getXmax() - returns the maximum value of the $\mathrm{x}$-dimensional data.

public double getXmin() - returns the minimum value of the $\mathrm{x}$-dimensional data.

public double get $Y \max ()$ - returns the maximum value of the $\mathrm{y}$-dimensional data.

public double get Ymin() - returns the minimum value of the $\mathrm{y}$-dimensional data.

public double getZmax() - returns the maximum value of the $\mathrm{z}$-dimensional data.

public double $\operatorname{get} Z \min ()$ - returns the minimum value of the z-dimensional data.

public double readXvalue (int $i$ ) - returns the $\mathrm{x}$ dimension value at index $i$.

public double readYvalue (int $i$ ) - returns the y dimension value at index $i$.

public double readZvalue(int $i$ ) - returns the $\mathrm{z}$ dimension value at index $i$.

public int getPointPolygon(int i) - returns the polygon which the point at index $i$ should be presented with. The XQS supports 14 polygons to 
represent points graphically:

0 - No graphic representation for this point

1 - Point

2 - Rectangle

3 - Circle

4 - Triangle

5 - 'x'-Symbol

6 - Rhombus

7 - Filled rectangle

8 - Filled circle

9 - Filled rhombus

10 - Filled triangle

11 - '+'-Symbol

12 - '*'-Symbol

13 - Rectangle-grid-symbol

14 - Rhombus-grid-symbol

public java.util.Vector getPointPolygons() - returns a vector of polygons.

public java.awt.Color getPointColor(int i) - returns the point color information of index $i$.

public java.awt.Color[] getPointColors() - returns an array of color information for points of the XQSDataObject.

public int getPointLook(int i) - returns information as to how the point at index $i$ looks.

public int[] getPointLooks() - returns an array with information how points look.

public int getPointSize(int i) - returns information of the point size at index $i$.

public int[] getPointSizes() - returns an array with information of point size.

public java.awt.Color getLine Color(int i) - returns the line color information of index $i$.

public java.awt.Color[] getLineColors() - returns an array of color information for lines of the XQSDataObject.

public int getLineLook(int i) - returns information how the line at index $i$ looks.

public int[] getLineLooks() - returns an array with information how lines look. 


\section{XploRe Quantlet Client/Server Model}

public int getLineSize(int i) - returns information of the line thickness at index $i$.

public int[] getLineSizes() - returns an array with information of the line thickness.

public java.lang.String getPointText(int i) - returns the text of the $i^{\text {th }}$ point.

public java.lang.String[] getPointText() - returns the text of points of this XQSDataObject.

public java.awt.Color getPointTextColor(int i) - returns the point text color information of index $i$.

public java.awt.Color[] getPointTextColors() - returns an array of color information for point text.

public int getPointTextLook(int i) - returns information how point text at index $i$ looks.

public int[] getPointTextLooks() - returns an array of information for point text.

public int getPointTextSize(int i) - returns information of point text size at index $i$.

public int[] getPointTextSizes() - returns an array of information for point text size.

public java.lang.String[] getTextData() - returns the text data to be shown in a display.

- XQSReadValueObject

XQSReadValueObject represents an XQS "read value" dialog. It can hold and modify data, which is determined on client side.

Methods:

public double getValue(int i) - returns the default value at index $i$.

public double[] getValueArray() - returns an array of default values.

public java.lang.String getTextValue(int i) - returns the text value at index $i$.

public java.lang.String[] getTextValueArray() - returns an array of text values.

public void setValue(int $i$, double newValue) - modifies the value at index $i$ 


\subsection{MD*Crypt Package}

to newValue.

public void setValueArray(double[] newValue) - modifies the values to the content of the array newValue.

public void setTextValue(int i, java.lang.String newValue) - modifies the text value at index $i$ to newValue.

public void setTextValueArray(java.lang.String[] newValue) - modifies the text values to the content of the array newValue.

\section{- XQSSelectItemObject}

XQSSelectItemObject represents an XQS "select item" dialog. In this object, the item names are converted to a ListObject rather than an array to make use of the additional properties and services of ListObjects.

Methods:

public java.awt.List getNamelist() - returns the list with values to be shown.

\section{- XQSSetGOptObject}

This class holds properties that are set in the setgopt(..) command of XploRe. Refer to the setgopt() help pages of XploRe to find out what the options mean.

Methods:

public int getId() - returns the ID of this XQSSetGOptObject (equals the ID of a plot).

public boolean getIsXaxis() - returns true if the X-axis shall be painted.

public boolean getIs Yaxis() - returns true if the Y-axis shall be painted.

public boolean getIsZaxis() - returns true if the Z-axis shall be painted.

public java.lang.String getTitle() - returns the title to be printed in a plot.

public java.lang.String getXLabel() - returns the $\mathrm{X}$-axis label to be printed in a plot.

public java.lang.String getYLabel() - returns the Y-axis label to be printed in a plot.

public int getDisplaysizex() - returns the value of horizontal display size.

public int getDisplaysizey() - returns the value of vertical display size. 


\section{XploRe Quantlet Client/Server Model}

As mentioned above, XploRe commands between client and server can be sent by using the XQServer.sendQuantlet(String) method. This means that all information, including data, would have to be sent in a String format. But converting data into a String, then sending the data as a String to the server, is a very time consuming and inefficient method. To solve this problem, MD*Crypt offers objects to efficiently send an receive data sets:

\section{- XQCDoubleVector}

$X Q C D o u b l e V e c t o r$ enables the client to store numeric arrays of dimension one to use it with the XploRe Quantlet Server.

Constructors:

public XQCDoubleVector(double[] array, java.lang.String Name) - constructs a $X Q C D o u b l e V e c t o r$ from the given vector of elements and with a defined name.

Methods:

public int getRows() - returns the number of rows of the vector.

public double getElement(int $i$ ) - returns element $i$ of the vector.

public double[] getElements() - returns a vector of elements.

public void setElements(double[] elements) throws java.lang.Exception - sets the elements of this XQCDoubleVector object.

- XQCDoubleMatrix

XQCDoubleMatrix enables the client to store numeric arrays of dimension "two" to use it with the XploRe Quantlet Server.

Constructors:

public XQCDoubleMatrix(double[][] array, java.lang.String Name) - constructs a two dimensional matrix from the given matrix of elements and with a defined name.

Methods:

public int getCols() - returns the number of columns of the matrix.

public int getRows() - returns the number of rows of the matrix.

public double getElement(int $i$, int $j$ ) - returns element $i, j$ of the of the matrix.

public double[][] getElements() - returns the matrix elements of the $X Q C$ DoubleMatrix object. 
public void setElements(double[][] elements) throws java.lang.Exception sets the elements of this XQCDoubleMatrix object.

- XQCDoubleArray

$X Q C D o u b l e A r r a y$ enables the client to store numeric arrays up to eight dimensions to use it with the XploRe Quantlet Server.

Constructors:

protected XQCDoubleArray(double[][][][][][][][] array, java.lang.String name) throws java.lang.Exception - constructs an array from the given array of elements and with a defined name.

Methods:

public int[] getDimensions() - returns the dimension of the array.

public double getElement(int[] d) throws java.lang.Exception - returns the element at array position[d[0]][d[1]][d[2]][d[3]][d[4]][d[5]][d[6]][d[7]].

To pass and receive these data sets to and from the server, the XQServer.class offers corresponding methods:

- public boolean putDoubleVector(double[] newVector, java.lang.String Name),

- public java.util.Vector getDoubles(java.lang.String objName),

- public boolean putDoubleMatrix(XQCDoubleMatrix Matrix),

- public XQCDoubleMatrix getdoubleMatrix(java.lang.String objName) throws java.lang.Exception,

- public XQCDoubleArray getdoubleArray(java.lang.String objName) throws java.lang.Exception. 


\subsubsection{Programming a Simple Client}

The information given within the previous sections allow for realization of a simple client that is able to contact the XploRe server, send XploRe commands and receive and show output in the Java console.

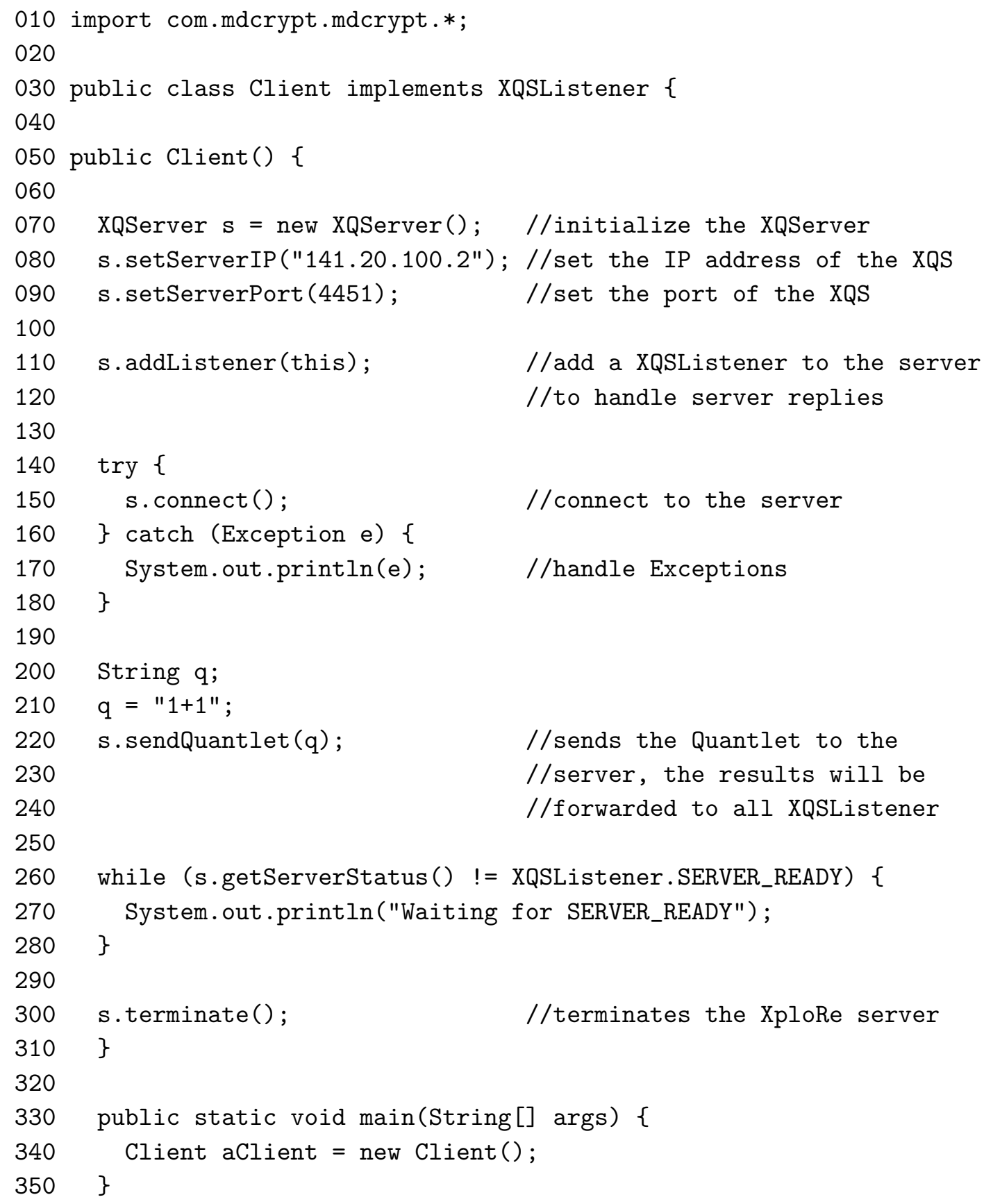


360

370

380

390

400

410

420

430

440

450

460

470

480

490

500

510

520

530

$540\}$

// receives results from server public XQSObject handleServerReply (XQSObject xqsobj) \{

if (xqsobj.getType () == XQSObject. OUTPUT) \{ XQSOutputObject xout $=$ (XQSOutputObject) xqsobj; System.out.println(xout.getText()); \}

return xqsobj;

\}

// receives the actual server status public void serverStatusChanged(int i) \{ \}

// receives information about exceptions that have occurred public void handleMdCryptException(XQSStatusMessage XqSSTM) \{ \}

Running the Java program above leads to the following output on the Java console:

Waiting for SERVER_READY

Waiting for SERVER_READY

Waiting for SERVER_READY

Waiting for SERVER_READY

Contents of _tmp

$[1] \quad$,

First of all our test client must import the MD*Crypt package (010) to be able to use its services. By implementing the XQSListener (030), the client is also forced to have the three methods handleServerReply(), serverStatusChanged() and handleMdCryptException() implemented $(380,470,510)$. This enables communication from the server back to the client. The main method (330) creates a new instance of the Client.class - actually starting the program. The first step the program completes is 
creating a new instance of MD*Crypt's XQServer.class (070). Server number (080) and port number (090) are defined in the next step. By calling the method addListener() our client is registers to receive server results (110). The XQServer.connect() method (150), "wrapped" in a try-catch statement, finally initiates the connection process to the XploRe server. A java.lang.String is filled with the XploRe command " $1+1$ ", which is supposed to be computed by the server (210). Using the sendQuantlet() method of MD*Crypt's XQServer.class finally sends the command to the server (220). The actual server status is available via the XQServer's getServerStatus() method. A loop (260), checking the status, forces the client to wait until the server is finished with its calculation. As soon as $\mathrm{MD}^{*}$ Crypt has received the server's result, it is sent to our (registered) client - the client's method handleServerReply(XQSObject xqsobj) is called (380). If the received object is of type 'OUTPUT' (390) it gets converted to an XQSOutputObject (400). The result can be accessed by using the objects getText() method, which returns the result as java.lang.String format (410). Now, that the server's status is 'SERVER_READY' again the loop (260) will be left. By calling the XQServer.terminate() method (300), the connection to the server can be canceled and the XploRe program will be terminated.

\subsection{XploRe Quantlet Client}

The XploRe Quantlet Client (XQC) represents the front end - the user interface (UI) of the XQC/XQS architecture [KL01]. The XQC is fully programmed in Java. Using a pure Java solution, the XQC does not depend on a certain computer platform. It can run on Windows and Mac platforms, as well as on Unix and Linux machines. Utilizing an application or a certified applet, the $\mathrm{XQC}$ can access the resources of the computer on which it is running. Because of Java's sandbox principle, the XQC passes underlying restrictions for accessing the local system while running as an applet. Unfortunately, these restrictions also inhibit its functionality of accessing local methods and data. Figure 3.4 shows a screen shot of the XQC running as an application.

The appearance of the XQC is based on the MS Windows XploRe version [HKM99] to ensure easy utilization and a familiar look. In comparison to the MS Windows version that only offers a CUI, the XQC combines a CUI with GUI functionality. The CUI (Character User Interface) functionality 


\subsection{XploRe Quantlet Client}

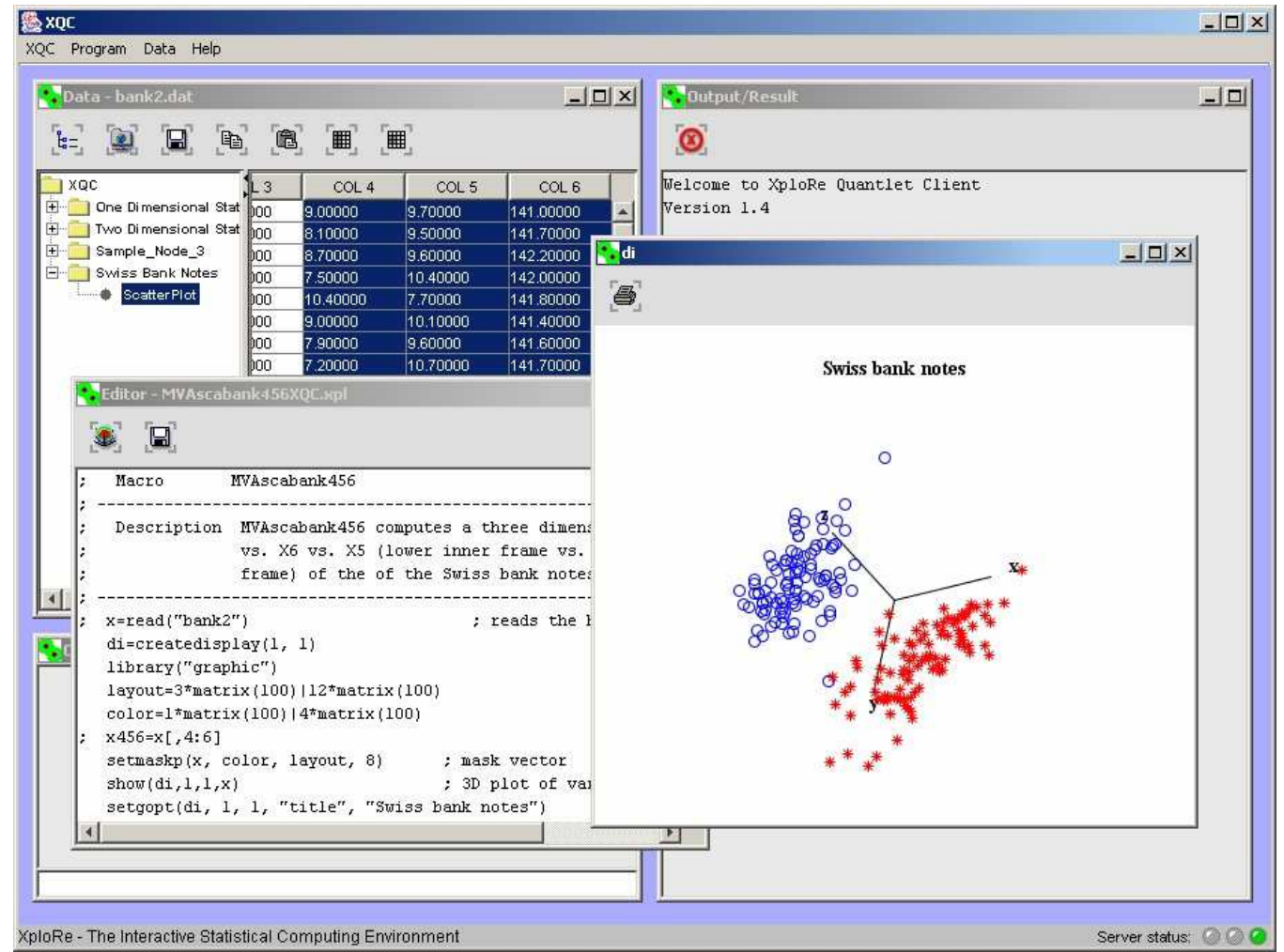

Figure 3.4: $\mathrm{XQC}$ in action

is realized by a simple input window (console) that offers direct command line access to the XploRe server and an editor window with a text area for writing and executing more than just a single line command. To make use of this functionality, the potential user needs to be familiar with the XploRe programming language.

The GUI (Graphical User Interface) functionality is realized by a combined data and method window. It enables the user to explore data with given methods without having to know the XploRe language itself. As the name implies, the data and method window consists of two parts: an excel-like table for editing the data and a tree with methods for the use with the data.

Property files allow for customizing the XQC to meet special needs and also to manage its appearance and behavior. Because of its pure Java implementation, the resulting platform independence and the ability for customization via property files, the XQC recommends itself for the integration into HTML and PDF contents (e.g. electronic books) for visualizing statistical and math- 
XploRe Quantlet Client/Server Model

ematical coherences. 


\section{Chapter 4}

\section{XQC in Detail}

The following chapter will give a detailed description of the XQC's functionality, as well as its internal structure.

\subsection{XQC in Action}

\subsubsection{Application versus Applet}

The XploRe Quantlet Client can be initiated in two different ways. The way depends on whether the XQC is supposed to run as a standalone application or as an applet embedded within an HTML page. The XQC comes packed in a single Java Archive (JAR) file, which allows easy usage. This JAR file allows for running the XQC as an application, as well as running it as an applet.

Running the $\mathrm{XQC}$ as an application does not require any programming skills. Provided that a Java Runtime Environment (JRE) is installed on the computer on which the XQC is executed, the xqc.jar will automatically be recognized as an executable JAR file that opens with the program javaw (see figure 4.1).

The XQC in this instance can be started by simply double clicking on the $x q c . j a r$ file. The configuration file $x q c . i n i$ is optional. As described in previous sections, this file contains information about server and port number. If the XQC initiates without being able to access this configuration file, it 


\begin{tabular}{|c|c|c|}
\hline Name - & Size & Type \\
\hline$\square \times q c$ quantlets & & File Folder \\
\hline $3 \times q c . i n i$ & $2 \mathrm{~KB}$ & Configuration Settings \\
\hline 当 Xqc_1.4.004.jar & $164 \mathrm{~KB}$ & Executable Jar File \\
\hline 3 xqc_language.ini & $5 \mathrm{~KB}$ & Configuration Settings \\
\hline $3 \times q c$ methodtree.ini & $2 \mathrm{~KB}$ & Configuration Settings \\
\hline
\end{tabular}

Figure 4.1: XQC - jar file

starts with a default setup, asking for a manual input of server and port information.

If the XQC is embedded in an HTML page it runs as an applet and can be initiated immediately after showing the page.

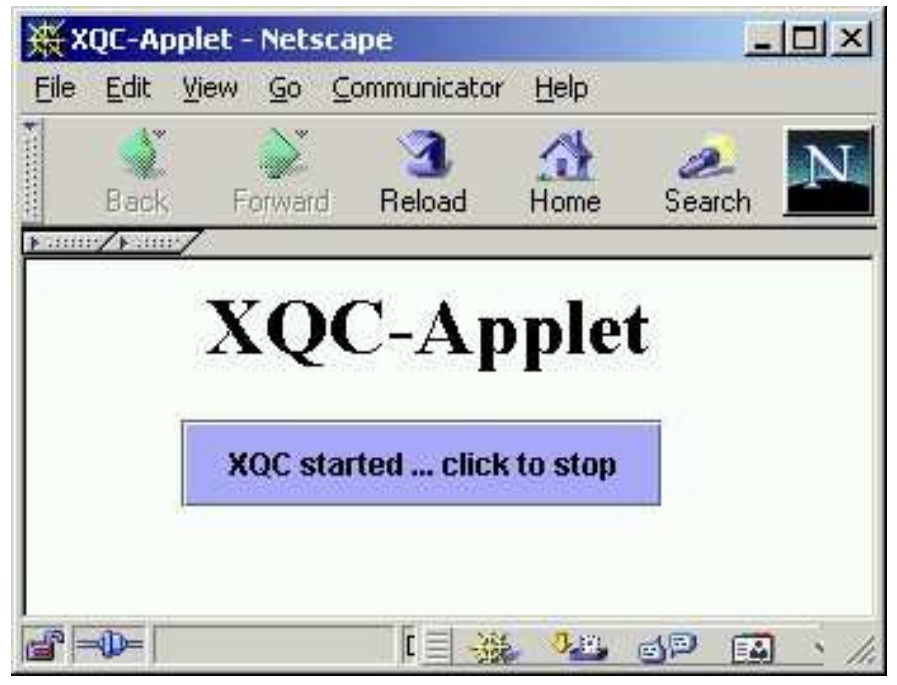

Figure 4.2: XQC started as an applet

The source code to start the XQC as an applet depends on the browser used to display the page. Figure 4.3 shows the source code for integrating the client into an HTML page that can be used with Microsoft's Internet Explorer, as well as Netscape's Navigator.

In both cases, the archive and the class that contains the main method must be stated. The XApplet.class works as the main class for starting the XQC as a Java applet. 


\subsection{XQC in Action}

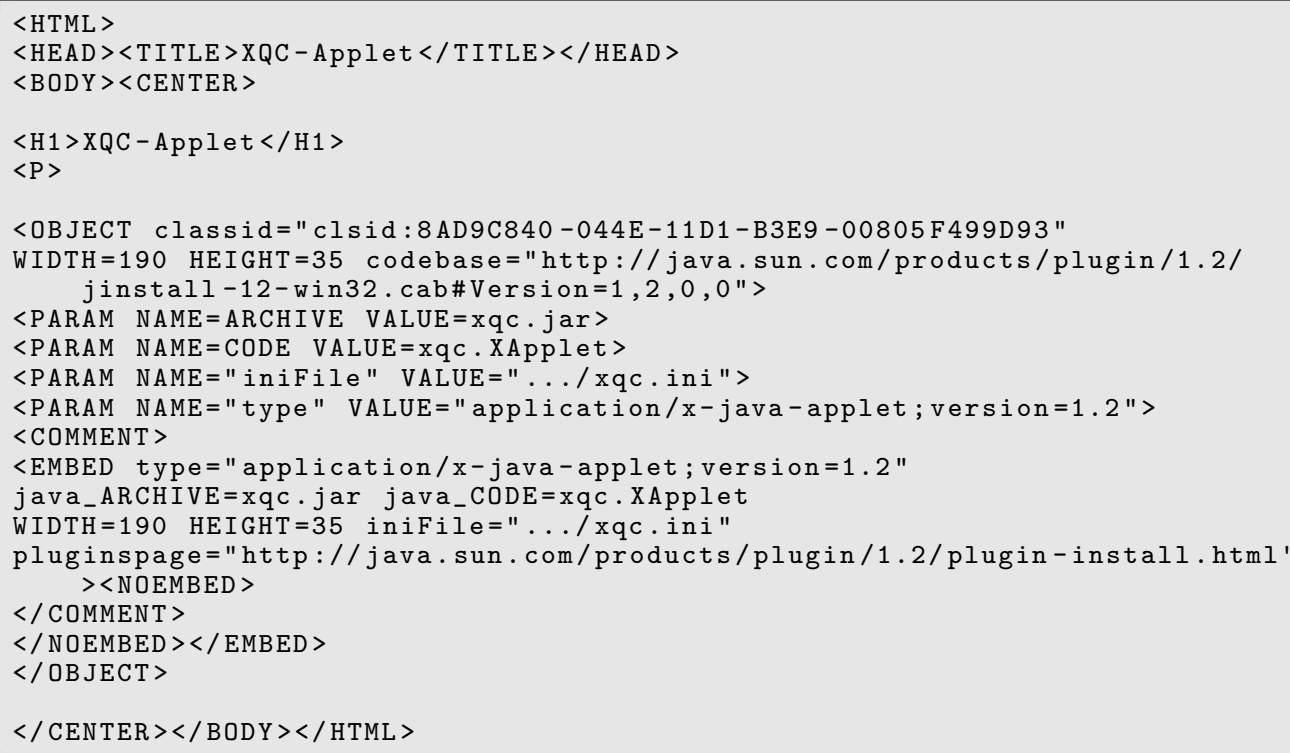

Figure 4.3: XQC - embedded in HTML

\subsubsection{Configuration}

Property files allow for configuring the XQC to meet the special needs of the user. These files can be used to manage the appearance and behavior of the XQC. As ordinary ASCII files, any text editor can alter the configuration files. Generally, the use of all information is optional. In its actual version, the XQC works with three different configuration files:

- xqc.ini,

- xqc_language.ini,

- xqc_methodtree.ini.

The file $x q c$.ini contains important information about the basic setup of the XploRe Quantlet Client, such as server and port information the client is supposed to connect to:

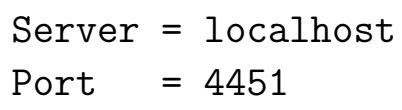


Size $=0.9$

Width $=800$

Height $=600$

It also contains information about the size of the client. This information can be maintained either relative to the actual size of the screen by using a factor or by stating its exact width and height. If this information is missing, the $\mathrm{XQC}$ begins by using its default values.

The file $x q c_{-}$language.ini allows for setting up the XQC's language. This file contains all texts used within the XQC. To localize the client, the texts have to be translated. If no language file can be found, the client starts with its default setup, showing all menus and messages in English.

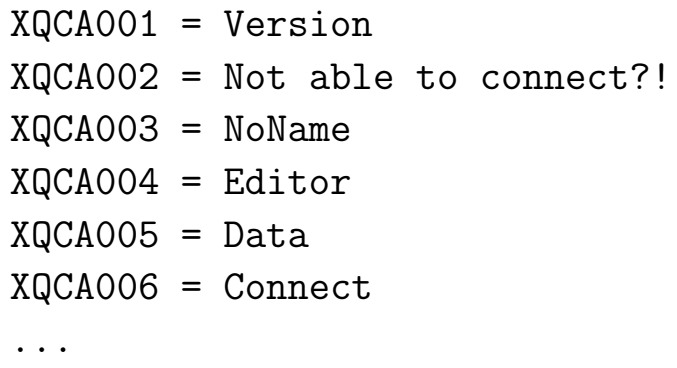

Finally, the textitxqc_methodtree.ini file contains information about the Method Tree that can be shown as part of the Method/Data Window (see 4.1.6). A detailed description of the set up of the Method Tree will be discussed in section 4.1.7.

\subsubsection{Getting Connected}

The following section describes the XQC's functionalities, assuming that the client is running as an application or a certified applet. This is the only instance when the user is able to take advantage of the entire extent of functions the XQC offers.

After starting the XQC, the client attempts to access and read information from the configuration files. If no configuration file can be found, error messages will pop up. These messages are meant to draw the users attention to the fact that the file is missing or corrupted. 
If the $\mathrm{XQC}$ is able to access the server and port information from the configuration file, it uses this information and connects to the corresponding server. If this information cannot be found, a popup appears and enables the manual input of server and port number (see figure 4.4).

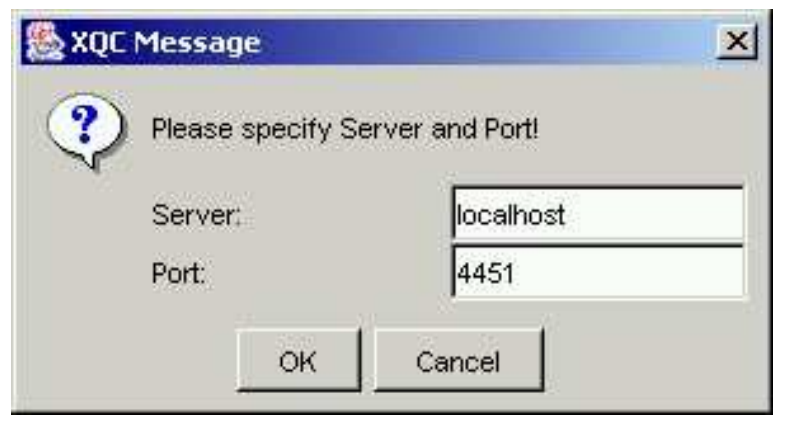

Figure 4.4: Manual input for server and port number

Figure 4.5 shows a screen shot of the XQC after it has been initiated and connected to an XploRe server. A traffic light in the lower right corner of the screen indicates the actual status of the server. A green light means the client has successfully connected to the server and the server is ready to work. If the server is busy, computing previously received XploRe code, the traffic light will be set to yellow. A red light indicates that the XQC is not connected to the server.

\subsubsection{Desktop}

If no further restrictions or features are set in the configuration file (e.g. not showing any window or starting with executing a certain XploRe Quantlet - see Section 4.1.9) the XQC should look as shown in the screen shot. It opens with the two screen components Console and Output Window. The Console (figure 4.6) allows for the sending of single-line XploRe commands to the server to be executed immediately. It also offers a history of the last 20 commands sent to the server. To repeat a command from the history, all that is required is a mouse click on the command, and it will be copied to the command line. Pressing the 'Return' key on the keyboard executes the XploRe command.

Text output coming from the XploRe server will be shown in the Output Window (figure 4.7). For our example, executed in the Console, a three- 


\section{XQC in Detail}

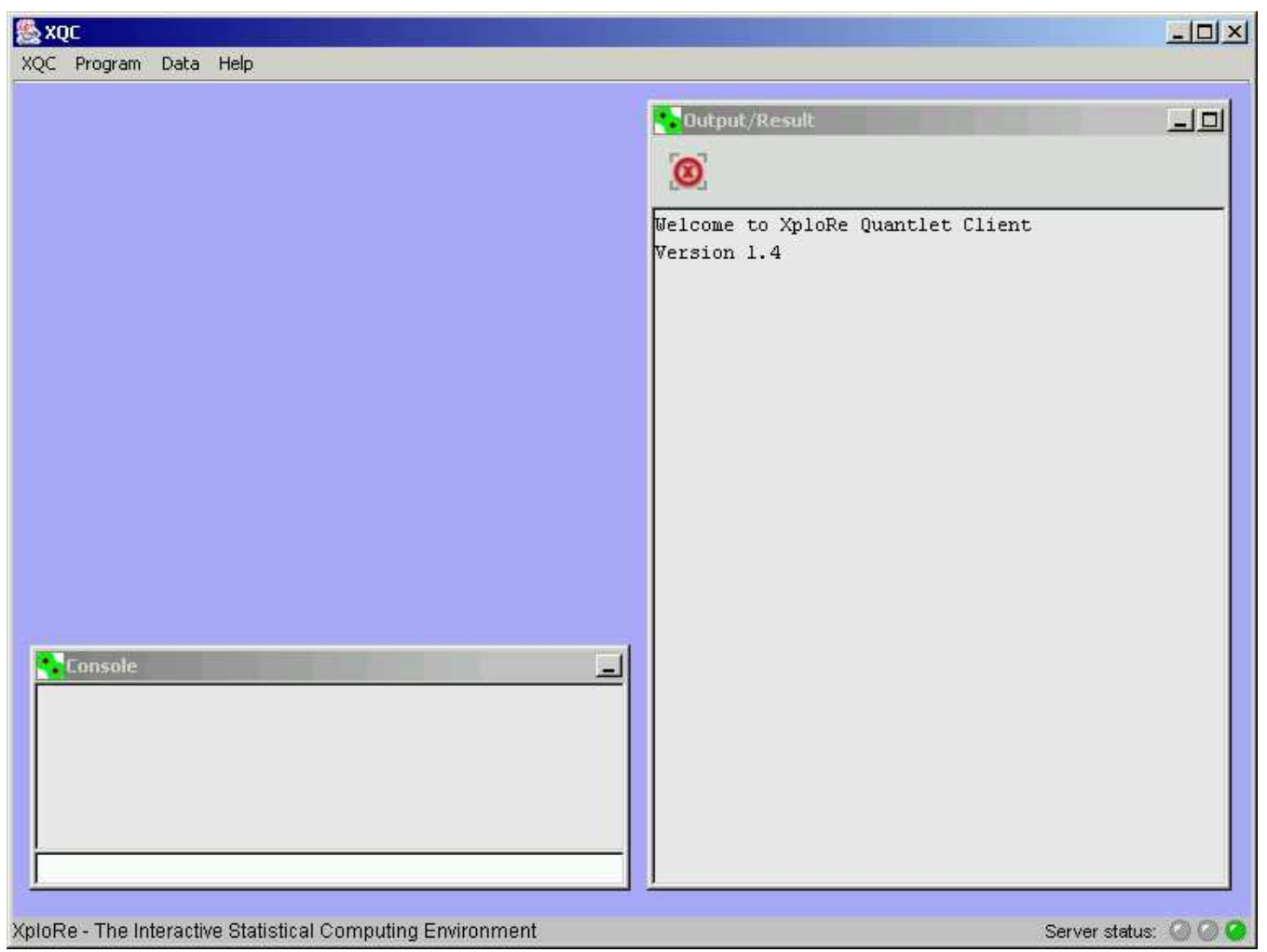

Figure 4.5: XQC connected and ready to work

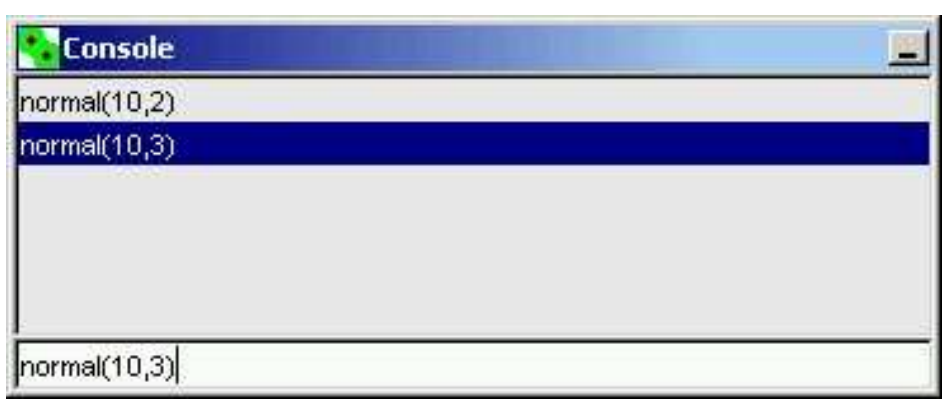

Figure 4.6: Console

dimensional, standard normal distribution with 10 rows will be created and presented as text output on the screen. Any text that is displayed can be selected and copied for use in other applications - e.g. for presentation of results within a scientific article. The [0] Icon will clear the complete 


\subsection{XQC in Action}

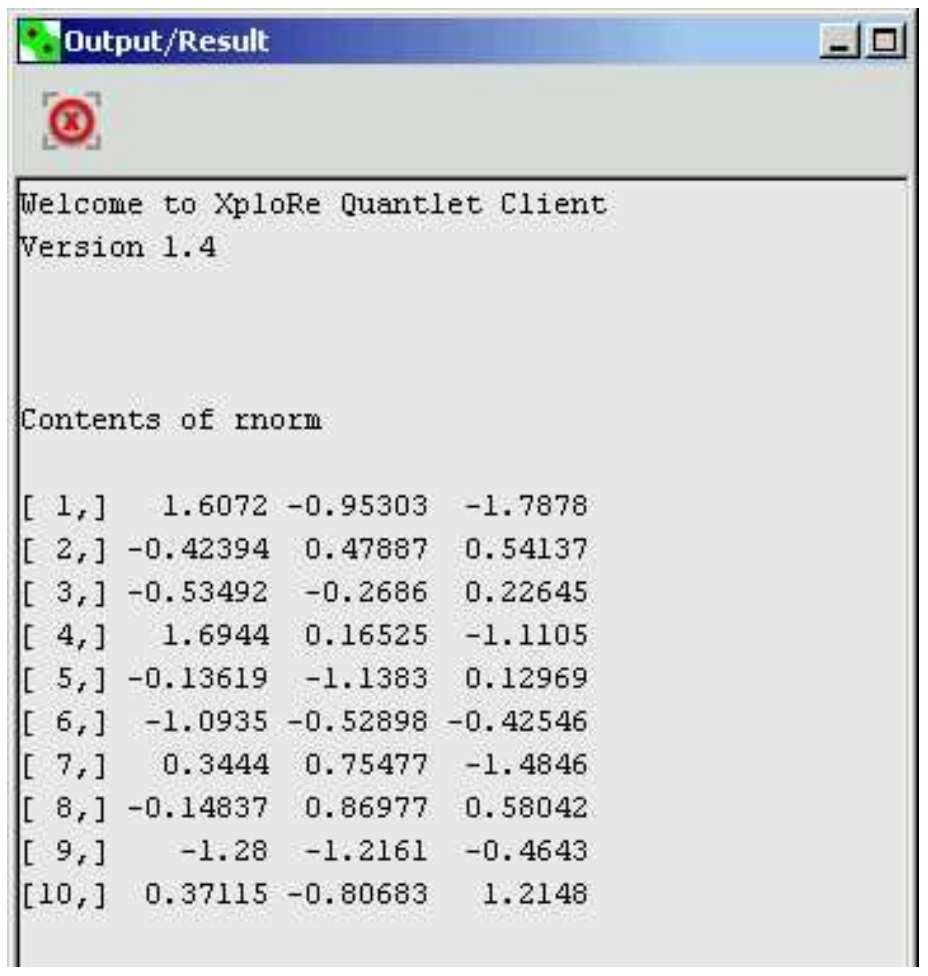

Figure 4.7: Output Window

\section{Output Window.}

The right part of the status line at the bottom of the XQC screen, per default shows the slogan "XploRe - The Interactive Computing Environment". If it has been setup in the configuration file (ShowStatusMessages = yes), at this point status messages coming from MD*Crypt protocol and the server itself, will also be visible. While running XploRe Quantlets, this line also indicates the Quantlet, the server is currently working through.

At the top of the screen the XQC offers additional functions via a menu bar. These functions are grouped into four categories:

- XQC,

- Program,

- Data,

- Help. 
The XQC menu (see figure 4.8) contains the features Connect, Disconnect, Reconnect and Quit.

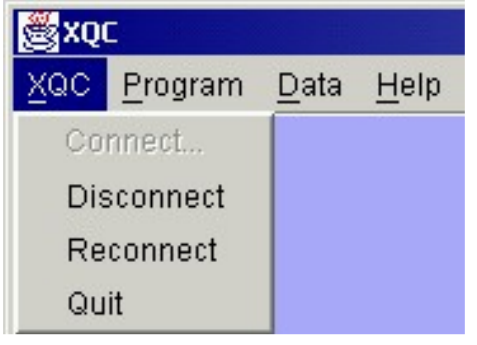

Figure 4.8: Menu 'XQC'

Depending on the actual server status, not every feature is enabled. If the client is not connected (the server status is indicated by a red traffic light), it does not make sense to disconnect or reconnect. If the client is already connected (server status equals a green light), the connect feature is disabled. All four features behave as the names imply. Disconnect disconnects client and server and closes the XploRe server process. Reconnect disconnects from the server and sets up a new connection. Quit disconnects client and server, closes the XploRe server process, and quits the XploRe Quantlet Client. The Connect feature brings up a popup as shown in figure 4.4 and enables the user to connect to a certain server and port number.

\subsubsection{XploRe Quantlet Editor}

The Program menu (see figure 4.9) contains the features New Program, Open Program (local)... and Open Program (net)....

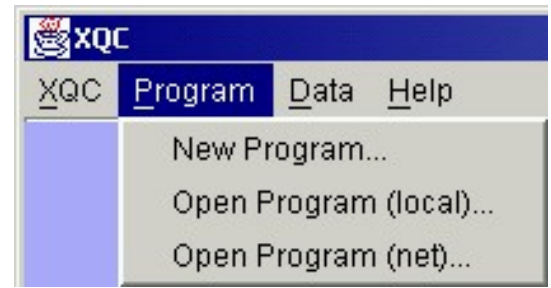

Figure 4.9: Menu 'Program' 


\subsection{XQC in Action}

New Program opens a new and empty text Editor Window. This window enables the user to write complete XploRe Quantlets.

The feature Open Program (local) offers the possibility of accessing XploRe Quantlets stored on the local hard disk drive. It is only available if the XQC is running as an application or a certified applet. Due to the Java sandbox restrictions, running the $\mathrm{XQC}$ as an unsigned applet, it is not possible to access local XploRe Quantlets.

If the user has access to the Internet the menu item Open Program (net) can be useful. This feature allows the opening of Quantlets that are stored on a remote Web server. All it needs is the filename and the URL address at which the file is located.

Figure 4.10 shows a screen shot of the Editor Window containing a simple XploRe Quantlet.

Two icons offer actions on the XploRe code:

- 4 - represents the most important feature - it allows for sending the XploRe Quantlet to the server for execution.

- [D] - saves the XploRe Quantlet to the user's local computer (this is not possible if running the $\mathrm{XQC}$ as an unsigned applet).

\begin{tabular}{l}
\hline Editor-3d.spl \\
$\begin{array}{l}\mathrm{X}=\operatorname{normal}(50,3) \\
\mathrm{Y}=\operatorname{normal}(50,3) \\
\mathrm{d}=\operatorname{createdisplay}(1,1) \\
\text { setmaskp }(\mathrm{x}, 4,3,6) \\
\text { setmaskp }(\mathrm{Y}, 2,3,4) \\
\text { show }(\mathrm{d}, 1,1, \mathrm{X}, \mathrm{Y})\end{array}$ \\
\hline
\end{tabular}

Figure 4.10: XploRe Editor Window

The Quantlet shown in figure 4.10 assigns two, three-dimensional, standard normal distributions to the variables $x$ and $y$. The generated data are for- 
matted to a certain color, shape and size using the command setmaskp. The result is finally shown in a single Display.

\subsubsection{Data Editor}

The Data menu (see figure 4.11) contains the features New Data..., Open Data (local)..., Open Data (net)..., Download DataSet from Server... and DataSets uploaded to Server.

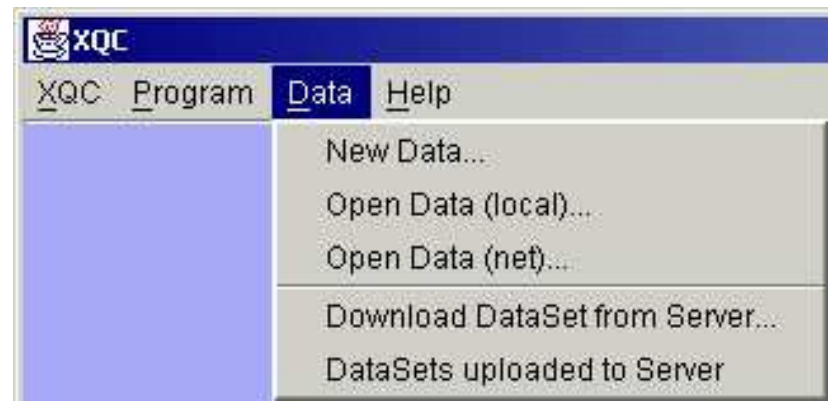

Figure 4.11: Menu 'Data'

New Data can be used to generate a new and empty Data Window. As shown later on in this section, the Data Window does not have to be just a simple data editor, but can also be configured to be used as a combined Method/Data Window. Before the Data Window opens, a pop-up window as shown in figure 4.12 appears, asking for the planned dimensions the number of rows and columns - of the new data set. The XQC requires this information to create the spreadsheet. This definition does not have to be the exact and final decision, as it is possible to add and delete rows and columns later on.

The menu item Open Data (local) enables the user to open data sets stored on the local hard disk. Again, access to local the resources of the user's computer is only possible if the XQC is running as an application or a certified applet. The file will be interpreted as a common text format file. Line breaks within the file are considered as new rows for the data set. To recognize data belonging to a certain column, the single data in one line must be separated by either using a ";" or a "tab" (separating the data by only a "space" will force the $\mathrm{XQC}$ to open the complete line in just on cell). 


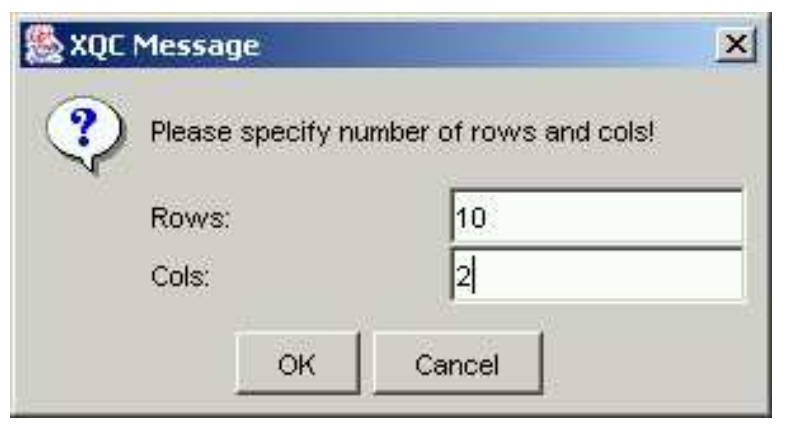

Figure 4.12: Dimension of the Data Set

Open Data (net) lets the user open a data set that is stored on a Web server by specifying the URL address.

The menu item Download DataSet from Server offers the possibility of downloading data from the server. The data will automatically be opened in a new Method/Data Window, offering all its features to the downloaded data (e.g. applying methods, saving, ... ).

The appearance of the Data Window depends on the settings in the configuration file. If a Method Tree is defined and supposed to be shown, the window shows the Method Tree on the left part and data spreadsheet on the right part of the frame. If no Method Tree has been defined, only the spreadsheet will be shown. The Method Tree will be discussed in more detail in section 4.1.7. Figure 4.13 shows a screen shot of the combined Method/Data Window.

Icons on the upper part of the Method/Data Window offer additional functionalities:

Trees are set up via configuration files.

the entire data set can be uploaded to the server by specifying a variable name.

- $[-[$ - saves the data to the user's local computer (this is not possible if running the XQC as an unsigned applet). 


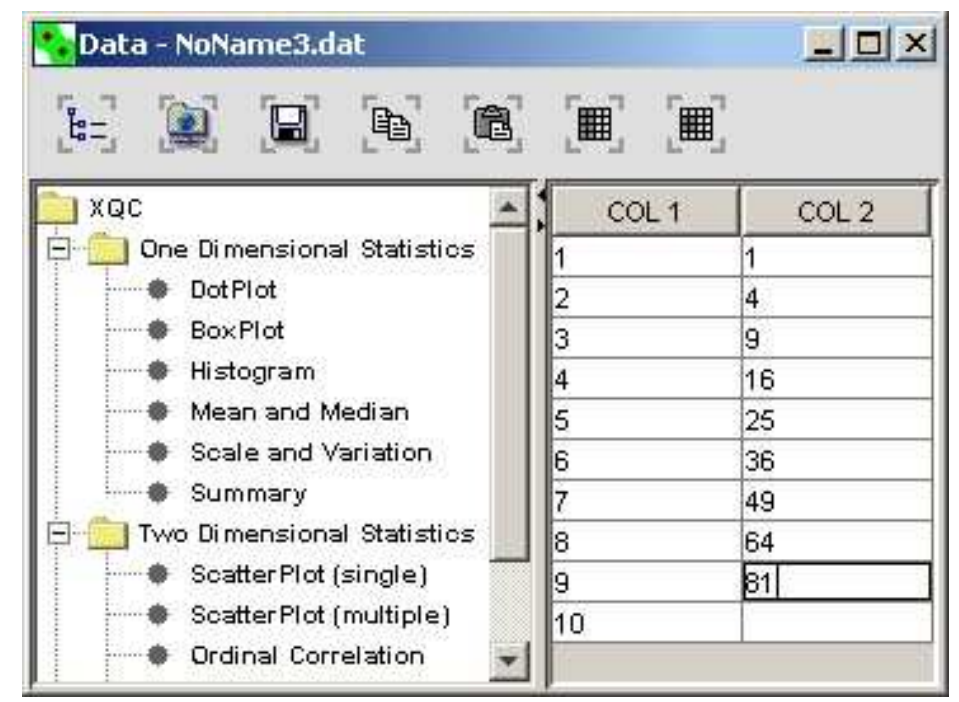

Figure 4.13: Combined Method and Data Window

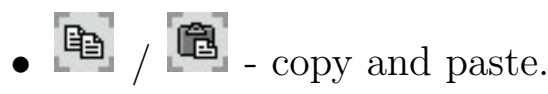

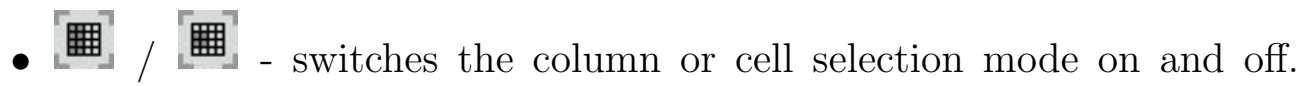
Selected columns/cells can be uploaded to the server or methods can be executed on them.

The spreadsheet of the Method/Data Window also offers a context menu containing the following items:

- Copy,

- Paste,

- No Selection Mode - switches OFF the column or cell selection mode,

- Column Selection Mode - switches ON the column selection mode,

- Cell Selection Mode - switches ON the cell selection mode,

- Set row as header line,

- Set column header, 
- Delete single row,

- Insert single row,

- Add single row,

- Delete single column,

- Add single column.

Most of the context menu items are self-explaining. However, there are two items that are worth taking a closer look at - 'Set row as header line' and 'Set column header'. The spreadsheet has the capability to specify a header for each column. This information can be used within XploRe Quantlets to name the axis within a plot, making it easier for the user to interpret graphics. A more detailed description is included in section 4.1.7. Default values for the headers are COL1, COL2, ... as shown in figure 4.14. Naming a single column can be performed using the menu item 'Set column Header'. The name has to be maintained within the pop up window that appears immediately upon choosing this menu item. It can also be used to change existing column headers. The spreadsheet also has the capability for setting column headers all at once. If the data set already contains a row with header information - either coming from manual input or as part of an opened data set - this row can be set as header using the menu item 'Set Row as Header Line'. The row with the cell that is active at that time will be cut out of the data set and pasted into the header line.

Setting the header is also possible while opening a data set. After choosing the data, a pop up asks whether or not the first row of the data set to be opened should be used as the header. Nevertheless, the context menu features described above are of course still available, enabling the user to set or change headers afterwards.

Working with the XQC's Method/Data Window does not necessitate any XploRe programming knowledge. All it requires is a pointing device like the mouse. Applying, for example, the scatter-plot-method on the two columns would require only the following three steps:

- Switch on the column selection mode,

- Mark both columns, 


\section{XQC in Detail}

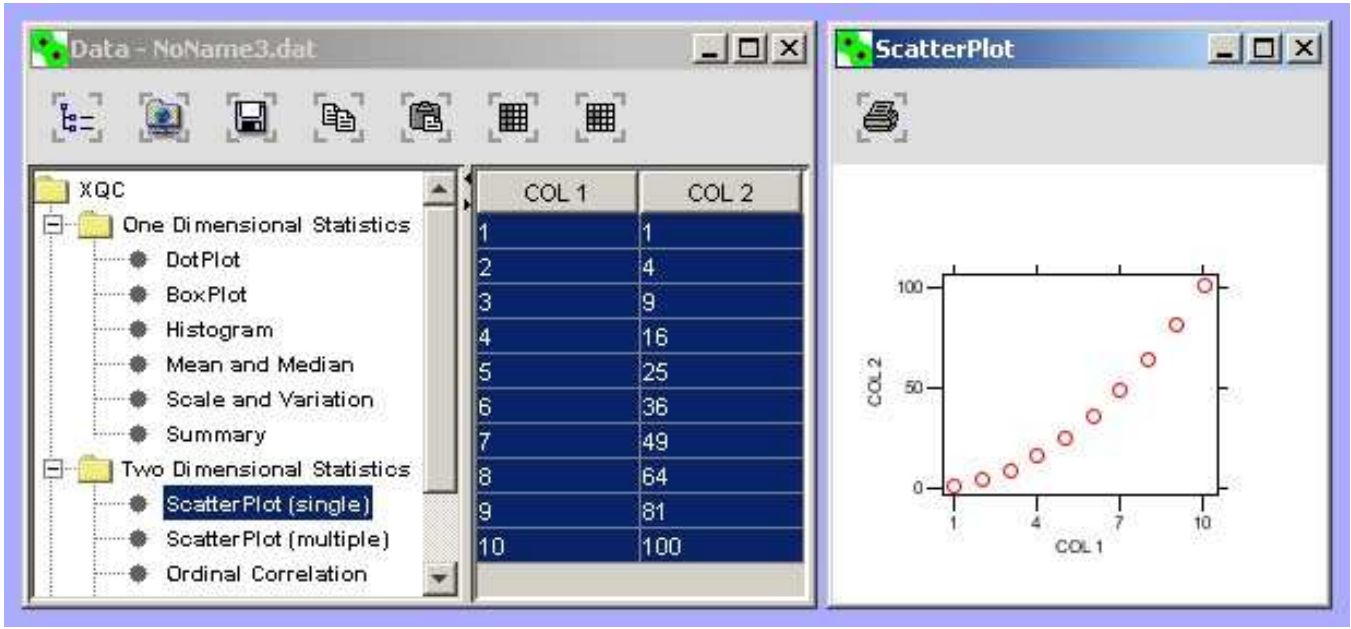

Figure 4.14: Working with the Method and Data Window

- Mouse-click on the method "Scatter Plot".

The result will be a plot as shown in figure 4.14. As stated above, the selected area can also be uploaded to the server using the icon to be analyzed for further investigation. Figure 4.15 shows the window that pops up asking for a name for storing the data at the server.

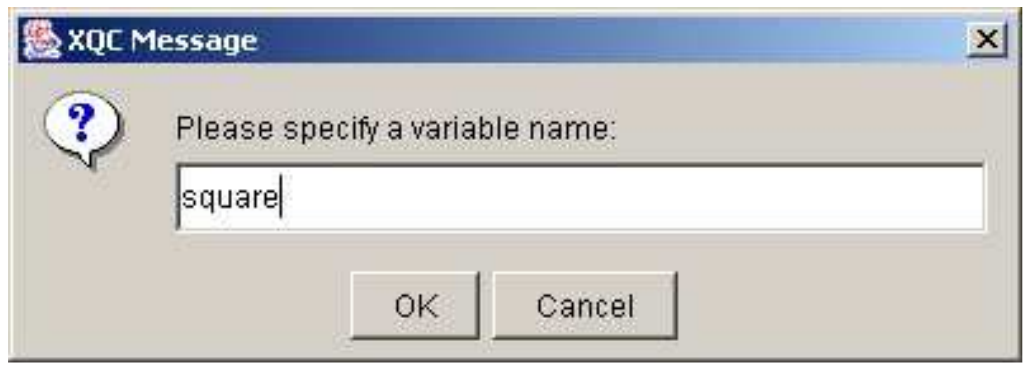

Figure 4.15: Uploading data

This new variable can be used within XploRe Quantlets, written using the Editor Window or manipulated via the Console (figure 4.16).

All actions performed via Method/Data Window and Console will be recorded by the XQC. This History can be accessed via the menu item 


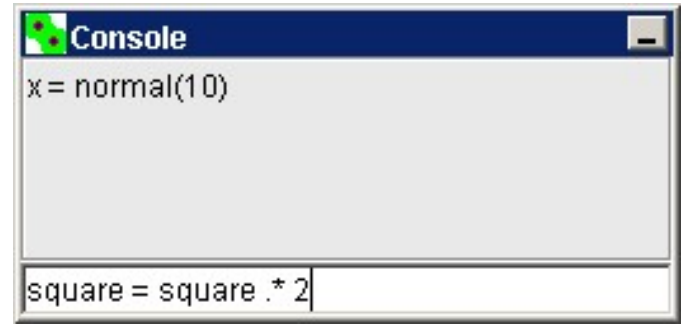

Figure 4.16: Manipulating the uploaded data

DataSets uploaded to Server. It contains a list of variable names of data known to the server. Due to performance reasons only uploaded data, as well as actions on data from the CUI component Console and the GUI component Method/Data Window are recorded. Parsing executed XploRe programs for uploaded variable information is not (yet) realized - this would slow down the speed of the program's execution. For future releases it is conceivable that the user will be able to record those data uploads and actions as well, due to adjustments made via the configuration file. Figure 4.17 shows the content of the History after having performed the actions illustrated in figures 4.13, 4.14, 4.15 and 4.16 .

The History contains two objects - variable ' $x$ ' contains a standard normal distribution generated via Console. Variable 'square' represents the content of the NoName2.dat uploaded using the Method/Data Window:

square - uploaded from data set:

NoName2 . dat

$[, 1] \sim[, 2]$

The window also contains information that the data has been changed using the Console:

'square' - Changed using the CONSOLE:

square $=$ square.$* 2$

Last but not least, it also contains the actual content of the object - in our case the doubled value of each element of the originally uploaded data set.

The icons offered by the History Window allow for adjustments to the history information according to the users needs: 


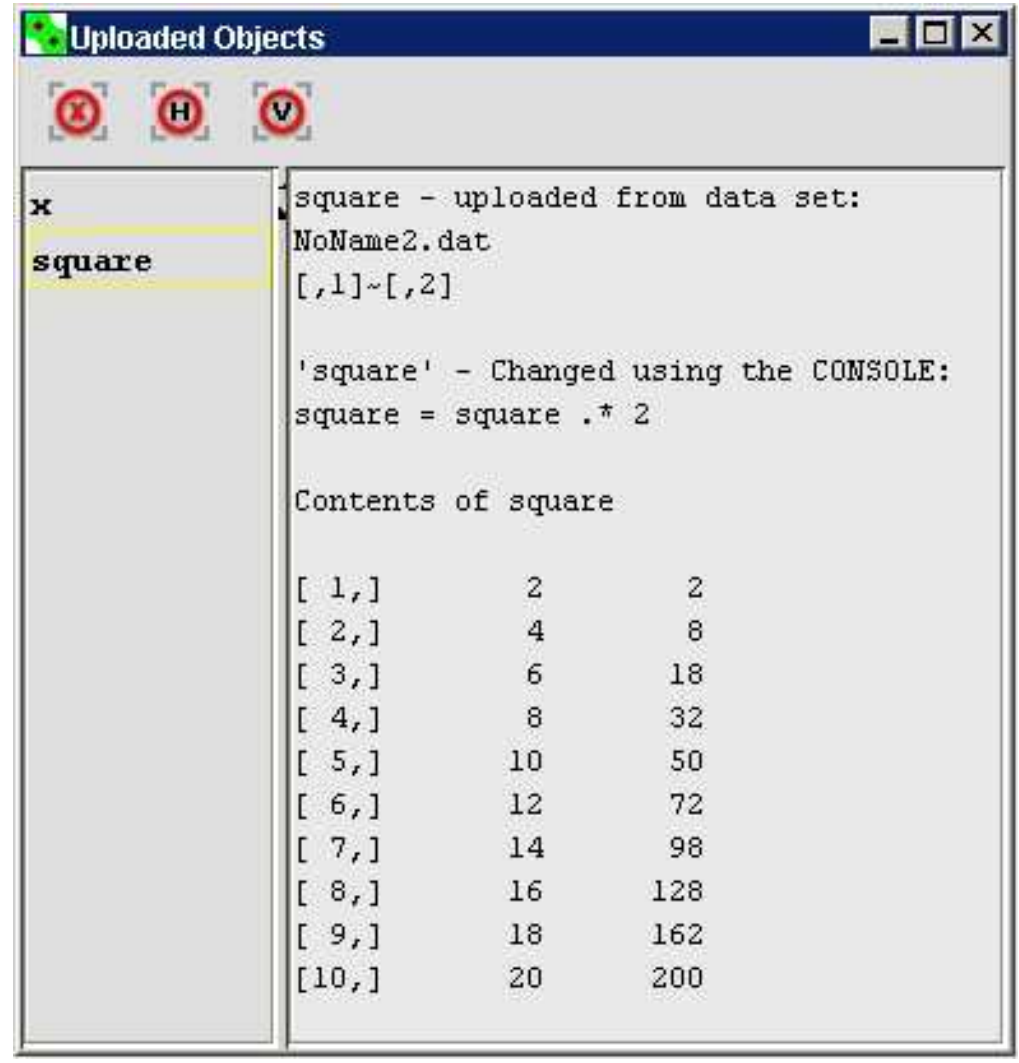

Figure 4.17: Uploaded objects

- (2) - Clears the complete list of objects.

- [H. Clears the history of the selected variable.

- [v] - Removes the selected variable from the list of objects.

\subsubsection{Method Tree}

The Method Tree represents a tool for accessing statistical methods in an easy manner. It represents a collection of executable methods that are thematically grouped. Navigation within the tree is very similar to a common file explorer (e.g. Microsoft's Windows Explorer). Analogous to the common file explorer, the Method Tree can consist of nodes and children on several 


\subsection{XQC in Action}

levels. In the actual version of the XQC (version 1.4) the number of levels is limited to four levels. Children represent statistical methods that can be used on the data. Nodes can be used to group methods.

Setting up the Method Tree does not require any Java programming skills. All it needs is the maintenance of two configuration files. This feature makes it possible to configure the XQC for different statistical or mathematical purposes, e.g. 'Basic Statistics' or 'Multivariate Statistics'. The executable methods are XploRe programs (Quantlets) that can either be stored at client side or at server side. As a defined strategy, the XQC first tries to find a method on the client's computer. Upon failure, the XQC looks for the method on the server's method pool.

Settings maintained within the $x q c . i n i$ file communicate to the XQC whether there will be a Method Tree to be shown, and from where to get the tree information. The client also needs to know where the methods are stored. The MethodPath contains this information. Path statements can be either absolute statements, or relative to the directory in which the XQC has been initiated. For relative path information, the path must start with XQCROOT. The settings in the example below tell the client to generate a Method Tree by using the file $x q c \_$methodtree.ini with the XploRe Quantlets stored in the relative subdirectory xqc_quantlets/.

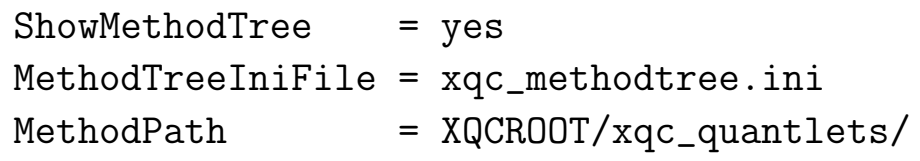

The user of XQC is not limited to the use of just one Method Tree. Instead, it is possible to integrate up to 50 Method Trees within one XploRe session, using the following additional parameters:

MethodTreeIniFile2 $=$ second_xqc_methodtree.ini

MethodTreeIniFile3 = third_xqc_methodtree.ini ..

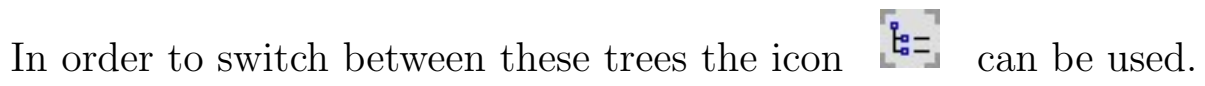

The actual Method Tree is set up in a separate configuration file that is given by the property of MethodTreeIniFile. This file contains a systematic structure of the tree (nodes and children), the method to be executed and its description to be shown within the tree frame. 


\section{XQC in Detail}

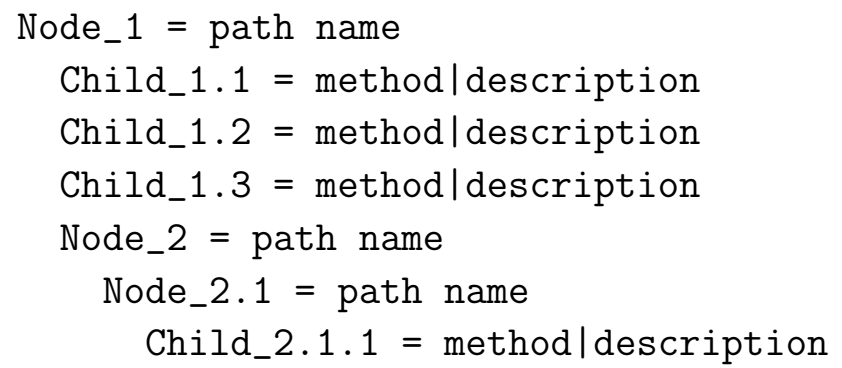

The name of the method has to be identical to the name of the XploRe program (Quantlet). The Quantlet itself has to have a procedure with the same name as the method. This procedure is called by the XQC on execution within the Method Tree.

The following example shows how to set up a simple Method Tree:

First of all, we define an XploRe Quantlet that we want to be part of the Method Tree. The aim of the Quantlet should be to generate a box plot from the selected data of the data spreadsheet. As shown in figure 4.18, the library 'graphic' is loaded to make use of XploRe's graphical features. A procedure is needed to be executed by the XQC. The name of the procedure in our case 'BoxPlot' - has to equal the name of the saved file. The procedure must further have two parameters:

- data - Used for passing the selected data to the XploRe Quantlet.

- names - Contains the names of the selected columns taken from the header of the spreadsheet.

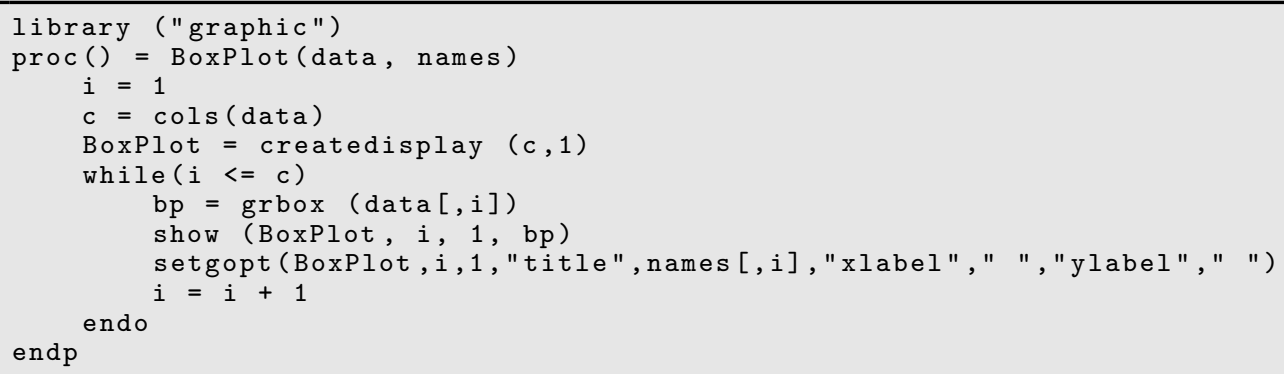

Figure 4.18: BoxPlot.xpl 


\subsection{XQC in Action}

The XploRe coding within the procedure statement is not subject to any needs or restrictions. We read the number of selected columns (4) and create a Display in which the number of plots depends on the number of selected data columns (5). Within a loop, we create the box plot by using XploRe's 'grbox' method (7) and show the result in the generated Display (8). Using XploRe's 'setgopt' statement (9) allows for setting up the title and labels. Here we can use the column header passed from the spreadsheet, setting it as the plot title.

Once we have programmed the Quantlet it needs to be integrated into a Method Tree. For this purpose we define our own configuration file - sample_tree.ini - with the following content shown in figure 4.19.

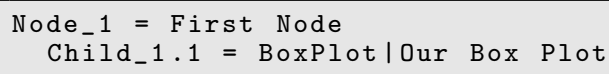

Figure 4.19: sample_tree.ini

We create a node calling it 'First Node'. Below this first node we set up our box plot - 'BoxPlot' represents the XploRe Quantlet and the procedure we have just programmed, 'Our Box Plot' represents the text we would like to be shown in the Method Tree.

Now that we have programmed the XploRe Quantlet and set up the Method Tree we still need to tell the XQC to show our Method Tree upon opening data sets.

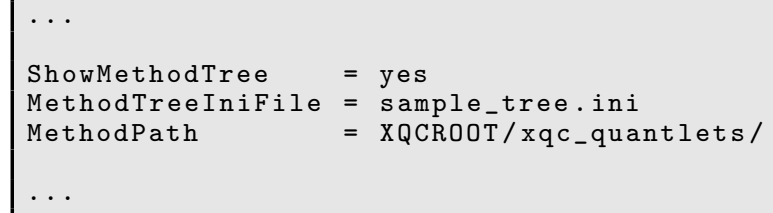

Figure 4.20: Extract of the xqc.ini

The settings as shown in figure 4.20 tell the XQC to show the Method Tree that is set up in our sample_tree.ini file and to use our XploRe Quantlet stored in a subdirectory of the XQC itself.

Now our Method Tree is ready for finally being tested. Figure 4.21 shows a screen shot of the result of the programming and the settings made above. 


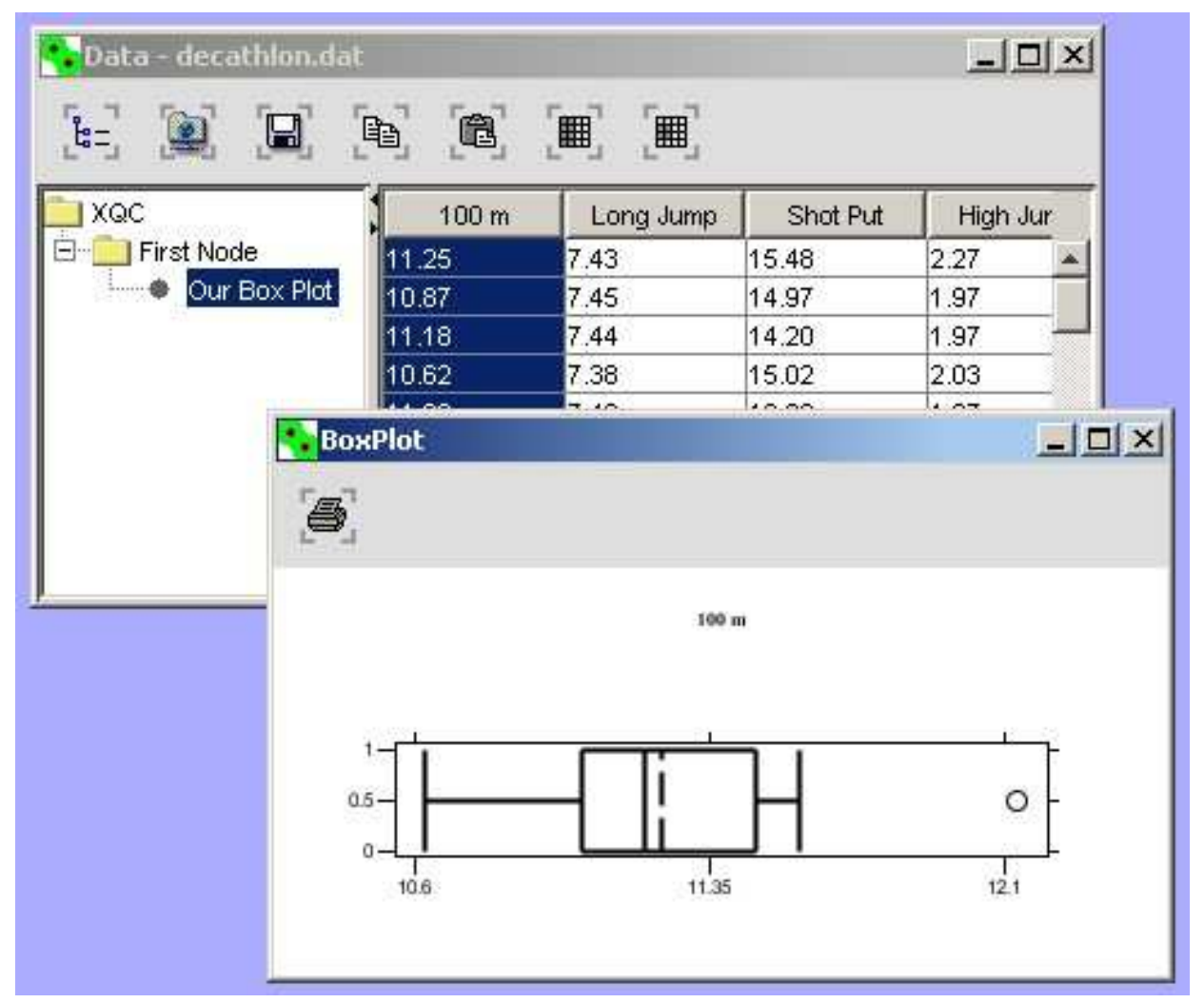

Figure 4.21: Final result of our tree example

\subsubsection{Graphical Output}

The previous sections contain some examples of graphical output shown within a Display. The XQC's Displays do not show only the graphical results received from the XploRe server. Besides the possibility to print out the graphic it offers additional features that can be helpful for investigating data - especially for three-dimensional plots. Those features can be accessed via the Display's context menu. Figure 4.22 shows the three-dimensional scatter plot for three characteristics (lower inner frame vs. diagonal vs. upper inner frame) of a collection of 200 Swiss bank notes - containing 100 notes that are actually counterfeits (this example is part of the electronic book 'Multivariate Statistical Analysis' which can be downloaded at http://www.iXploRe.de/ebooks/).

The scatter plot shows genuine and counterfeit bank notes using a different look and color. As a default setting, the X-Y-dimension will be shown. 


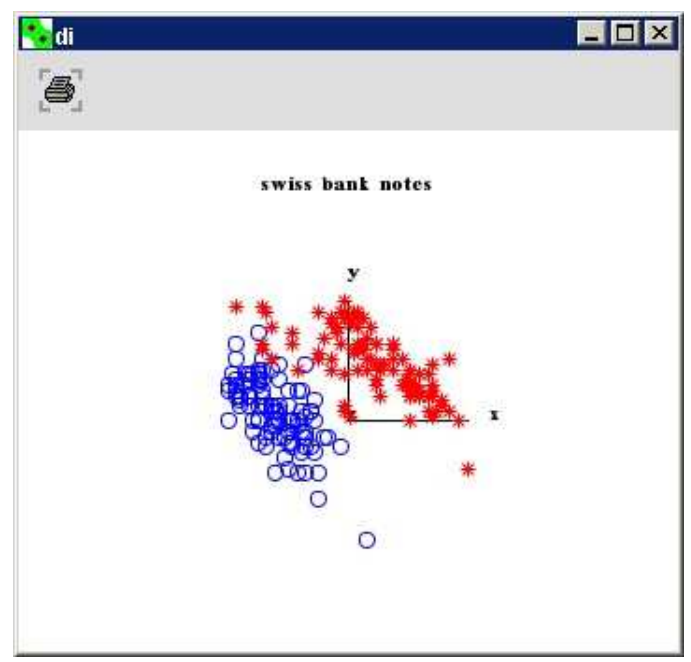

Figure 4.22: Scatter plot for characteristics of Swiss bank notes

The scatter plot implies that there exist two clusters - genuine and counterfeits, although some bank notes are overlapping when only the characteristics 'lower inner frame' and 'diagonal' are considered. For a more detailed inspection, three-dimensional plots can be rotated by using a pointing device such as a mouse (with the left mouse-button pressed) or by using the keyboard's arrow-keys. Figure 4.23 shows the same plot as before - it has just been rotated by some degrees. Now, also considering the characteristic 'upper inner frame', the clusters of the data are even easier to identify. However, there still is one data point (represented by a blue circle) that lies among the data of the other cluster (represented by red stars). For further research, it would be helpful to know which data point it is. Of course the user could compare the characteristics of all data points to find the one. Here the XQC's Display offers a feature to show the point's coordinates. This feature can be accessed via the Display's context menu. 'Showing coordinates' is not the only option. The user could also switch between the three dimensions 'Show $\mathrm{X}^{\sim} \mathrm{Y}^{\prime}$, 'Show $\mathrm{X}^{\sim} \mathrm{Z}$ ' and 'Show $\mathrm{Y}^{\sim} \mathrm{Z}$ '.

After the 'Showing coordinates' option has been chosen, all that is required to get the information is to point the mouse arrow on a certain data point. Figure 4.23 shows the details ' $1 / 70[8.0,11.2,139.6]$ ' for the data point that seems to be part of the 'wrong' cluster. '1/70' implies that we deal with data point number 70 of the first data set printed within the Display. The information 'first' is important since there could be more than just one data 


\section{XQC in Detail}

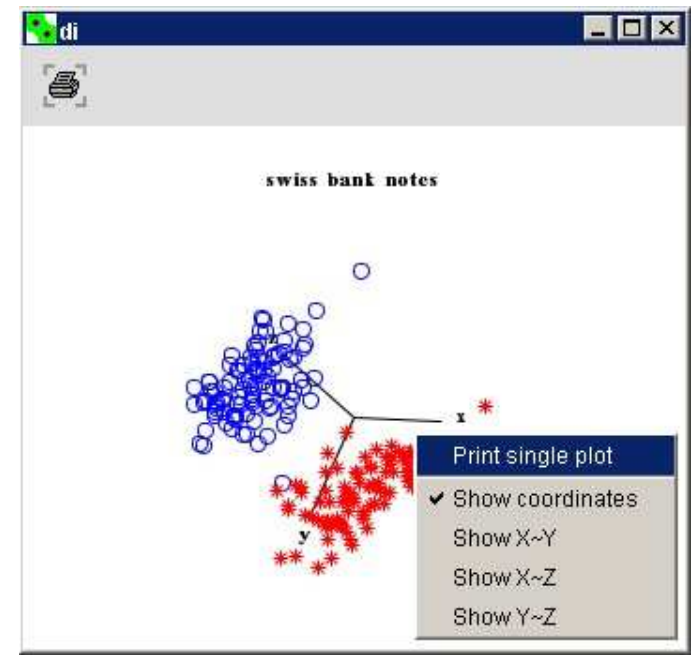

Figure 4.23: Rotating scatter plot showing the context menu

set shown in the Display. The numbers within the brackets '[...]' are the actual characteristics of that data point.

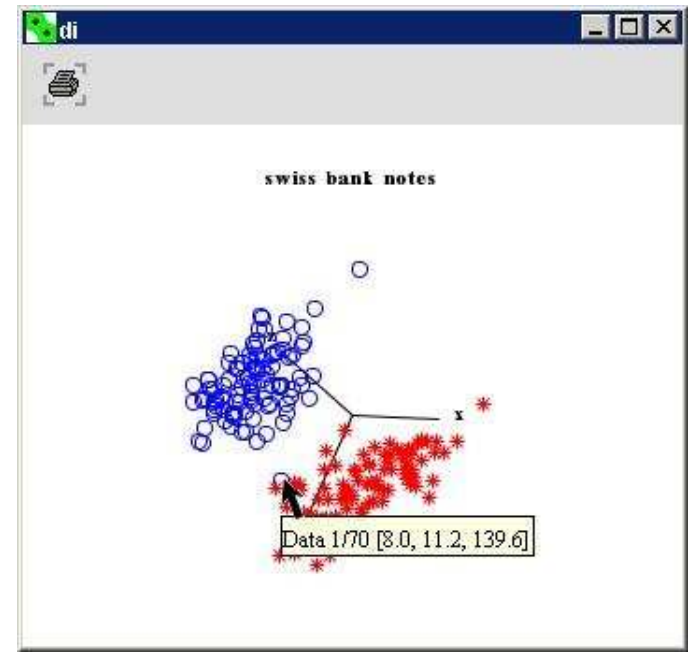

Figure 4.24: Showing the coordinates of a data point 


\subsection{XQC in Action}

\subsubsection{Special Settings}

The configuration file $x q c$.ini offers some more options than just storing server and client size information. Different property statements can be used to influence the appearance and behavior of the XploRe Quantlet Client.

$\begin{array}{ll}\text { ExecuteCommands } & =\text { normal }(10,2) \\ \text { ExecuteProgram } & =\text { file: } / / / \mathrm{C}: / \ldots / \text { normal } . x p l\end{array}$

As the name implies, the statement 'ExecuteCommands' allows for stating a single XploRe command. This XploRe code will be executed immediately after the client has been started. The XQC behaves in the same manner as if the command would have been executed via its Console. The upper example command 'normal $(10,2)$ ' results in the generation of a two-dimensional, standard normal distribution that will be shown in the Output Window. The statement 'ExecuteProgram' goes one step further, allowing for an automatic execution of complete XploRe Quantlets. Path statements are possible

- as absolute paths - locally (file:///..) or URL address (http://...)

- relative to the directory in which the XQC has been started (XQCROOT/...).

The 'OpenInEditor' feature works in a similar fashion. It opens the XploRe Quantlet with the designated option, but without executing the coding. The execution portion is up to the user. This feature allows for inspecting XploRe Quantlets, changing or adjusting and finally executing them. The same settings as described above are applied to path statements. The example below opens a the Quantlet regression.xpl which is stored on the local drive on which the $\mathrm{XQC}$ is running.

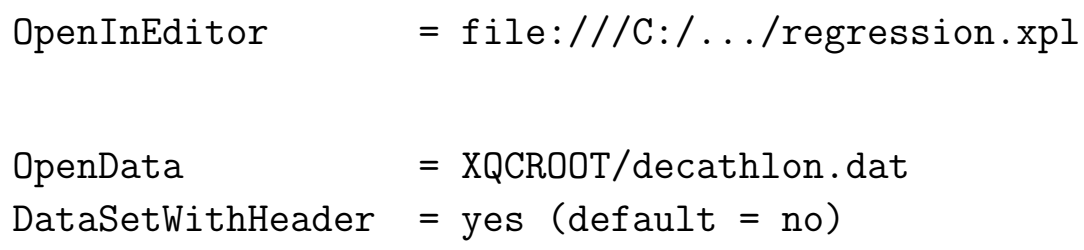




\section{XQC in Detail}

The XploRe Quantlet Client can not only be started by opening an XploRe Quantlet, but also by opening a certain data set in the Method/Data Window. For this purpose, the feature 'OpenData' can be used. As described in the previous sections, the Method/Data Window offers the possibility of maintaining column header for graphical output. If the data set contains a header, the feature 'DataSetWithHeader = yes' automatically sets the first line of the data set as the header line. Default setting for this feature is 'DataSetWithHeader $=$ no'. In this case, the first line will not be used as the header line.

If Output Window and Console are not needed - the following configuration settings can be used to switch them off:

ShowOutputWindow $=$ no $($ default $=$ yes $)$
ShowCommandWindow $=$ no $($ default $=$ yes $)$

All the settings described above belong to what we call the "Golden Solution". Using these settings, it is possible to influence each component of the XploRe Quantlet Client. This allows for embedding of the XQC into multimedia contents for different purposes, controlling its behavior via the configuration settings. It can be started by executing a certain Quantlet stated in the file without displaying Console or Output Window. In this case the XQC behaves like a Java applet programmed for a particular task. It can also be started by opening a data set with certain predefined and customized methods to execute the methods on the data set.

Starting the XQC by performing one of the specific actions described above causes the client to run in a mode with limited functionalities. In this mode, it is only possible to work with the initiated XploRe Quantlet, edit and execute the opened Quantlet, or execute predefined methods on the opened data set. Per default it is not possible to open new programs or data. In most of these cases the XQC is supposed to work only for the predefined purposes. This limitation is inherent in the "Golden Solution" definition in order to not confuse the user with any functional overhead. To avoid this limitation, the "Golden Solution" mode can be switched off by using the following statement:

GoldenSolution $\quad=$ no $($ default $=$ yes $)$ 
An additional statements offers the opportunity to get a closer look behind the scenes of the working XQC. Switching on the 'ShowStatusMessages' displays messages coming from the server and from MD*Crypt. For example, those messages can permit the user to track the server's way through the XploRe Quantlets, showing the procedure the server is currently processing. The drawback of this feature is a slightly decreased performance of the XploRe server because of the additional 'workload'.

ShowStatusMessages $=$ yes $($ default $=$ no $)$

The possibility of configuring the XploRe Quantlet Client for special purposes, as well as its platform independence, are features that recommend themselves for the integration into HTML and PDF contents for visualizing statistical and mathematical coherences - [RMZ00, Kli01]. Sections 6 and 7 contain examples that illustrate how the XQC can be used for different results.

\subsubsection{Getting Help}

The XQC's Help menu contains the two menu items Online Help and About ... The Online Help menu item offers direct access to the XploRe APSS - Auto Pilot Support System (http://www.xplorestat.de/help/_Xpl_Start.html), provided that an Internet access is available.

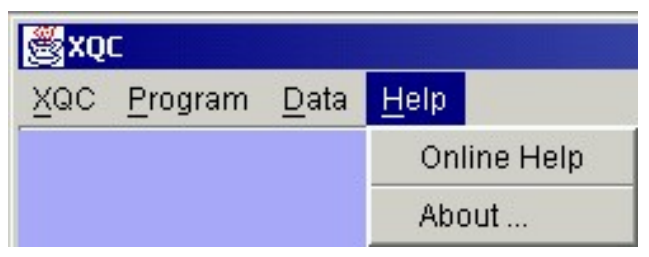

Figure 4.25: Menu 'Help'

XploRe's APSS is a complex help tool, realized in HTML. It can be used with common browsers. The APSS works as an XploRe tutorial, as well as containing a collection of XploRe's function groups and libraries with its according Quantlets. Each available Quantlet is presented with a comprehensive description, including an example. 


\section{XQC in Detail}

The second menu item - About ... - opens a window that contains information about the client/server architecture currently being used, as well as information about the Java Runtime Environment (JRE) in which the XQC is running.

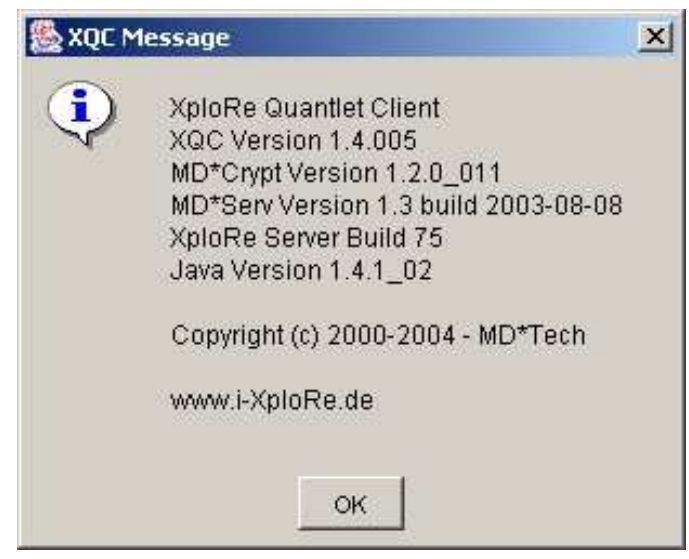

Figure 4.26: Version information

\subsection{Programming Structure of the XQC}

The general programming structure of the XploRe Quantlet Client reflects the components and functions described in the previous section. Due to the characteristics of the Java programming language, all components of the XQC are encapsulated in their own class or object respectively. This objectoriented characteristic of the Java language offers the possibility of reusing certain objects in other projects. The XQC's plot classes - that will also be discussed in this section - are just one example.

Figure 4.27 shows the general structure of the XQC and its main components. Each square implies a single Java class. Besides the classes shown in this figure, there also exist, what we would call, "helper classes", which are used by other classes within the process. Examples of these "helper classes" are: a dialog class for message and error dialogs and classes to handle access to local files and Internet files (data and methods). The Appendix (B) contains the Java source code of selected XQC classes. 


\subsection{Programming Structure of the XQC}

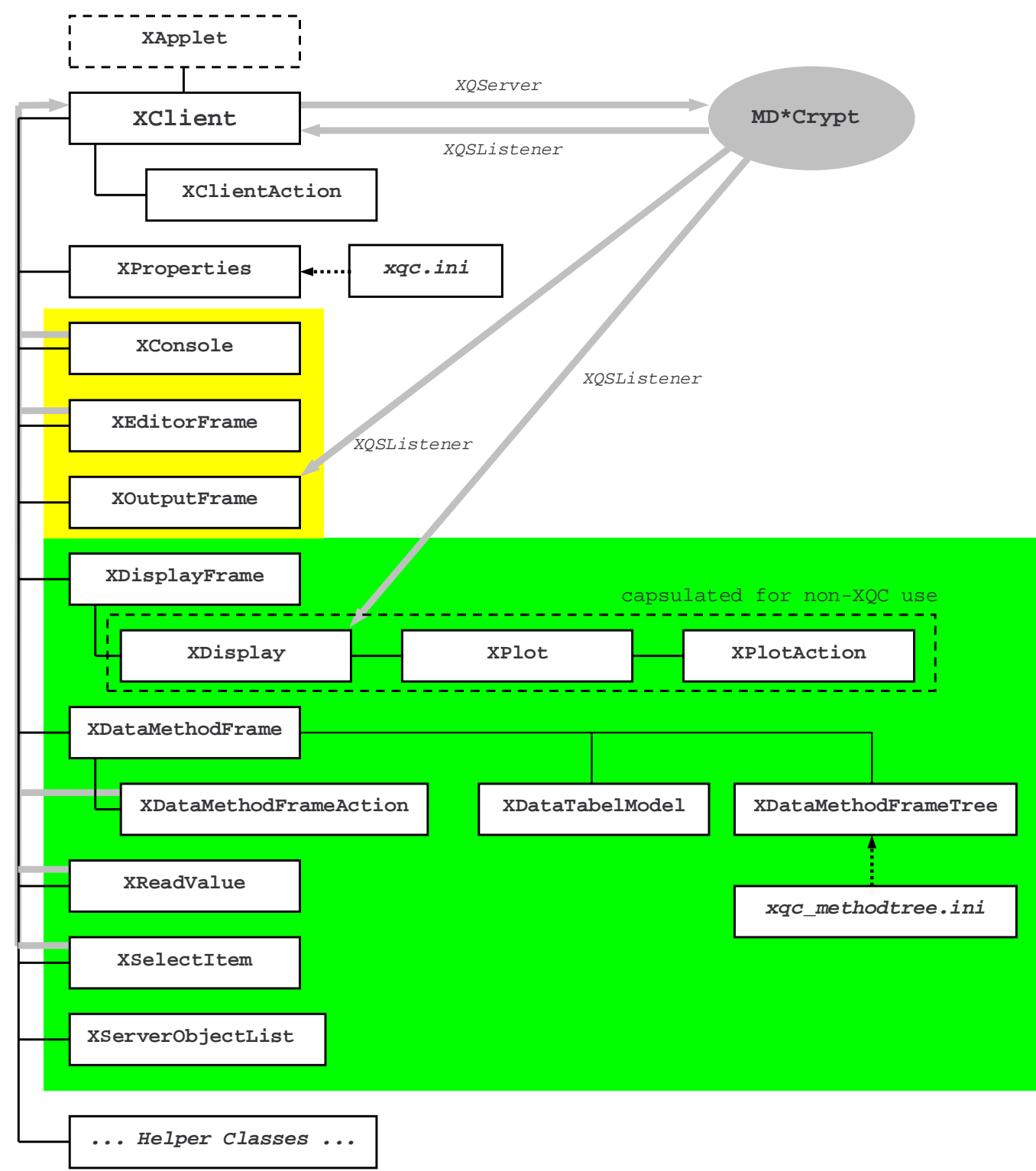

Figure 4.27: XQC structure

\subsubsection{XClient - The Starting Point}

The XClient.class (see appendix B.1) works as the "Starting Point" or "Main Class" of the XQC. Its main-method creates a new instance of the XClient. The Desktop Window with its menu bar represents this object visually. The first activity the XClient-object performs is creating an instance of the 


\section{XQC in Detail}

XProperties.class. This class extends Java's property class to offer access to the settings and information in the configuration files xqc_language.ini and xqc.ini. For a detailed description, see section 4.1.2. Using the server and port information from the configuration file $x q c$.ini, the XQC attempts to establish a TCP/IP connection to the XploRe Quantlet Server. For this purpose, it uses MD*Crypt's services by creating an instance of the XQServer.class (see 3.3.2). This XQServer object works as the one and only interface for sending information from client components to the XploRe Quantlet Server server. Every component of the XQC's structure that needs to talk to the server must use the sendQuantlet() method of this XQServer object. In order to receive results sent back from the server, the XClient.class also implements MD*Crypt's XQSListener and registers itself at the XQServer. According to the Listener concept, it must therefore also define the following three methods:

- serverStatusChanged(int status),

- handleMdCryptException(XQSStatusMessage xqsstm),

- handleServerReply(XQSObject xqsobj).

The serverStatusChanged() information is used to show the actual server status - indicated by the traffic light on the lower right part of the XQC's desktop. The handleServerReply() method handles incoming server results. Depending on the type of the received server object, the XClient.class triggers different events:

- Result type 'CREATE_DISPLAY' - creates a new Display by implementing a new instance of the XDisplayFrame.class. This Display works as a container for the plots that contain the graphic.

- Result type 'SELECT_ITEM' - creates a new instance of the XSelectItem.class. This class contains the logic to realize XploRe's 'select item' functionality.

- Result type 'READ_VALUE' - creates a new instance of the XReadValue.class. This class contains the logic to realize XploRe's 'read value' functionality. 
- Result type 'SET_SIZE'/'AXIS_OFF'/'AXIS_ON' - these result types contain information for graphical presentations - size of a Display, whether axis within plots should be printed or not. The information is kept in the static variables of the XClient.class.

- Result type 'STATUS' - status messages coming from XQS and MD*Crypt are shown on the lower left part of the XQC's desktop.

The complete handling of events performed within the XClient.class is realized in a separate class - the XClientAction.class. This class handles all events triggered from the XQC's menu bar. It connects and disconnects with the server and creates new editor or data windows. It also keeps track of the number of opened windows to ensure that newly opened windows (including displays) are not completely overlapping each other, but are arranged in a cascading style.

If the XQC is used within an HTML page the XApplet.class works as the entry point for the client. This class extends Java's JApplet.class. It defines the necessary methods init(), start(), stop() and destroy(). The aim of the $X$ Applet.class is to start the $\mathrm{XQC}$ as a Java applet - creating a new instance of the XClient.class.

\subsubsection{User Interfaces}

How can a user interface be defined? An user interface can be seen as a component that allows for interaction with another computer or, as in our case, with a server respectively. In general, there exist two different types of user interfaces that can be distinguished - a Character User Interface (CUI) and a Graphical User Interface (GUI).

A Character User Interface (CUI) presents information to the user as text. It requires the user to type commands (known as command lines) to run programs. Unix and MS DOS are examples of CUIs.

A Graphical User Interface (GUI) on the other hand is an interface consisting of graphical elements such as windows, icons and, as with the XQC, of trees with underlying functionalities. The user can select and activate these options by utilizing pointing devices such as a mouse. Since the user does not have to type in certain code in order to use options, functions or to start programs, the GUI is much easier to use. 
Most of today's computer applications interact with the user via Graphical User Interface, although the Character User Interface is still a widely used tool for experienced computer users, especially for those working in the Unix world.

\subsubsection{Character User Interface - CUI}

The XQC's CUI is primarily meant for experienced XploRe users. It requires the user to be familiar with the XploRe programming language.

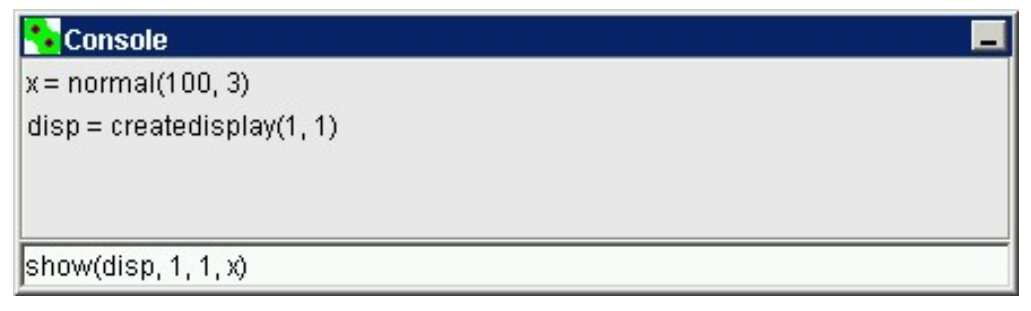

Figure 4.28: Console

\section{CONSOLE}

The XQC's component Console (see appendix B.5) represents one part of the architecture's Character User Interface (CUI). It consists of a command line window that allows the entering of single-line commands which can be sent to the server for direct processing. The Console itself does not have an XQSListener implemented and consequently does not receive any results coming from the XploRe Quantlet server. A history of the last 20 commands sent to the server helps to keep an overview of what has been sent to the server. They can easily be selected and executed again. Taking a closer look at Java code - the Console is realized within the XConsole.class extending a javax.swing. JnternalFrame. The frame itself holds two components - a javax.swing.JTextField that represents the single command line and javax.swing. JList that holds the list of executed commands.

To be able to react to user actions, the XConsole.class implements the java.awt.event.KeyListener, as well as the javax.swing.event.ListSelectionListener. The XQServer object that has 
been created within the XClient.class is used for sending a command directly to the XploRe server.

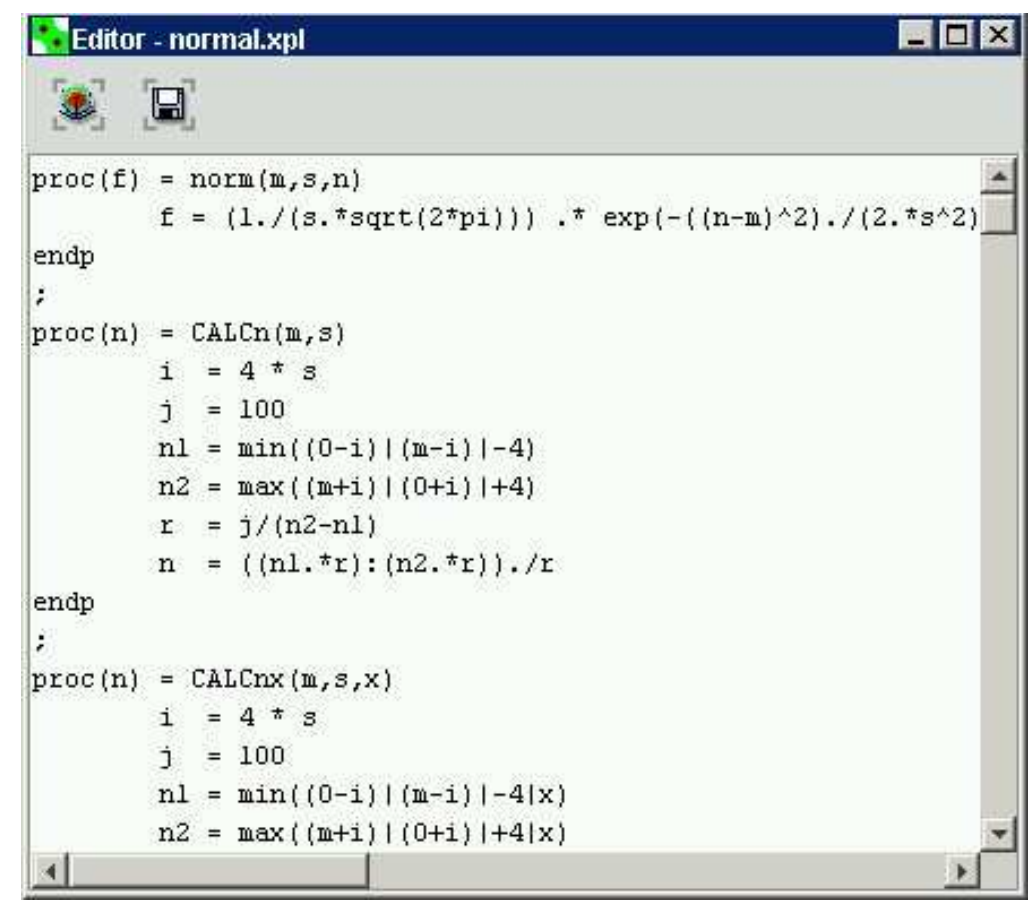

Figure 4.29: Editor Window

\section{EDITOR}

The Editor (see appendix B.6) represents an extension of the single-line Console. This component is also part of the Character User Interface (CUI). It allows for writing and editing complete XploRe programs (Quantlets). Unlike the Console, this XploRe code will not be sent to and executed by the server after typing in a single line. Instead, the execution is triggered for the complete XploRe Quantlet by clicking the according 'Execute' icon. Realization of this component is still kept very simple. The XEditorFrame.class extends a javax.swing.JInternalFrame to be placed on the client's desktop. A javax.swing.JTextArea within this frame enables the editing. The XEditorFrame.class has one constructor, with two parameters:

protected XEditorFrame(XClient client, int editorNo) \{ 
$\cdots$

\}

The parameter 'client' is needed in order to send the programmed XploRe Quantlet to the server using the implemented XQServer object. The parameter 'editorNo' tells the Editor the number of already opened frames, to generate a default file name, and to determine its position on the client's desktop.

The Console, as well as the Editor does not receive any results coming from the server. Depending on its type, the result is either shown as text within the Output Window or presented graphically in a Display (part of the GUI).

As mentioned above, the main task of the Output Window is the presentation of text output coming from the server. Extending a javax.swing.JInternalFrame the XOutputFrame.class implements MD*Crypt's XQSListener. This enables the Output Window to become aware of results from the XploRe Quantlet server. Only received content from type 'OUTPUT' is processed - shown in the window's javax.swing. JTextArea without any type of conversion or filtering.

\subsubsection{Graphical User Interface - GUI}

GUI is primarily thought of as being for users that are not familiar with the statistical programming language XploRe and/or users that want to apply existing statistical methods on their own data sets. The basic feature of a GUI is a surface that can be controlled intuitively. A simple mouse-click can trigger functions of the underlying program without the need for the user to type a single line of code.

\section{DESKTOP WINDOW}

The XQC's graphical user interface consists of several components as shown in figure 4.27. Immediately after starting the client a Desktop Window appears on the screen. Its menu bar offers the following options: to connect and disconnect with an XploRe Quantlet server; open and save programs and/or data sets; to download data objects from the server. Due to the 
sandbox concept of the Java programming language, it must be distinguished between running the XQC as an application, a certified or pure applet. Pure applets are limited in their functionality. They are not allowed to access local files or to use the copy and paste functionality of the underlying operating system. To be able to access the full functionality of the XQC, it should be started as an application or a certified applet.

\section{METHOD/DATA WINDOW}

The most important GUI component for communication with the XploRe server is the combined Method/Data Window. As shown in figure 4.30 this component is realized by the classes:

- XDataMethodFrame.class,

- XDataMethodAction.class,

- XDataTableModel.class,

- XDataMethodFrameTree.class.

Extending Java's javax.swing. JInternalFrame the XDataMethodFrame.class (see appendix B.8) works as the main class of the Method/Data Window. It generates the frame that holds the other components such as the menu bar, the spreadsheet and the Method Tree. The XDataMethodFrame.class has three different constructors used within three different situations.

- public XDataMethodFrame(XClient client, int dataNo)

This constructor is called if the user wishes to open a new frame not containing any data. In this case, a dialog forces the user to enter the number of rows and columns that need to be created. As in the Editor Window, the parameter 'client' is needed to enable the Method/Data Window to communicate with the XploRe Quantlet server. Whereas, the parameter 'dataNo' contains the information of actually open windows. Using the information pertaining to number of rows and columns, this constructor also creates an empty array of strings to be shown in the spreadsheet. 


\section{XQC in Detail}

- public XDataMethodFrame(XClient client, int rows, int cols, int dataNo)

As compared to the previous constructor this one contains two more parameters - the number of rows and columns. It is called if the user wants to download a data set from the server, triggered via the XQC's menu bar. The client receives a XQCDoubleMatrix from the server (see also section 3.3.2). This object contains the number of rows and columns as parameters used to create an empty spreadsheet. The data itself is passed to the spreadsheet using the setValueat(...) method of the XDataTableModel.class.

- public XDataMethodFrame(XClient client, String dataString, int dataNo, boolean withColHeader)

Lastly, this constructor also obtains the data that is supposed to be shown in the spreadsheet. Due to the fact that this constructor is used for data sets opened from the local computer or from an Internet address, the data is passed to the XDataTableModel.class in a java.lang.String format. To show the data in the spreadsheet, the 'dataString' has to be divided into its single values. This process is carried out by the stringToTable() method of the XDataMethodFrame.class. The result is an array of strings, ready to be shown in the spreadsheet. The parameter 'withColHeader' contains information as to whether or not the first row of the data set should be used as a column header.

All three constructors refer to the same initialization process of the XDataMethodFrame.class. Within this process, the single components of the Method/Data Window - menu bar, table and method tree - are created.

The Table is realized using Java's swing component JTable. Two parameters - a table model and a column model - pass set-up information to the table. The XDataTableModel.class (see appendix B.10) defines the table model by implementing Java's TableModel interface. This package offers methods for accessing the data within the table. Defining a separate column model enables the use of additional features such as adding and removing columns from the table.

Besides JTable, the component JTree is one of the elementary components of Java's swing package. The JTree component is used to realize the Method 


\subsection{Programming Structure of the XQC}

\begin{tabular}{|c|c|c|c|c|}
\hline \multicolumn{3}{|l|}{-Data - decathlon.dat } & \multicolumn{2}{|c|}{- - 므 $x$} \\
\hline$r_{0}={ }^{7}$ & 嘈电 & & & \\
\hline \multirow{3}{*}{$\begin{array}{l}\text { XQC } \\
\text { One Dimensional Statistios } \\
\text { Two Dimensional Statistios }\end{array}$} & $100 \mathrm{~m}$ & Long Jump & Shot Put & \multirow[b]{2}{*}{$2-$} \\
\hline & 11.25 & 7.43 & 15.48 & \\
\hline & 10.87 & 7.45 & 14.97 & 1 \\
\hline ScatterPlot (single) & 11.18 & 7.44 & 14.20 & 1 \\
\hline - ScatterPlot (multiple) & 10.62 & 7.38 & 15.02 & 2 \\
\hline - Ordinal Correlation & 11.02 & 7.43 & 12.92 & 1 \\
\hline - Discrete Correlation & 10.83 & 7.72 & 13.58 & 2 \\
\hline Regression & 11.18 & 7.05 & 14.12 & 2 \\
\hline$\square$ Sample_Node_3 & 11.05 & 6.95 & 15.34 & $2-$ \\
\hline - method_3.1 & 11.15 & 7.12 & 14.52 & 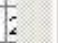 \\
\hline$\square$ Node_3.2 & 11.23 & 7.28 & 15.25 & 1 \\
\hline - method_3.2.1 & 10.94 & 7.45 & 15.34 & 1 \\
\hline$\bigoplus$ Node_3.2.2 & 11.18 & 7.34 & 14.48 & 1 \\
\hline - method_3.2.2.1 & 11.02 & 7.29 & 12.92 & $i$ \\
\hline method_3.2.2.2 & 10.99 & 7.37 & 13.61 & 1 \\
\hline$\pm \quad$ Node_3.2.3 & 11.03 & 7.45 & 14.20 & 1 \\
\hline & 11.09 & 7.08 & 14.51 & $2+$ \\
\hline & 4 & & & \\
\hline
\end{tabular}

Figure 4.30: Method and Data Window

Tree functionality of the XQC. The basis for building a tree is a data model that contains the information regarding nodes and children, which are part of the tree. This information is provided by the XDataMethodFrameTree.class (see appendix B.11). The name of the file that contains information regarding the set up of the Method Tree is passed to this class. The XDataMethodFrameTree.class itself extends a Java's Properties class. This is necessary in order to read the tree information from the configuration file. In its current version (1.4.005) the XQC can handle up to four levels (including node and child levels). As shown in section 4.1.7, the definition of the Method Tree looks as follows:

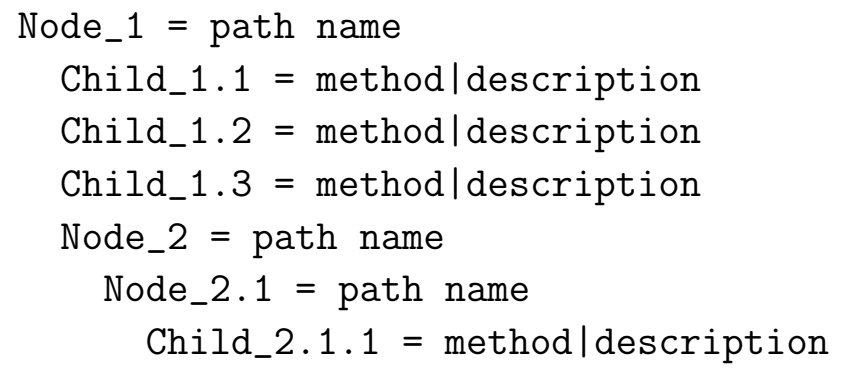




\section{XQC in Detail}

The statements Node_1, Child_1.1,...Node_2.1 and Child_2.1.1 work as the property keys for the Method Tree. To build up the tree in the correct order, the XDataMethodFrameTree.class has to read the information in the proper sequence. Figure 4.31 shows the parts of the Java code that build up the tree.

Based on the potential four levels, the basis for realization are four interlaced 'do ... while' loops. Variables 'nodeName' and 'childName', both arrays of java.util.String, hold the properties of the single node and child statements. Nodes and children are realized using Java's javax.swing.tree.DefaultMutableTreeNode. Node information must only be shown within the Method Tree - it does not need any additional functionality. Children on the other hand, need to be processed in a different way. As with nodes, they are part of the tree, but a child also contains information about an XploRe Quantlet that can be executed. This information must be divided into the name of the Quantlet and its description. The method childMethodDescription() parses the 'childName', splits the string at the "" and returns an array that contains the name of the executable Quantlet and the description to be shown within the tree. A very important role is carried out by the variable 'noOfChildren'. As a two dimensional array of strings, it holds the information regarding the relationship between the executable Quantlet and the child's description or the child's path within the tree respectively. If the user selects a specific method, javax.swing.event.TreeSelectionEvent is triggered. This event contains information regarding the selected child, including the complete path. Using the variable 'noOfChildren', this information leads to the XploRe Quantlet that will be executed.

All events triggered within the Method/Data Window are handled by the XDataMethodAction.class (see appendix B.9). This class implements all action listeners (e.g. ActionListener, TreeSelectionListener, TableModelListener) that are necessary to react to any user action within the Method/Data Window. These actions include method selections as well as selecting, adding or deleting columns, or uploading data. One of the most important methods implemented within this class is handleMethod(). The aim of this method is to execute an XploRe Quantlet on selected data. In section 4.1.7 we have created a simple tree example. What happens after selecting the method "Our Box Plot"? Following the extraction of the data column, we would like to investigate, the XploRe code that finally executes the Quantlet is generated. This code consists of: 


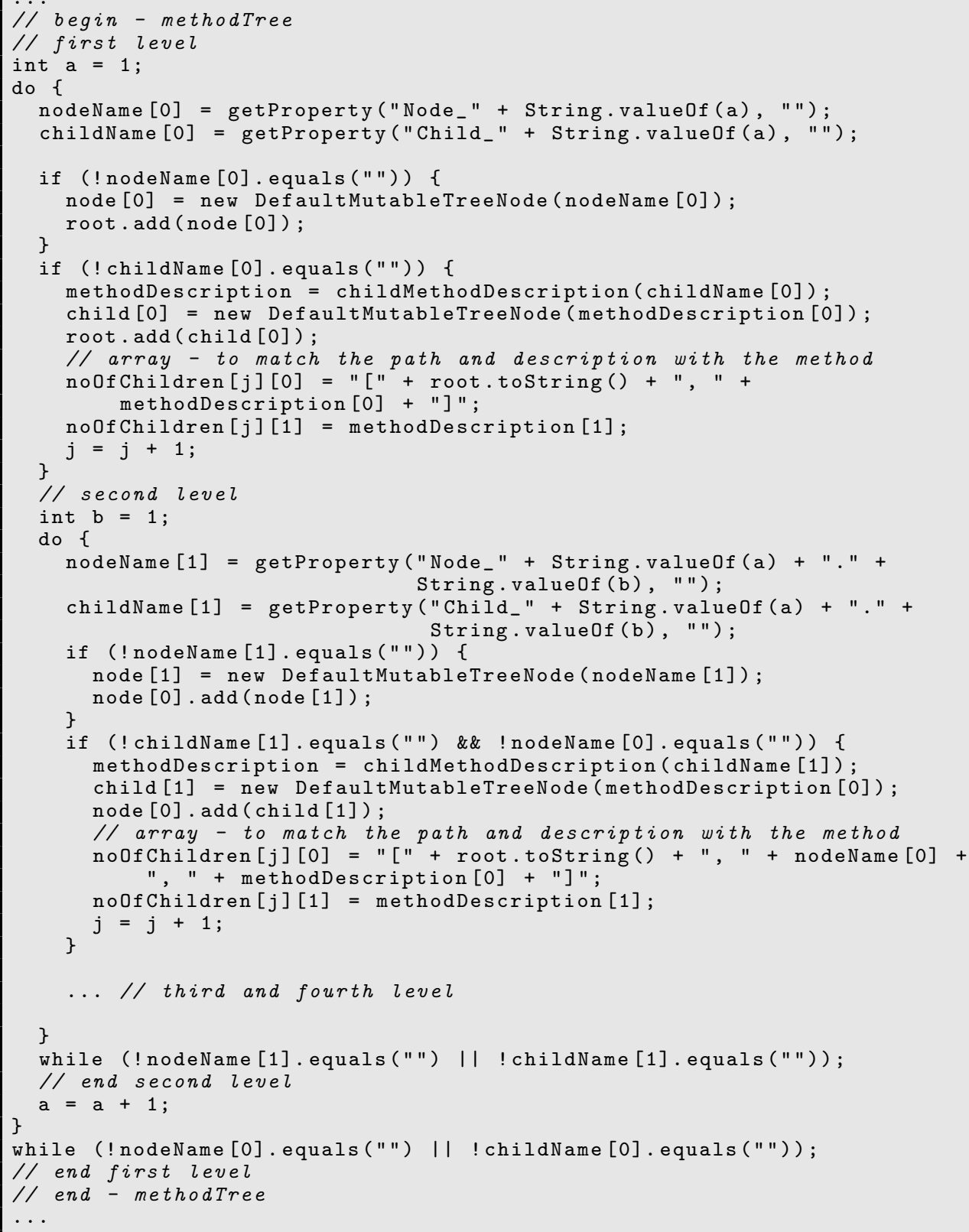

Figure 4.31: Building the Method Tree

- The complete content of the XploRe Quantlet that stands behind "Our Box Plot" - the XploRe code shown in figure 4.18. 


\section{XQC in Detail}

- The XploRe procedure call, containing the data and column names as parameters - BoxPlot (data $[, 1]$, "100 m")

The first part initiates the execution of the XploRe code at the server. Now, because the server knows the contained procedure it can be called with our selected data. Result is an XploRe Display as shown in figure 4.21.

\section{DISPLAY}

Output within the GUI is realized throughout the Plot Classes. These classes are responsible for presenting server results graphically. In order to receive results from the server, the Plot Classes have implemented MD*Crypt's XQSListener interface. The Plot Classes consist of four different main components:

- XDisplayFrame.class,

- XDisplay.class,

- XSetGOpt.class,

- XPlot.class,

- Axis.class,

- HAxis.class,

- VAxis.class,

- XPlotAction.class.

The phrase "Form Follows Function" absolutely fits those four main components. The XDisplayFrame.class utilizes a desktop frame that works as a container for the actual Display. The Display is realized by the XDisplay.class. Every XploRe Display contains one or more plots. Each plot is realized by a separate instance of the XPlot.class. All actions carried out within a Display are handled by the XPlotAction.class. Besides those four main components, a few other classes implement additional logic used within the plot classes. The XSetGOpt.class handles XploRe's "setgopt" command. 
This class allows for setting titles and labels of plot, or the size of a Display. Three axis classes (Axis.class, HAxis.class, VAxis) are responsible for drawing horizontal and vertical axis within two dimensional plots.

How do the four main components work together?

If the XQC's main class - XClient.class - receives server results of type 'CREATE_DISPLAY', it creates a new instance of the XDisplayFrame.class (see appendix B.12). It also passes over information about Display ID and number of plots that are to be part of the Display. The aim of the XDisplayFrame.class is to build a window that will be shown on the XQC's desktop, and to call a new instance of the XDisplay.class. Both classes could actually have been realized within one single class. The reason for using two classes is an encapsulation of the 'pure' Plot Classes (XDisplay.class, XPlot.class, XPlotAction.class). This encapsulation makes it possible to use its functionalities outside the XQC, by integrating the Plot Classes within other (3rd party) clients that would like to access the XploRe server [Mor04]. An example of how the 'plot classes' can be integrated into other clients that want to access the XploRe server is also part of the appendix (see appendix C).

The XDisplay.class (see appendix B.13) extends a common javax.swing.JPanel which allows for integration into any other common Java component (e.g. swing.javax.JFrame). It offers two different constructors:

- public XDisplay(int id, int rows, int cols, XDisplayFrame frame)

This constructor is used if a Display has to be created within the XQC. Besides information about 'id', 'rows' and 'cols', the parent component is also passed to the XDisplay.class. This enables easy reaction by XploRe features for setting title or Display size.

- public XDisplay(int id, int rows, int cols)

If the plot classes are used as part of a 3rd party client the type of the parent component is unknown. Only parameters 'id', 'rows' and 'cols' are necessary to create a Display.

Once the XDisplay has been created, it starts to live on its own". The XDisplay.class implements MD*Crypt's XQSListener. Assuming that it has 


\section{XQC in Detail}

been registered to the XQServer from its parent class, it obtains information regarding results sent back from the server. An obligatory implementation of the handleServerReply() method filters the relevant XQSObject type 'GRAPHICS' and finally creates a new instance of the XPlot.class.

This XPlot.class (see appendix B.14) represents the heart of generating graphical output. It processes the XQSGraphicsObject that contains information about the data that shall be shown, including further properties such as shape, size and color. A detailed description of components, data and methods offered by the XQSGraphicsObject is part of section 3.3.2. The first piece of information XPlot needs to continue is the type of the data part. XploRe's Displays are not just meant for showing graphics. Common textual output could be shown in a Display as well. Each type of data requires different processing. In the case of graphical data, the minimum and maximum values of the data need to be determined. This information is necessary to calculate the size of the plot. Since an XQSDataObject, which comes as a part of the XQSGraphicsObject, can contain more than one data part, all data parts must be considered. If the data object consists of threedimensional data, the data will also be scaled to a 'unit cube'. Missing this step would lead to "odd" results when trying to rotate the plot - points would "leave" the visible area. For the actual drawing process, Java's 2D API is used. This package offers methods for displaying graphics with outline and fill styles, transforms graphics when they are rendered, constrains rendering to a particular area, and controls the way graphics look when they are rendered. If the XQSDataObject contains more than one data part, it will be processed one after another. Output of type 'text' is displayed in a font size that depends on the size of the Display. Before graphical output can be displayed, information about shape, size and color of the data point need to be extracted from the XQSDataObject. The actual drawing of the data point itself is realized within a 'switch-case' statement. Figure 4.32 shows a small extract of the XPlot.class, which demonstrates the 'birth' of a single graphical point.

The variable 'pLook' contains information regarding the appearance of the data point. This information is part of the XQSDataObject that MD*Crypt has created using the server's results. A complete overview of possible shapes is part of section 3.3.2. In case 'pLook' contains a ' 0 ', no graphical output is required for the data point; ' 1 ' means a simple point; '2' means a nonfilled rectangle; ... ; '4' means a non-filled triangle. All shapes are realized using Java's java.awt.geom. ${ }^{*}$ package. For example, a simple point is an 


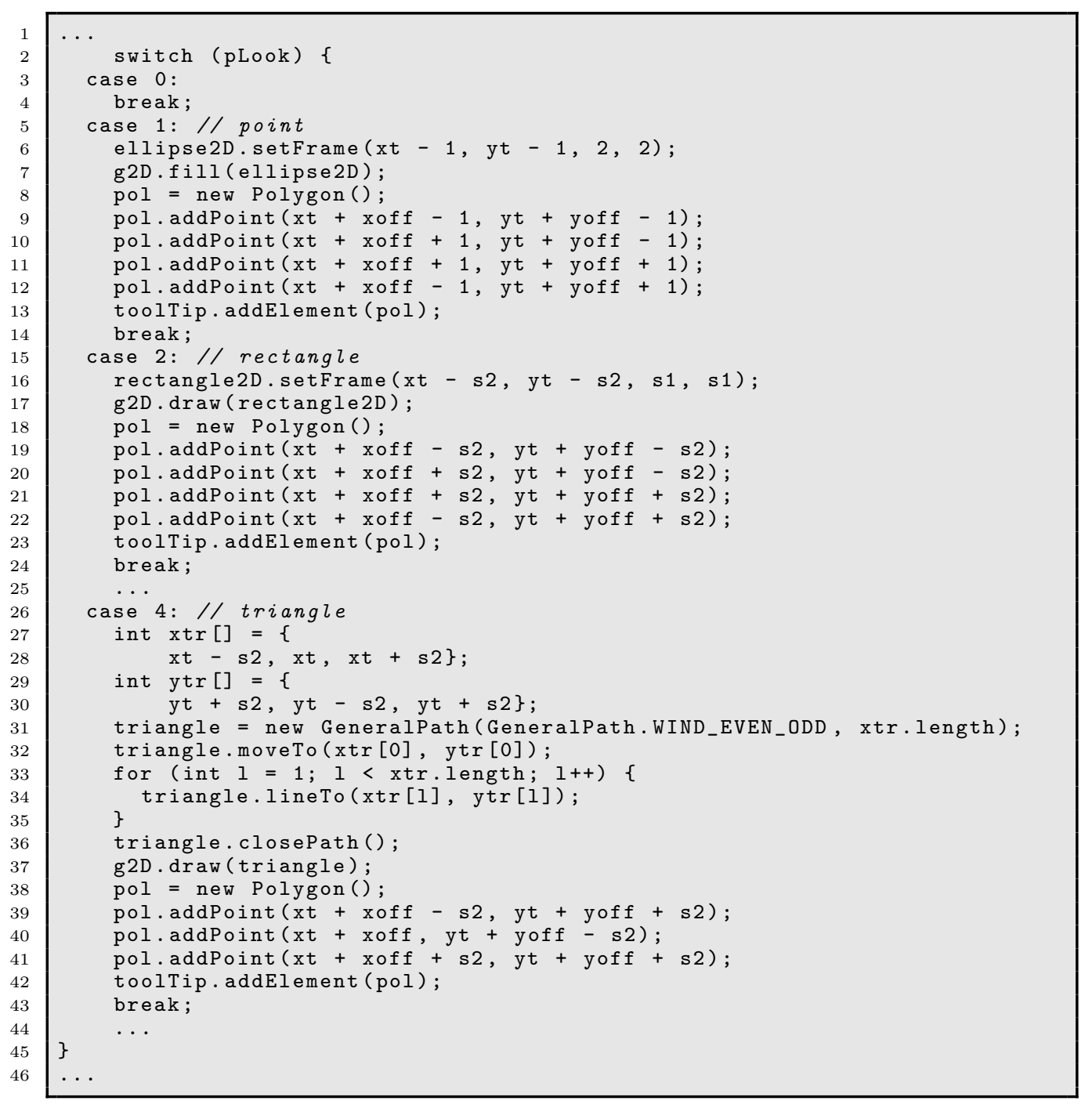

Figure 4.32: Drawing data points

implementation of the Ellipse2D.class; a rectangle is a new instance of the Rectangle2D.class. Using these classes makes it quite easy to create the graphical output. All it requires is information regarding the coordinates ('xt' and 'yt') and the size ('s1' = size, 's2' = one half of 's1') of the shape to be drawn. Slightly more complicated is the realization of shapes such as a "triangle", since there is no direct Java implementation available. Instead the GeneralPath.class can be used. As the name "GeneralPath" implies, this class allows for drawing a line along a stated path. Creating a triangle is 


\section{XQC in Detail}

accomplished by drawing a path that includes all three corners of the triangle.

Section 4.1 .8 contains a description of additional features offered by the Display. One of those features is the possibility for showing coordinates of a data point within a plot by simply pointing the mouse over the shape (see figure 4.24). To realize this feature, we have taken the opportunity to create a tool tip text for many Java components. A tool tip text is a text that is shown if a pointing device stays over any point of the component that has implemented this feature. Unfortunately, this possibility is not offered for graphical Java components like Ellipse2D or Rectangle2D. One of the Java components that offers tool tip texts is a javax.swing.JPanel, which also works as a basis for the XPlot.class. Since a tool tip text should only be shown if the pointing device stays over a certain data point, but the data's coordinates are of course not identical, an additional logic is required for realization. For this purpose we use two variables - 'pol', which implements a java.awt.Polygon and 'toolTip' as a common vector. Every time a data point gets drawn, the XPlot.class also creates a polygon with the same coordinates and the same size as the original data point, and appends it to the vector 'toolTip' (see figure 4.32). Variables 'xoff' and 'yoff' consider a shifting of coordinates due to axes that are also shown within the plot. As result, the vector contains information about the representation of every data point on the plot. The vector is used within the method checkForToolTip (int xtt, int ytt) that is part of the XPlot.class. This method is called by the event handler class XPlotAction.class - every time the pointing device gets moved over the plot passing over the actual coordinates. If the actual coordinates are inside of one of the polygons of the vector 'toolTip', a tool tip text containing the data point's coordinates will be shown.

Another important feature the XPlot.class offers is the possibility to rotate three-dimensional plots. To realize this feature, the four following matrices play a significant role:

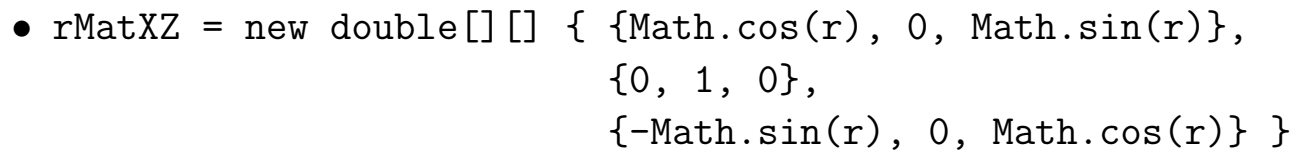

... contains settings for rotating a plot to the right.

- $\operatorname{rMatZX}=$ new double [][]$\{\{$ Math. $\cos (r), 0,-$ Math.sin $(r)\}$, 


\author{
$\{0,1,0\}$, \\ $\{$ Math. $\sin (r), 0$, Math $\cdot \cos (r)\}\}$
}

... contains settings for rotating a plot to the left.

- rMatYZ $=$ new double [][]$\{\{1,0,0\}$,

$$
\begin{aligned}
& \{0, \text { Math. } \cos (r), \text { Math } \cdot \sin (r)\}, \\
& \{0,-\operatorname{Math} \cdot \sin (r), \operatorname{Math} \cdot \cos (r)\}
\end{aligned}
$$

... contains settings for rotating a plot upwards.

- $\operatorname{rMatZY}=$ new double [][]$\{\{1,0,0\}$,

$$
\begin{aligned}
& \{0, \operatorname{Math} \cdot \cos (r),-M a t h \cdot \sin (r)\}, \\
& \{0, \operatorname{Math} \cdot \sin (r), \operatorname{Math} \cdot \cos (r)\}\}
\end{aligned}
$$

... contains settings for rotating a plot downwards.

- Mat $=\{\{1,0,0\}$, $\{0,1,0\}$, $\{0,0,1\}\}$

... contains settings for showing the default X-Y level.

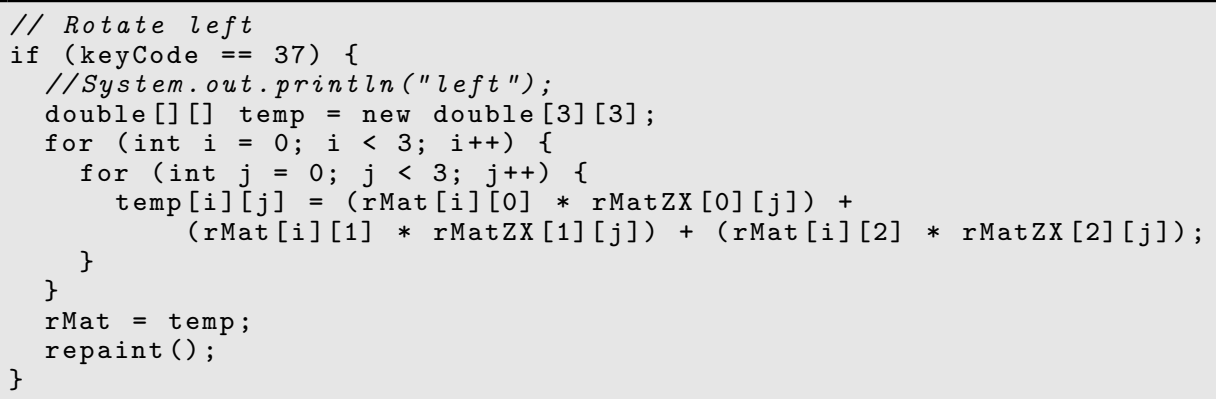

Figure 4.33: Calculating the rotation matrix

All four variables contain information, which is used within the rotation process. Parameter ' $r$ ' determines the degree of rotation. The actual rotation process is realized within the method rotatePlot(int keyCode, double 


\section{XQC in Detail}

r) that is part of the XPlot.class. Two possible actions, performed within the plot, work as a trigger for rotation: dragging a pointing device such as a mouse, with the left mouse button pressed, or just using the keyboard's arrow keys. Both events are interpreted by the XPlotAction.class, which handles all events concerning the XPlot.class. The parameter 'keyCode' determines the direction the user wants to rotate the plot (for mouse-dragging, the direction is translated to a key code direction). Rotating a three dimensional plot takes place in two steps: calculating the rotation matrix and calculating the rotated data points. Figure 4.33 shows a small extract of the XPlot.class, which calculates the rotation matrix for rotating the plot to the left.

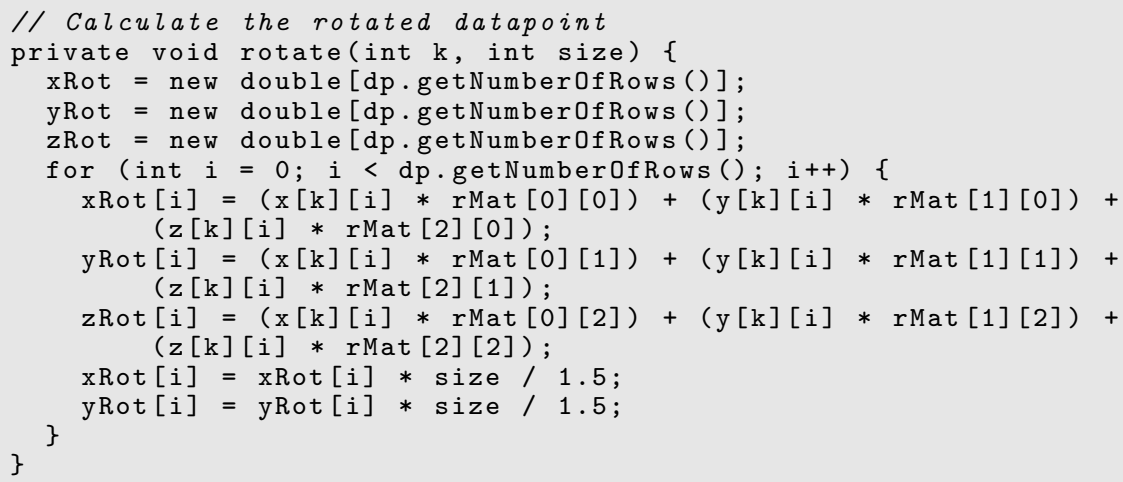

Figure 4.34: Calculating the rotated data points

The rotation matrix is needed to calculate the new coordinates of the data points within the plot. Every data point gets multiplied with the corresponding value of the rotation matrix rMat. Result are rotated data points (xRot, yRot and zRot) that are shown after repainting the graphic.

In its current version no communication takes place between the GUI components Method/Data Window and Display. A challenge for future work would be to offer the possibility to manipulate data via GUI components and send those manipulations to the server or to other GUI components. An example would be the marking or brushing of outliers within plots in order to have them automatically be marked in the spread sheet as well. 


\section{READ VALUE / SELECT ITEM}

The XploRe Quantlet server offers two dialog objects, which are part of the XploRe programming language: "read value" and "select item". Both components allow for communication between user and server out of a running XploRe Quantlet.

Read Value does exactly, what its name implies. It asks the user to pass a value (or values) to an XploRe Quantlet. This value can then be used within the Quantlet. Read value is realized by the XReadValue.class (see appendix B.17). It extends Java's javax.swing.JDialog to generate a modal dialog window with the XQC's Desktop Window as its owner. A modal window is necessary because the server is waiting, expecting a certain type of data stream - the result of the Read Value request. Key object of the communication is MD*Crypt's XQSReadValueObject. It does not only contain names and default values, the XploRe program is asking for, but it does also provide a method to send the values back to the server immediately. In order to react to the user's input, the XReadValue.class has implemented the required ActionListener.

Select Item works in a similar manner as Read Value. Instead of expecting the user to enter values he/she is asked to select an item from a list. The XSelectItem.class (see appendix B.18) also generates a modal dialog window, but in this case it holds an instance of Java's javax.swing. JList. It waits for the user to choose one or more items and to release this selection. MD*Crypt's XQSSelectItemObject contains a list with names the user can select from. The result of the selection is then send back to the server as an array that contains the status (checked or not checked) of the list items. 
XQC in Detail 


\section{Chapter 5}

\section{XQC/XQS Compared to Other Web Based Statistical Solutions}

Searching the Internet for net-based statistical solutions leads to three different approaches:

1. CGI techniques,

2. "Standalone" Java applets,

3. Java based client/server computing.

The following section attempts to compare the different approaches and to find their advantages and disadvantages.

\subsection{CGI Techniques}

Using CGI techniques, the user enters data or the location of a data file via a CGI interface. A statistical program on the server side calculates and sends back the results to the user. The user will get the results either right away, shown in the browser window, or the result will be sent to the user by e-mail. Examples are given by Inoue et al. [IAYY01], the $M M M$ project (http://macke.wiwi.hu-berlin.de/mmm/) - see also Günther et al. [GMK ${ }^{+} 97$ ] - and the Rweb project (http://www.math.montana.edu/Rweb/). 


\section{XQC/XQS Compared to Other Web Based Statistical Solutions}

Advantage of the CGI approach is the use of an architecture that is similar to the client/server architecture. With the statistical program running on the server side, the user can access resources of a powerful computing system as offered by our XQC/XQS approach. Further advantage of CGI is its pure HTML interface. Since it does not relay on Java or any other runtime environment, it runs without the requirement of installing any plug-in software.

While CGI remains a popular tool for Web applications, it has some important limitations. CGI has performance bottlenecks - each HTTP request to a traditional CGI program, usually results in the creation of a new heavyweight process at the Web server. Once the process is finished, it goes away. Fast CGI can offer a solution to overcome performance limitations. Fast CGI (http://www.fastcgi.com) works by starting a process, which keeps running in memory. Thus it is possible to process one request after another, instead of having a new process started for each request. CGI is a simple interface that offers no support for high-level system services, such as scalability, load balancing and resource management [SSJE02]. Maintaining state is a difficult task within this type of architecture. A CGI application like Rweb cannot change its interface with the underlying environment or as reaction to different tasks.

\section{2 "Standalone" Java Applets}

Interactivity is an advantage offered by the use of Java applets. Most statistical (standalone) applets available via the World Wide Web have two things in common - they are completely programmed in Java and integrated in one single applet. Therefore, the user has to download the whole program containing the computation algorithm, as well as routines for presenting the results. Examples of such statistical applets are the Internet Statistical Computing Center (http://www.statlets.com) and the StatCrunch project (http://www.statcrunch.com), see West et al. [WWH04]. Our XQC/XQS architecture on the other hand, splits the load between the client and the server. The Java client only has to present the output computed by the server. Therefore it can be a relatively slim application.

For calculating a simple histogram of a small dataset, the use of a single Java applet would be appropriate. But the more complex a statistical algorithm gets, and the larger the datasets become, the more difficult becomes a 


\subsection{Java Based Client/Server Computing}

pure Java implementation of these algorithms, and the more load lies on the client computer. Computing a nonparametric time series process that contains approximately a thousand observations within a single Java applet is hardly possible. The computational load of the XQC/XQS model lies on the server side, which can take advantage of the powerful underlying computer architecture. This speeds up the computational process significantly. Due to the communication process which takes place between client and server, the XQC might take slightly longer to calculate simple statistical problems as compared to a pure Java applet. But with increasing complexity, the time saved using the server power exceeds the time needed for the communication process.

Expanding an existing Java applet with a new statistical method implies an extensive effort reprogramming the method. The new method, usually developed using a statistical software package, would have to be reprogrammed in Java. Extending the XQC/XQS model would only require adding the new method programmed in XploRe to the server's or to the client's methodbase without any reprogramming effort on the client or server side.

\subsection{Java Based Client/Server Computing}

In addition to our XQC/XQS project, there exist other projects using client/server approaches for statistical computing via the Internet. One example is the Java based Statistical Processor - Jasp project (http://jasp.ism.ac.jp/index-e.html), see Kobayashi et al. [KFYN01]. Jasp is a statistical system whose language is based on Pnuts (http://javacenter.sun.co.jp/pnuts/). Similar to the XQC/XQS model, the Jasp approach uses the advantages of Java, and Java applets respectively, to implement a user interface. The mixed user interface consists of a character user interface (CUI), as well as a graphical user interface (GUI). But the XQC is not meant to only work like a "conventional" program. The advantage of the XQC is the possibility for customizing its behavior via configuration files. This characteristic offers a way to extend the features of electronically enhanced books (e-books) toward interactive examples. The Jasp approach allows for distributed computing on several servers; whereas, in the XQC/XQS model, a client chooses a certain server that computes the data of this (and possibly other) client(s) during the entire session. 
Another example for a Java based statistical computing environment is the Omega project (http://www.omegahat.org/). The aim of this project is to provide a variety of different modules (GUI, Graphics, CORBA, language, numerical methods, etc.) that can be combined by the user to meet his/her particular needs. Distributed computing is supported by the integration of the CORBA interface, which provides access to remote servers. Of particular interest for the Java programmer is the org.omegahat.R.Java package, which provides several methods for evaluating $R$ code directly in Java, and getting back the results of the evaluation. For an overview of the available features, see Temple [Tem02]. An integration of this package into the MD*Serv middleware can extend our architecture to handle $R$ requests. In addition to the interaction between $R$ and Java, omegahat offers another means for accessing $R$ resources via the Web, namely the $R$-plug-in for Netscape. This plug in allows for calling $R$ from JavaScript. Unfortunately, it is only available for Unix operating systems.

Pure Web based client/server approaches suffer from the well-known problems of the Internet - the security of data transferred via the Internet and the stability and speed of the network/modem connection that may represent the bottleneck of the client/server architecture. Encrypting the data could solve the security problem. To take advantage of the server's speed, a fast and stable network/modem connection is required for the transport of data and results. The technical development in this area should help to solve this problem in the future. 


\section{Chapter 6}

\section{Reproducible Research Using the XQC/XQS Technology}

In today's world, there hardly exists a field where research is possible without the (mostly extensive) use of computers. "Some of the recent advances in statistics have even been dependent on advances in computer science and technology. Many of the currently interesting statistical methods are computationally intensive, either because they require very large numbers of numerical computations or because they depend on visualization of many projections of the data." [Gen03] The financial researcher uses mathematical and statistical models to predict market behavior; the social scientist uses it for research about the deployment of the birthrate in Germany; the physicist uses statistics for modeling the influence that temperature has on the conductance of copper.

It is desirable for an reader to be able to verify and validate the published results of statistical research. Even more desirable to an interested researcher, is the ability to inspect the source code, modify it and produce variations of the results. Buckheit and Donoho [BD95] outline the topic of Reproducible Research - "An article about computational science in a scientific publication is not the scholarship itself, it is merely advertising of the scholarship. The actual scholarship is the complete software development environment and the complete set of instructions which generated the figures." They propose the publishing of research papers as electronic books, with the inclusion of the software environment the results were generated with, to make them interactively accessible. Claerbout [Cla02] defines the purpose of reproducibility 


\section{Reproducible Research Using the XQC/XQS Technology}

as a possibility "... to facilitate someone going a step further by changing something ..." - to extend the author's results.

Over the past several years, a number of approaches have been developed that integrate computations and analysis into documentations. For example, ReDoc concept [CKS00], embedding $R$ programs into Netscape [GTL03] and the Sweave project [Lei02], which combines $\mathrm{HT}_{\mathrm{EX}}$ with $S$ programs and makes it possible to create dynamic reports. These reports which can be updated automatically if analysis or data changes.

Reasons for making research reproducible can be manifold:

Buckheit and Donoho [BD95] probably remembered of long nights of work while describing one possible reason: Writing an article that contains lots of figures as a result of research, often takes quite some time. During this period of time, hundreds of figures are usually generated, varying algorithms and parameters. At the end the question might arise: Which figures were the final versions that should be used for the article? Taking "the nicest looking figures" would not be the right choice. Illustrating exactly the figures that were generated using the final algorithms and parameters in the article would be the best choice. They might not be the best looking, but are the true results of the research. Publishing reproducible figures compels the author to use the right graphics and the right results, since every reader is actually able to regenerate this figures.

Consider yourself reading your own paper one, two or even several years later. Would you be able to generate the same picture as in your original paper? If, for some reason, you do not have the original program code hidden somewhere, it is going to be difficult. Even though you would still have the software code, the corresponding environment might have been changed in the meantime, making it impossible to reproduce your results. Using new media like CD-ROM, makes it possible to publish not just the written paper, but also the software, or parts of it, that had been used for research.

Thinking of a young student who begins to do scientific research leads to another reason. Often times students face the problem of finding the right initiation into their topic. Although they are usually able to access tons of literature sources, most of them are "limited" to merely explaining algorithms. Rarely, the used parameters are included in published papers. In order to use those algorithms as a starting point, the student attempts to reproduce the previous result. This means programming the entire logic again to use it as the basis for further study. Reproducible research can help to 


\subsection{Types of Presentation}

make the entry into the world of scientific research much easier for young students. They would actually be able to try the programs, change and vary parameters, or even extend existing programs during their study. On the other hand, being able to publish results within interactive documents allow those young researchers to more easily share their results and knowledge with other scientists.

To summarize, research reproducible can:

- Make research more accurate and more credible, since other researchers are able to discover any errors and to validate the results.

- Save time for other researchers working on the same or a similar task, since their research can be built upon validated and expandable results.

- Most likely help young students beginning their research.

Presentation of research results is usually limited to formulas and graphics, and their description and additional explanations. The main reasons for this limitation is the austerity of the medium that is most often used - common paper. But the quickly growing Internet and dropping prices for electronic media, such as CD-ROM and DVD-ROM, have begun to change the dominance of paper. These Media allow for presentations that exceed the message a simple graphic can deliver.

\subsection{Types of Presentation}

How can the results of scientific research be presented to the audience? What are the advantages and disadvantages of the different ways? The following section attempts to find answers to these questions. To illustrate the different forms of representing scientific content, a very simple example from basic statistics will be used - analyzing the coherence for decathletes between the results of $100 \mathrm{~m}$ and long jump. As simple as this example may seem, it often works as a basis for further research and more complex algorithms. The more complex the methods and algorithms of a mathematical or statistical research become, the more effort is necessary to prepare and to present the results of this research.

We define different level, or stages respectively, for presenting statistical and mathematical results within research documents. 


\subsubsection{Text Only}

The simplest form for presenting results of scientific research is a pure textual description. This form of presentation is limited to the use of algorithms, formulas and explanations of coherences. A disadvantage of this method is the presupposed ability of any reader to understand the abstract. This ability is often needed in order to understand the content, to put oneself in the author's position. Using the decathlon example we get the following results:

- Correlation coefficient: -0.69,

- Regression function: long jump $=17.1825-0.8988 * 100 \mathrm{~m}$,

- Coefficient of determination: 0.48 .

The data itself can be presented within a table - see table 6.1.

\begin{tabular}{|l|c|c|c|l|}
\hline Decathlete & A & B & C & $\ldots$ \\
\hline $100 \mathrm{~m}$ & 11.25 & 10.87 & 11.18 & $\ldots$ \\
\hline Long Jump & 7.43 & 7.45 & 7.44 & $\ldots$ \\
\hline
\end{tabular}

Table 6.1: Results of a decathlon

What are the results of this simple research? Interpreting the calculated results leads to the conclusion that there seems to be coherence between the two sports. But without taking a closer look at the data itself, and/or calculating additional coefficients a "final" conclusion is hardly possible. The dataset could, for example, contain outliers that influence the results. It requires additional numbers and explanations to get an image of the actual coherence. Many readers of those types of publications try to transform those pure numbers into graphics using their imagination. With this simple example this might be possible. But imagine, for example, the presentation of the results of a kernel regression estimate.

\subsubsection{Graphical Representation}

The use of graphics for presenting the results of scientific research is an extension of the method just described above. Graphics are meant to help 


\subsection{Types of Presentation}

the author, as well as the reader, to recognize and to understand coherences in datasets and to visualize presented algorithms. Figure 6.1 shows a scatter plot of the decathlon example used in the previous section. In addition, the left graph also contains the calculated regression line. This figure simplifies an interpretation of the coefficients calculated above, and makes it easier to identify a statistical coherence between $100 \mathrm{~m}$ and long jump. In contrast, the right part of figure 6.1 shows the same data set with the estimated kernel regression (Nadaraya-Watson regression).
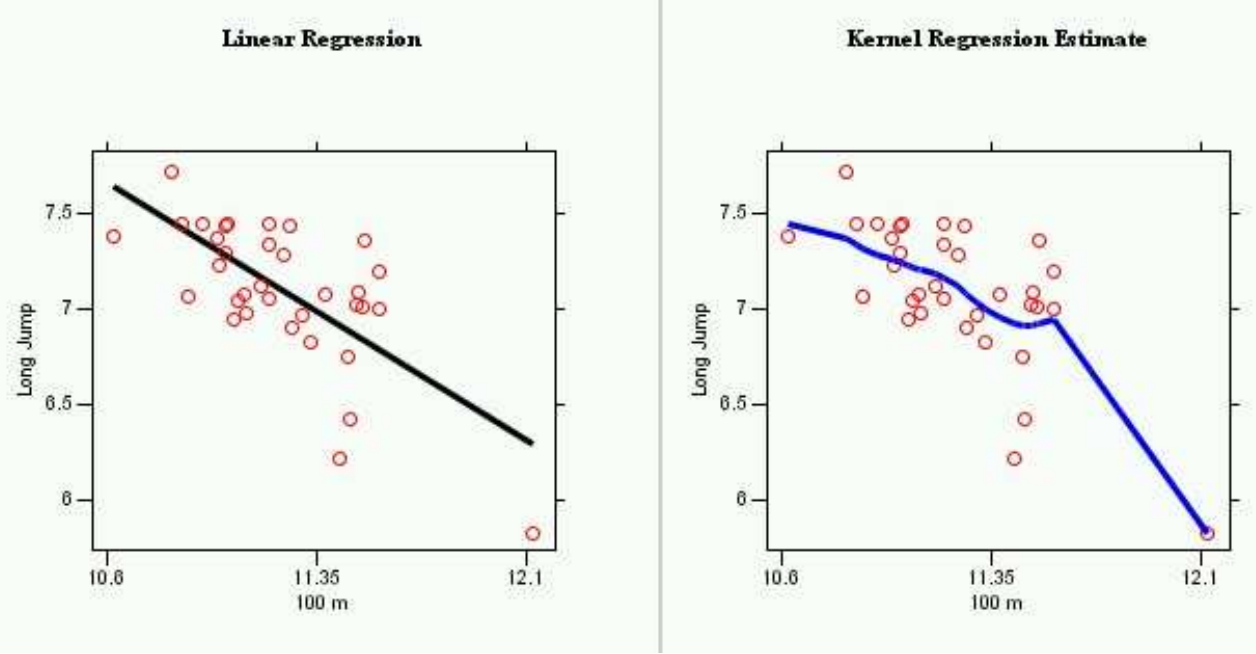

Figure 6.1: Scatter plots with linear regression and kernel regression estimate

'Text only' and 'graphical presentations' of scientific results have one thing in common - it is difficult to determine from where the calculated results and graphics were derived. Are they an outcome of a mathematical or statistical software environment or is the presented graphic the result of a graphical program? We certainly do not want to question published results without the ability to reproduce them. Nevertheless, the reader has to accept that the published results really are the outcome of the author's calculations. An additional publication of used datasets, the source codes of programs used for the calculation of results and the creation of graphics, as well as information regarding the used software environment, are basic approaches for providing an interested reader at least the opportunity to verify and reproduce the results. The pure source code is only useful if the reader has access to the same software, libraries, and methods the author used. But in many cases 
that might not be that easy.

\subsubsection{Reproducibility of Calculated Results and Graphics}

What does reproducibility, or reproducible research, mean? Gentleman and Temple Lang [GTL03] define: "Reproducible research is reproducible in the sense that the author has provided sufficient detail (in the form of code and data) for a reader to reproduce the details of the authors presentation." Adhering to this definition, reproducibility is possible by adding all data and source codes used, to a publication. As already mentioned above this information does not automatically enable the reader to reproduce the research. Without access to the statistical software environment, the author used, it is hardly possible to reproduce any results. In addition, software is often subject to short life cycles in terms of versioning. Even a year can be a long time, making the source codes worthless. "Printing the code, which made so easy to understand and implement the algorithms in many scientific books and articles of the 70's, is unusual now, due to the large number of lines of code. Even if the code itself is known, the precise parameters and input data used to create the figures are missing." [Vla02] For solving this type of problem Buckheit and Donoho [BD95] go one step further - "When we publish articles containing figures which were generated by computer, we also publish the complete software environment which generates the figures." Both citations can be combined to a more abstract definition - reproducibility is defined as the possibility for the reader of a document to re-compute results or re-build figures respectively.

Figure 6.1 was generated using the software environment XploRe. For this example, reproducibility could be accomplished by publishing the source code of the XploRe Quantlet we used. In this case, the reader needs to have access to this software environment in order to reproduce the figure. In today's world, more and more software developers offer access to their software via Web interfaces. Attaching the source code to an article is the easiest way to realize reproducibility. The advance of this approach is the independence of the media from pure paper. There is no need for additional software that would have to be published as well. The drawback of this approach is the limitation that the reader cannot regenerate the figure right away but has to "break out" of the article. 
If the article is published via the Internet or on CD-ROM, a second way for making our figure reproducible would be available: Clicking on a link within the document (PDF or HTML) would open an additional window on the screen where the same figure as published in the article is re-generated. Advantage of this approach is the opportunity to reproduce results without actually having to leave the document itself. The 'additional' window could even provide additional functionality rather than just showing a figure. This functionality could include showing the coordinates of a data point, printing functionality, or - if a three-dimensional plot has been generated - offering the possibility to rotate the plot. The drawback of this approach is the dependence on interactive media.

\subsubsection{Reproducibility and Interactivity Regarding Data}

Interactivity regarding data represents an extension of the simpler form of reproducibility described above. With this kind of reproducibility, readers are not limited to using only the data published by the author, but they have the opportunity to apply the algorithms on their own data. In comparison to the previously discussed approaches, interactivity implies the usage of media rather than paper. Whereas simple reproducibility would be possible by publishing the source code; to offer interactivity, the software environment needs to be published as well. Internet, CD-ROM or DVD-ROM are preferable for publishing in this case. This does not necessarily mean replacing paper. Instead, this should be seen as a supplement rather than a replacement.

Being able to change or vary data enables the reader, who becomes a user, to actually verify research results by playing "What if ..." scenarios. For example, eliminating outliers, helps to understand presented theories. An additional advantage is the possibility of applying new published algorithms to the reader's own data.

\subsubsection{Reproducibility and Interactivity Regarding Data and Statistical Programs}

Even more useful for many readers, would be the possibility of having access to the underlying program that generated the calculations and graphics. This 


\section{Reproducible Research Using the XQC/XQS Technology}

feature not only enables the user to validate the author's results, but the source code can be used as a basis for further research.

For this form of representation the same drawbacks as for the previous methods apply - its usage presupposes a publication on interactive media.

\subsection{Making Research Reproducible}

The approach presented in previous chapters attempts to allow for reproducibility of scientific results, as well as for interactivity in scientific publications. The aim of this approach is to combine:

- Fundamentals of a research (the used data),

- Results (theorems, algorithms, graphical and non-graphical outcome),

- Final conclusions,

within an interactive document.

The XploRe Quantlet Client/Server model consists of the three components: server, middleware and client.

The XploRe Quantlet Server (XQS) would be offering services to one or more client(s). It is based on the statistical software environment XploRe, which offers high-level statistical programming language, as well as a variety of statistical methods. Running on a remote computer, the XQS offers a magnitude of computer power, which many users would not be able to access otherwise. The XQS offers access to a method- and database, which can easily be extended by new statistical methods - XploRe programs (Quantlets), as well as native code methods, e.g. - $d l l$ and -so). For server side communication purposes, the middleware MD*Serv is attached to the XQS. The communication between MD*Serv and XploRe Quantlet Server is realized via standard I/O streams - the middleware reads from the server's standard input and writes to its standard output.

Middleware, running on server's side, as well as on client's side, enables the communication between client and server. A protocol (MD*Crypt), developed specifically for this architecture, is used for this purpose [Feu01]. 
The XploRe Quantlet Client (XQC) represents the front-end of the architecture. The client is fully programmed in Java2, providing an independent and universal usage. The XQC can be used as an application, as well as running as an applet within a Web browser. In addition, it is possible to setup the client to start in certain ways as defined by the author. For this purpose, a special configuration file is used. The configuration file itself is a simple ASCII file containing commands. These commands allow starting the XQC with:

- 'ExecuteCommand' - executing a command,

- 'ExecuteProgram' - executing a XploRe Quantlet,

- 'OpenData' - opening a data set,

- 'OpenInEditor' - opening a XploRe Quantlet.

Using the commands stated above leads to different levels of reproducibility or interactivity respectively.

Reproducibility of calculated results and graphics is the simplest form of reproducibility. This feature can be realized using the command:

ExecuteProgram $=$ file:///C:/../QRegression01.xpl

In this case, the XQC starts and executes the stated XploRe Quantlet immediately, but without showing the XploRe code. Path statements can be maintained as absolute paths, locally (file:///...) or URL address (http://...), as well as relative to the directory in which the XQC was started (XQCROOT/...). As a result, a graphic is generated that is identical to the graphic in the written document (see figure 6.2). Even though it is the simplest form of reproducibility, a graphic can offers some features, via a context menu, the pure written document cannot. For example, coordinates of the data can be shown and for 3D-plots, rotation via cursor keys or mouse is possible.

If parameters have been used for the computation of results or the generation of graphics, the author has the choice of extending this form of reproducibility by utilizing interactive components the XploRe programming language offers. Using XploRe's "select item" or "read value" for the Quantlet, the 


\section{Reproducible Research Using the XQC/XQS Technology}

author can offer the opportunity to the reader of changing and adjusting parameters and/or to influence the results and the graphics of the computation (see XploRe's APSS - http://www.i-xplore.de/help/_Xpl_Start.html for additional information).

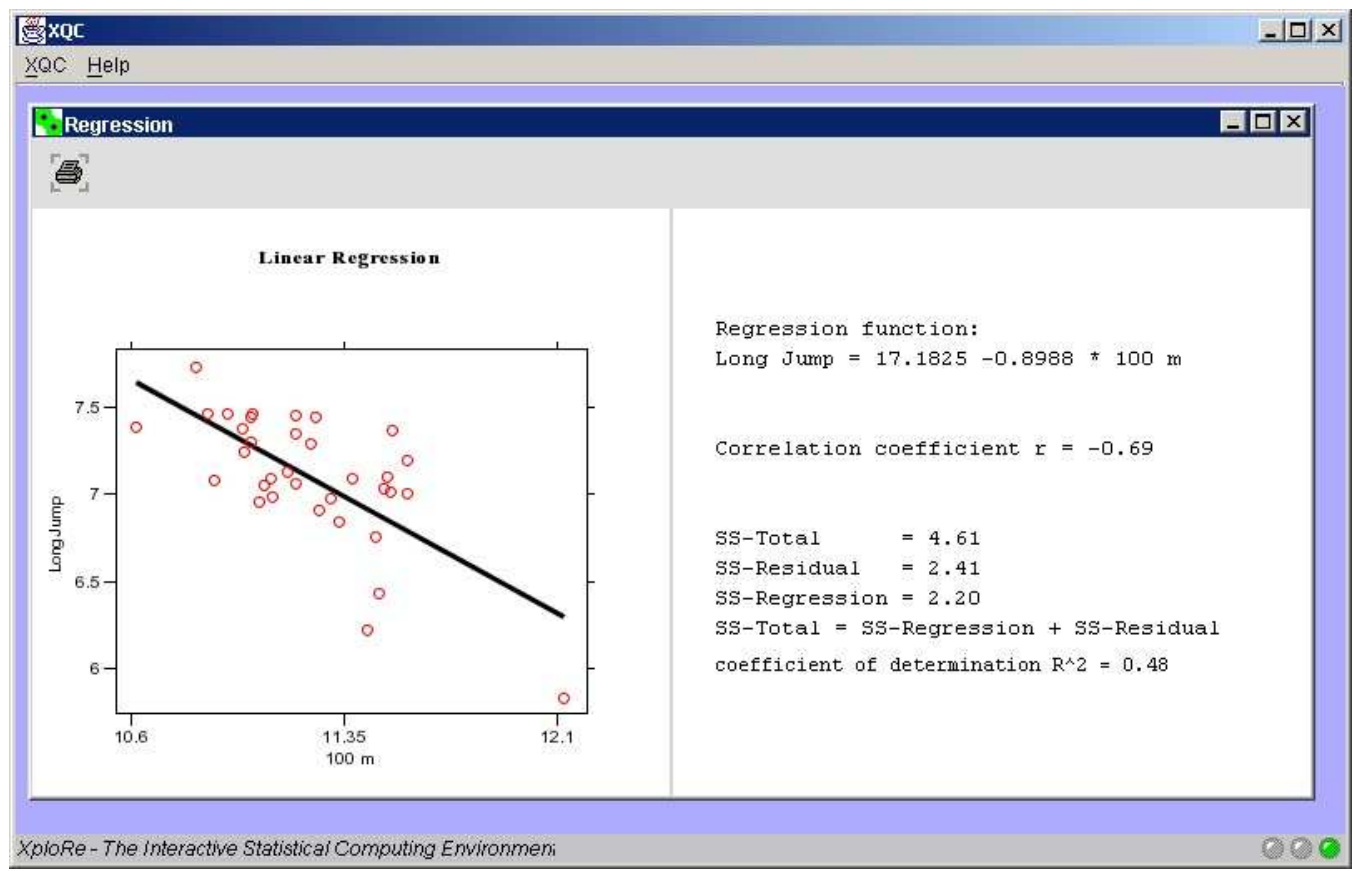

Figure 6.2: Reproducible research

For realizing reproducibility and interactivity regarding data, the following parameters need to be defined in the configuration file:

OpenData $=$ XQCROOT/decathlon.dat

ShowMethodTree $=$ yes

MethodTreeIniFile = xqc_regression.ini

MethodPath $=$ XQCROOT/Xqc_quantlets $/$

An additional configuration file, for this example $x q c \_r e g r e s s i o n . i n i$, defines the XploRe Quantlet, for this example QRegression_02.xpl, that is applied to the data. The Quantlet to be used is stored in a subdirectory ' $x q c_{-} q u a n t l e t s / '$ relative to the directory in which the XQC was started.

Content of the configuration file xqc_regression.ini: 


\subsection{Making Research Reproducible}

Child_1 = QRegression02|Regression

The first part, 'QRegression02', defines the name of the Quantlet and its main procedure. The second part, 'Regression', represents the name that is shown within the Method Tree.

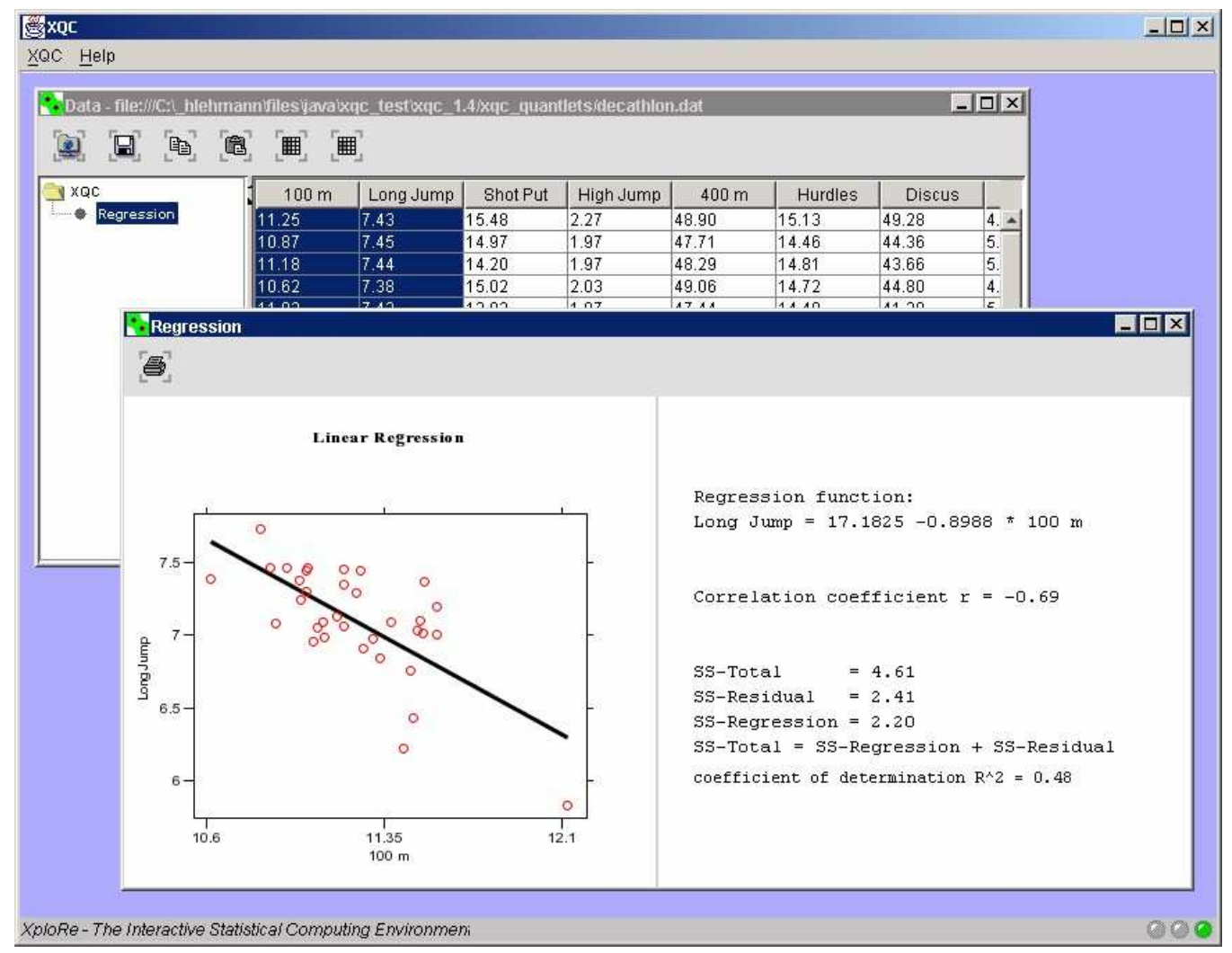

Figure 6.3: Interactivity regarding data

Using the parameters stated above, the XQC starts by opening the stated data set (decathlon.dat) and a method tree that contains the predefined method (Regression), see figure 6.3.

With this form of representation, the reader is not only able to verify the data, but also to change data and explore how this affects the regression coefficients and the graphic. Going one step further, the reader would even be able to use his/her own data for computation.

An extension of the approach described above is the possibility of editing the actual XploRe Quantlet that computed the results. Using the parameter 


\section{Reproducible Research Using the XQC/XQS Technology}

OpenInEditor $=$ file: $/ / / \mathrm{C}: / \ldots /$ test $1 . \mathrm{xpl}$

enables this feature. Immediately after the XQC has been initiated an editor window opens containing the code. The reader can explore, edit and execute the program. If the parameter 'OpenInEditor' is used, in addition to the parameter 'OpenData', the user can edit both - program and data. Altered data can be uploaded to the server and used within the Quantlet. Figure 6.4 shows a screenshot of the XQC offering interactivity regarding data and program.

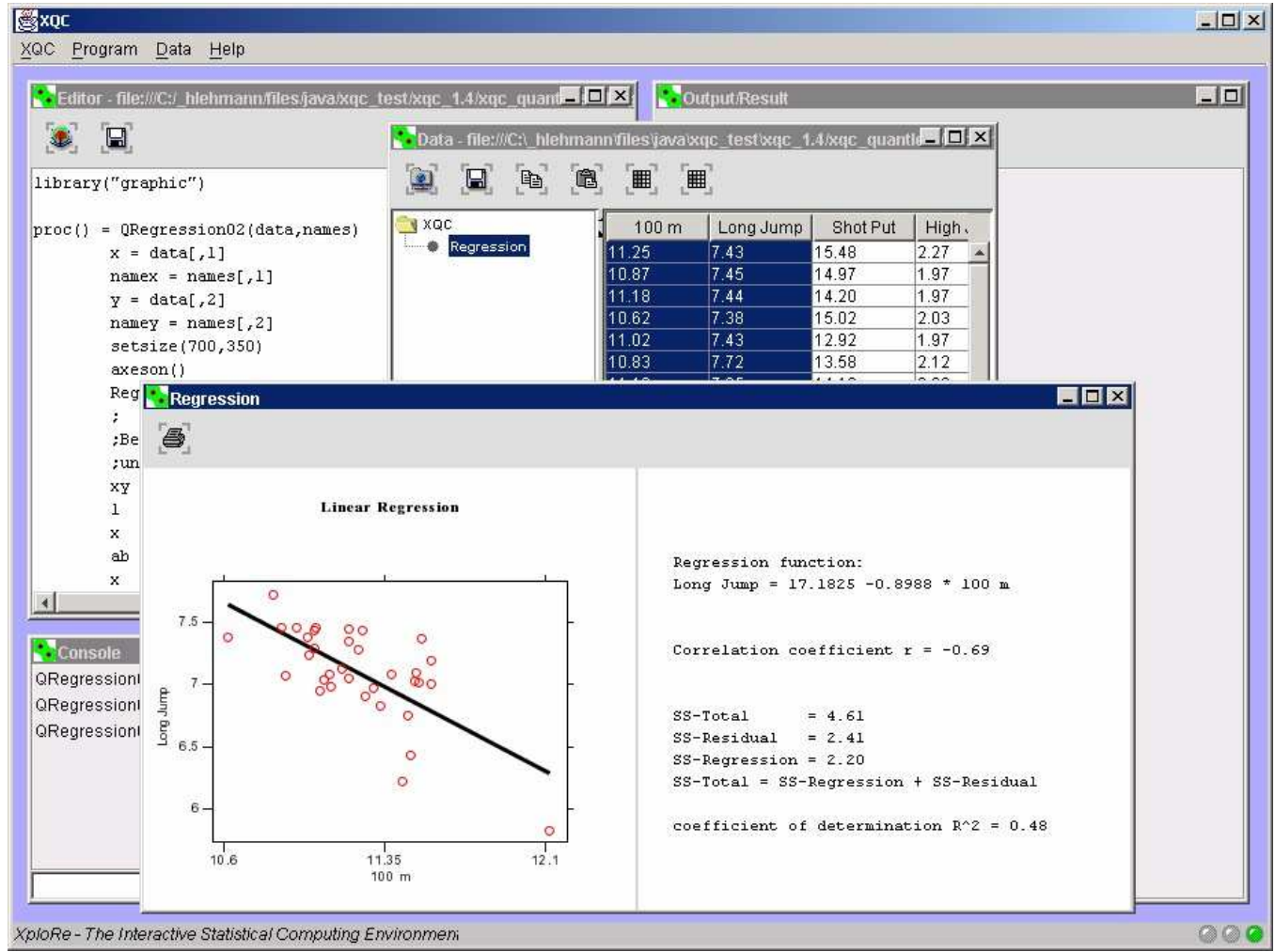

Figure 6.4: Interactivity regarding data and program

With this form of reproducibility, the reader has virtually limitless possibilities for verifying research results. The reader is not limited to the program and data given by the author, but can use his/her own data and program extensions. Therefore, this could be a potential start point for ongoing research in the same area. Researches would not have to start from the beginning. 


\section{3 $\mathrm{MD}^{*}$ Book}

As seen above, using the XQC's functionality to make research reproducible does not require any Java programming skills for adjusting the client. Every XploRe Quantlet the author has used for his/her research can easily be made available for reproduction by just maintaining some parameters in ASCII style configuration files. The final integration into research documents will be explained in the next chapter.

\subsection{MD*Book}

The client/server structure of XploRe allows for an easy integration of XploRe programs (Quantlets) into Web pages.

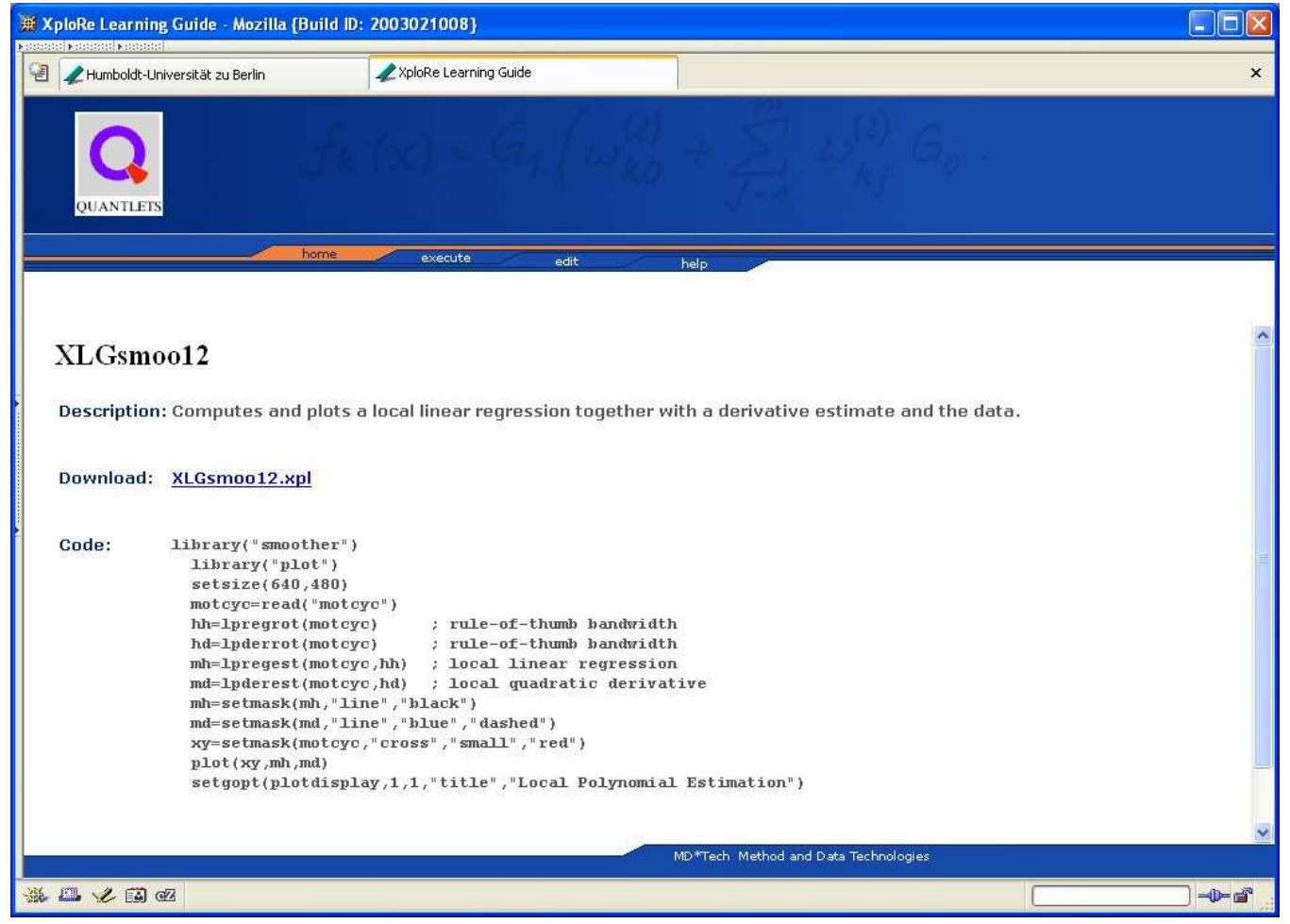

Figure 6.5: Example of reproducibility generated using MD*Book

To have an architecture that actually offers different variants of reproducibility, a technique is necessary for using the advantages of this architecture in an easy way. Of course, if one knows how to program HTML, embedding our client should not be a problem. But in practice, this would mean writing an 


\section{Reproducible Research Using the XQC/XQS Technology}

article using a familiar text processing program, translating it to HTML, and finally working through the HTML document to add the code that allows for reproducibility and interactivity. In most cases, $\mathrm{LT}_{\mathrm{E}} \mathrm{X}$ is the preferred tool for writing scientific articles. In addition there are good translation programs for converting $\mathrm{LT}_{\mathrm{E} X} \mathrm{X}$ to formats such as PDF and HTML (e.g. dvips, pdflatex, latex2html and ghostscript).

With MD*Book, we offer a tool for easily generating electronic books (ebooks) in both formats - PDF and HTML, see Witzel and Klinke [WK02].

Figure 6.5 shows an example of an HTML document offering reproducibility, as well as interactivity, embedded into a Web page. We call this methodology the "Golden Solution". It was generated using techniques offered by MD*Book. The example shows a short description of what the Quantlet "XLGsmoo09" is doing. A link to the source code allows for downloading it, and thereby making the XploRe code available for inspecting and for further utilization.

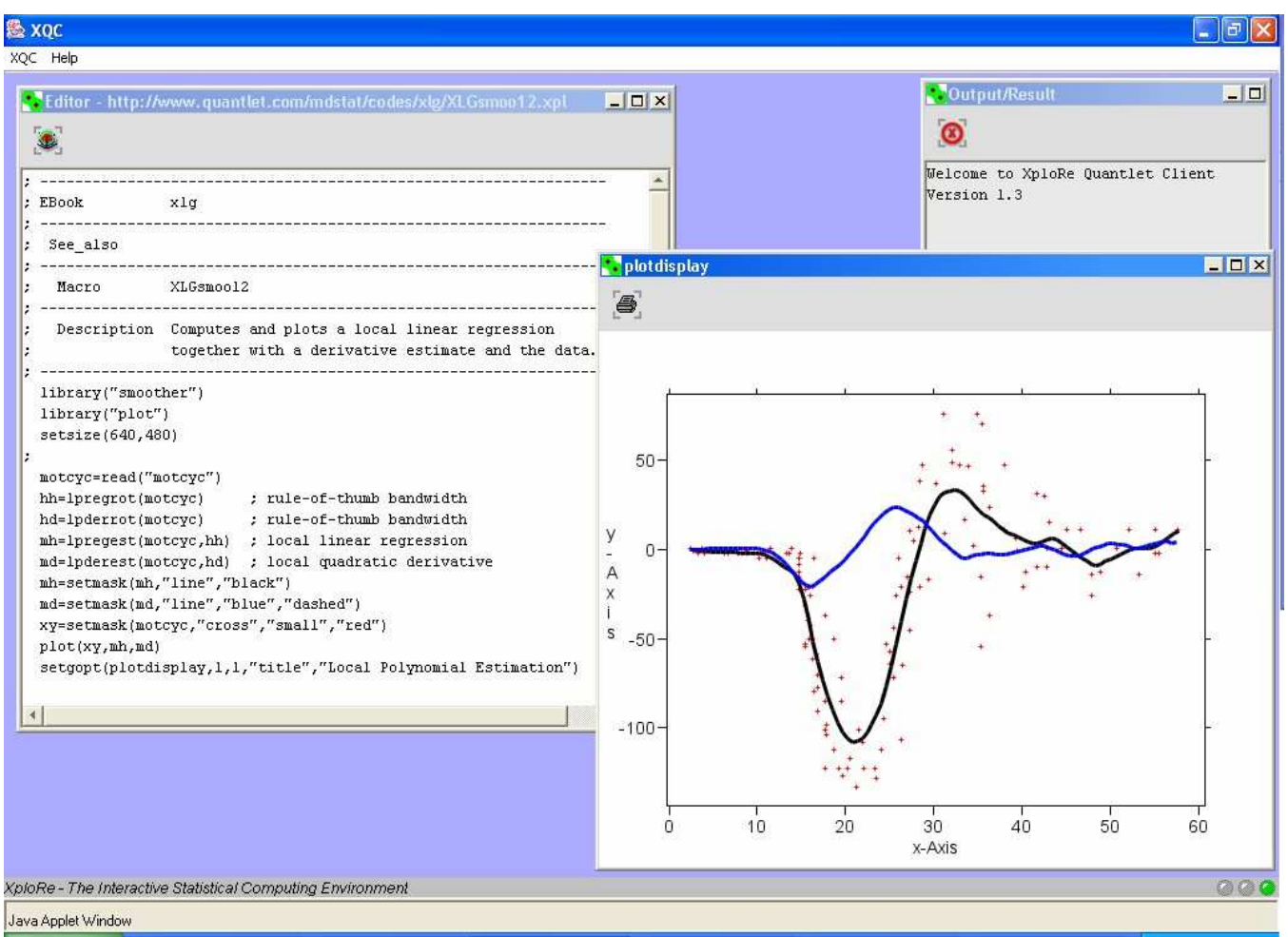

Figure 6.6: Interactive example 
The tab strips "execute" and "edit" allow alternate access to the source code. An inexperienced user would choose "execute", which will simply execute the code (simple reproducibility). An experienced user might choose "edit", which first shows the source code in the XploRe editor window. Now the user can modify the code or run it (see figure 6.6).

To ensure that the code on the Web page and the source code at the server were the same was one of the reasons for developing MD*Book.

At the heart of the MD*Book tool are two developed programs - "tex2sk" and "mdbook". The program "tex2sk" decomposes a set of $\mathrm{AT}_{\mathrm{E}} \mathrm{X}$ files and generates a control file. This control file is used within the program " $m$ dbook" to generate all necessary information. It also contains information about the medium in which the e-book has to be created (e.g. CD-ROM, Internet). An additional set of parameters in the control file, as well as in the $\mathrm{HT}_{\mathrm{E} X} \mathrm{X}$ file itself, allow for the control of various aspects of the outline and appearance of the final document.

The program "mdbook" offers five main options: -ps, -pdf, -html, -java and $x p l$ (see figure 6.7) As the names of the options imply, a PostScript document, a PDF document, or an HTML document, respectively, will be generated. Whereas the option "-html" creates HTML documents without the use of JavaScript, the option "-java" takes advantage of JavaScript functionalities. The option "-xpl" generates the "Golden Solution" for XploRe Quantlets as shown in figure 6.5.

To integrate for a figure, the generating program requires only the integration of a link to the appropriate Web page with the program.

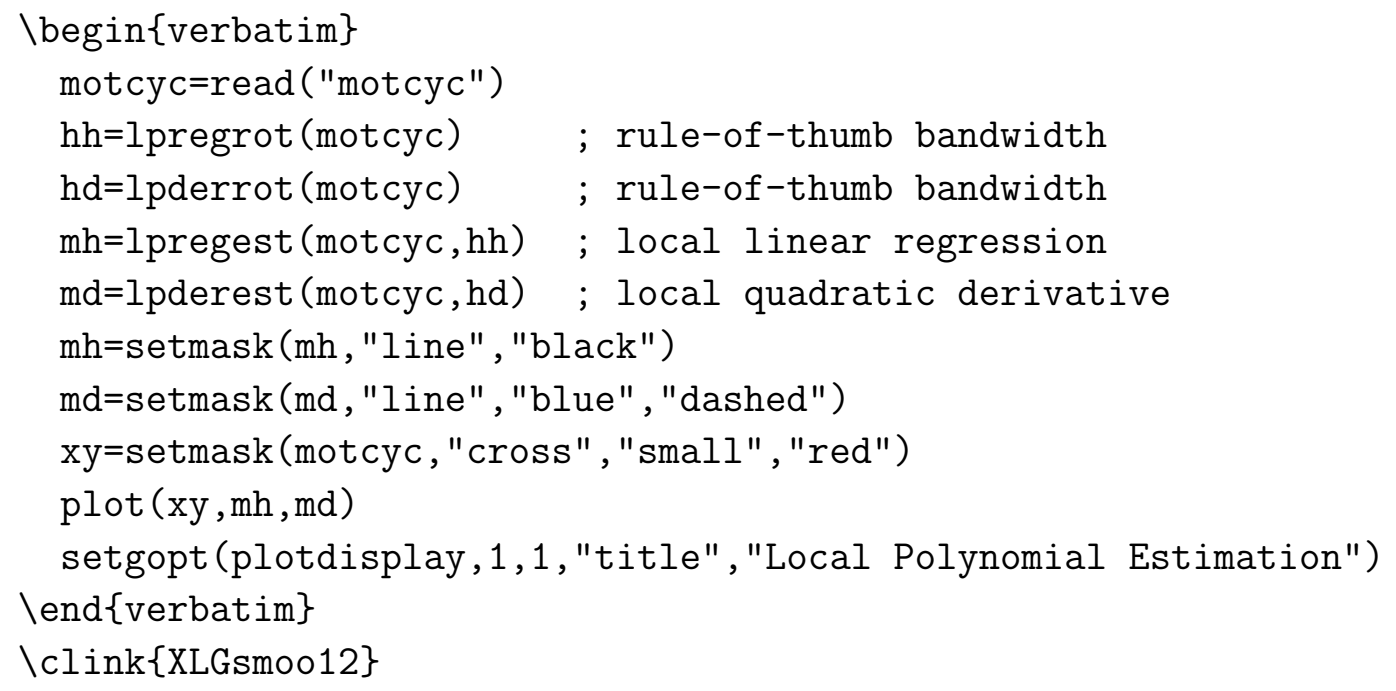




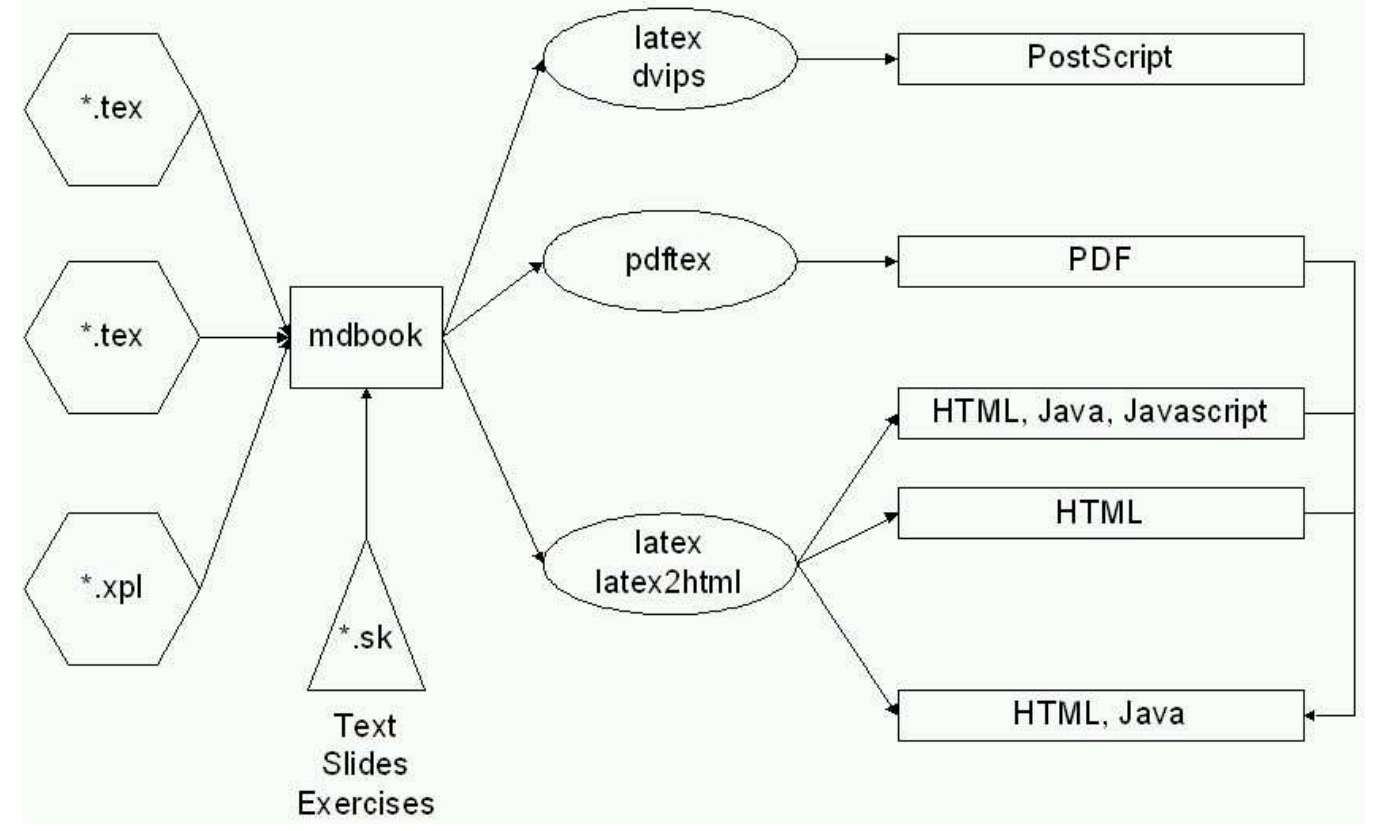

Figure 6.7: MD*Book project

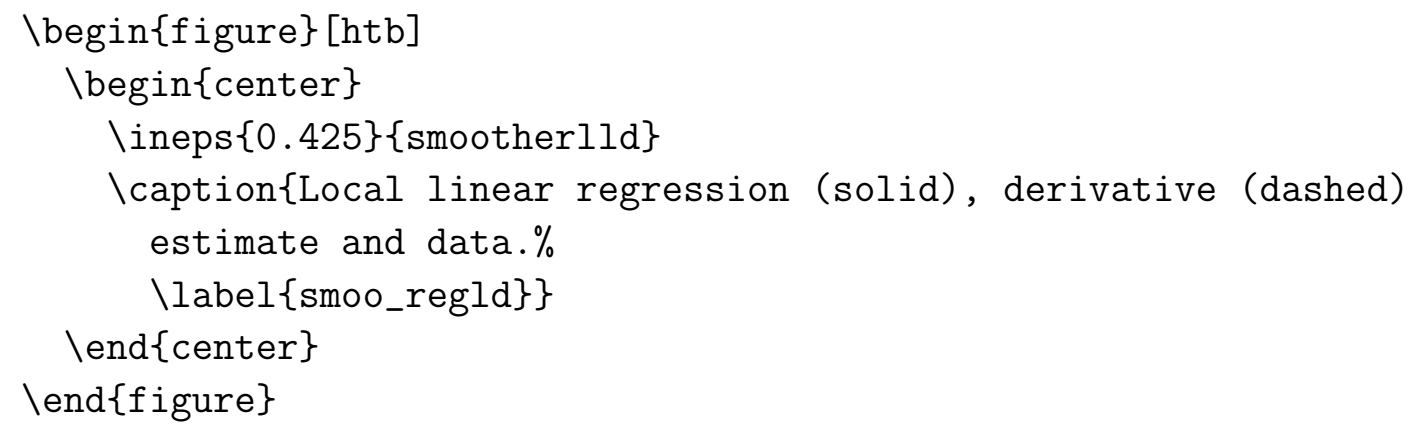

Both architectures - XQC/XQS model as well as MD*Book - have already been in use for quite some time now. Several electronic books have been developed. A collection of electronic books can be found at http://www.ixplore.de/e-books/. A few of them are available for free download, and others have been published in co-operation with Springer-Verlag. 


\section{Chapter 7}

\section{Interactive Teaching - MM*Stat}

Introductory statistics courses are often considered to be difficult by students. Statistics requires a variety of skills, including handling of quantitative data and graphical insights along with mathematical abilities [HR02]. To address this demand, the current teaching methodology, consisting mainly of blackboard work, overhead transparencies and textbooks, has to be revised. Interactive teaching tools are an attractive and powerful way of providing statistical content to students. Many statistical teachware packages and electronic textbooks have been developed during the last several years. Some of them are available for free via the Internet, without any registration requirements. An examples is HyperStat (http://davidmlane.com/hyperstat/) by David M. Lane [Lan99]. Other tools, such as WebStat (http://www.webstatsoftware.com), are commercial teach-ware packages that require registration without charging any fees. A third group of tools requires payment for online access, e.g. CyberStats (http://www.cyberk.com), or the package is only available on CD-ROM such as ActivStats (http://www.datadesk.com). For an extended review of CyberStats, ActivStats and $M M^{*}$ Stat can be found in Symanzik and Vukasinovic [SV02]. 


\subsection{Introduction}

$\mathrm{MM}^{*}$ Stat is a flexibly applicable tool for supporting the teaching and the learning process for statistics in basic studies. It is available in different languages (English, German, French, Spanish, Italian, Czech, Polish and Indonesian) via the Internet (http://www.md-stat.com), and also published on CD-ROM. The development of $\mathrm{MM}^{*}$ Stat was influenced by the achievement of the following goals:

1. Training sessions must provide a broad spectrum of concrete and practical areas of applications of statistical techniques for students coming from different fields. A purely theoretical presentation is often felt by students to be "boring".

2. Statistical analysis in real life can hardly be processed without the use of computers. A training session should therefore contain the acquisition of appropriate knowledge regarding computers.

3. Statistics has become more and more "complicated" due to the increasingly complex data structures and the more complex statistical methods and models. This requires an increasingly differentiated, yet specialized knowledge, which must, of course, also be part of the teaching process.

4. Regardless of the requirements of issues 1 through 3 , the time available for introductory courses has remained the same, or has even been reduced over the years.

In order to ensure a future statistical education that is still sufficient, it is necessary to adapt current teaching methodologies to newly developed methods, and organize them in an up-to-date manner. We do not suggest replacing blackboards, overhead transparencies or textbooks. They will surely remain essential tools for teaching statistics. However, additional interactive teaching tools can help to achieve the goals enumerated above. The challenge that accompanies the new multimedia technologies consists of supplementing the traditional teaching methodologies with these new technologies.

The structure of statistics lectures usually contain three central dimensions [RMZ00]: 


\subsection{Characteristics of $\mathrm{MM}^{*} \mathrm{Stat}$}

- First Dimension - Demonstration and explanation of the most important statistical methods and models, along with their assumptions (story line).

- Second Dimension - Reinforcement of learned content by offering background information and demonstrations of areas of applications, using elementary and complex examples.

- Third Dimension - Linking contents of the story line with each other.

$\mathrm{MM}^{*}$ Stat combines these three dimensions in a Web browser based tool. It can be used within the lecture, as well as for the independent reworking of the statistical content which students have learned in class. MM*Stat, therefore, works as a supplement, and not as a substitute, for traditional lectures.

For the production of teach-ware packages such as MM*Stat, the software tool MD*Book has been developed (see section 6.3). This tool allows for the creation of teaching materials in different variants - as introductory text containing detailed examples, as summary for repetition, as entry in a glossary, or as slides for a presentation within a lecture. Generated output can be of different types - printed on paper, as text on a screen for recalling and/or looking for certain content, or as text on a screen including interactive examples. Due to these features MM*Stat, could also be used for reengineering processes in enterprises.

\subsection{Characteristics of $\mathrm{MM}^{*}$ Stat}

The general design of $\mathrm{MM}^{*}$ Stat is based on the natural succession of statistics lectures [DHR99] - lecture units, explanation of statistical methods and models, additional background information and examples, and the revision of statistical methods already introduced in previous lectures (see figure 7.1).

The principle structure of $\mathrm{MM}^{*}$ Stat parallels a filing card system, allowing for rapid access of the entries - up to 10 "filing cards" can be opened at the same time. $\mathrm{MM}^{*}$ Stat consists of the main components:

- Lecture units,

- Additional information, 


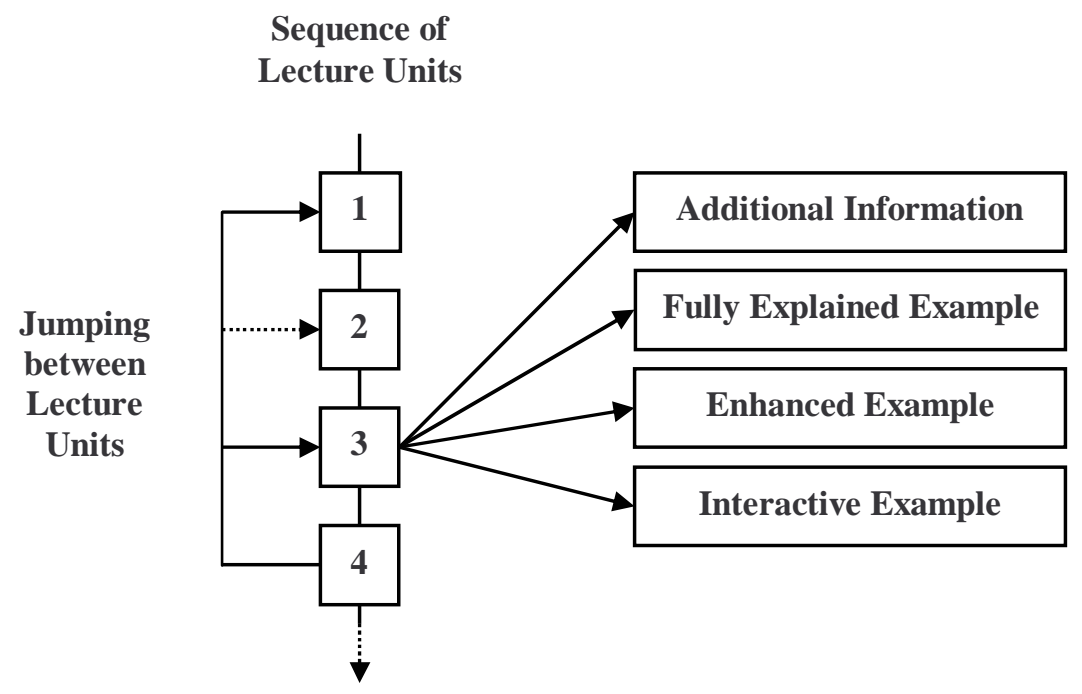

Figure 7.1: Three dimensions of teaching statistics

- Examples,

- Multiple choice questions.

\subsection{Lecture Units}

After opening the electronic statistics course, the first filing card appears on the screen showing a table of contents (figure 7.2). Here the user can select the desired lecture unit. Since $M^{*}$ Stat is meant to be a learning and a didactic program for a statistics introductory course, it contains the common and well-known topics: basic concepts of statistics, one-dimensional frequency distributions, basics of probability calculus, combinatorics, random variables, probability distributions, estimation methods, hypothesis testing, two dimensional distribution, regression analysis and time series analysis.

Each filing card provides an explanation of basic statistical methods and models, according to its lecture unit. It consists of the basic concept, definitions, formulas, graphics and fundamental statistical requirements of its application, but without any specific target area of application ("First Dimension"). Forward and Backward buttons enable the user to move along the sequence of processed lecture units, e.g. switching back in order to review certain content of a previous lecture unit (see figure 7.3). 


\subsection{Additional Information}

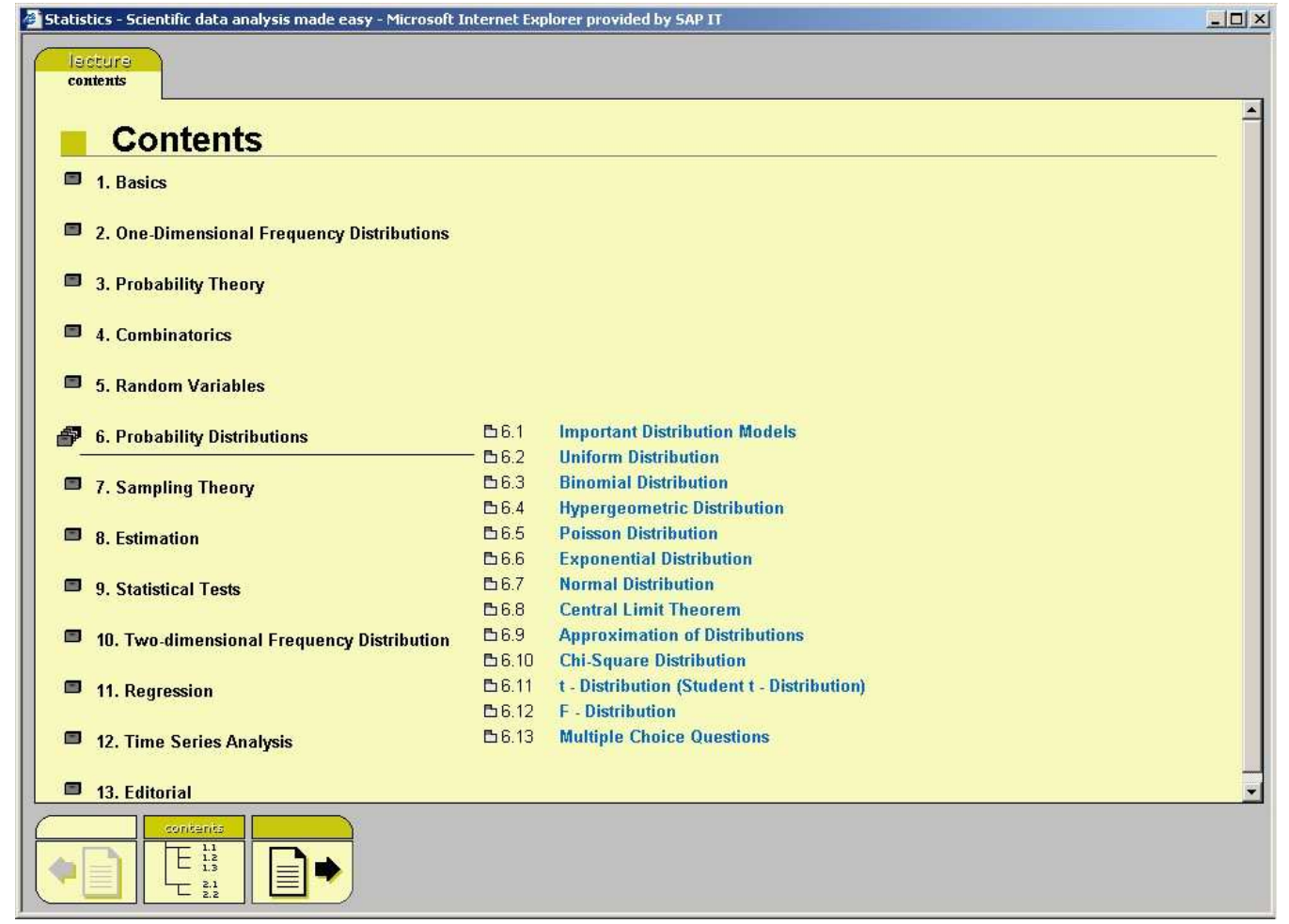

Figure 7.2: MM*Stat - Table of content

\subsection{Additional Information}

If required, additional information on the statistical methods and models explained in a lecture unit are offered. In this case, an Information icon appears on the lower right part of the screen. Additional information should deepen the understanding of statistical methods ("Second Dimension"). For example, the derivation of a method or special formula, or to offer additional textual explanations or comments on the main applications. Since the additional information appears on a separate filing card, the user is able to easily switch between Lecture card and Information card at any time.

\subsection{Examples}

Examples are essential for better understanding of the statistical knowledge imparted in the lecture units ("Second Dimension"). Therefore great at- 


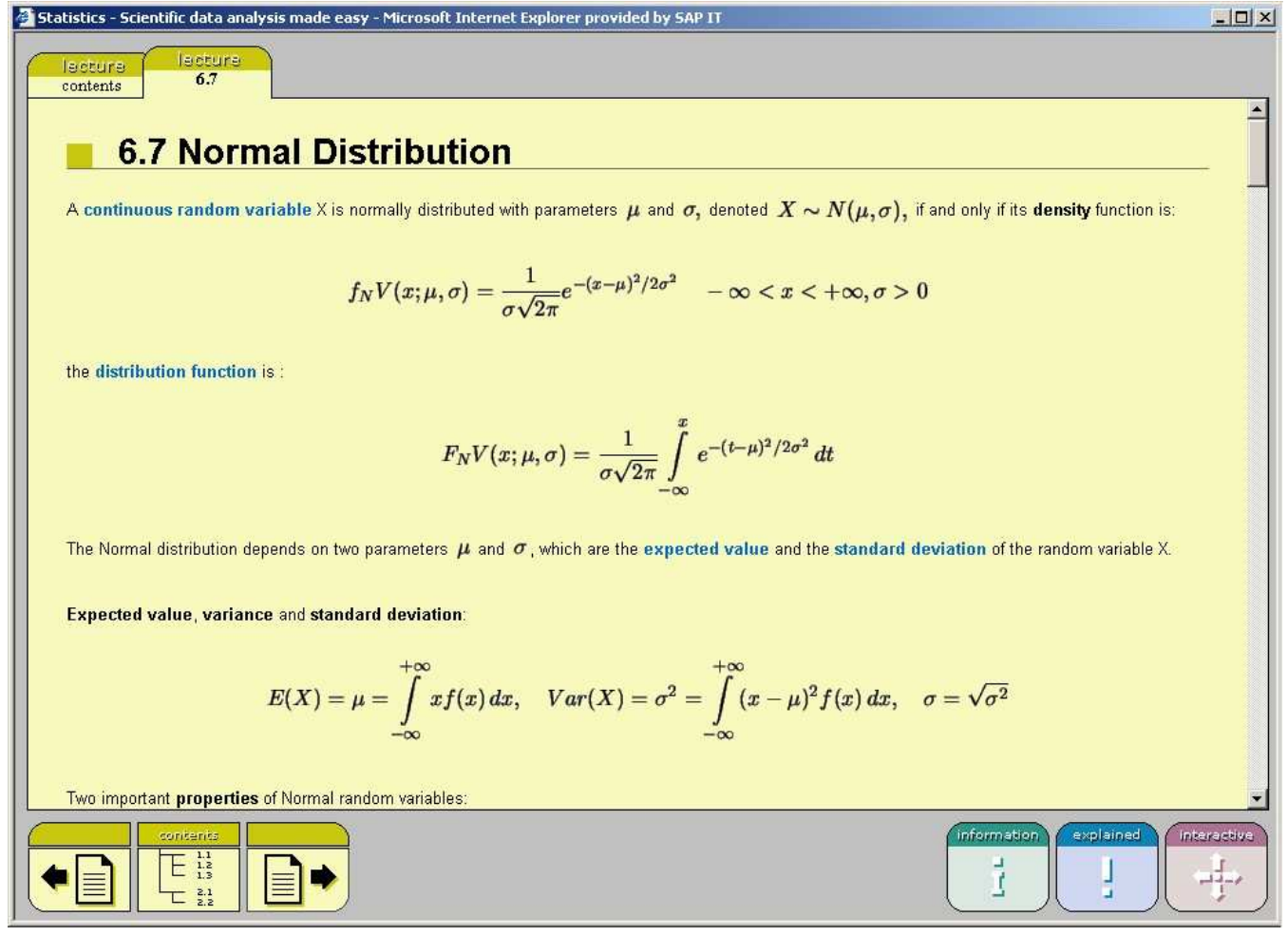

Figure 7.3: Lecture unit

tention was paid to examples while designing $\mathrm{MM}^{*}$ Stat. In general three different types of examples are offered by $\mathrm{MM}^{*}$ Stat:

- Fully explained examples,

- Enhanced examples,

- Interactive examples.

Not every type of example is offered for each of the lecture units. Buttons on the lower right corner of the screen indicate what examples are available for the selected lecture unit. Similar to the additional information, examples appear on a separate filing card. The user is therefore able to switch between Lecture card, Information card and Example card at any time. This represents the great advantage of a filing card system. A superscript tab indicates the filing card that is currently active. 


\section{Fully Explained Examples}

Fully explained examples are directly related to the content of the lecture unit from which they were called. As the name implies, this kind of example contains a complete explanation of the statistical problem. It begins with a description of the problem to be solved, followed by an illustration of data used, and continues with an exact determination of a method to be applied and the relevant formulas. Fully explained examples also demonstrate the processed calculations, and finally, give an interpretation of the calculated results.

\section{Enhanced Examples}

All the characteristics described above for the fully explained examples also apply for the enhanced examples. But this type of example goes one step further by offering at least one of the following features:

- They are related not only to the lecture unit from which they have been initiated, but also to previous lecture units.

- They compare various versions of a statistical method. For example, hypothesis testing based on a small sample and on a large sample.

- They comprise various statistical methods. For example, range, quartiles, mean, standard deviation, histogram and boxplot for a certain one-dimensional frequency distributions used within this example.

- They apply the same statistical method on different variables or data sets.

\section{Interactive Examples}

The most important characteristic of $\mathrm{MM}^{*}$ Stat is its interactive capability. This capability is embodied in the interactive examples. This kind of example takes into account the fact that "learning by doing" has prove to be the most effective. Within a practical teaching scenario this ideal is difficult to achieve, given the large number of students that take part in an introductory 
Interactive Teaching - MM*Stat

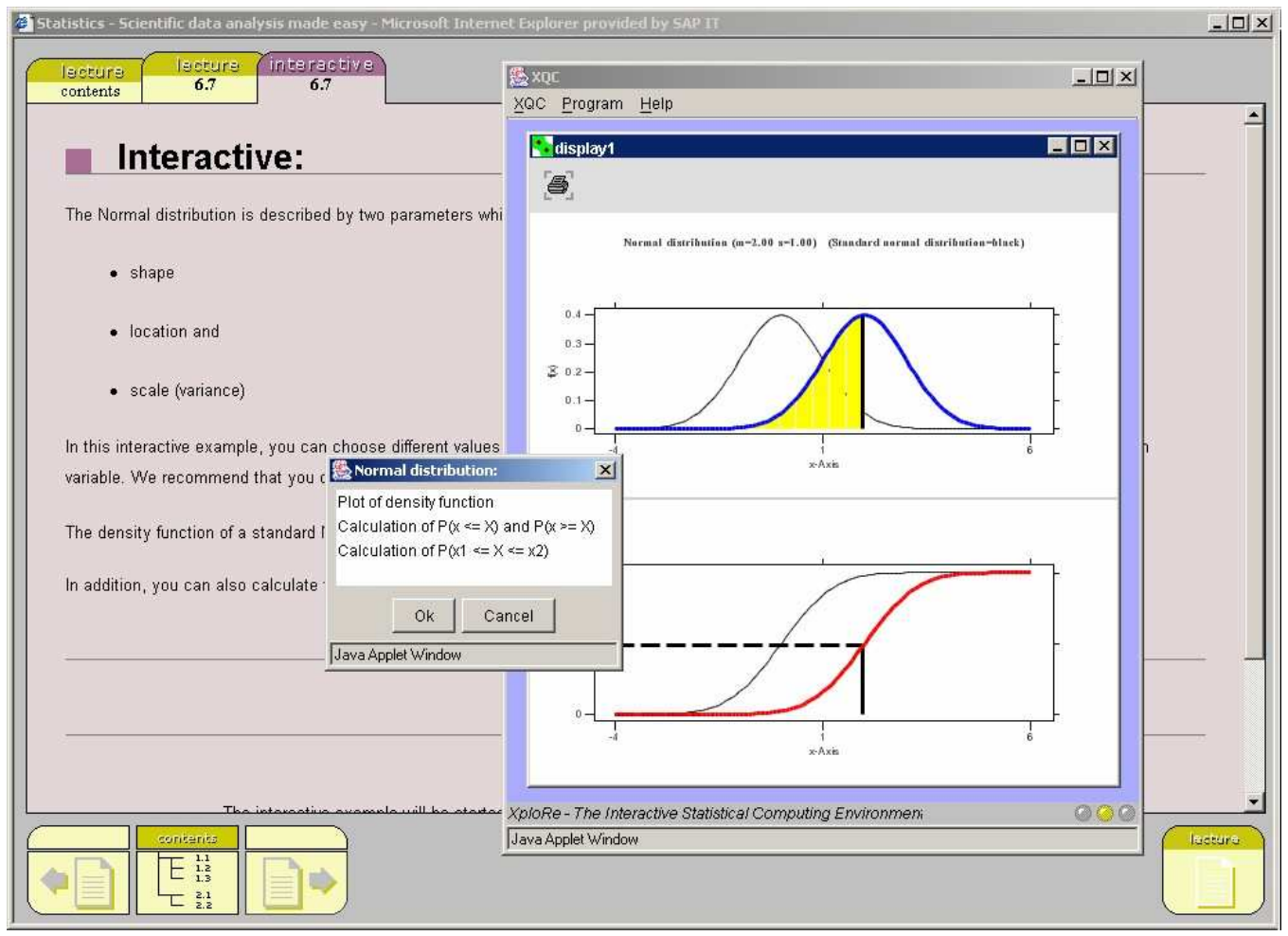

Figure 7.4: Interactive example

course, and the lack of time. MM*Stat can help to overcome those problems. The integration of the XploRe Quantlet Client/Server technology into $\mathrm{MM}^{*}$ Stat makes interactivity possible (see section 7.8 ). Interactive examples allow the user to practice repeatedly with various variables or data sets, and with alternate sample sizes or parameters, of the statistical methods applied. They ensure that the student can actively participate in the learning process, enabling him/her to study the effects of "playing" with the statistical method. In this manner, the student obtains a better understanding of how the statistical method works.

Figure 7.4 shows a running, interactive example for the lecture unit probability distributions ... normal distribution. In this interactive example, the user can choose different values for the two parameters expected value and standard deviation, and observe their effect on the density function of a normal random variable. The density function of a standard normal distribution is presented (in black) to provide a further reference point. In addition, the user can calculate the probability that $X$ falls in some interval. 


\subsection{Reinforcing Previously Learned Statisti- cal Content}

Working through the course, it would be helpful to be able to refer to concepts and methods, which had been introduced in previous lecture units ("Third Dimension"). Within an electronic course, the user must have the ability to jump back to a previously learned statistical aspect, without starting again from the very beginning of the course.

$\mathrm{MM}^{*}$ Stat offers different ways of referring to previous content. To recall a special term or definition, a glossary is available. Within a lecture unit, important terms are hyperlinked to this glossary. The glossary contains a short explanation of the linked term. Within the glossary, the term itself is also hyperlinked to the lecture unit in which it is explained in more detail.

A special filing card, Bookmarks, facilitates continuous work within the statistics course. It contains a list of previous filing cards (lecture units, information, examples etc.) that have been opened, and allows for jumping directly into one of these lecture units or examples.

\subsection{Additional Features of MM*Stat}

At the end of each topic, MM*Stat offers multiple choice questions. These questions enable the student to check his/her knowledge. Feedback showing the results of the answers can be shown on demand. The student can choose from two different types of feedback: evaluation without indicating errors, or evaluation indicating errors. Alternatively, the student can force MM*Stat to check all correct answers.

Some lecture units offer multimedia content, such as audio files. These files are used for explaining statistical facts or illustrating examples.

MM*Stat also offers a help system that can be accessed via the table of contents filing card. This help system contains technical help (e.g. verification of whether the computer and browser being used are suitable for MM*Stat), as well as help for using $\mathrm{MM}^{*}$ Stat (e.g. how to open and close filing cards, etc.). 


\subsection{Technical Parameters of $\mathrm{MM}^{*}$ Stat}

$\mathrm{MM}^{*}$ Stat is based on HTML 4.0, combined with JavaScript and Cascading Style Sheets (CSS) techniques. HTML is used for text shown on filing cards and to realize the hyperlink functionality. Dynamic CSS-documents determine the internal structure of $\mathrm{MM}^{*}$ Stat filing cards, such as background design, typographic attributes of filing cards and hyperlinks. JavaScript programs (ECMA script) allow for comfortable and innovative navigation through all lecture units and examples within $\mathrm{MM}^{*}$ Stat. Formulas and graphics are integrated using graphical standards such as GIF or JPEG format.

The technical minimum requirements for an optimal use of MM*Stat are:

- Pentium II, $200 \mathrm{MHz}$ or similar,

- CD-ROM drive (for using the CD-version of $\mathrm{MM}^{*}$ Stat),

- Internet connection (for using the online version of MM*Stat),

- Microsoft Internet Explorer 5.0 or higher,

- JavaScript 1.1/1.2 or JScript,

- Java Runtime Environment 1.3,

- Sound and video capabilities.

As already mentioned, one of the special features $M^{*}$ Stat offers is its interactive capability, achieved by the integration of the XploRe Quantlet Client/Server architecture (see section 3). It enables the user to study statistical methods with varying application conditions, or by using different data sets without the need of installing additional (statistical) software.

The graphical user interface (GUI) is provided by the XploRe Quantlet Client (XQC). The XploRe server used by the client is running either locally $\left(\mathrm{MM}^{*}\right.$ Stat on CD-ROM) or on a remote computer (MM*Stat online). Due to the potential for starting the $\mathrm{XQC}$ as a Java applet, the integration process into $\mathrm{MM}^{*}$ Stat is quite simple. The required HTML code (see section 4.1.1) can be generated automatically by using MD*Book (see section 6.3). Figure 7.4 illustrates a running interactive example. It shows the XQC, 


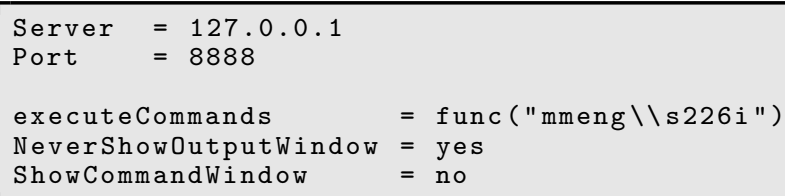

Figure 7.5: s226i.ini

which has been initiated as part of this interactive example. In order to meet the requirement of the underlying (interactive) example, the XQC has to be configured accordingly. For this purpose, the xqc.ini file must be adjusted. Figure 7.5 shows the configuration file used for this example. Upon starting the interactive example, the XQC attempts to connect to the stated server and port. It starts without showing output window and console - in a "Golden Solution" mode. The Statement

executeCommands $=$ func $($ "mmeng \\s226i")

causes the XQC to activate the required XploRe Quantlet s226i.xpl immediately. This XploRe Quantlet plots the density function of a normal distribution and allows the changing of parameters via the XploRe components select item and read value. To the user, the XQC behaves as a common Java applet which has been programmed just for this example.

Interactivity within MM*Stat is not "limited" to merely executing XploRe Quantlets. Using the features offered by the XQC allows for integration of examples where the user is able to access the underlying Quantlet. Thus, an experienced user could edit the code, adjust it to his/her needs, and execute it again.

\subsection{User Acceptance}

$\mathrm{MM}^{*}$ Stat has been practically used since 1999, as an additional tool within a statistics introductory course, and not only used as part of the lecture. Students can also buy the MM*Stat teach-ware package on CD-ROM. This enables them to reinforce content learned in class independently. An inquiry of students attending the class during 1999 revealed that 72 percent already work with this tool. 75 percent of the students expressed that MM*Stat helped them to better understand the statistical content. 


\subsection{Related Projects}

Based on the knowledge gained with $\mathrm{MM}^{*}$ Stat, other teach-ware packages have been developed recently, or are currently in development.

A current example results from a co-operation agreement between the University of Toronto and the Center for Applied Statistic and Economics Institute for Statistics and Econometrics (CASE). Both institutions have started a project with the subject "A Web Based Statistics Course for Economics Undergraduates". The aim of this project is to develop a teach-ware package that supports the teaching of basic statistics at the University of Toronto. Our XploRe Quantlet Client/Server model provides one major portion of this package by enriching it with net based, interactive contents.

Interactive teaching is by no means limited only to use within basic statistic courses. The "Finance Introductory Course" (FIC) represents only one example of using interactive techniques in advanced statistics courses. This course offers a complete introduction into financial statistics containing topics such as Option Management, Black Scholes Option Pricing Model, ARIMA Models and $A R C H / G A R C H$ Models.

A list of projects, as well as further information on interactive teaching, is available at http://www.md-stat.com. 


\section{Chapter 8}

\section{Conclusions}

In this thesis, we described a client/server approach for statistical computing. Our approach combines the possibilities of a powerful statistical software environment, with the advantages of distributed applications, and the opportunities offered by the Internet. The result is a statistical package - usable via the World Wide Web - that behaves like a traditional statistical software package, without the need for installing the entire software package. Nevertheless, it should not be seen as a substitute for "traditional" statistical software packages but as an additional tool for statistical computing.

Our architecture offers access to a powerful computing engine that is based on the statistical computing environment XploRe. A middleware - represented by $\mathrm{MD}^{*}$ Serv - handles server side communications. The communication between client and server relies on TCP/IP working as the transportation protocol. For client side communication, we use a protocol - MD*Crypt - that has been developed for interaction with a statistical environment. With MD*Crypt, a package is provided to deploy statistical methods and computing power that help to solve statistical problems. If programming skills are assumed, it is highly adjustable to a wide range of environments. The XploRe Quantlet Client (XQC) represents the front end of our architecture. It takes advantage of objects and methods provided by the MD*Crypt package to present server results to the user. The XQC is realized in pure Java. Running the XQC as a Java applet, it becomes available on almost any machine connected to the World Wide Web.

Following W3C's definition of a Web Service (see section 2.3), our client/server approach does not meet the required characteristics to call itself a Web Service. We use neither the Web Service Description Language 


\section{Conclusions}

(WSDL) nor XML, for communication between client and server. Instead, we have defined our own (open) protocol in order to limit the amount of data that has to be transferred. Despite this difference, our client/server approach works similarly to what is defined as being a Web Service - a client that communicates with a server via the Internet using a standardized open protocol.

As shown in Chapters 6 and 7, our XploRe Quantlet Client/Server architecture has already been successfully used in real life applications. Integrating the XQC into electronic publications allows for regeneration and reproduction of statistical results presented within the publication. Being part of the teach-ware package $\mathrm{MM}^{*}$ Stat, the XQC/XQS architecture allows for interactivity, supporting teaching and learning statistics in introductory courses.

Our client/server architecture also has its limitations and drawbacks. XQC, as well as $\mathrm{MD}^{*}$ Crypt, rely on a Java Runtime Environment. In order to access the XploRe Quantlet Server, the user might have to install additional software on the client's computer. Another drawback is related to Java's applet technology. Since applets are regarded as potential security problems, some crucial functionality is not allowed by them. Examples of these restrictions are the disabled copy/paste functionality, and the impossibility of accessing local files. Running the XQC as a signed applet would help to overcome these restrictions.

Another well-known problem is the security of data exchanged via the Internet. Data encoding would be a reasonable solution to this security problem, however, this would slow down the communication process.

The facts mentioned above imply that our XploRe Quantlet Client/Server architecture still has some challenges that need to be resolved. 


\section{Bibliography}

[AHKS01] G. Aydinli, W. Härdle, T. Kleinow, and H. Sofyan. Rex: Modern statistical tools in office applications. Proc. of the ISM symposium "Statistical software in the Internet age", pages 101-109, 2001. Institute of Statistical Mathematics, Tokyo.

[BD95] J. Buckheit and D. Donoho. in Antoniadis, A. (ed.): WaveLab and Reproducible Research, chapter Wavelets and Statistics. Springer Verlag, New York, 1995.

[Bla00] A. Blauth. GraphFitI - A program for model selection in graphical chain models. available online at http://www.stat.unimuenchen.de/ `blauth/GraphFitI/graphFitI.html, 2000. last access: March 2004.

[BN84] A. Birrel and B. Nelson. Implementing remote procedure calls. ACM Transactions on Computer Systems, 2(1), 1984.

[Bur99] M. Burkitt. White paper: Thin client/server computing lets you take control, IT Management: Enterprise \& Supply Chain. ComputerWeekly.com, available online at http://www.computerweekly.com/Article42065.htm, 1999. last access: March 2004.

[CKS00] J. Claerbout, N. Karrenbach, and M. Schwab. Making scientific computations reproducible. Computing in Science $\mathscr{E}$ Engineering, 2(6):61-67, Nov.-Dec. 2000. available online at http://sep.stanford.edu/research/redoc/cip.html.

[Cla02] J. Claerbout. Making Research Reproducible. available online at http://sepwww.stanford.edu/research/redoc/IRIS.html, 2002. last access: March 2004. 


\section{BIBLIOGRAPHY}

[Deg02] A. Degenring. Diploma Thesis "Konzeption und Implementierung zur Integration eines SAS Servers in SOAPbasierte Web Service Umgebungen". available online at http://www.degenring.de/webservices/diplomarbeit.html, 2002. last access: March 2004.

[DHR99] N. Derby, W. Härdle, and B. Rönz. The Three Dimensions of Multimedia Teaching of Statistics. Discussion Paper No. 76, Sonderforschungsbereich 373, 1999. Humboldt-Universität zu Berlin.

[dJ02] I. de Jong. Web Services/SOAP and CORBA. available online at http://www.arbores.ca/bryan/papers/corbaVsSoap/index.html, 2002. last access: March 2004.

[Feu01] J. Feuerhake. MD*CRYPT - the XQS/XQC protocol. available online at http://www.md-crypt.com, 2001. last access: March 2004.

[Gen03] J. Gentle. Computational Statistics. available online at http://www.galaxy.gmu.edu/ ${ }^{\sim}$ jgentle/cmpstbk/, 2003. last access: March 2004.

[GMK ${ }^{+}$97] O. Günther, R. Müller, R. Krishnan, H. Bhargava, and P. Schmidt. MMM: A Web-Based System for Sharing Statistical Computing Modules. IEEE Internet Computing, 1(3):59-68, 1997.

[GTHM01] O. Günther, G. Tamm, L. Hansen, and T. Meseg. Application Service Providers: Angebot, Nachfrage und langfristige Perspektiven. Wirtschaftsinformatik, 43(6):555-567, 2001.

[GTL03] R. Gentleman and D. Temple Lang. Statistical Analysis and Reproducible Research. available online at http://www.biostat.harvard.edu/ ${ }^{\sim}$ rgentlem/Pdf/RR.pdf, 2003. last access: March 2004.

[HKM99] W. Härdle, S. Klinke, and M. Müller. XploRe - The Statistical Computing Environment. Springer-Verlag, New York, 1999.

[HKT01] W. Härdle, T. Kleinow, and R. Tschernig. Web quantlets for time series analysis. Annals of the Institute of Statistical Mathematics, 53(1):179-188, 2001. 
[HR02] W. Härdle and B. Rönz. E-Learning/E-Teaching of Statistics: Students and Teachers Views. In J.J. Lee, editor, The 4th conference of the Asian Regional Section of the IASC "e-statistics for information society", pages 222-227, December 5-7 2002.

[IAYY01] T. Inoue, Y. Asahi, Y. Yamamoto, and H. Yadohisa. A prototype of Data Representation System. Proc. of the ISM symposium "Statistical software in the Internet age", pages 85-90, 2001. Institute of Statistical Mathematics, Tokyo.

[KFYN01] I. Kobayashi, T. Fujiwara, Y. Yamamoto, and J. Nakano. The Language and the Extendibility of the Statistical System Jasp. Proc. of the ISM symposium "Statistical software in the Internet age", pages 65-73, 2001. Institute of Statistical Mathematics, Tokyo.

[KL01] T. Kleinow and H. Lehmann. Client/Server Based Statistical Computing. Proc. of the ISM symposium "Statistical software in the Internet age", pages 1-8, 2001. Institute of Statistical Mathematics, Tokyo.

[Kli01] S. Klinke. MD*book - a tool for creating interactive documents. Proc. of the ISM symposium "Statistical software in the Internet age", pages 75-84, 2001. Institute of Statistical Mathematics, Tokyo.

[KT00] T. Kleinow and M. Thomas. in Franke, J. and Härdle, W. and Stahl, G. (eds.): Measuring Risk in Complex Stochastic Systems in Lecture Notes in Statistics, chapter Computational resources for extremes. Springer-Verlag, New York, 2000.

[Lan99] D. M. Lane. The Rice Virtual Lab in Statistics. Behavior Research Methods, Instruments \&G Computers, 31(1):24-33, 1999.

[Lei02] F. Leisch. Sweave: Dynamic generation of statistical reports using literate data analysis. Proc. in Computational Statistics, pages 575-580, 2002.

[Lew98] S.M. Lewandowski. Frameworks For Component-Based Client/Server Computing. ACM Computing Survey (CSUR), 30(1):327, 1998. 
[Mau01] F. Maurer. in: Marciniak, J. J. (ed.): Software Engineering Encyclopedia, chapter Engineering Web Applications with Java. Wiley, December 2001.

[Mic96] Microsoft Corporation. DCOM: A Business Overview, MSDN Library. available online at http://msdn.microsoft.com/library/enus/dndcom/html/msdn_dcombiz.asp, 1996. last access: March 2004 .

[Mic97] Microsoft Corporation. DCOM Technical Overview, MSDN Library. online available at http://msdn.microsoft.com/library/enus/dndcom/html/msdn_dcomtec.asp, 1997. last access: March 2004.

[Mor04] Y. Mori. Information are available online at http://www.soci.ous.ac.jp/ ${ }^{\sim}$ mori/topE.htm, 2004. last access: March 2004.

[OHE96] R. Orfali, D. Harkey, and J. Edwards. The Essential Client/Server Survival Guide. John Wiley \& Sons, New York, 1996.

[RMZ00] B. Rönz, M. Müller, and U. Ziegenhagen. Compstat 2000: Proceedings in Computational Statistics, chapter The Multimedia Project MM*STAT for Teaching Statistics, pages 409-414. Physica-Verlag, Heidelberg, 2000.

[Sie00] J. Siegel. CORBA 3 Fundamentals and Programming. Wiley Press, New York, 2nd edition, 2000.

[Sko02] A. Skonnard. The Birth of Web Services. MSDN Magazin, October 2002 .

[SSJE02] I. Singh, B. Stearns, M. Johnson, and Enterprise Team. Designing Enterprise Applications with the J2EE Platform. AddisonWesley, New York, 2nd edition, 2002.

[Sun04a] Sun Microsystems, Inc. CORBA Technology and the Java 2 Platform, Standard Edition. available online at http://java.sun.com/j2ee/corba/index.html, 2004. last access: March 2004. 
[Sun04b] Sun Microsystems, Inc. Java Remote Method Invocation - Distributed Computing for Java, White Paper. available online at http://java.sun.com/products/jdk/rmi/reference/ whitepapers/javarmi.html, 2004. last access: March 2004.

[SV02] J. Symanzik and N. Vukasinovic. in in Härdle, W., Rönz, B. (eds.): Compstat 2002: Proceedings in Computational Statistics, chapter Teaching Statistics with Electronic Textbooks, pages 7990. Physica-Verlag, Heidelberg, 2002.

[Tem02] D. Temple Lang. Calling $\mathrm{R}$ from Java. available online at http://www.omegahat.org/RSJava/RFromJava.pdf, 2002. last access: March 2004.

[Tid00] D. Tidwell. Web services: the Web's next revolution, IBM Web Service Tutorial. available online at http://www.ibm.com/developerworks/views/webservices/ tutorials.jsp, 2000. last access: March 2004.

[Tod04] V. Todorov. WebJMCD: Web Services and Computing for Robust Statistics. In ICORS 2003: Proceedings of International Conference on Robust Statistics. University of Antwerp, Antwerp, Belgium, 2004. available online at http://www.win.ua.ac.be/ ${ }^{\sim}$ icors03/abstract/todorov.pdf, last access: March 2004.

[Vla02] I. Vlad. Reproducibility in computer-intensive sciences. Ad Astra 2002, 1(2), 2002. available online at http://www.adastra.ro/journal/2/vlad_reproducibility.pdf, last access: March 2004.

[W3C02] W3C. Web Service Glossary, Web Service Activity. available online at http://www.w3.org/2002/ws/, 2002. last access: March 2004.

[Wai02] P. Wainewright. Web Service Infrastructure. available online at http://www.philwainewright.com/pubs/wp/WSIpaper.pdf, 2002. last access: March 2004.

[WK02] R. Witzel and S. Klinke. MD*Book online \& e-stat: Generating e-stat Modules from LaTeX. Proc. in Computational Statistics, pages 449-454, 2002. 
[WWH04] R.W. West, Y. Wu, and D. Heydt. An Introduction to StatCrunch 3.0. Journal of Statistical Software, 9(5), 2004. available online at http://www.jstatsoft.org/v09/i05/scjss/, last access: March 2004. 


\section{Appendix A}

\section{License Agreement}

The XploRe Quantlet Client (XQC) can be used free of charge. It does not come with any support, and is offered to the public in the spirit of the University of Illinois/NCSA Open Source License:

Copyright (c) $2004 \mathrm{MD}^{*}$ Tech

All rights reserved.

Developed by: Heiko Lehmann, Prof. Dr. Wolfgang Härdle

MD*Tech - Method and Data Technologies

http://www.mdtech.de

Permission is hereby granted, free of charge, to any person obtaining a copy of this XploRe Quantlet Client (XQC) and associated documentation files (the "Software"), to deal with the Software without restriction, including without limitation the rights to use, copy, modify, merge, publish, distribute, sublicense, and/or sell copies of the Software, and to permit persons to whom the Software is furnished to do so, subject to the following conditions:

- Redistributions of source code must retain the above copyright notice, this list of conditions and the following disclaimers.

- Redistributions in binary form must reproduce the above copyright notice, this list of conditions and the following disclaimers in the documentation and/or other materials provided with the distribution. 


\section{License Agreement}

- Neither the names of Heiko Lehmann, Prof. Dr. Wolfgang Härdle, MD*Tech, nor the names of its contributors may be used to endorse or promote products derived from this Software without specific prior written permission.

THE SOFTWARE IS PROVIDED "AS IS", WITHOUT WARRANTY OF ANY KIND, EXPRESS OR IMPLIED, INCLUDING BUT NOT LIMITED TO THE WARRANTIES OF MERCHANTABILITY, FITNESS FOR A PARTICULAR PURPOSE AND NONINFRINGEMENT. IN NO EVENT SHALL THE CONTRIBUTORS OR COPYRIGHT HOLDERS BE LIABLE FOR ANY CLAIM, DAMAGES OR OTHER LIABILITY, WHETHER IN AN ACTION OF CONTRACT, TORT OR OTHERWISE, ARISING FROM, OUT OF OR IN CONNECTION WITH THE SOFTWARE OR THE USE OR OTHER DEALINGS WITH THE SOFTWARE.

(Source: http://www.opensource.org/licenses/UoI-NCSA.html) 


\section{Appendix B}

\section{XQC Source Code}

The following pages contain the source code of selected classes (discussed in this thesis) that are part of the XploRe Quantlet Client.

\section{B.1 XClient.java}

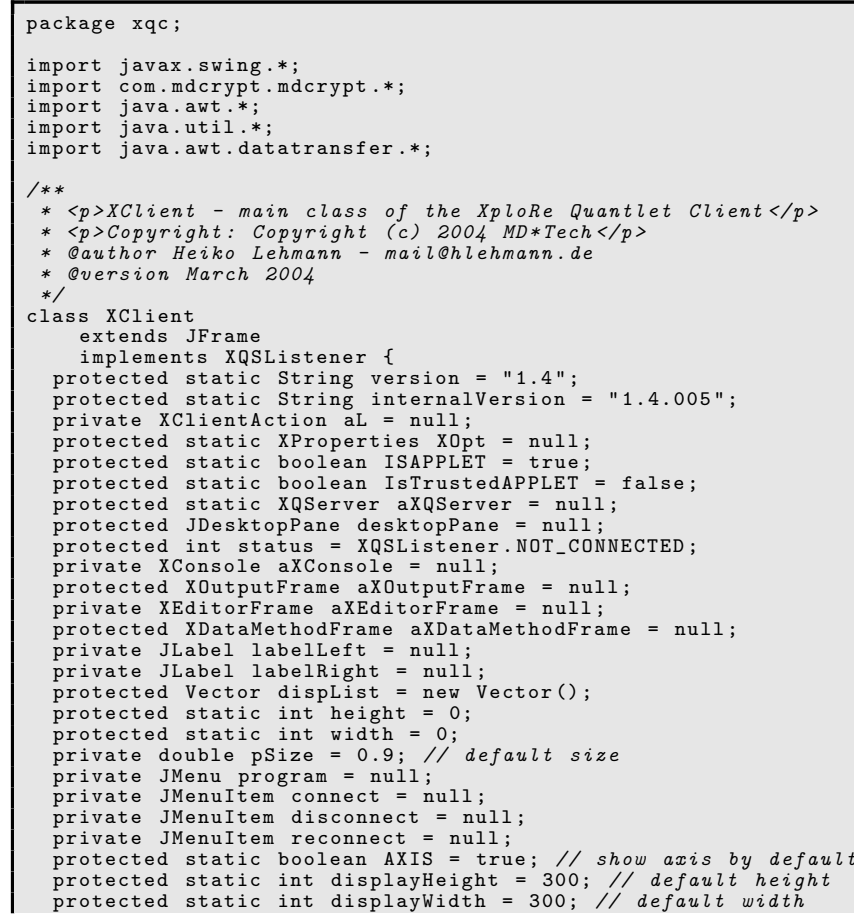




\section{XQC Source Code}

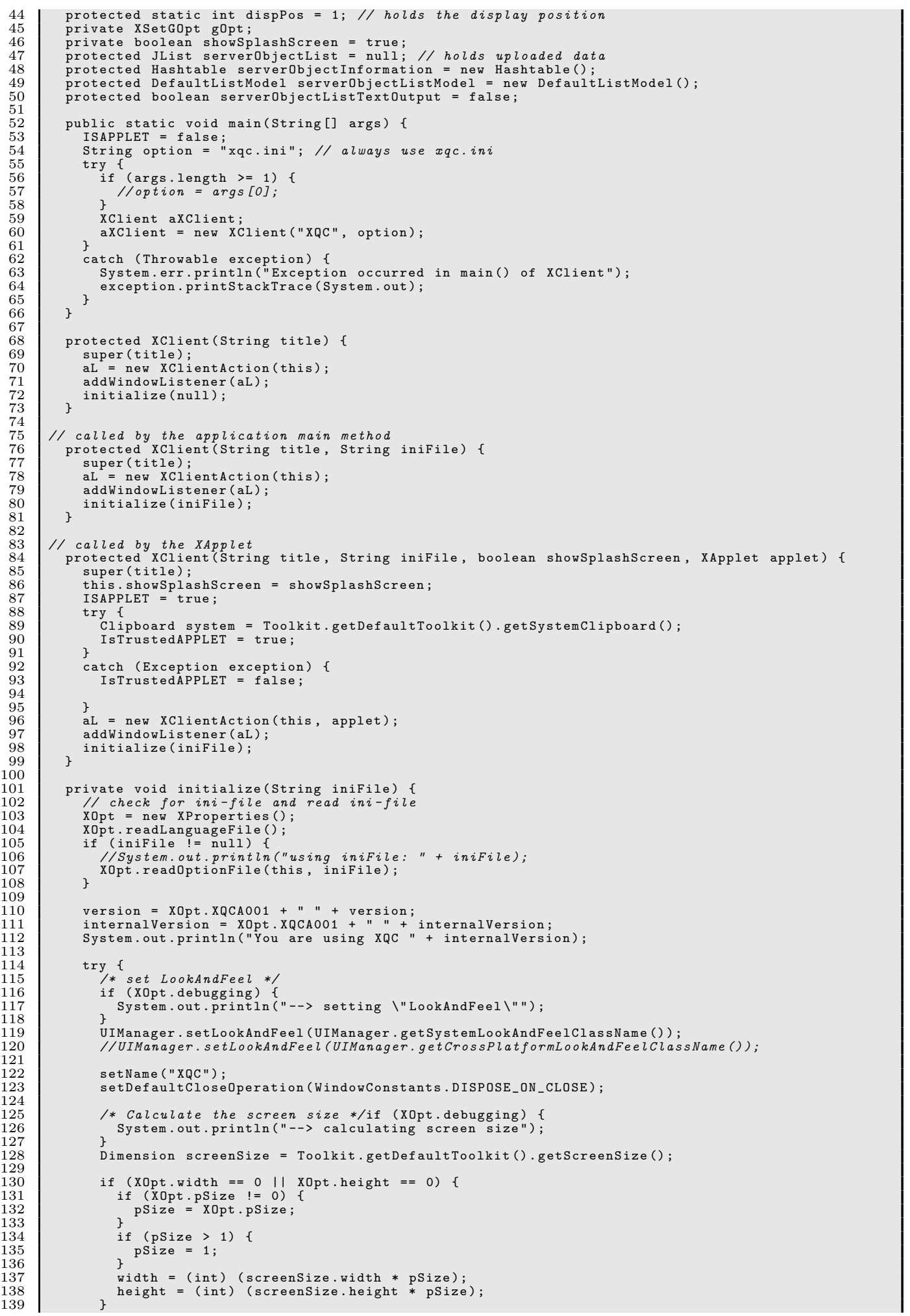




\section{B.1 XClient.java}

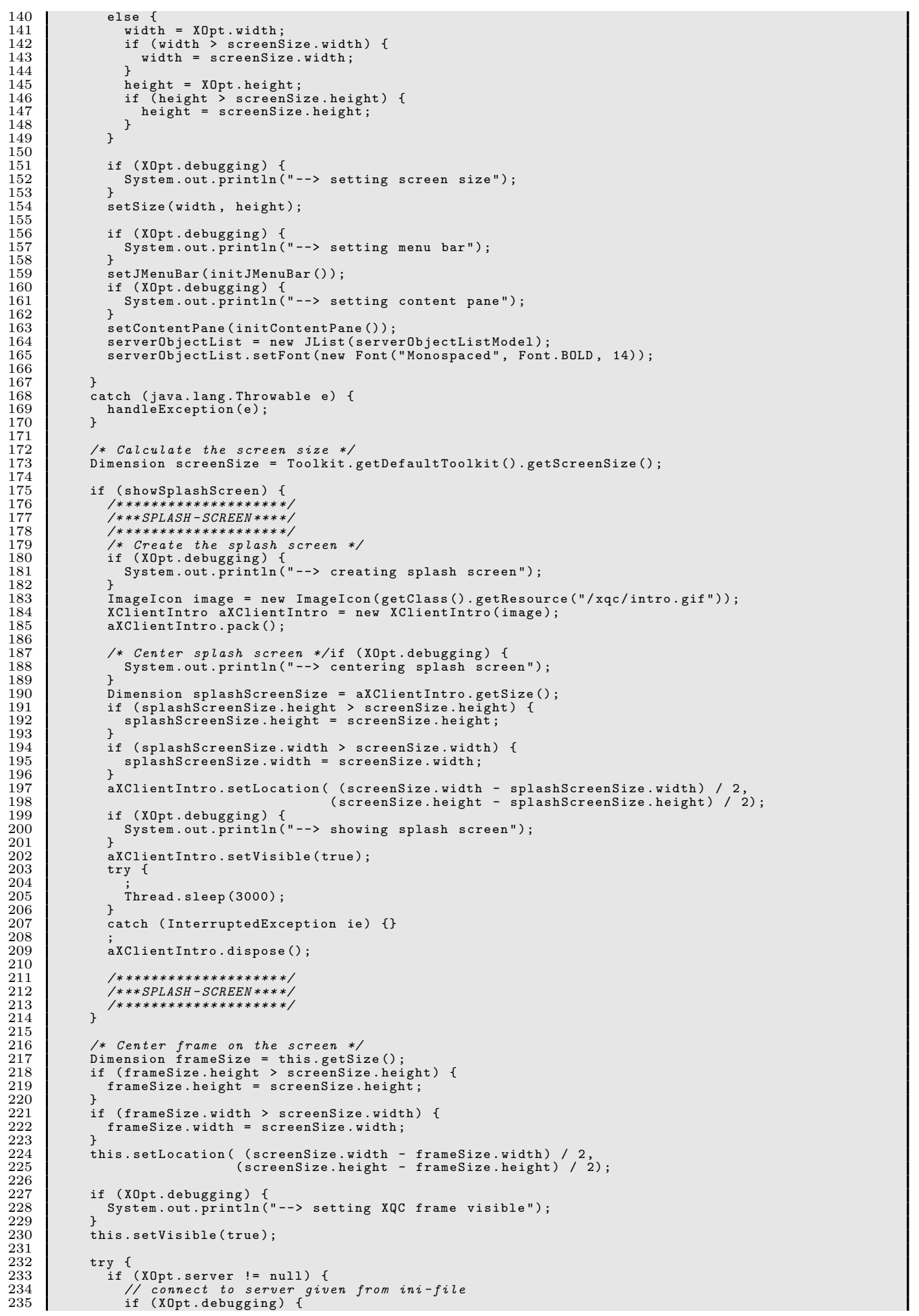




\section{XQC Source Code}

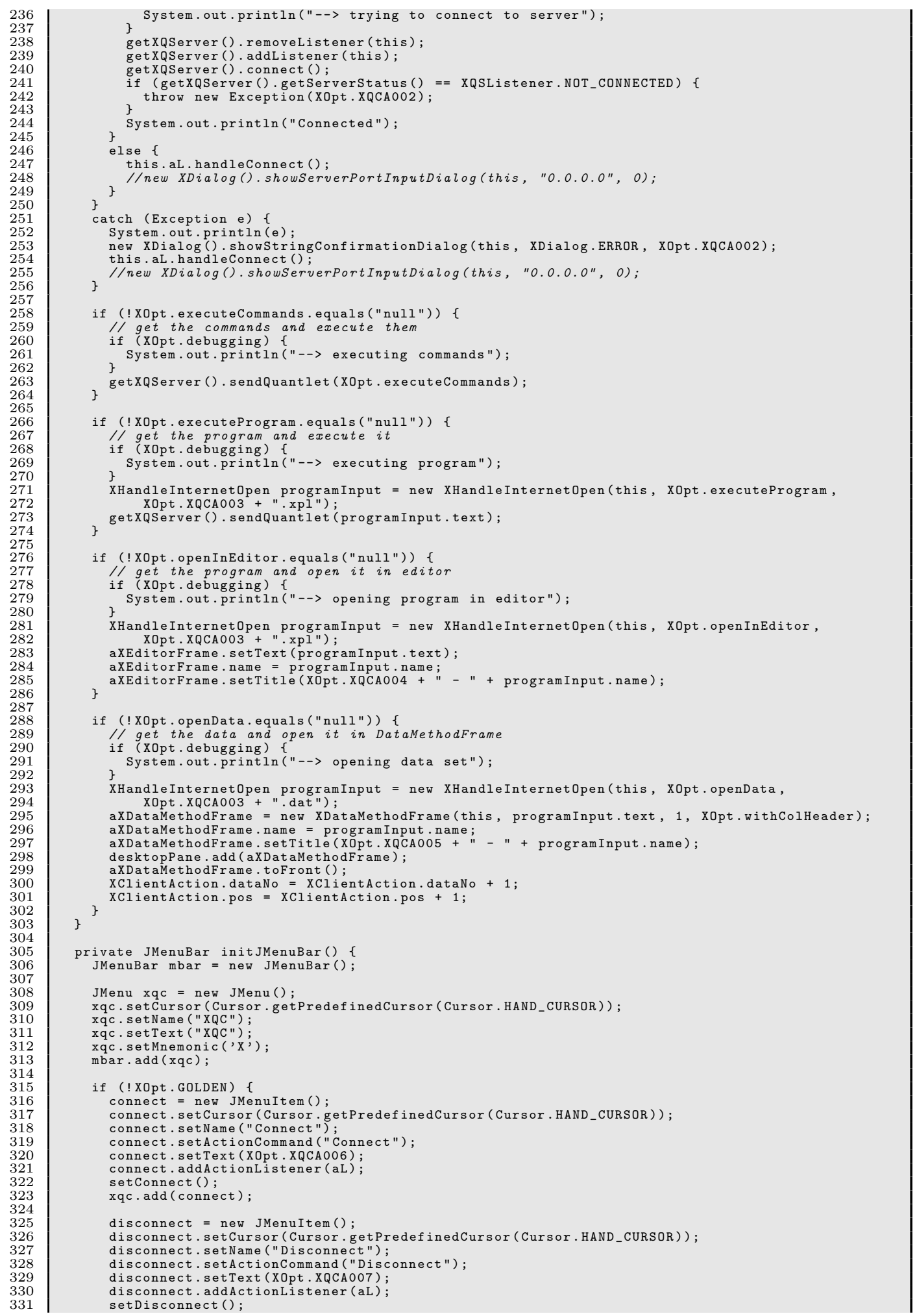




\section{B.1 XClient.java}

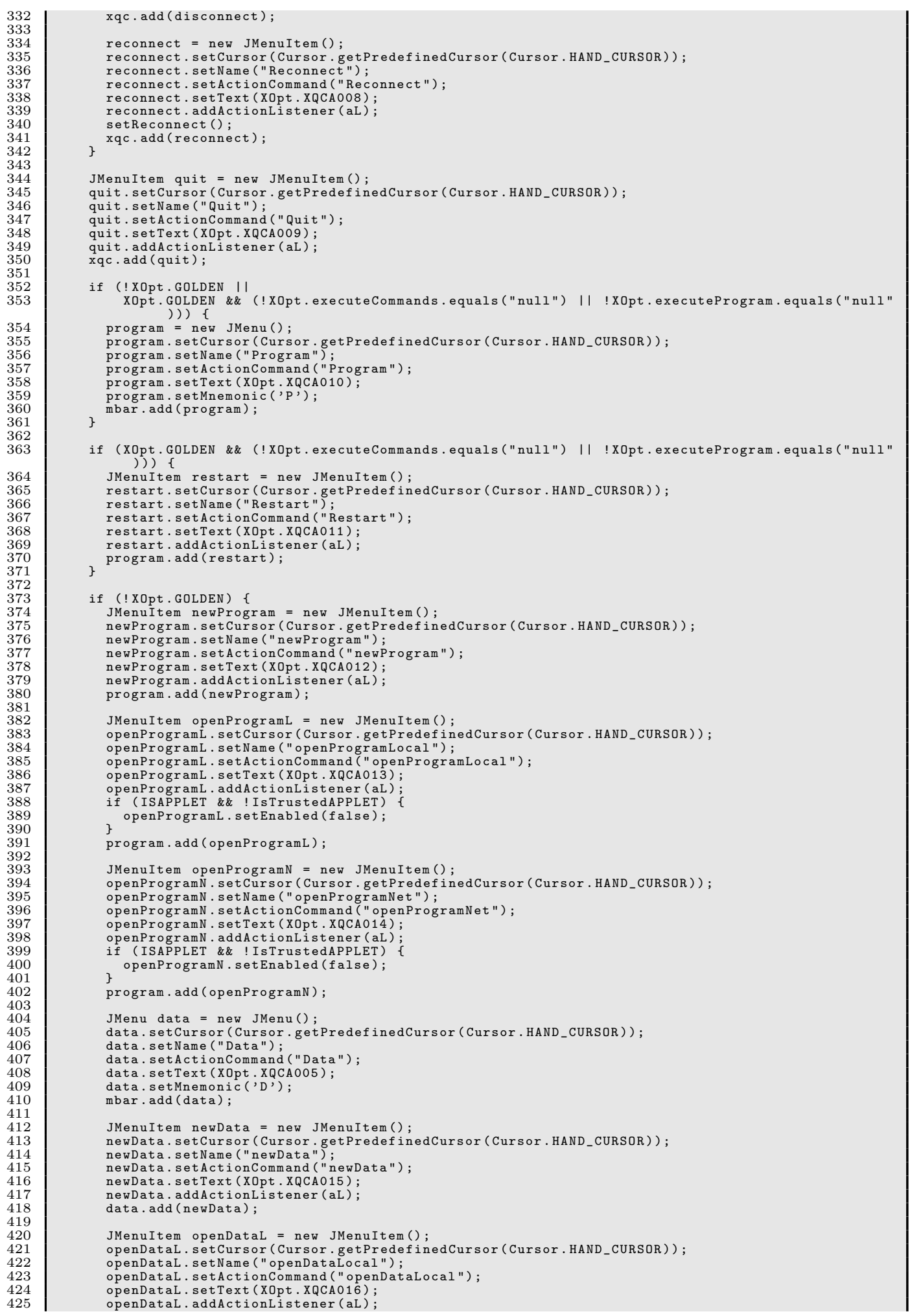




\section{XQC Source Code}

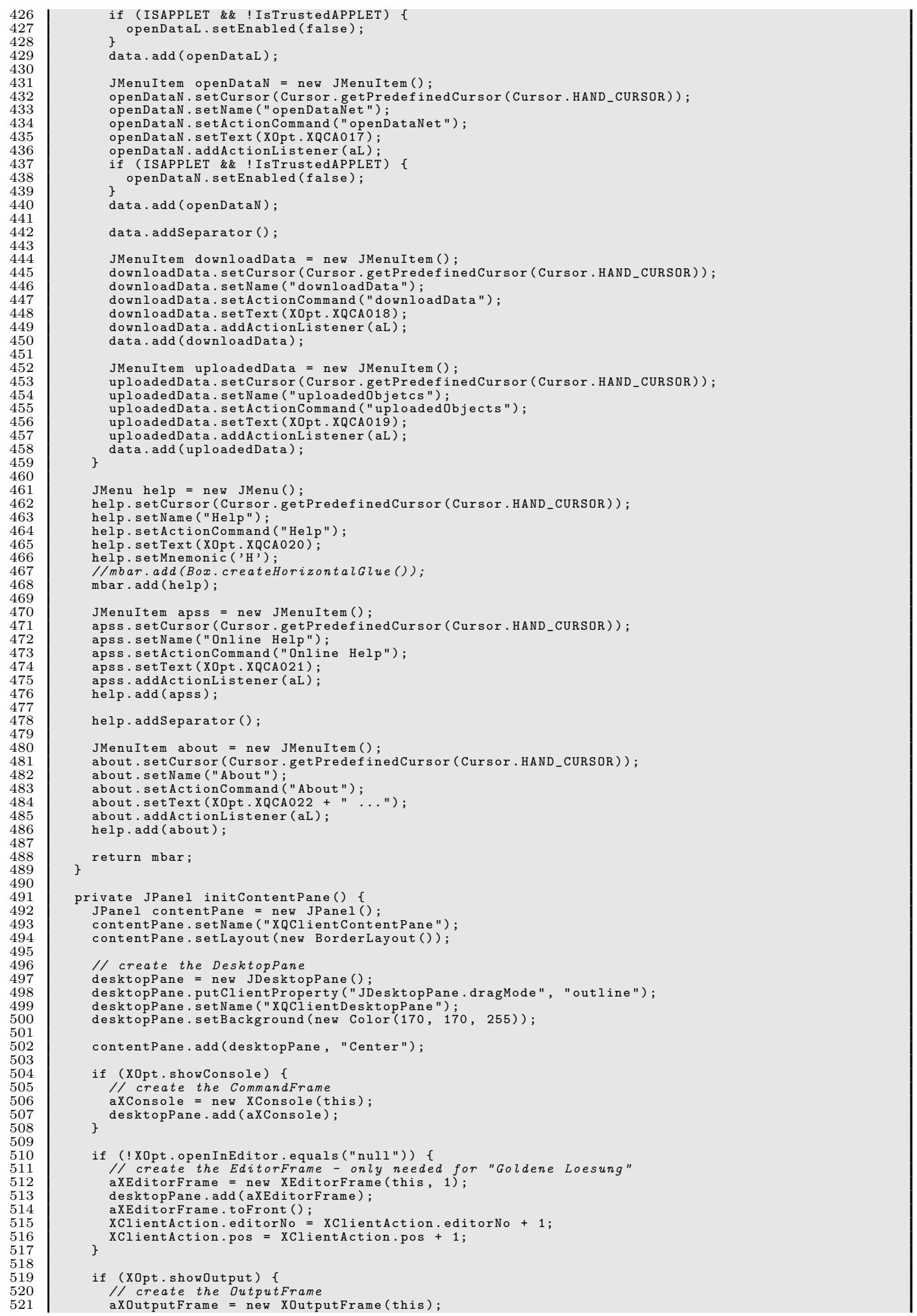




\section{B.1 XClient.java}

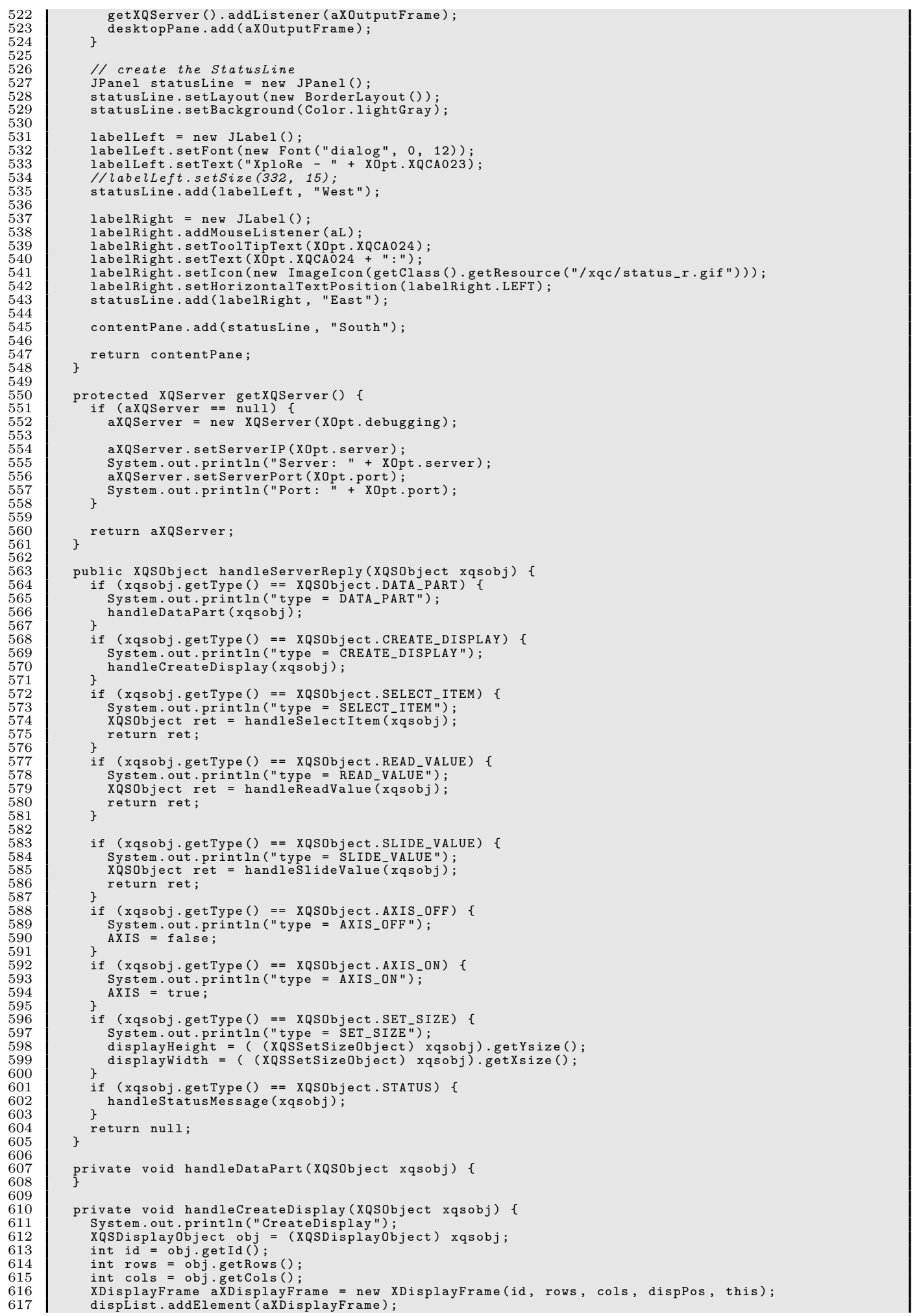




\section{XQC Source Code}

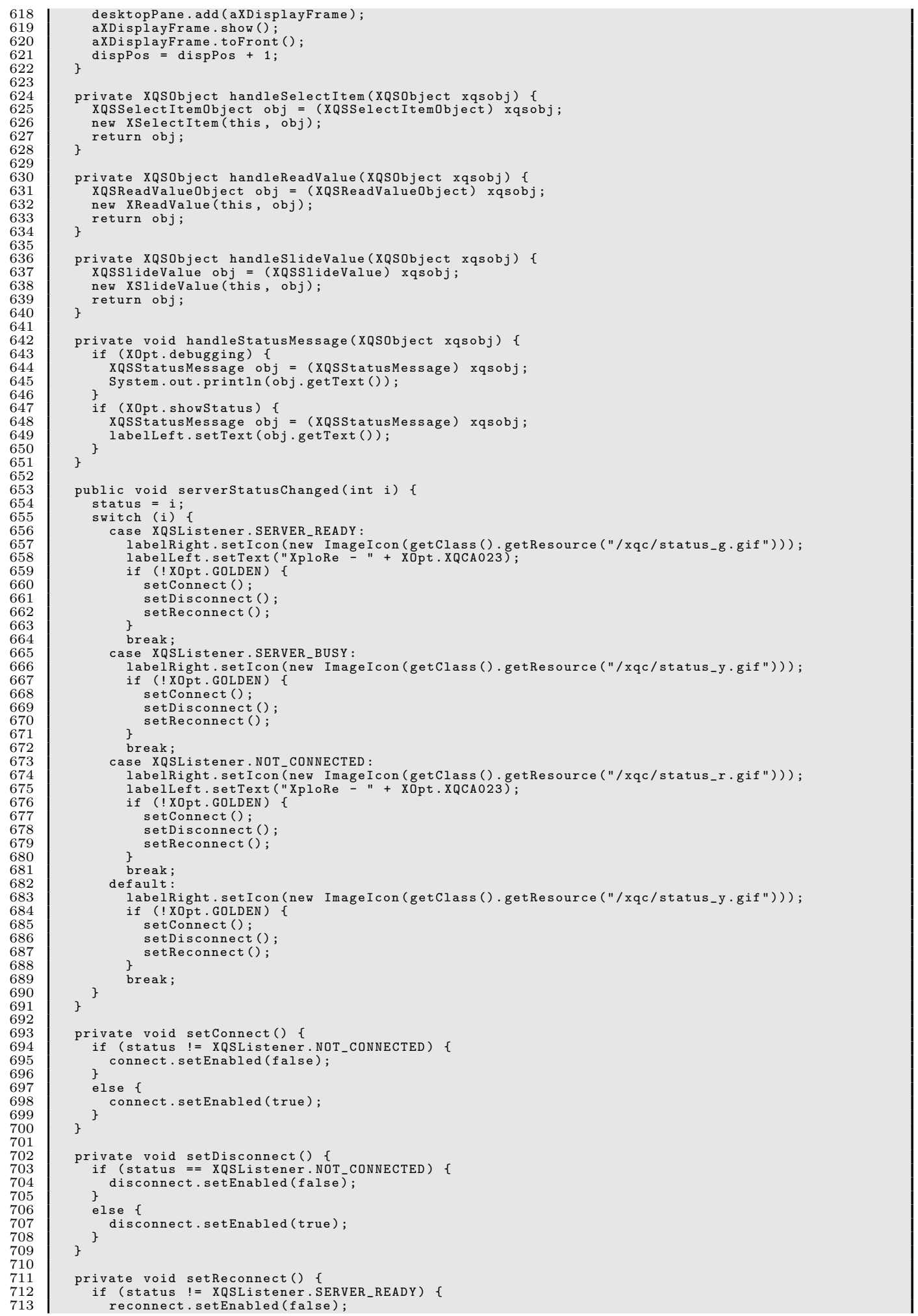




\section{B.1 XClient.java}

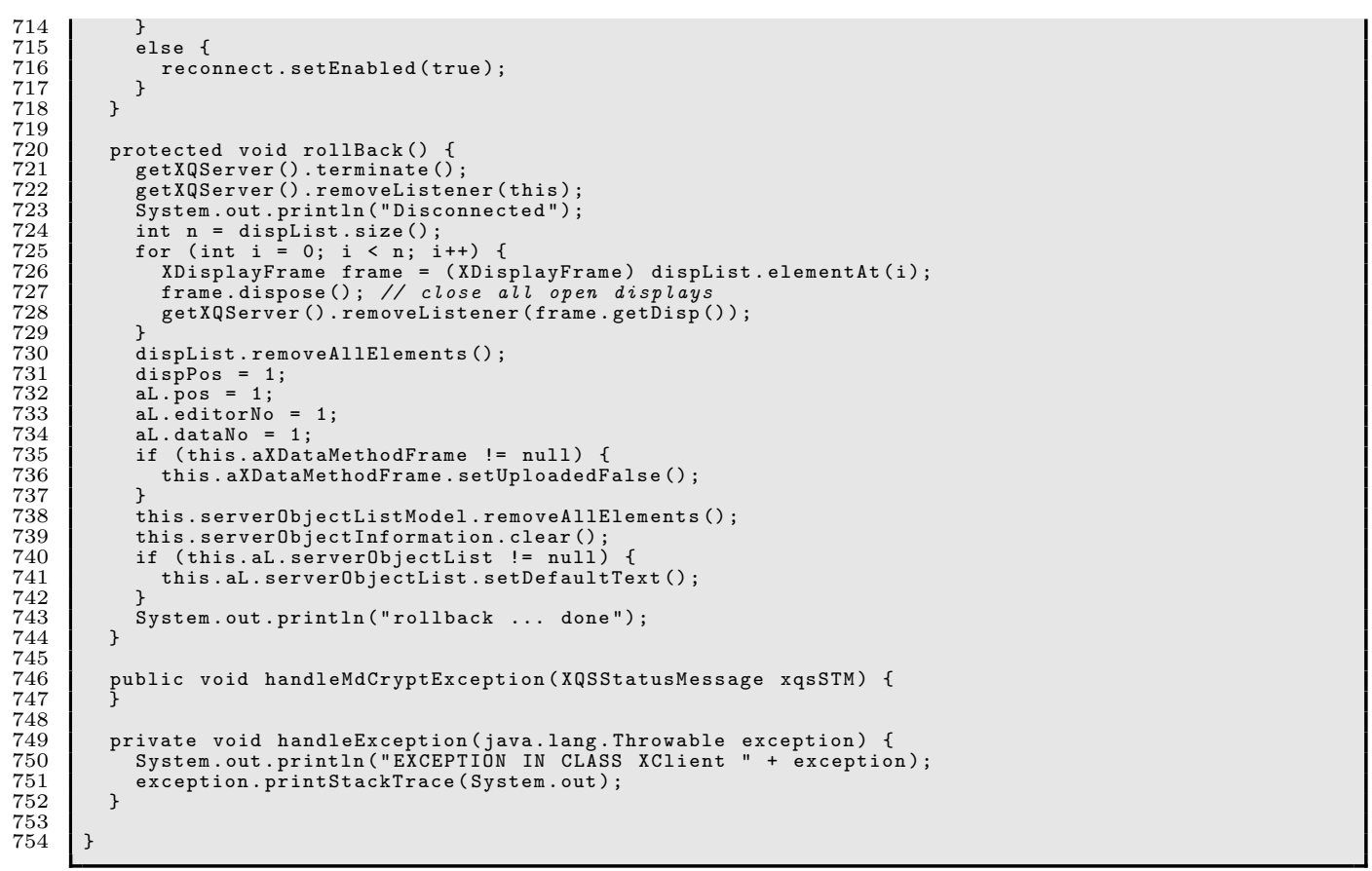




\section{B.2 XClientAction.java}

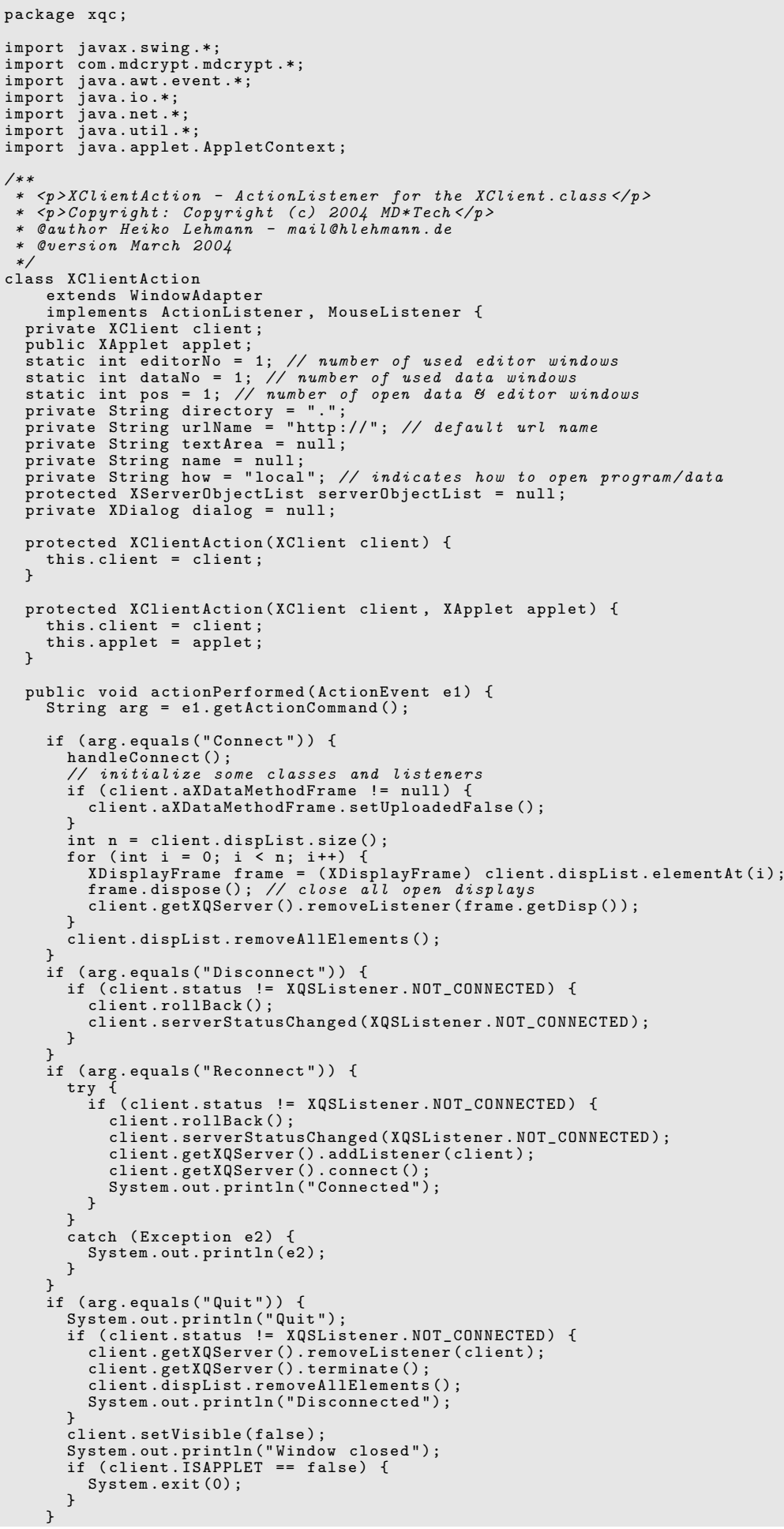




\section{B.2 XClientAction.java}

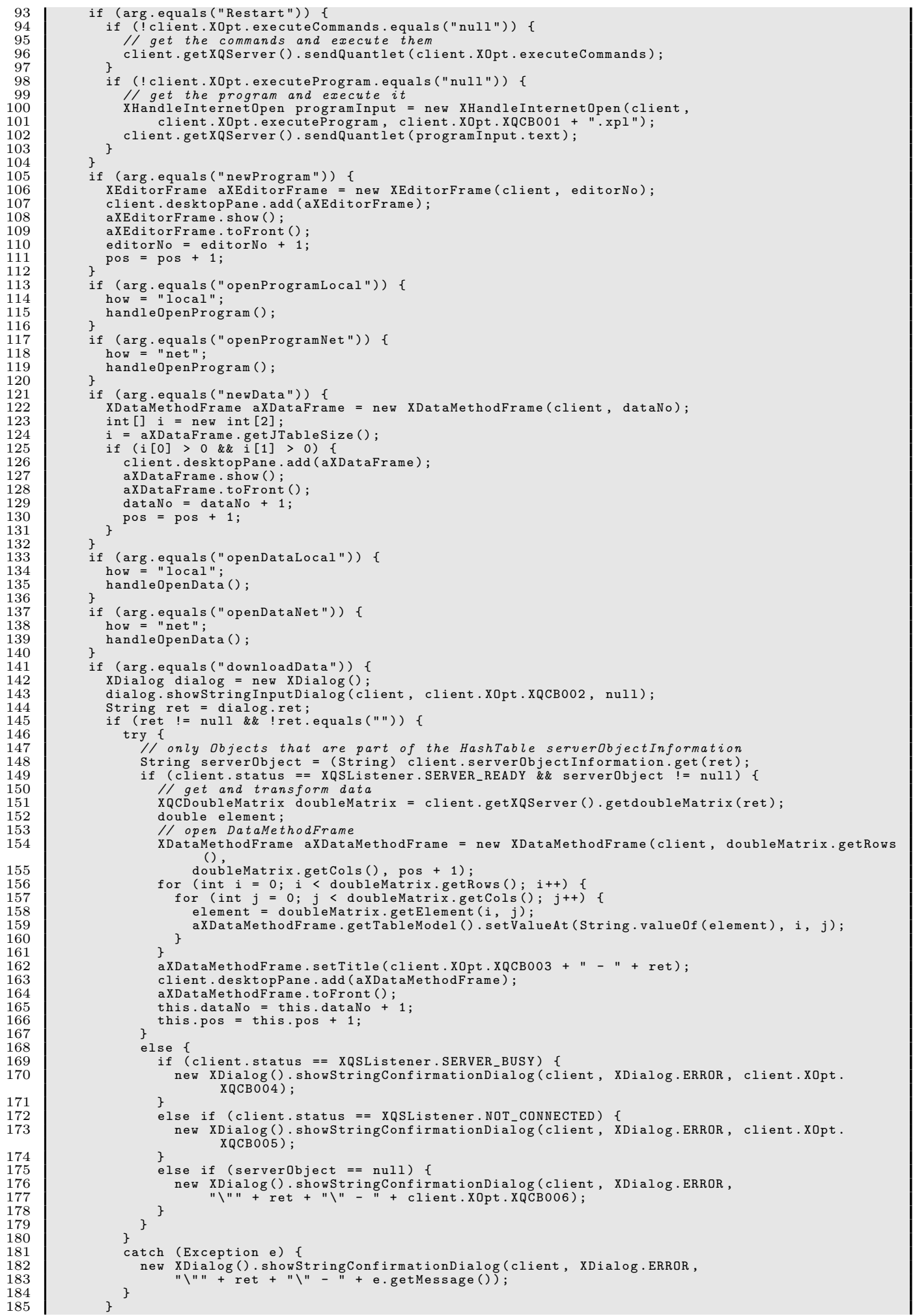




\section{XQC Source Code}

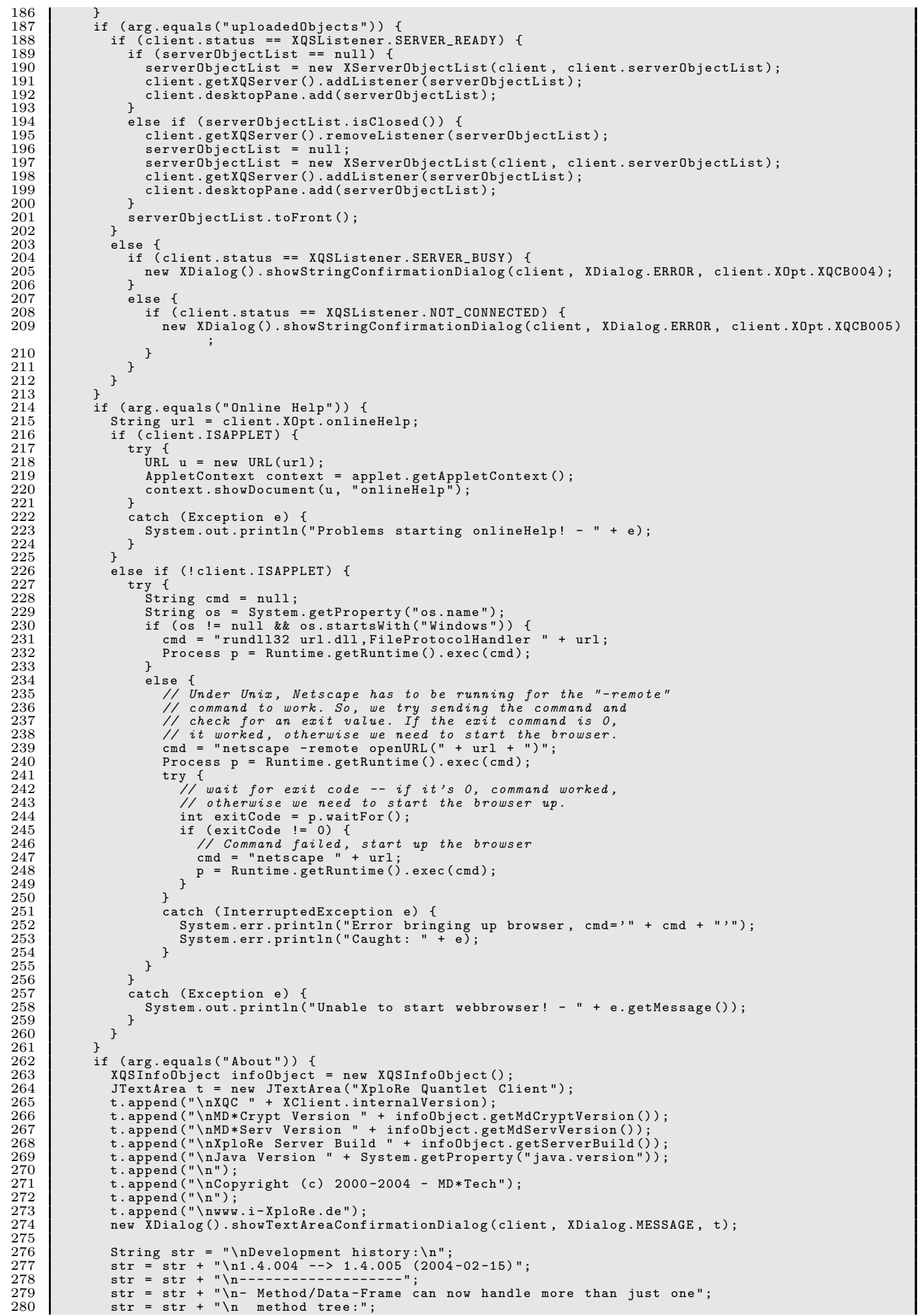




\section{B.2 XClientAction.java}

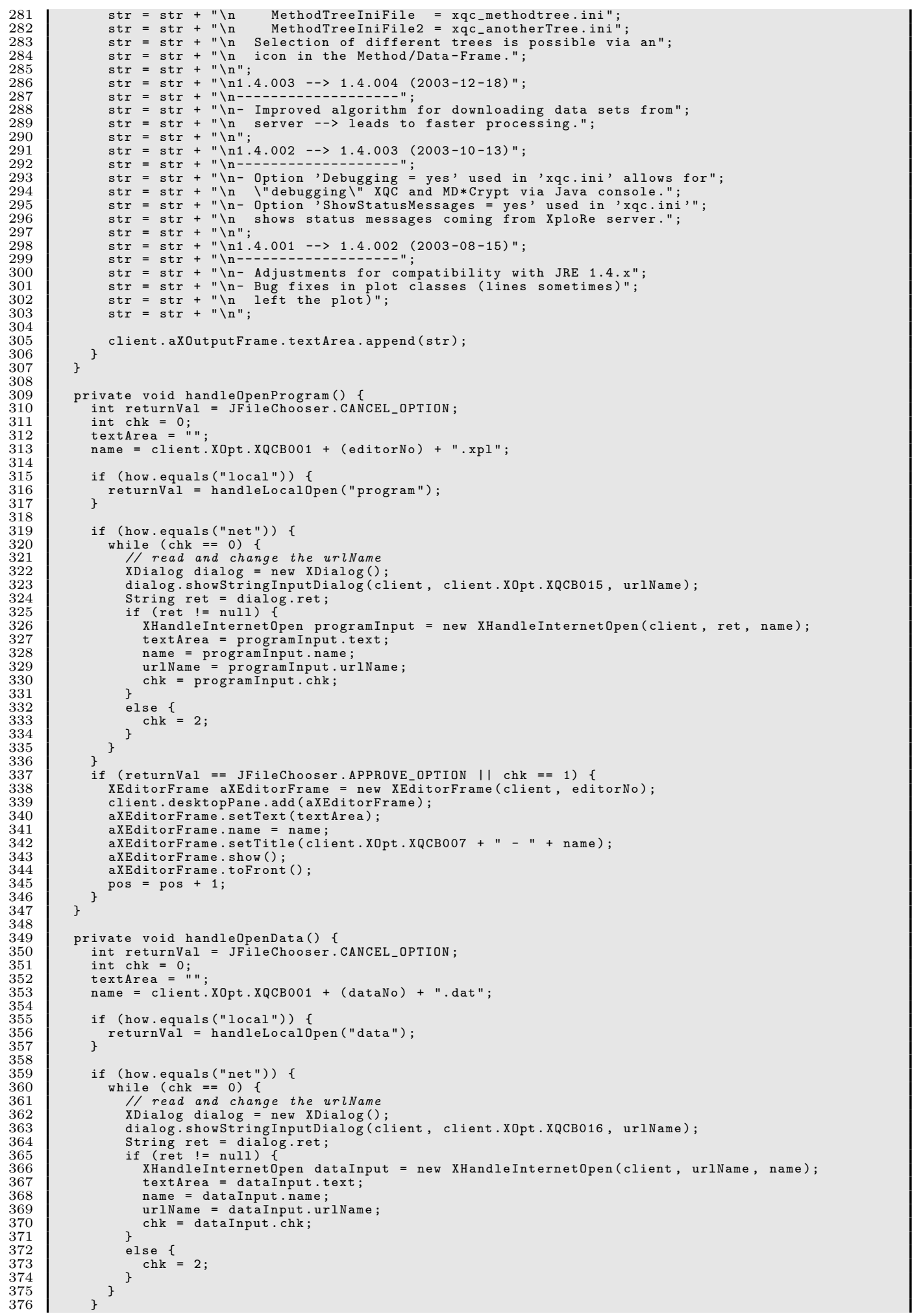




\section{XQC Source Code}

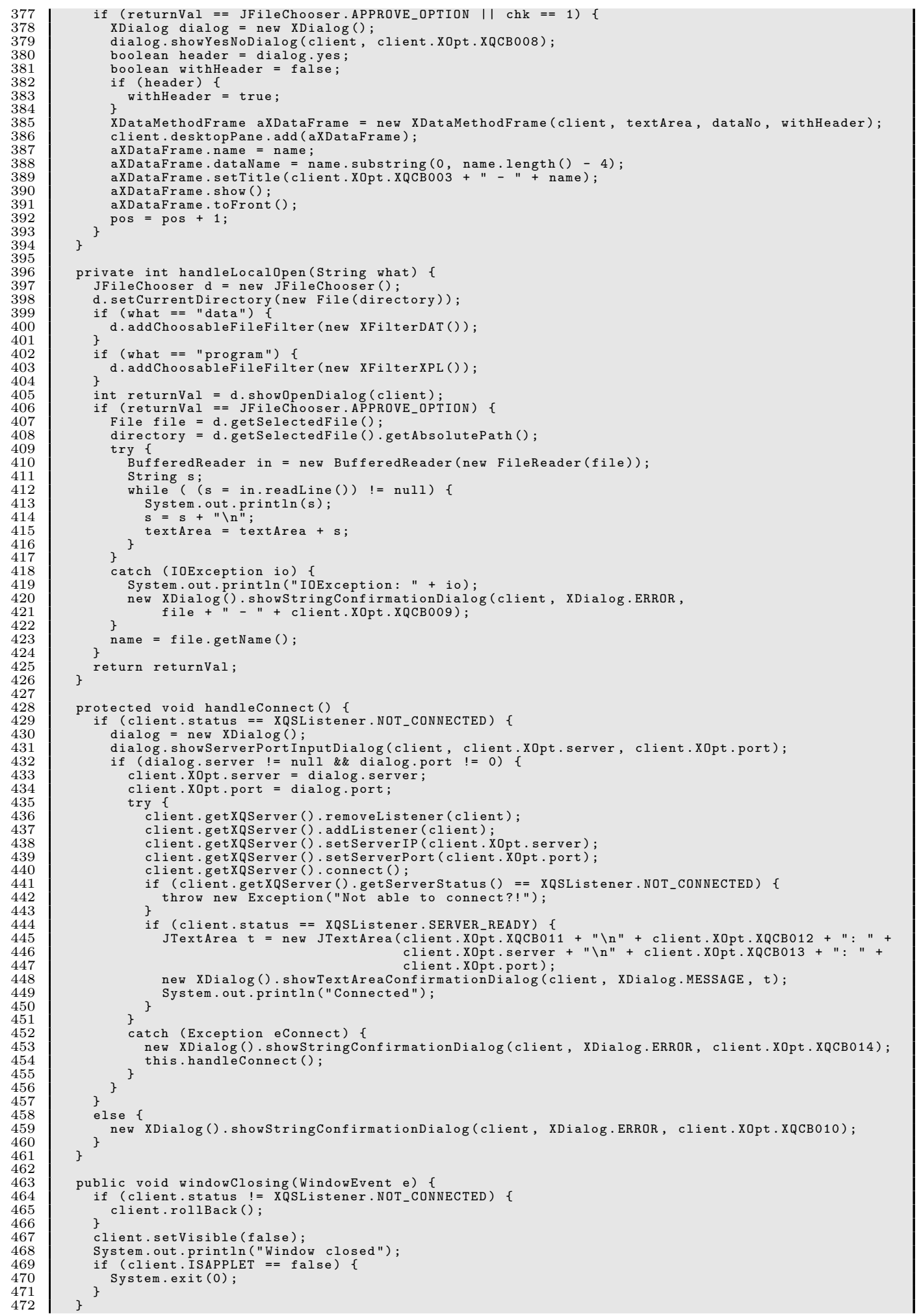




\section{B.2 XClientAction.java}

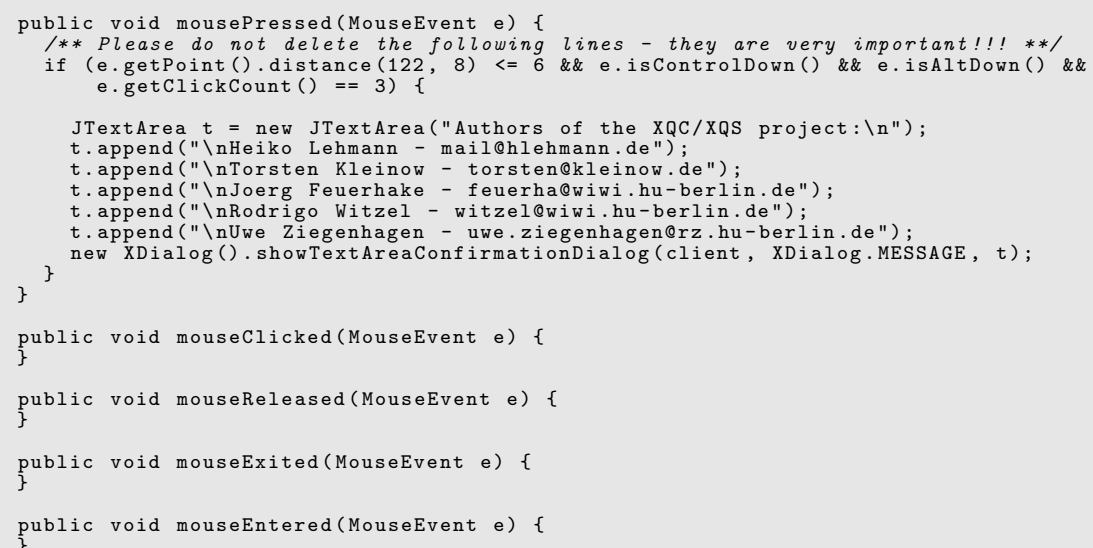




\section{B.3 XProperties.java}

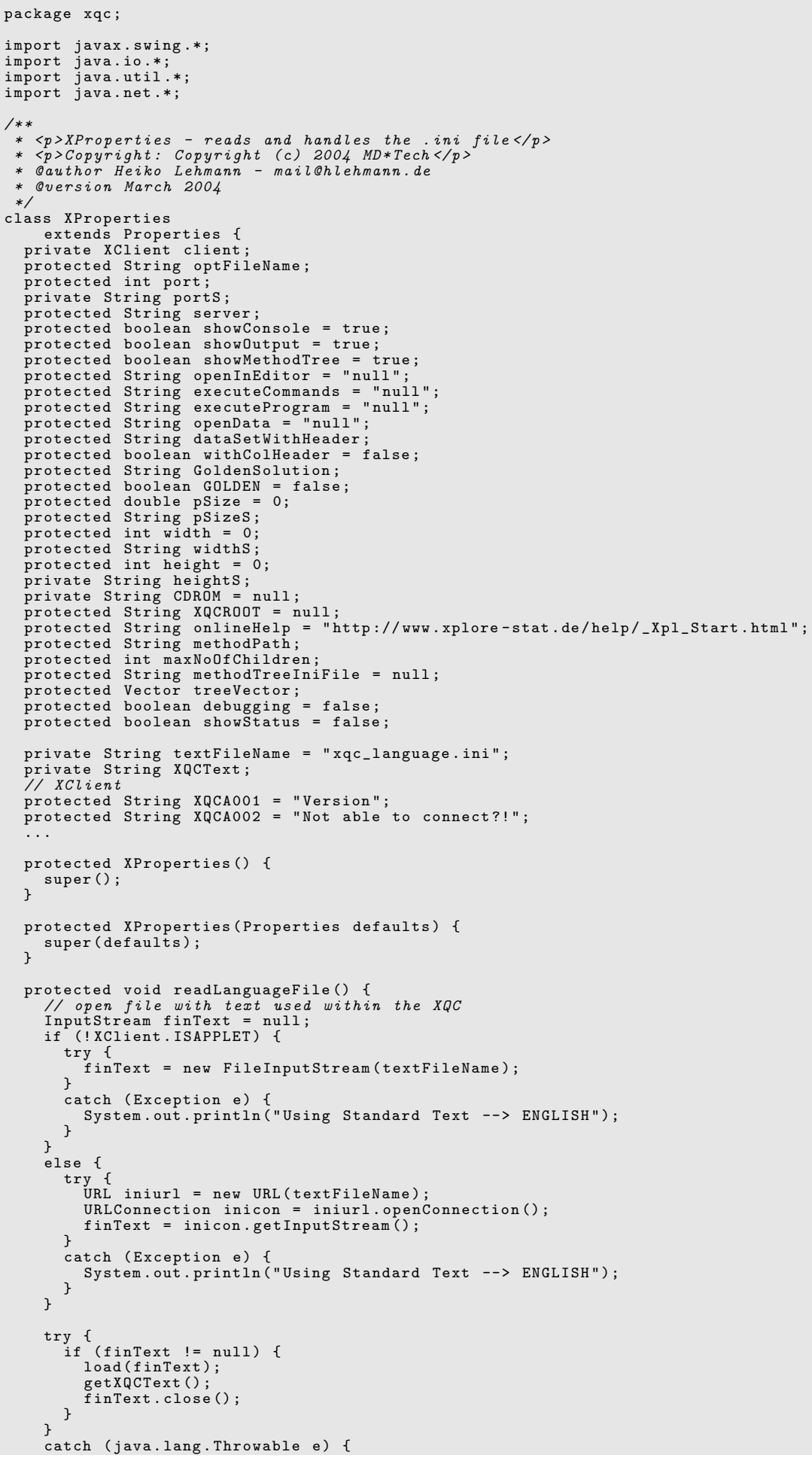




\section{B.3 XProperties.java}

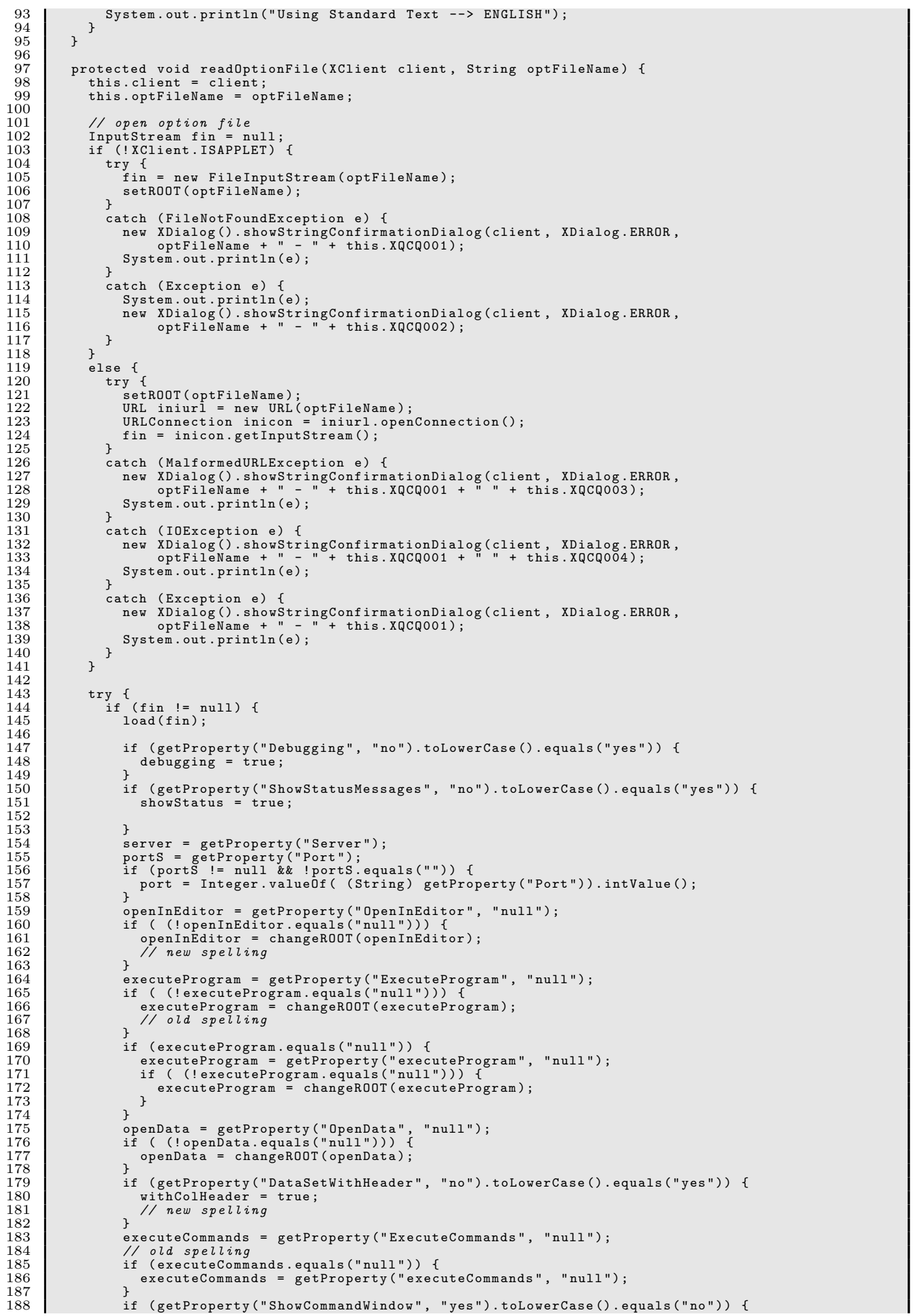




\section{XQC Source Code}

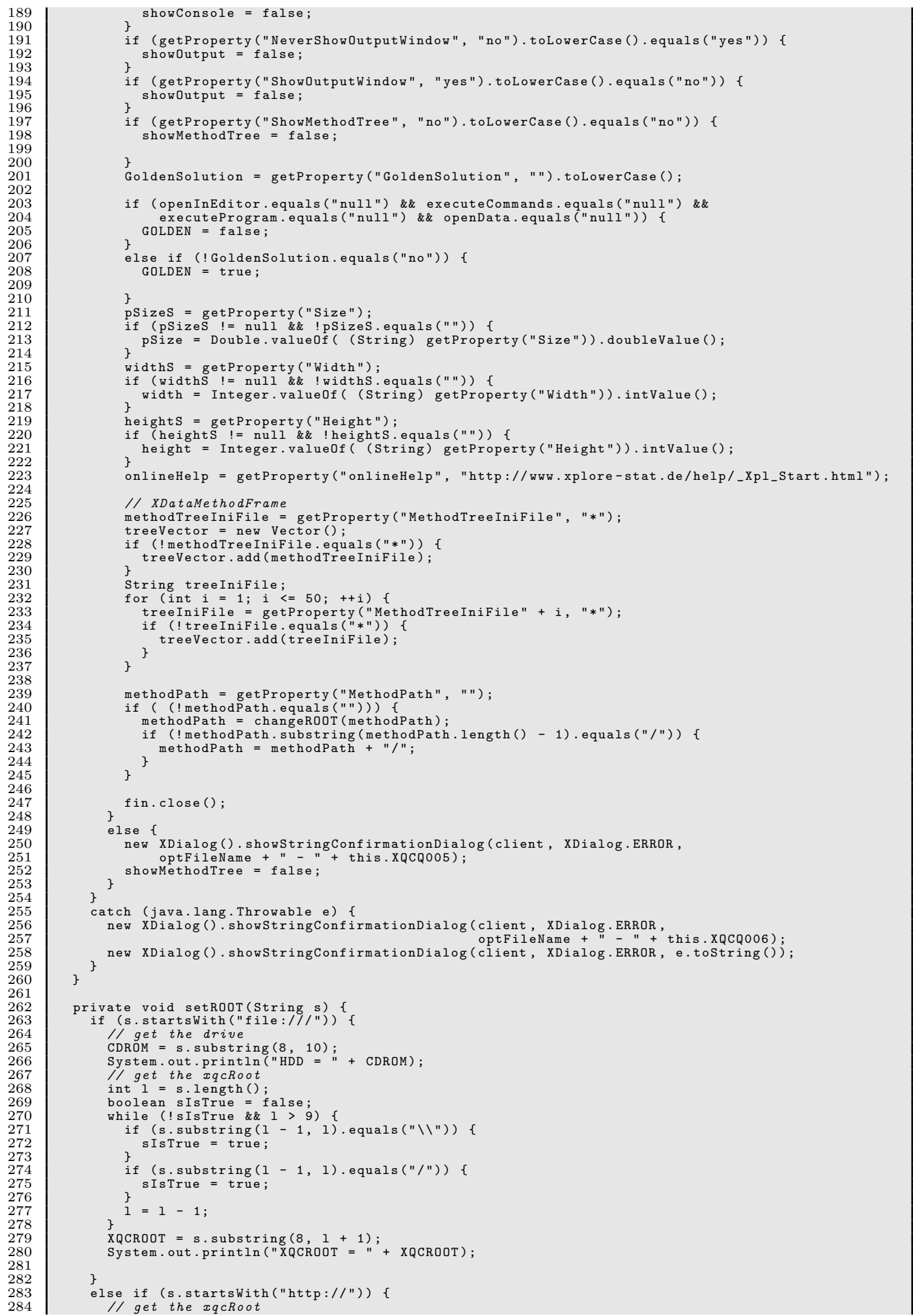




\section{B.3 XProperties.java}

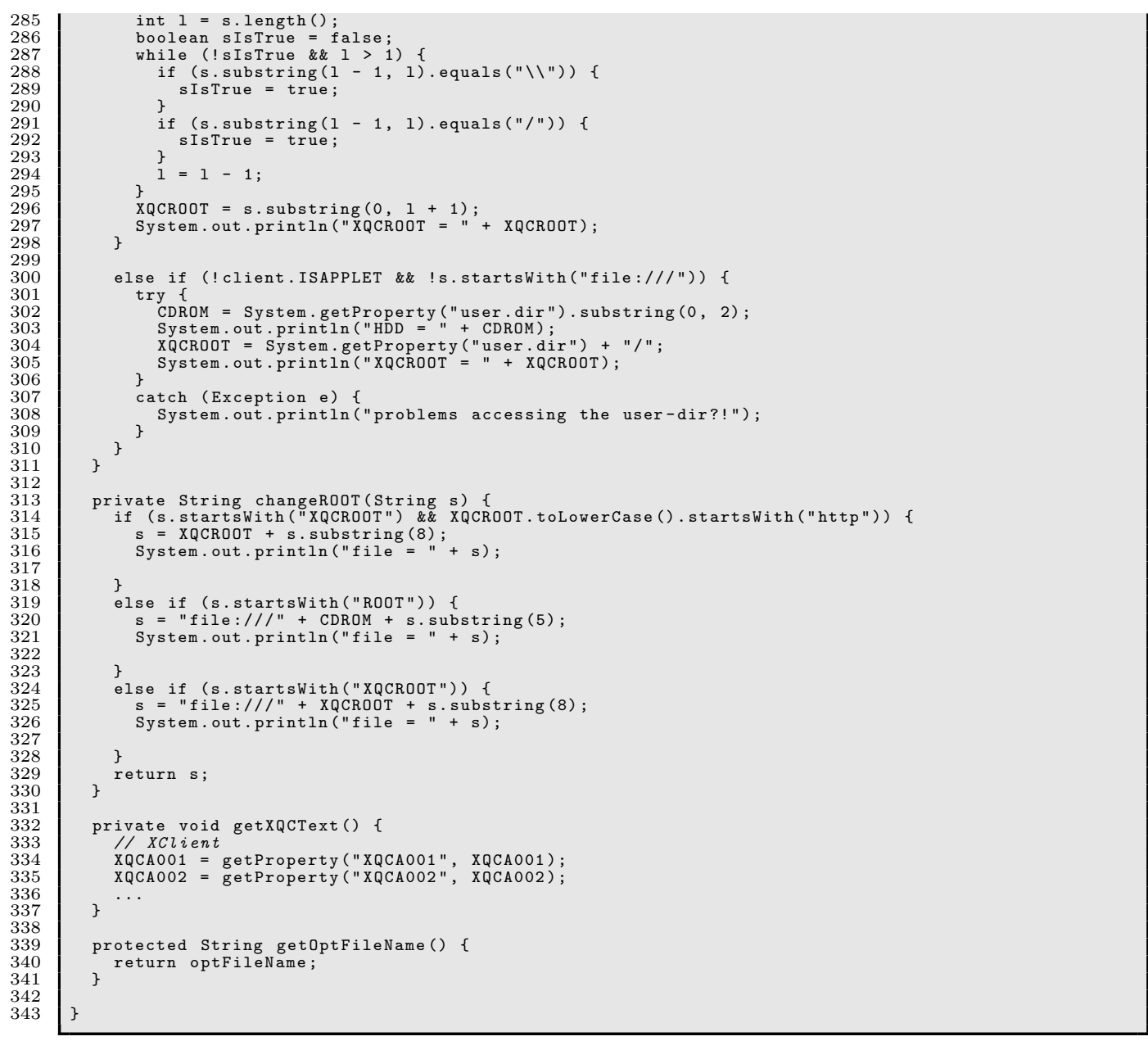




\section{B.4 XApplet.java}

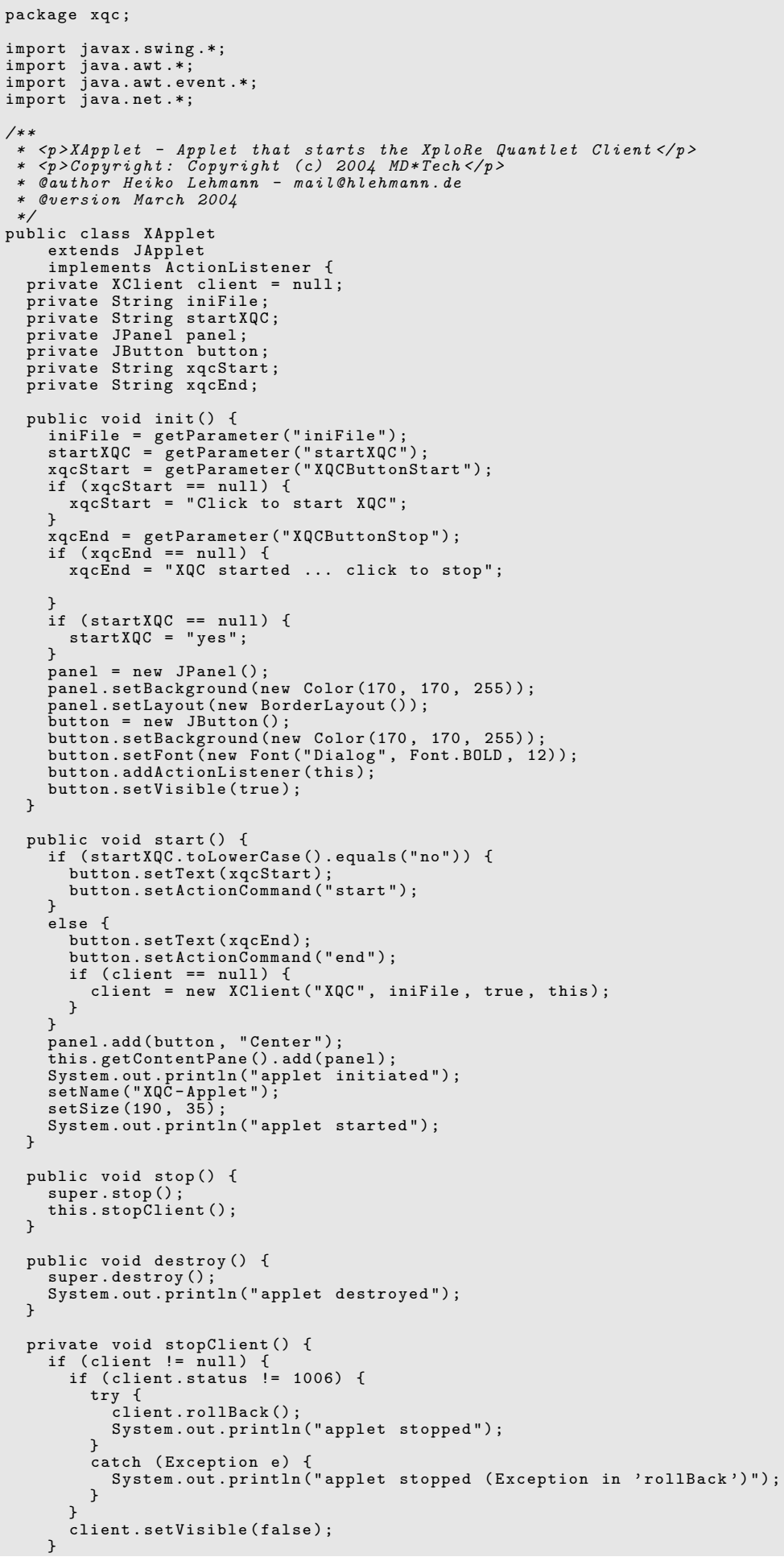


B.4 XApplet.java

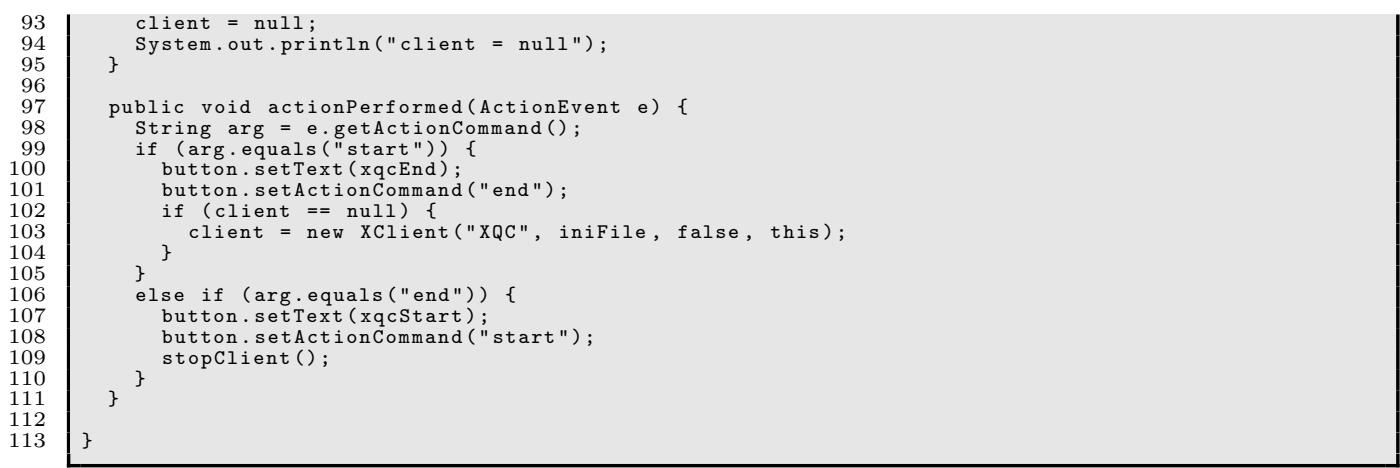




\section{B.5 XConsole.java}

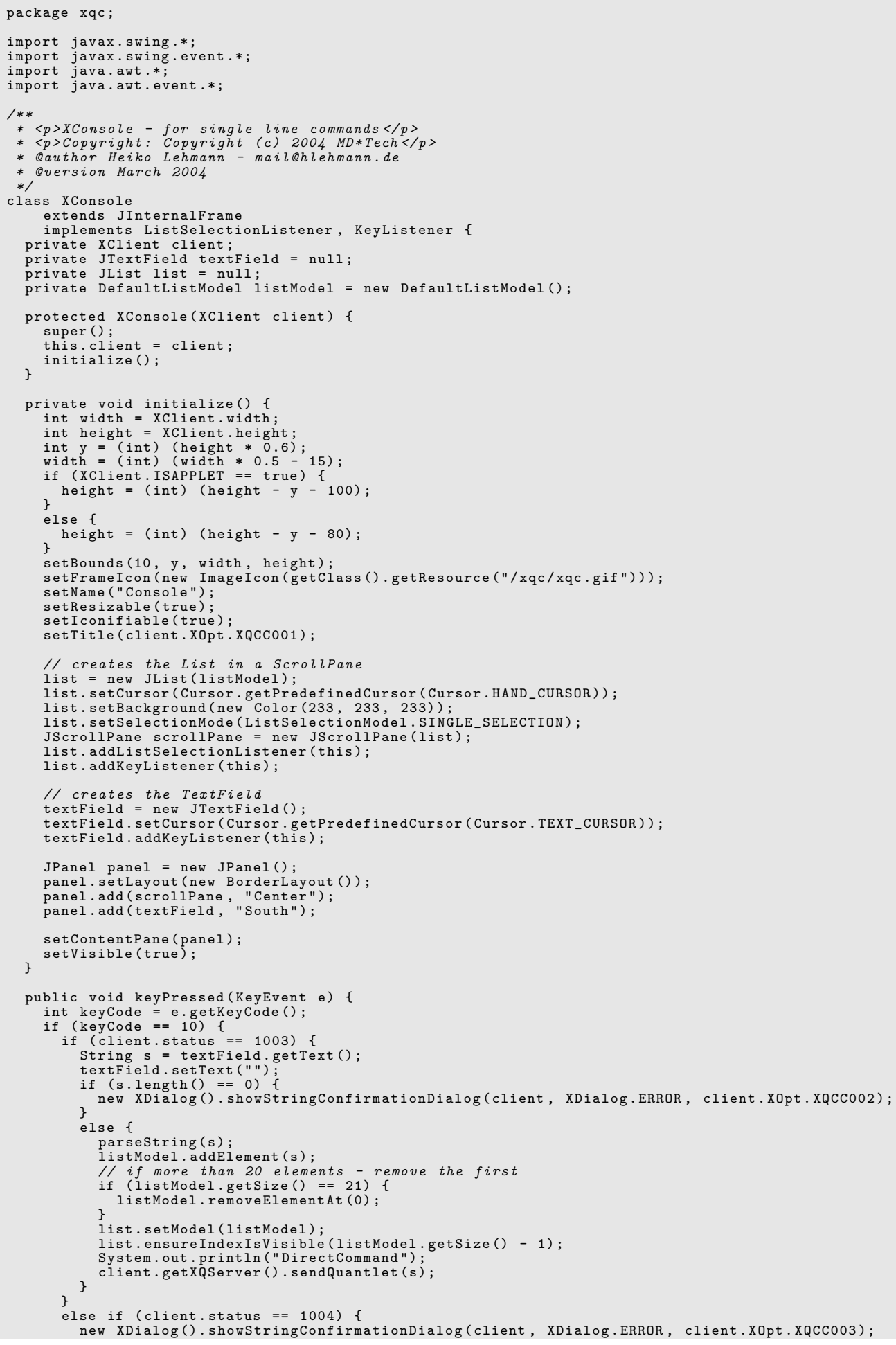




\section{B.5 XConsole.java}

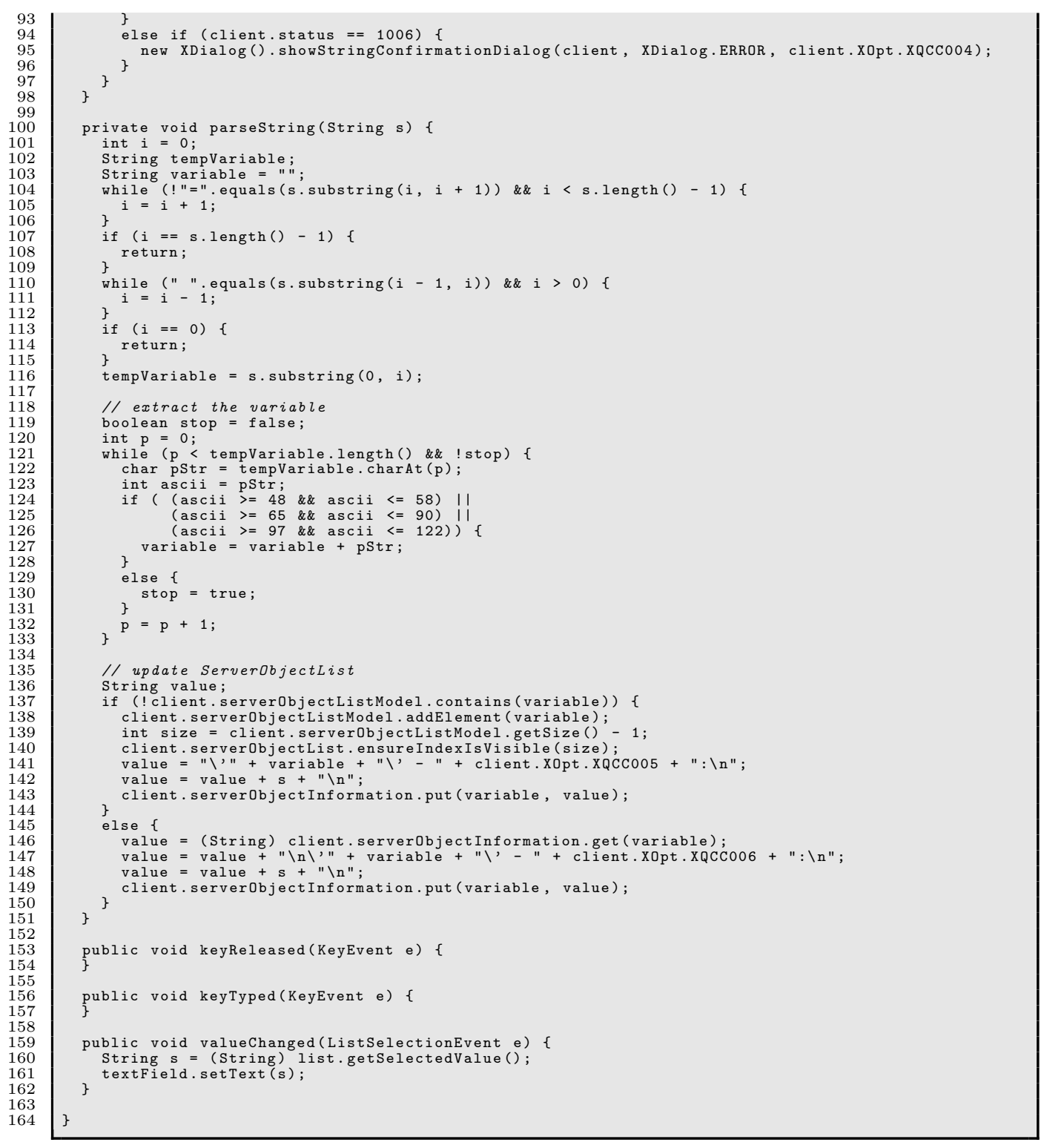




\section{B.6 XEditorFrame.java}

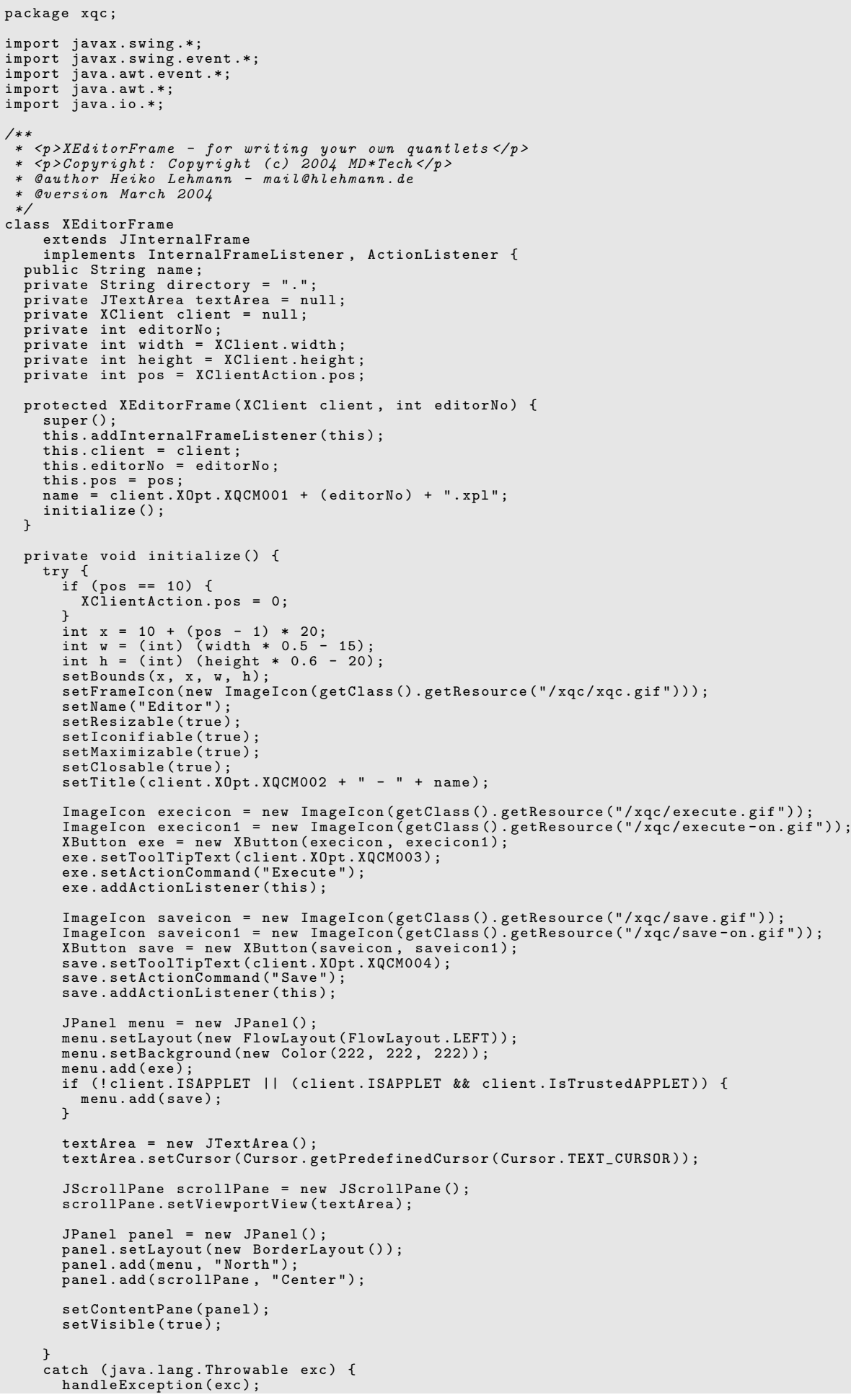




\section{B.6 XEditorFrame.java}

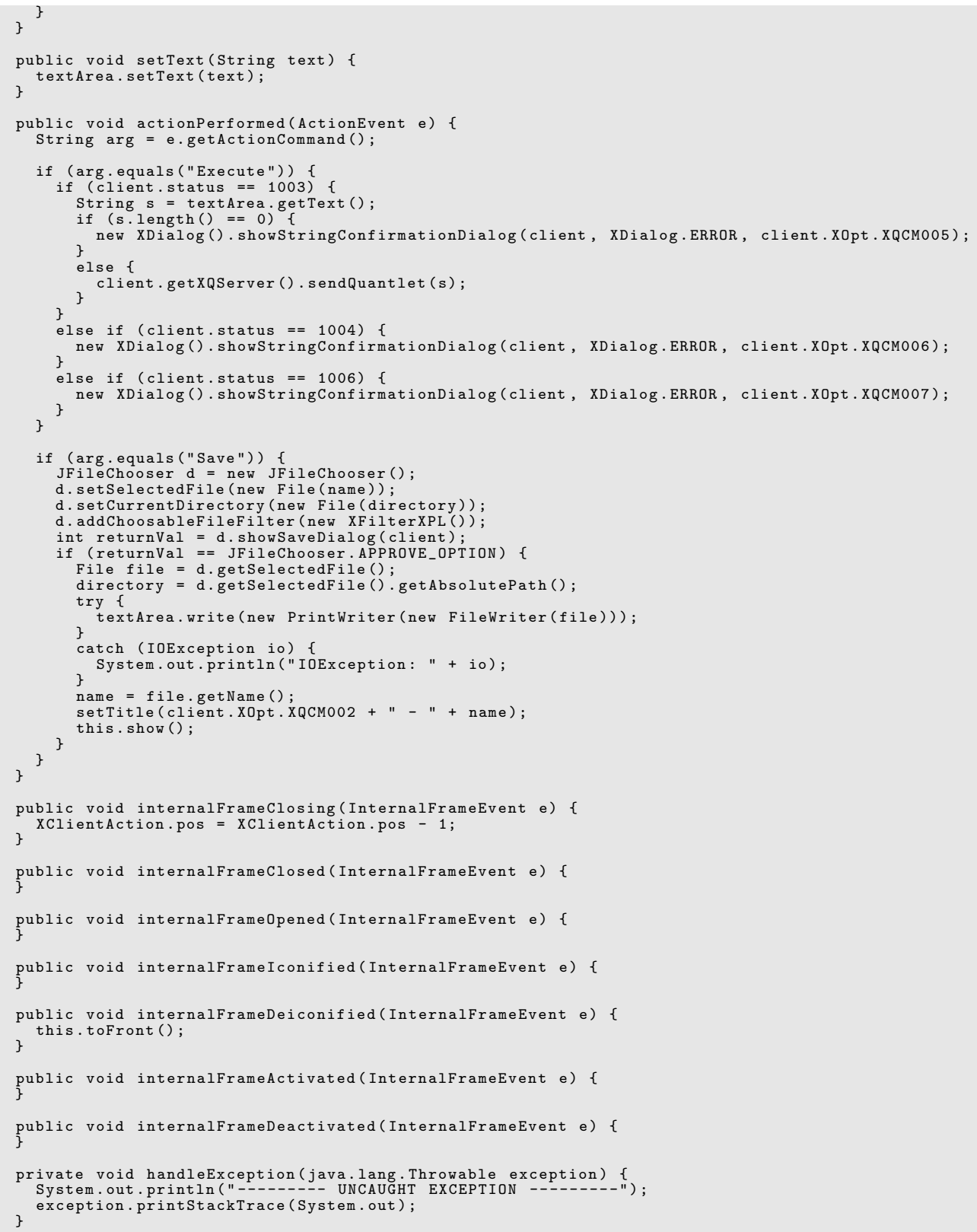




\section{B.7 XOutputFrame.java}

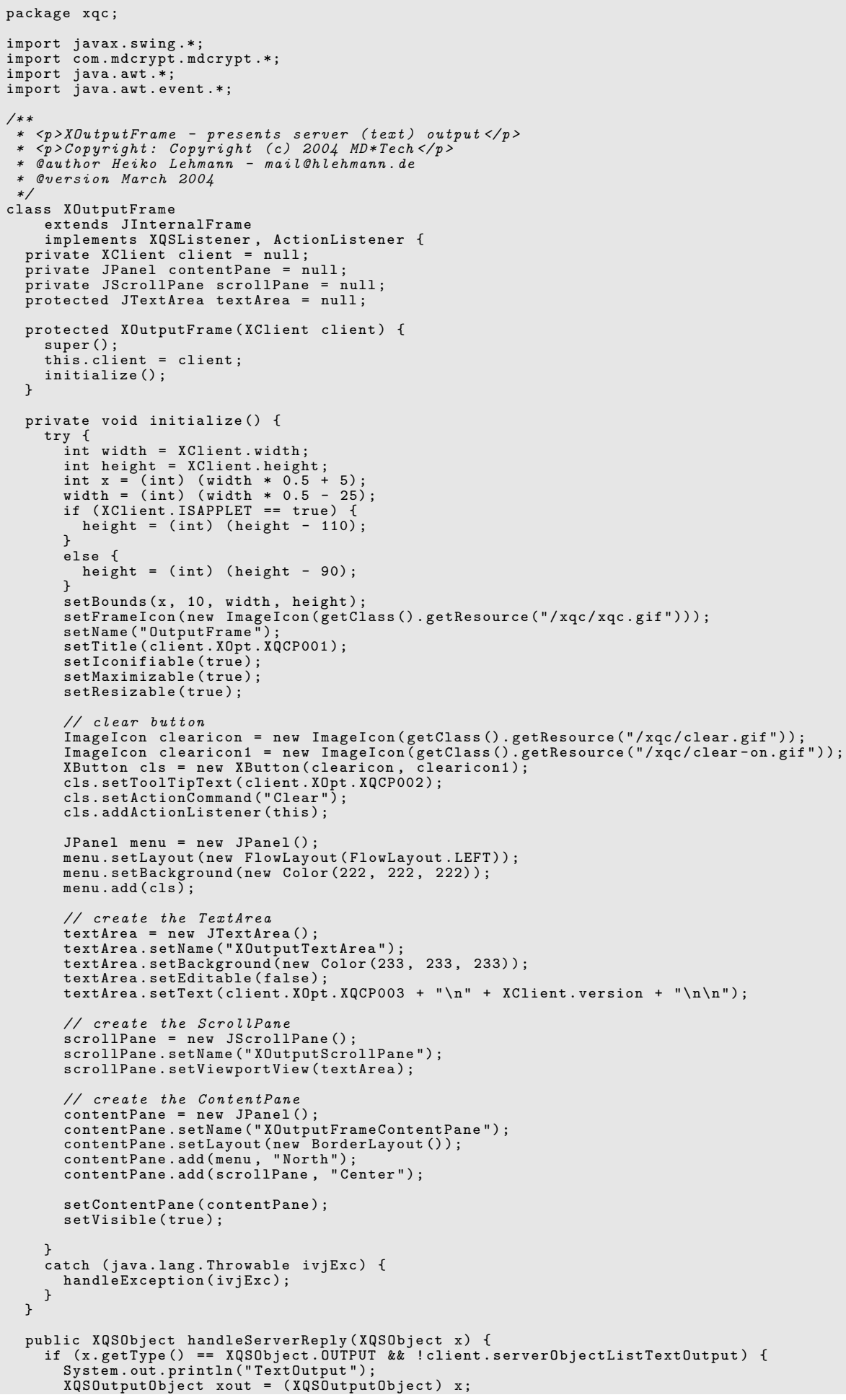




\section{B.7 XOutputFrame.java}

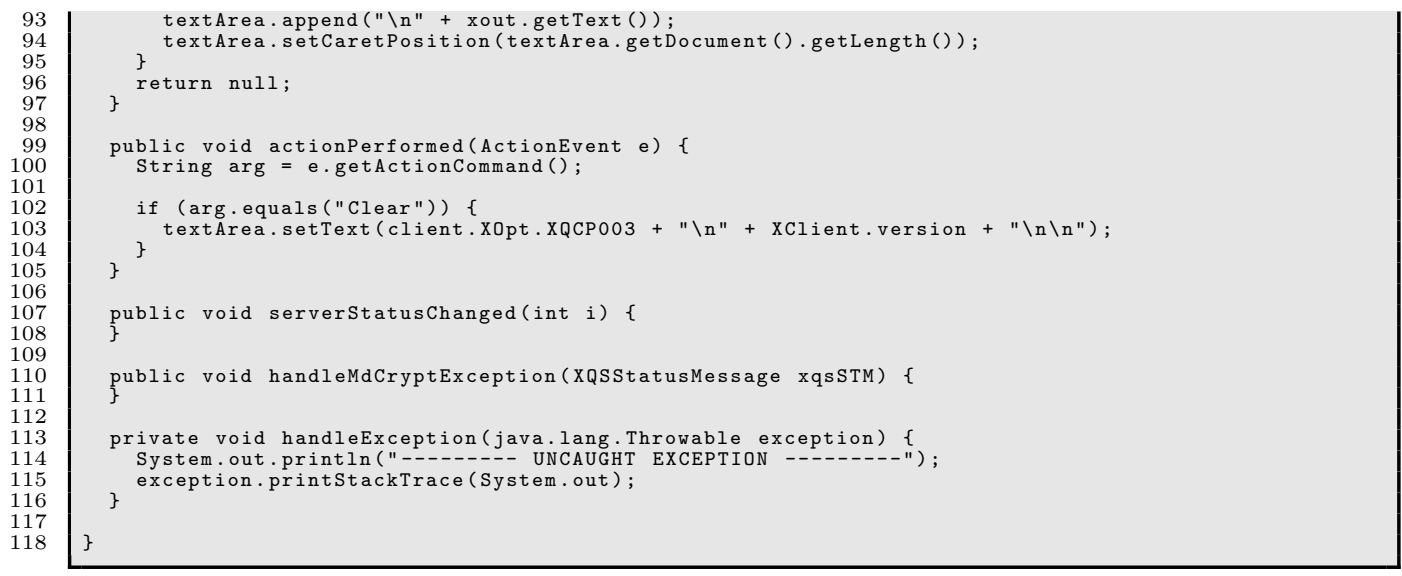




\section{B.8 XDataMethodFrame.java}

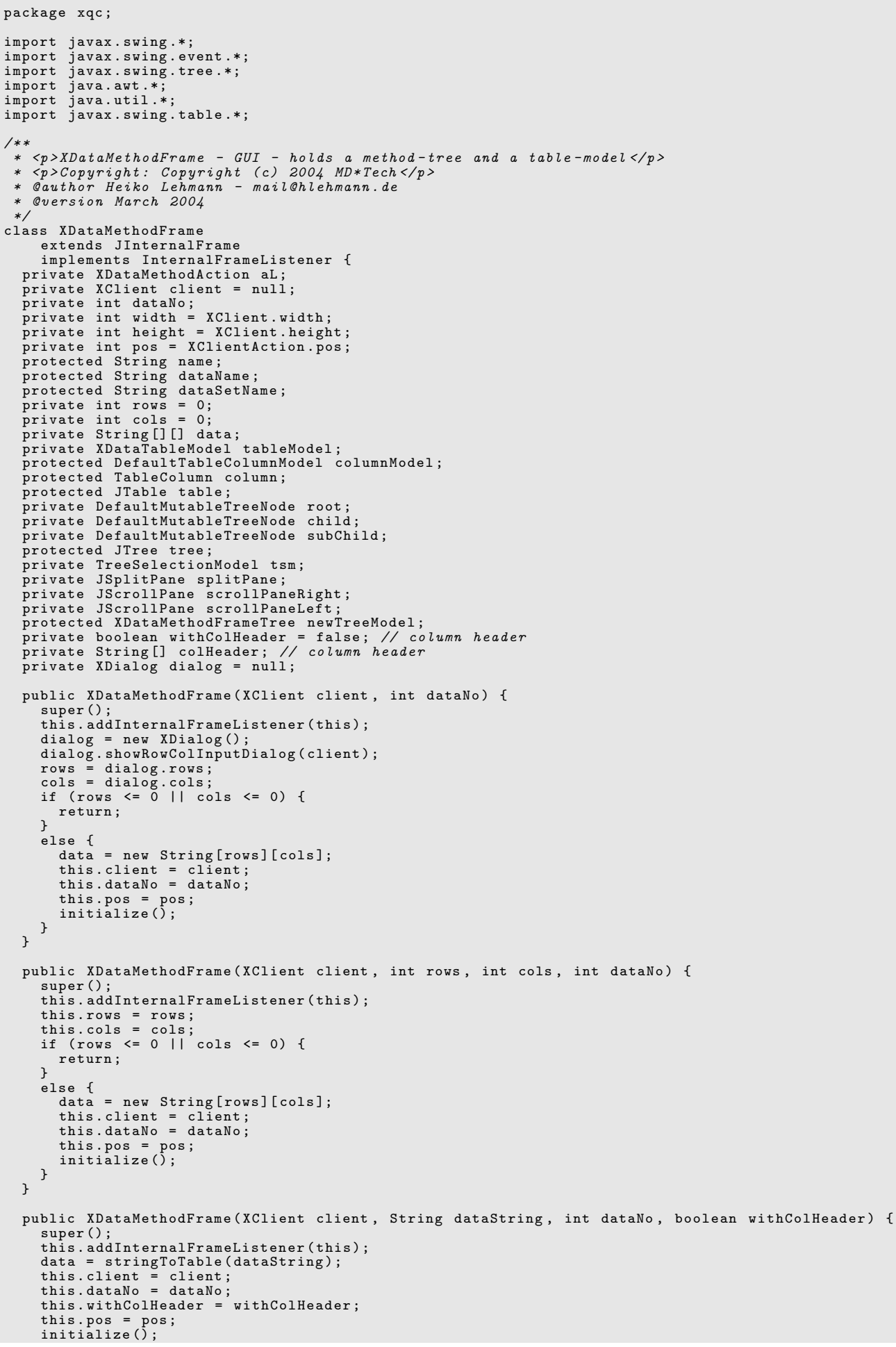




\section{B.8 XDataMethodFrame.java}

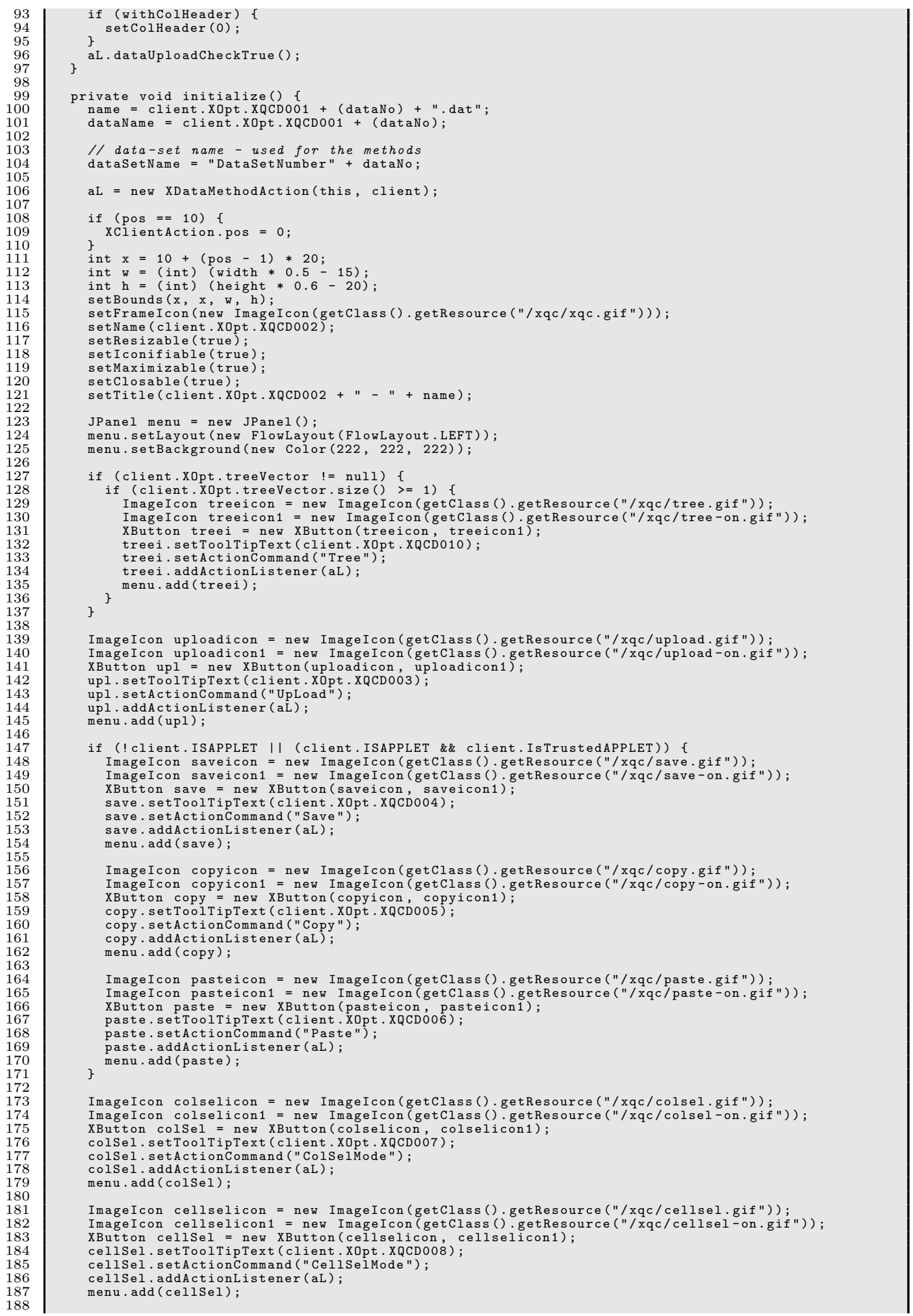




\section{XQC Source Code}

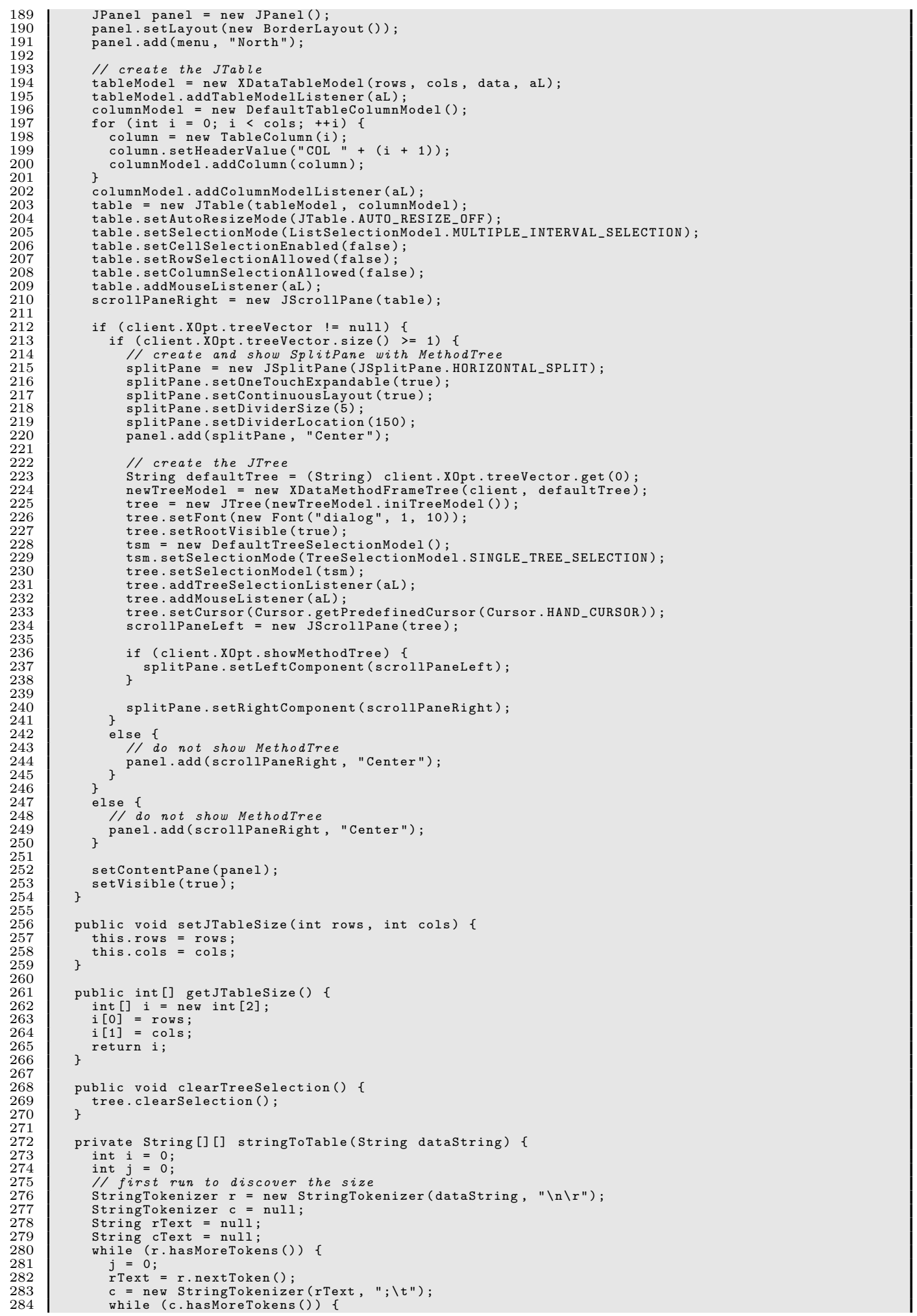




\section{B.8 XDataMethodFrame.java}

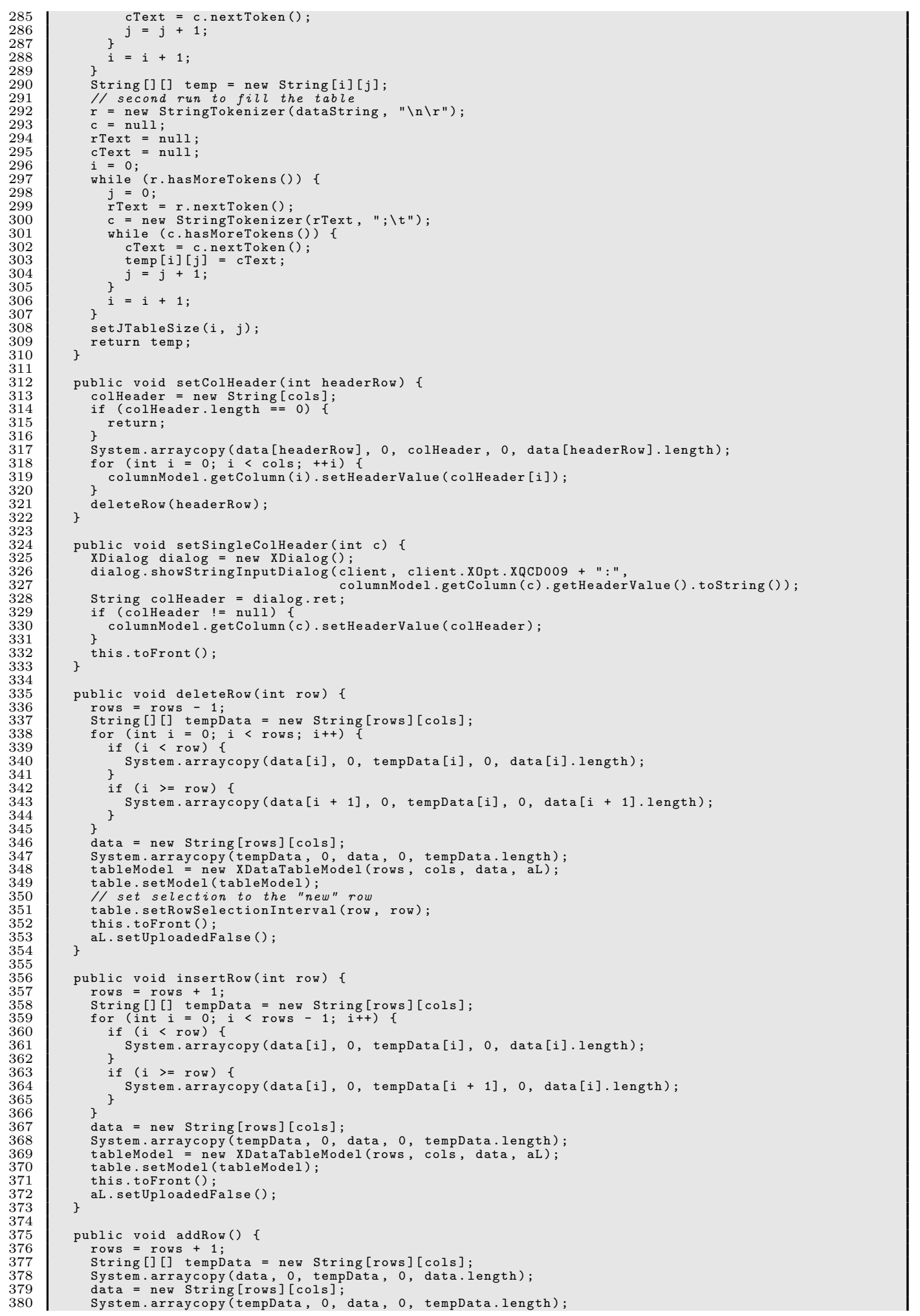




\section{XQC Source Code}

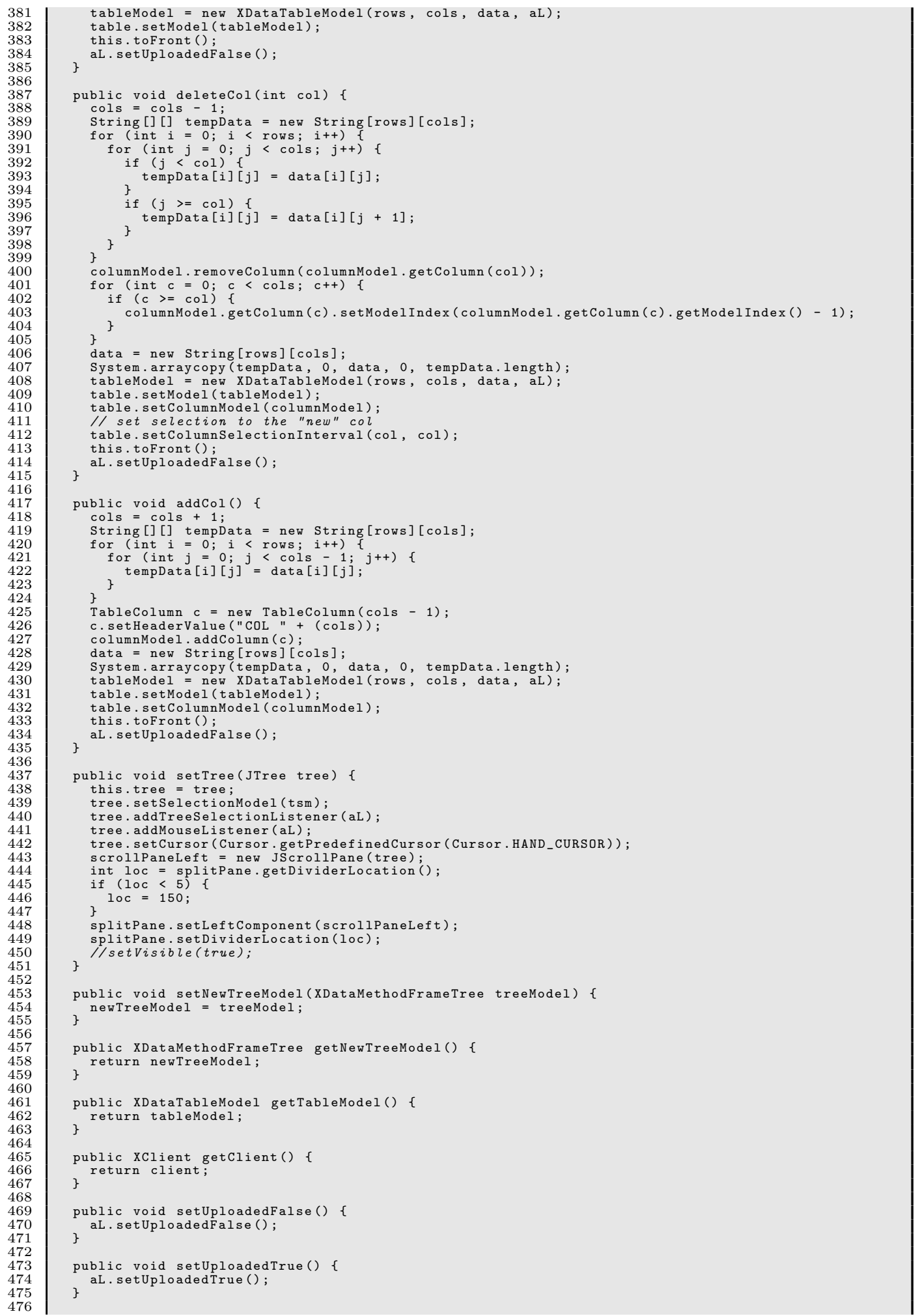




\section{B.8 XDataMethodFrame.java}

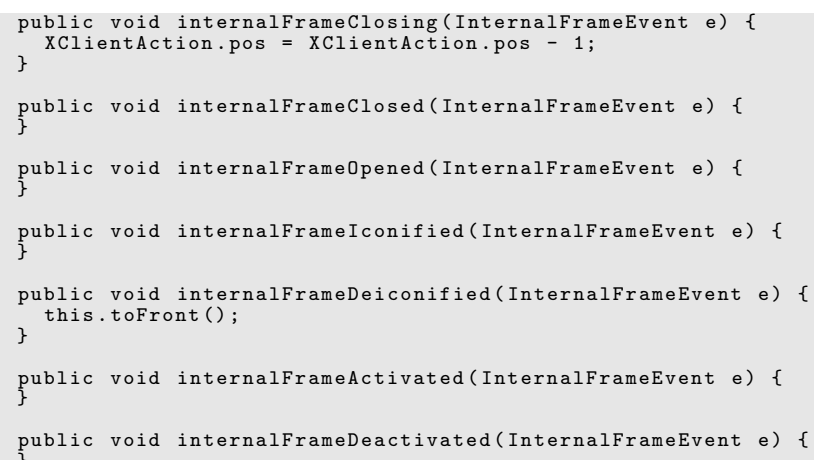




\section{B.9 XDataMethodAction.java}

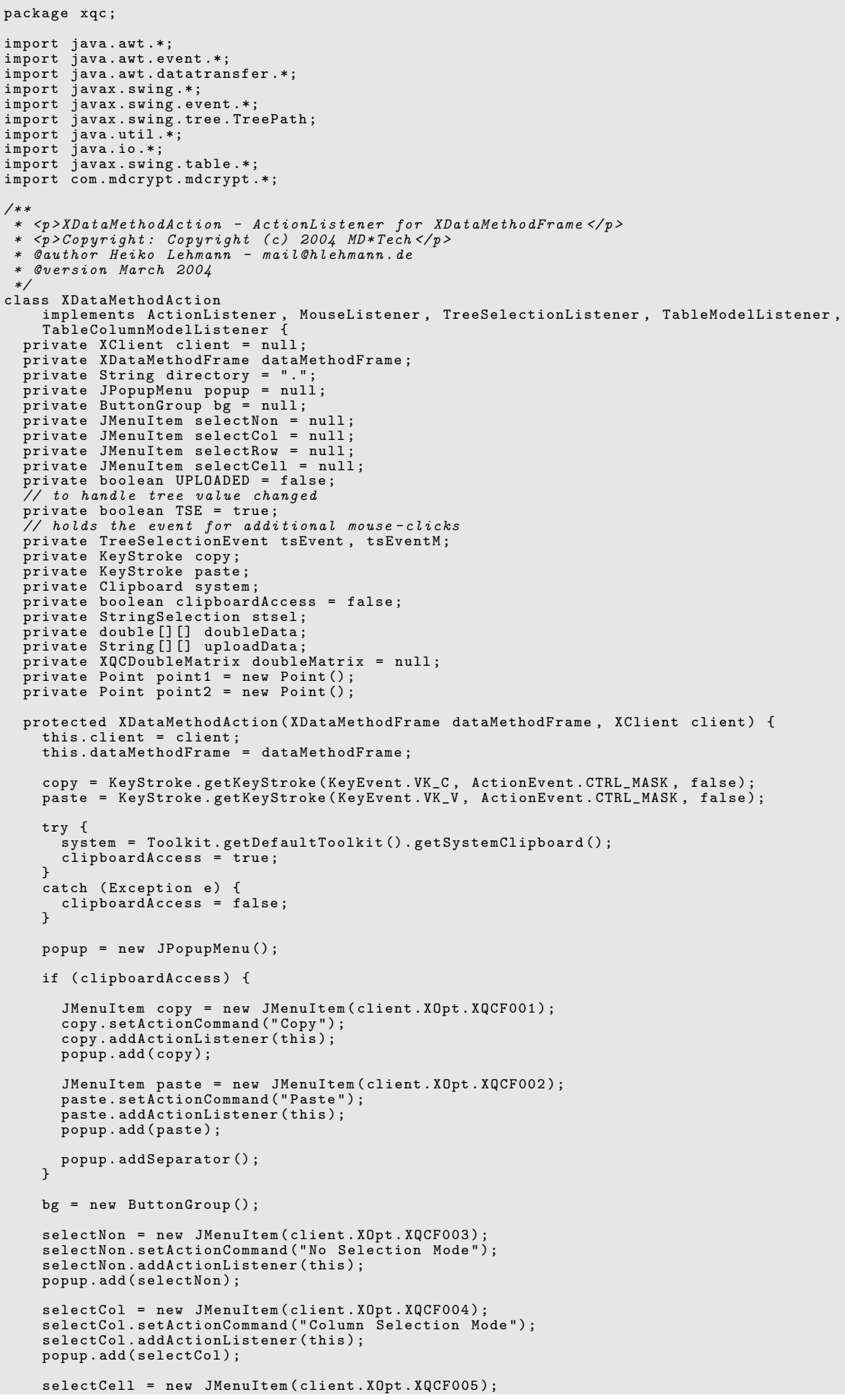

selectCell = new JMenuItem(client.XOpt.XQCF005); 


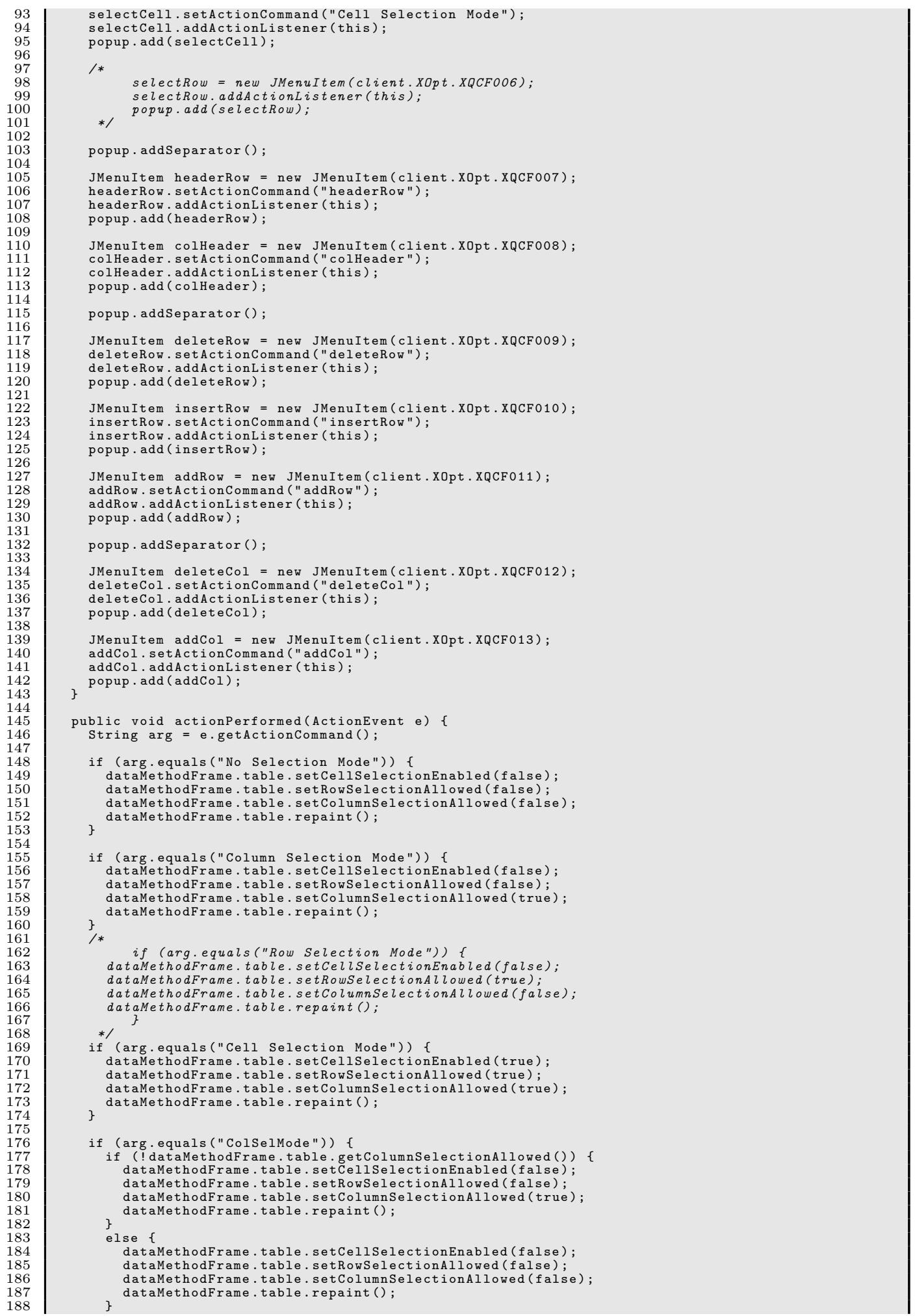




\section{XQC Source Code}

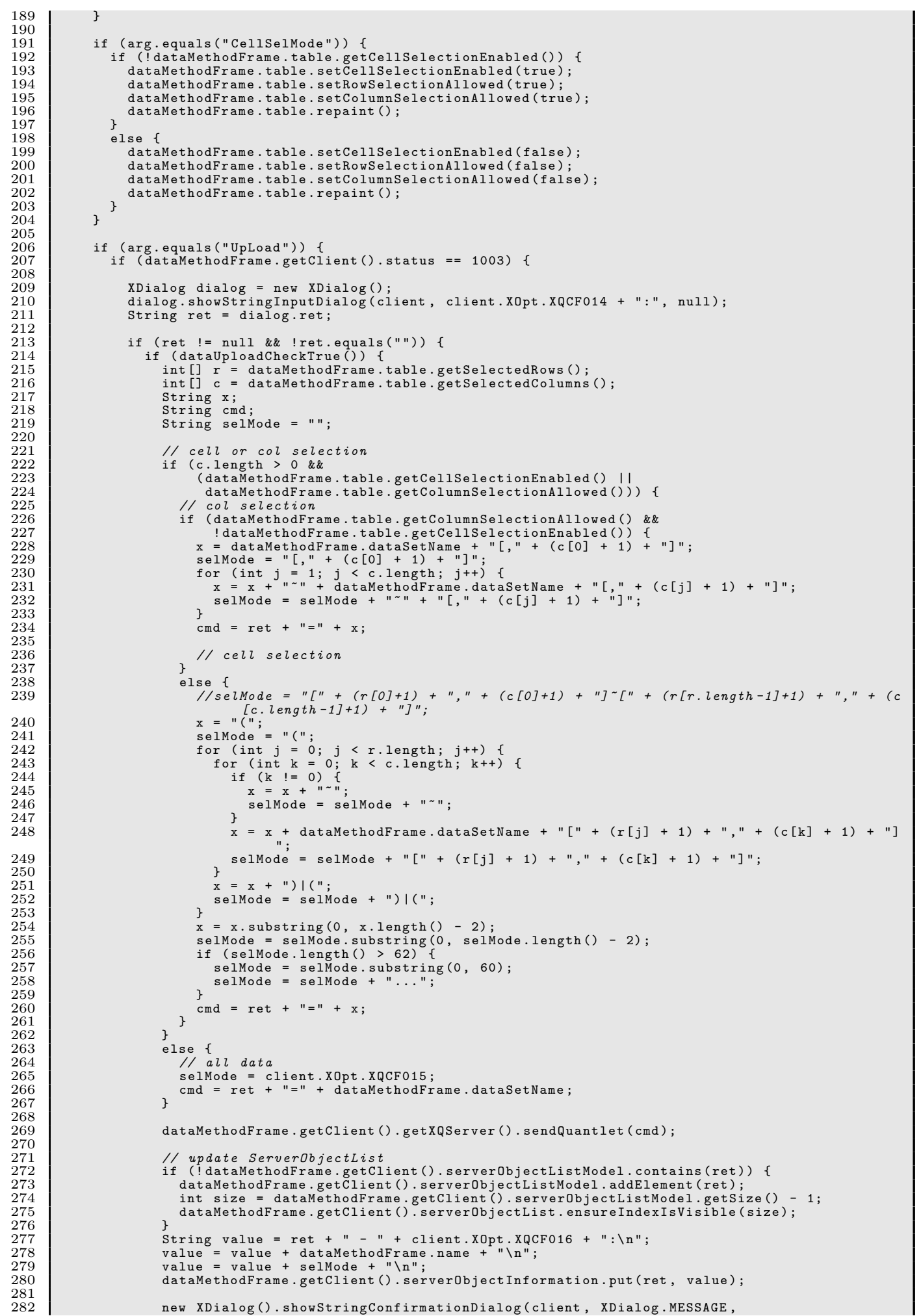




\section{B.9 XDataMethodAction.java}

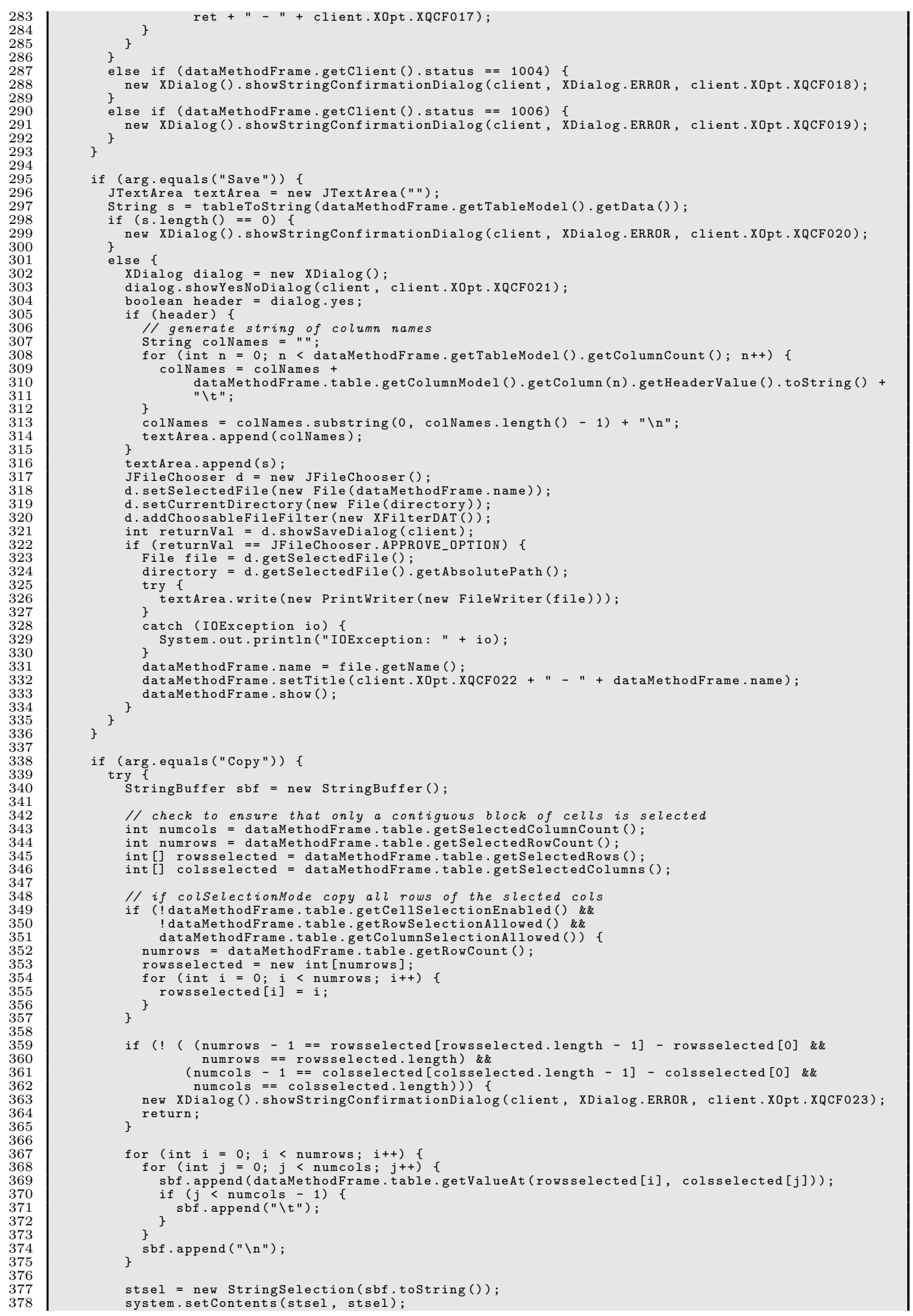




\section{XQC Source Code}

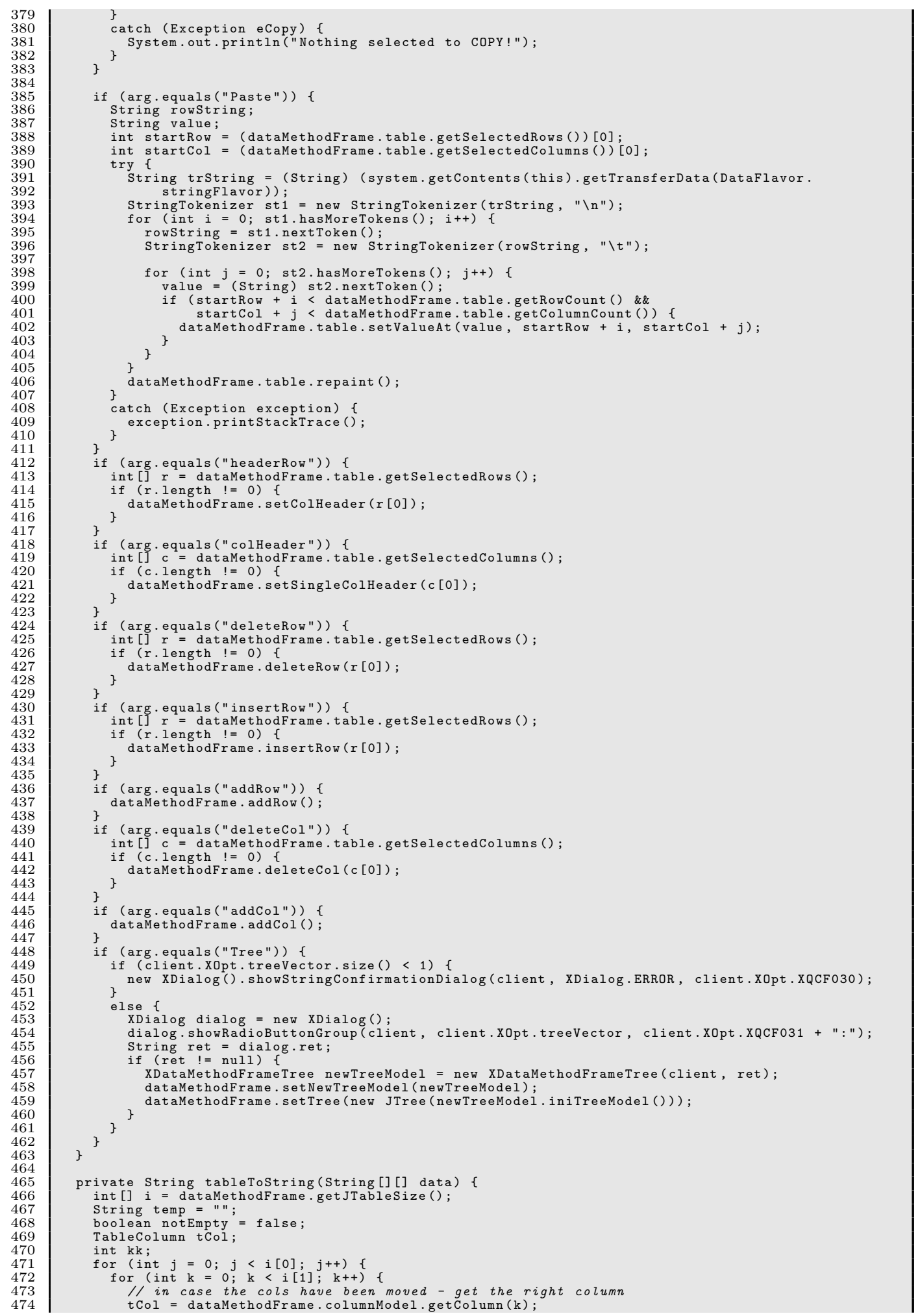




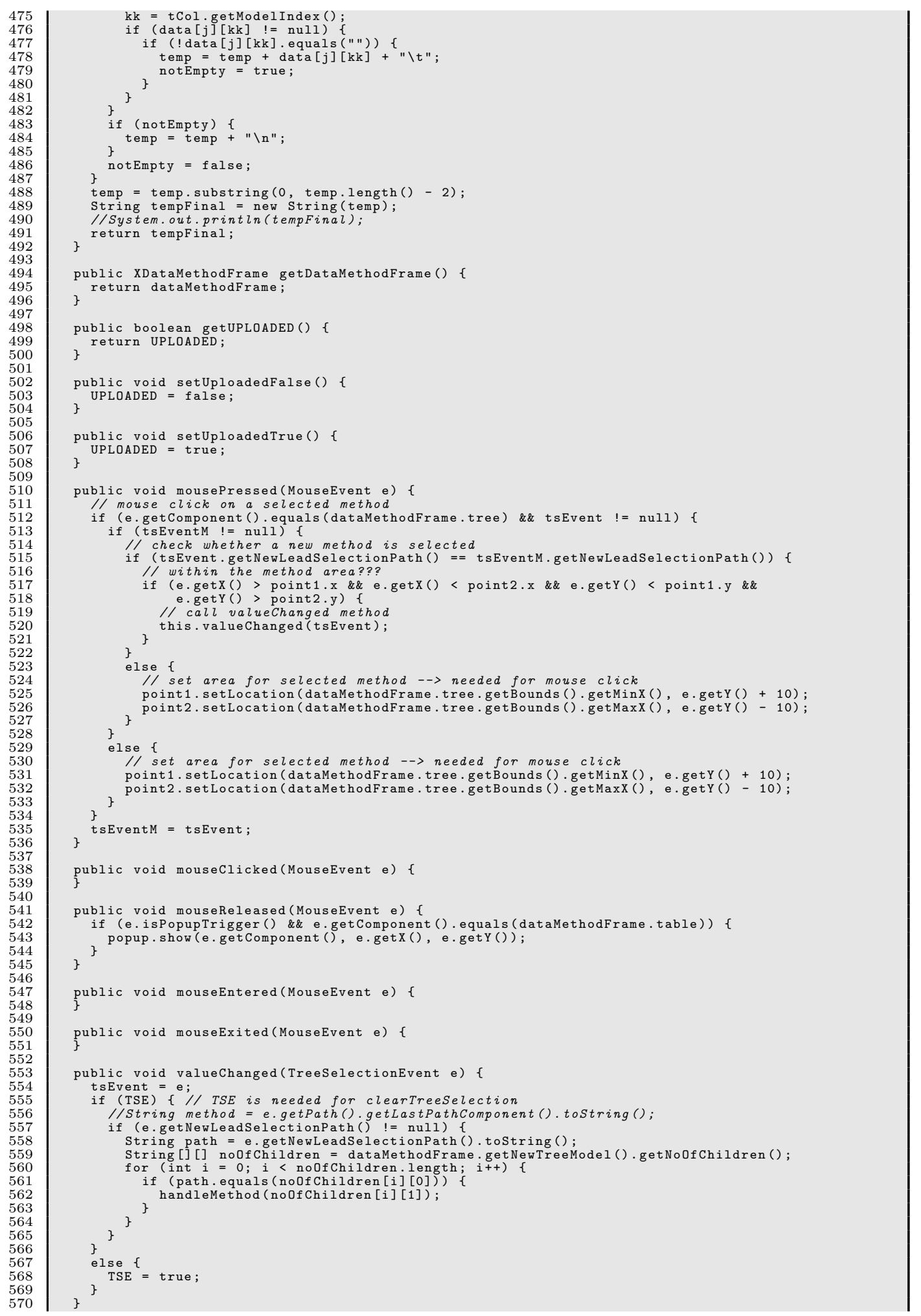




\section{XQC Source Code}

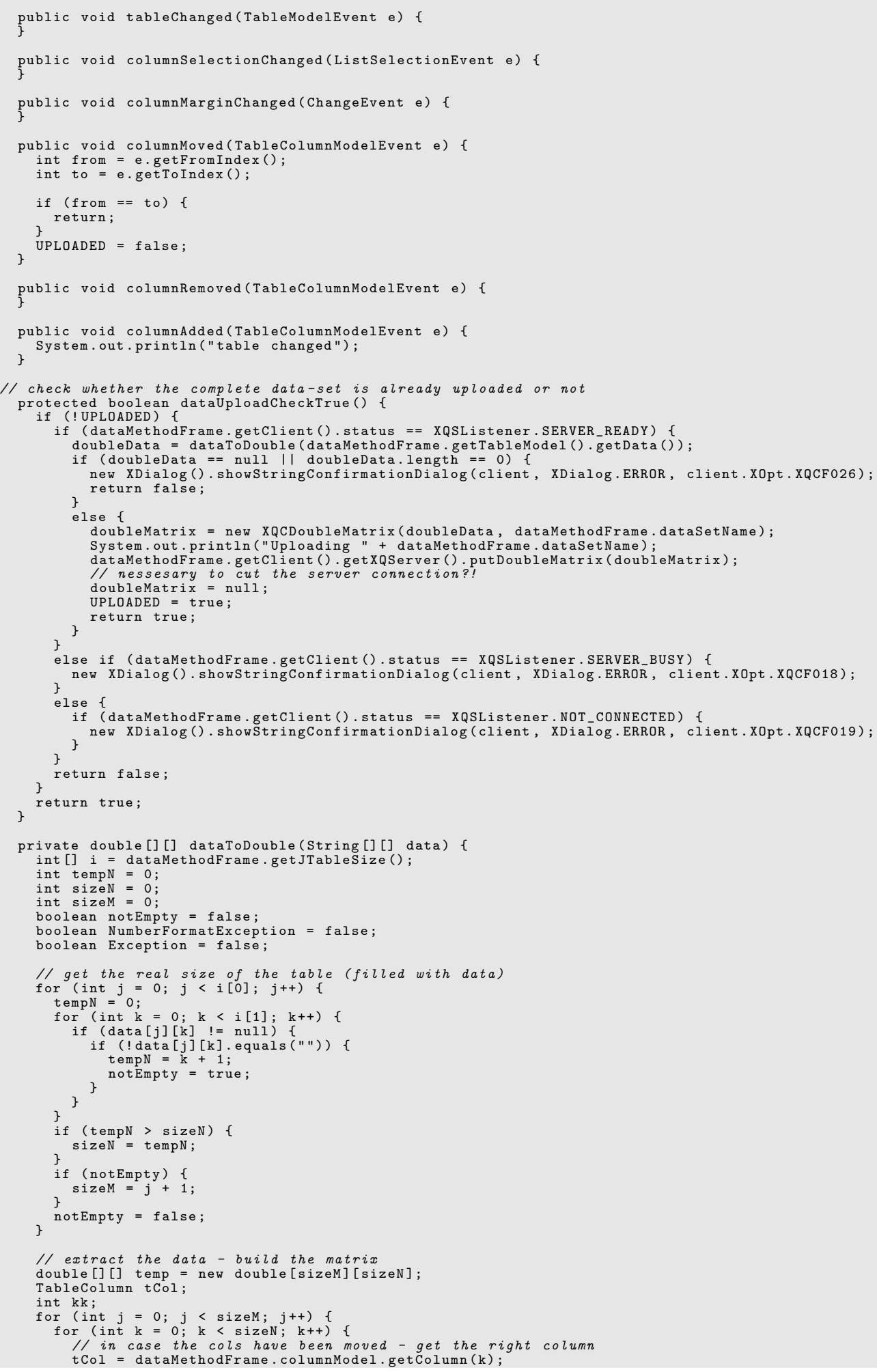




\section{B.9 XDataMethodAction.java}

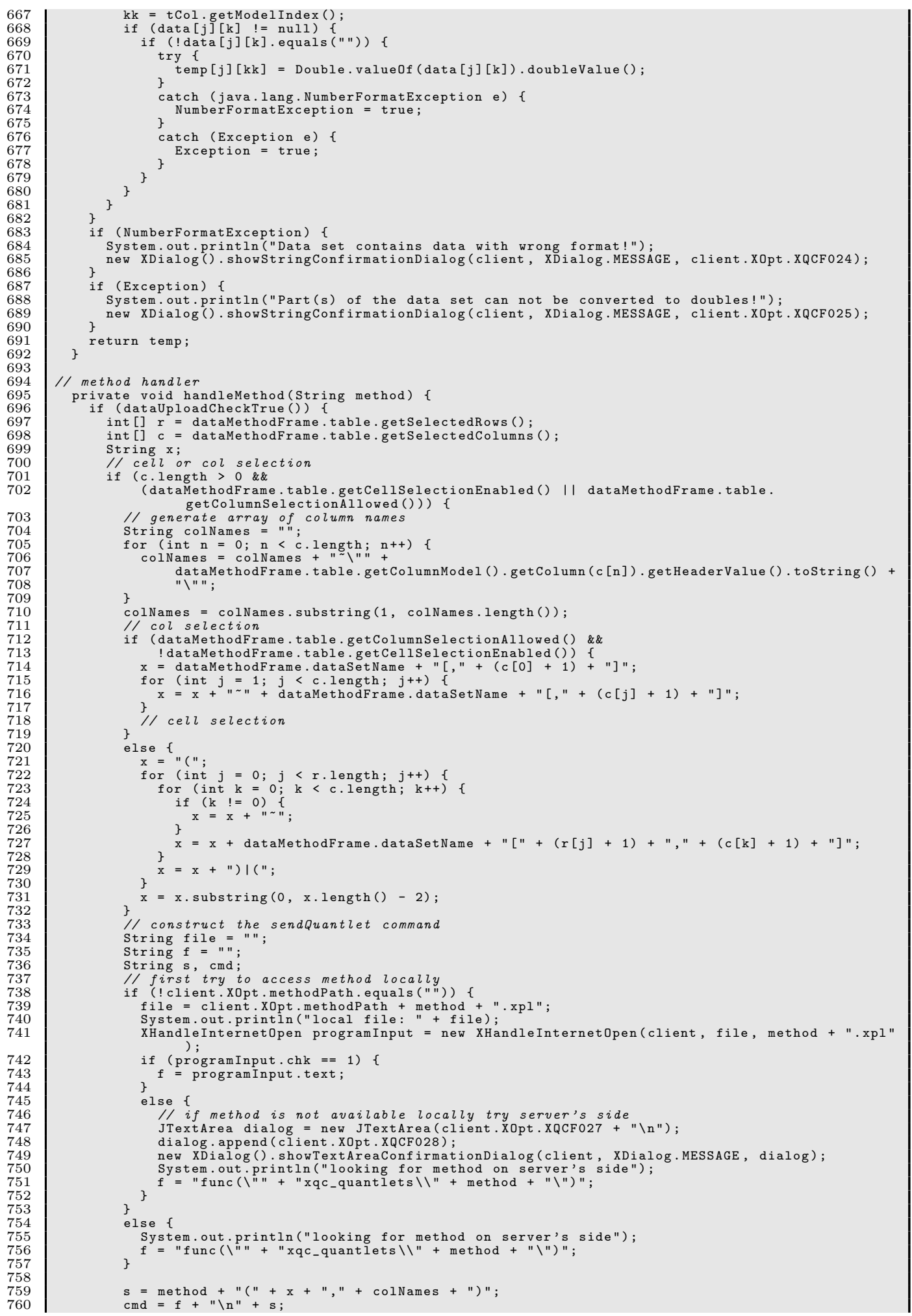




\section{XQC Source Code}

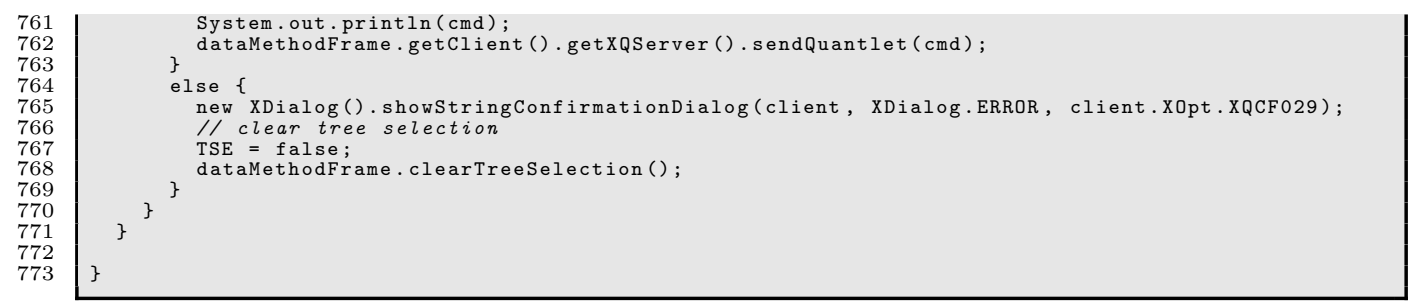




\section{B.10 XDataTableModel.java}

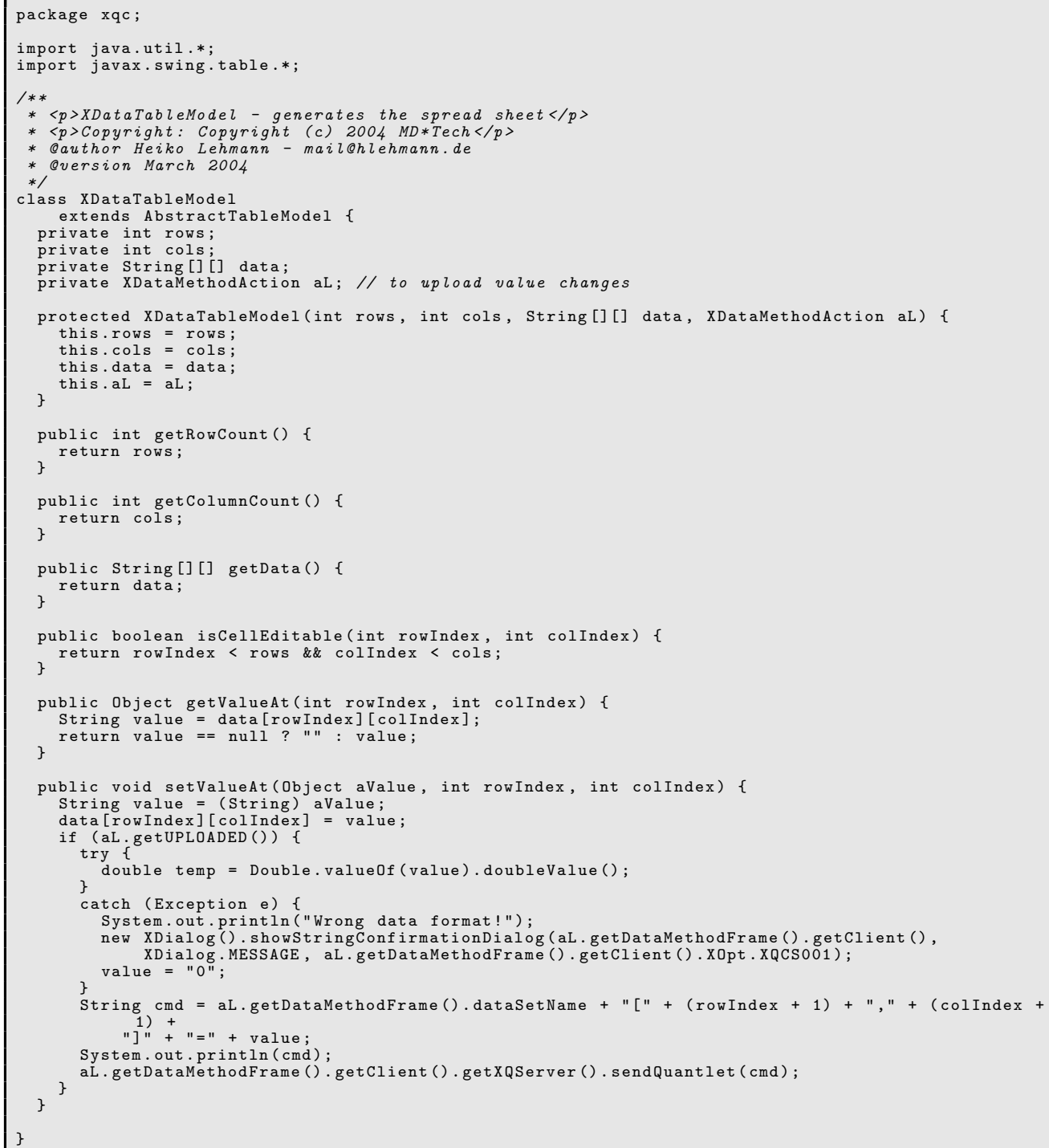




\section{B.11 XDataMethodFrameTree.java}

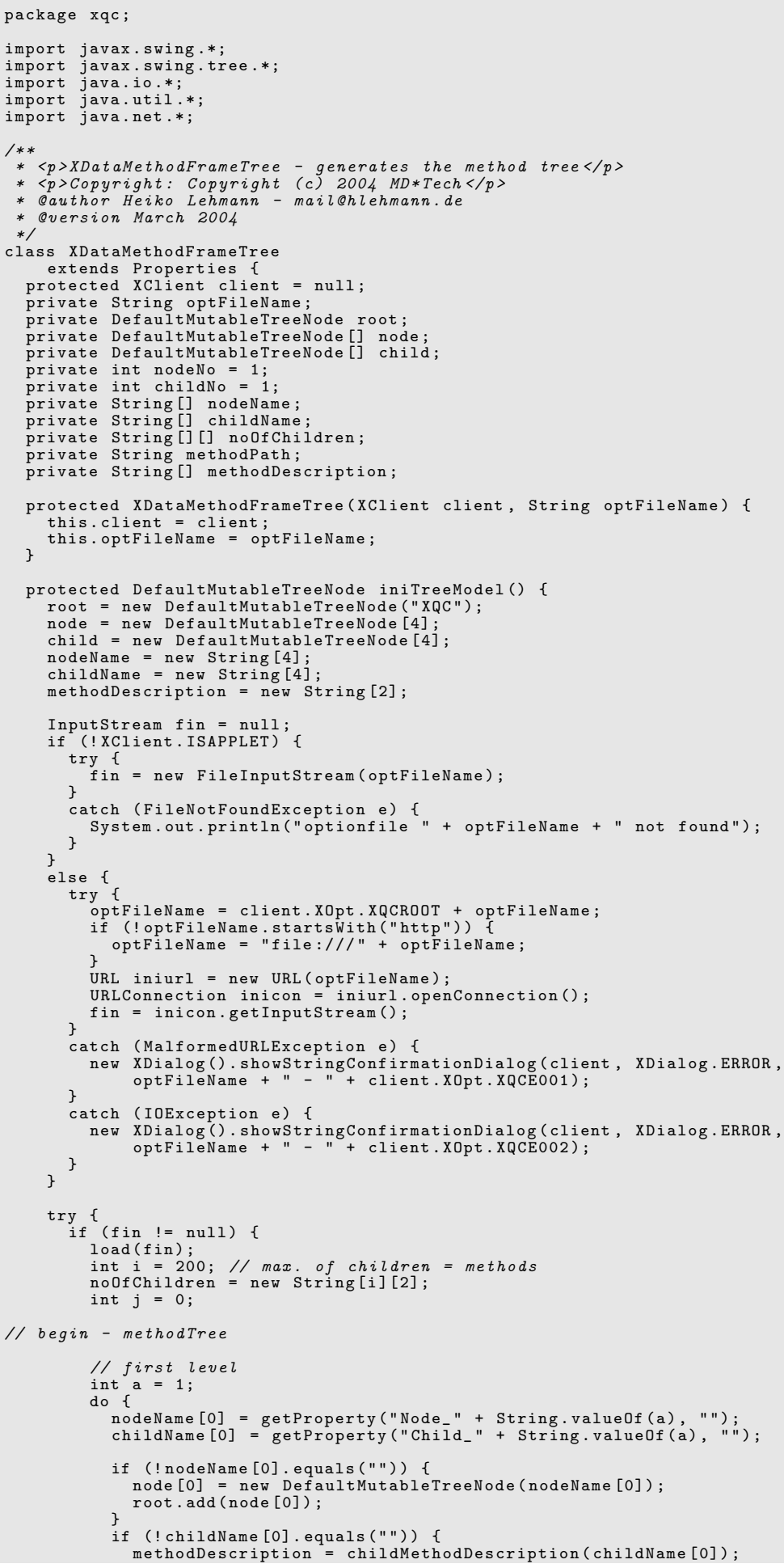




\section{B.11 XDataMethodFrameTree.java}

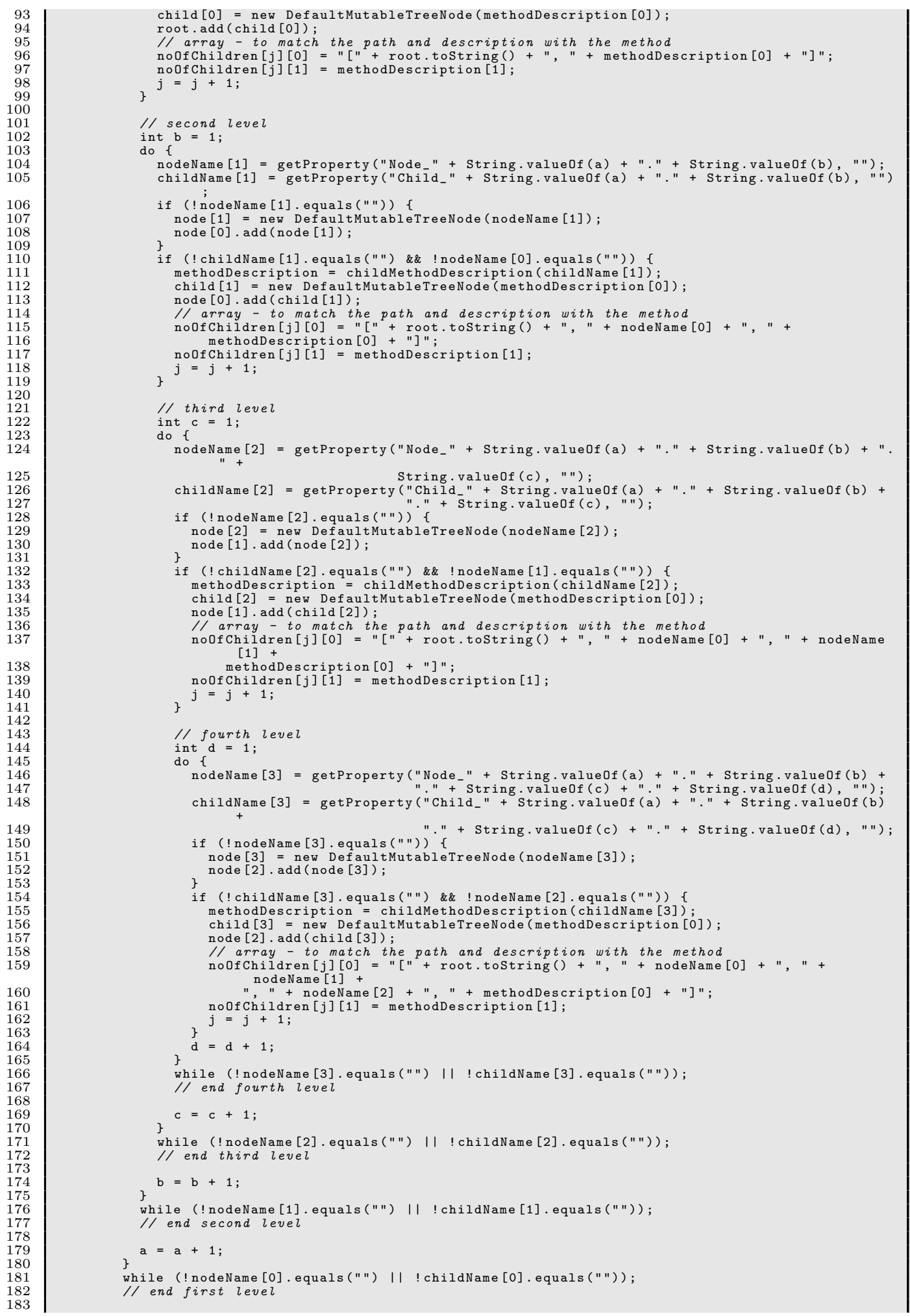




\section{XQC Source Code}

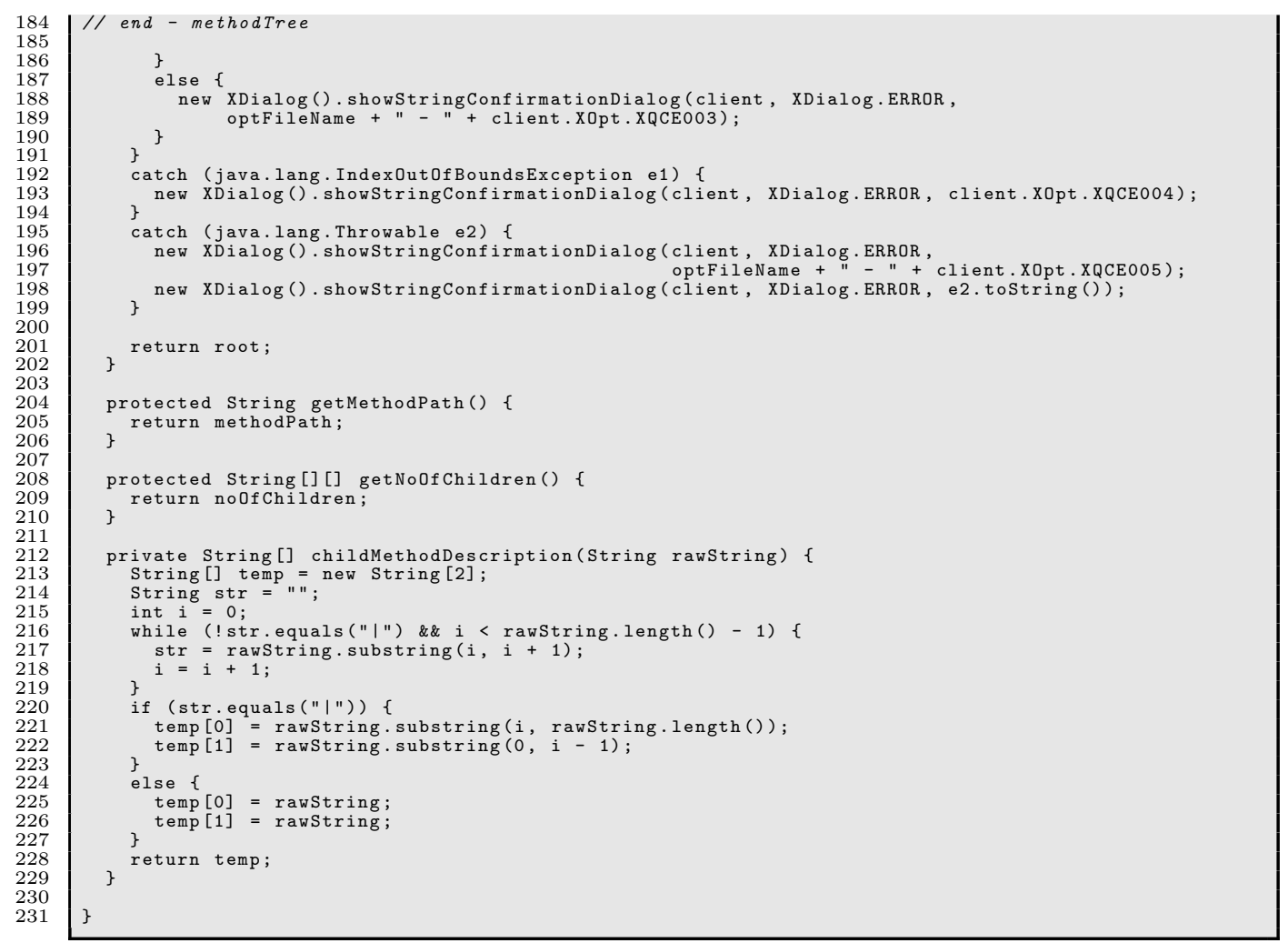


B.12 XDisplayFrame.java

\section{B.12 XDisplayFrame.java}

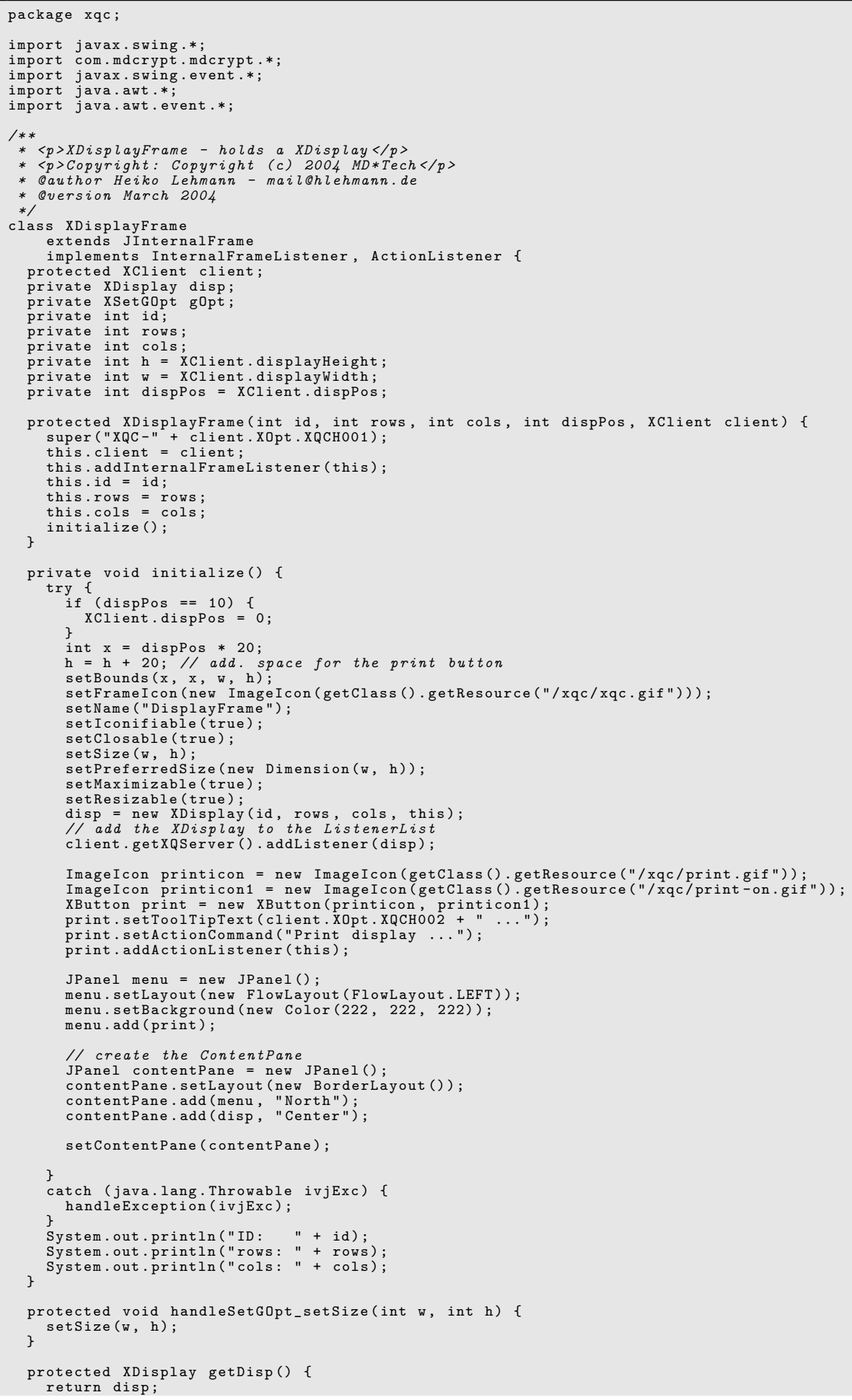




\section{XQC Source Code}

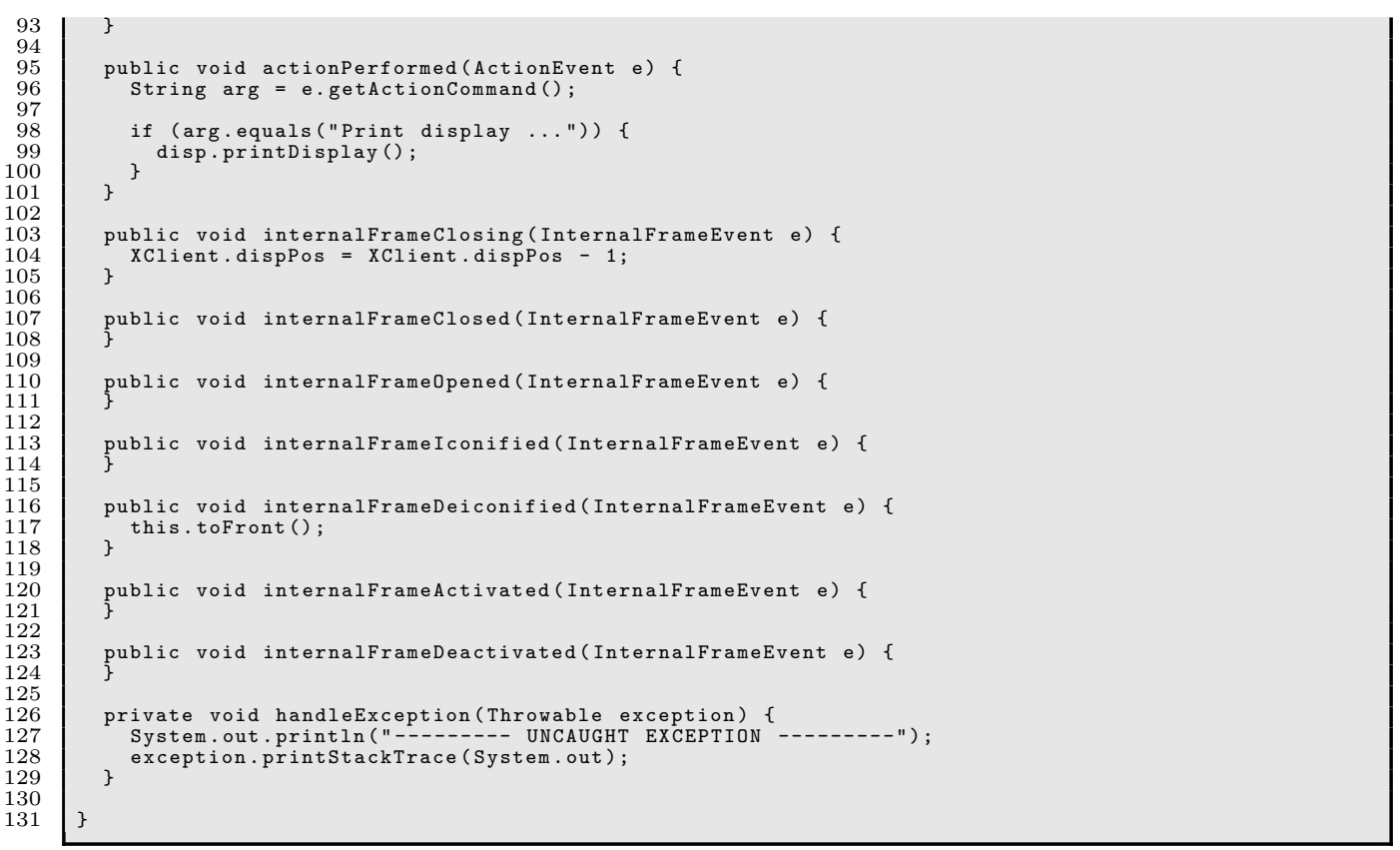




\section{B.13 XDisplay.java}

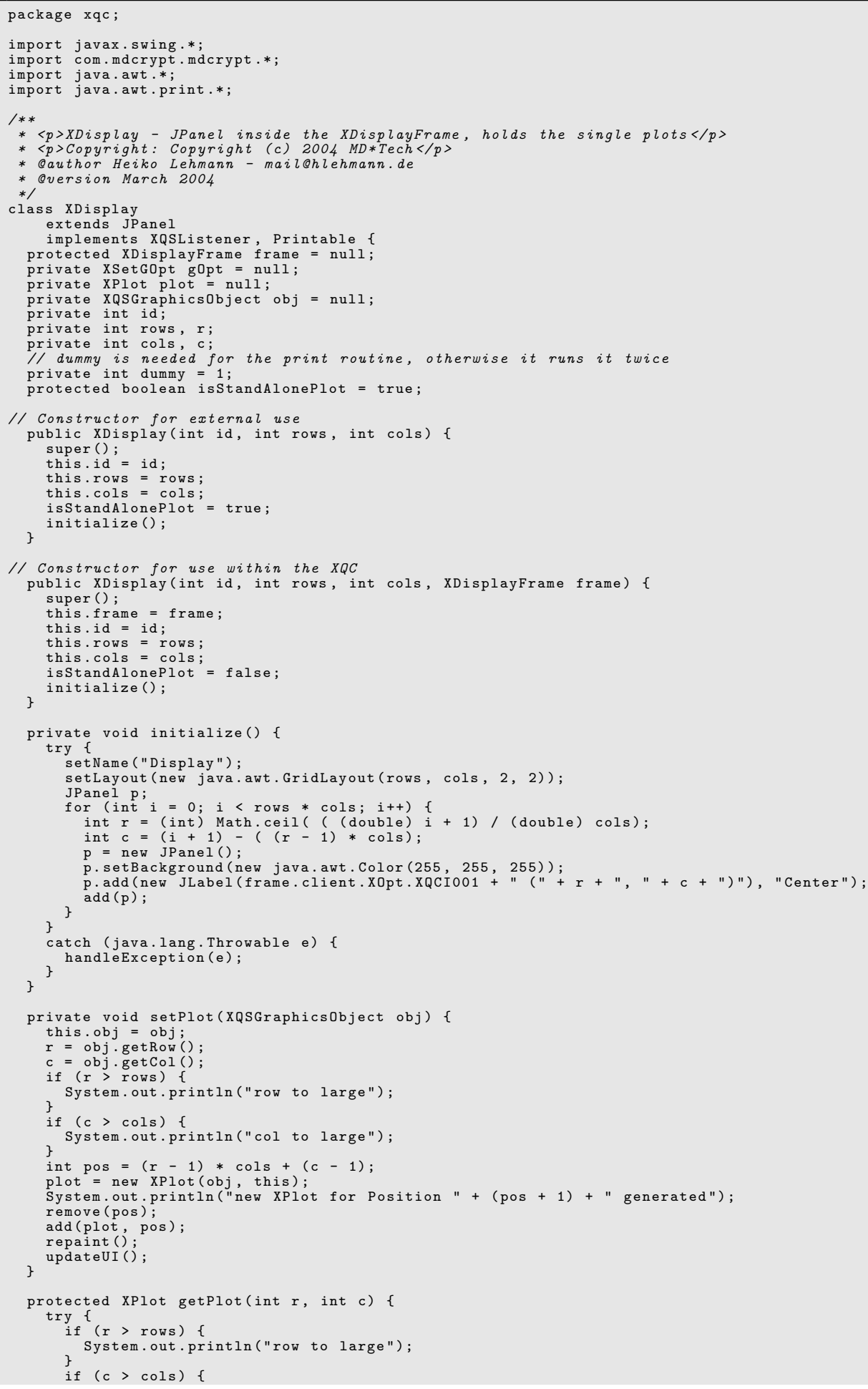




\section{XQC Source Code}

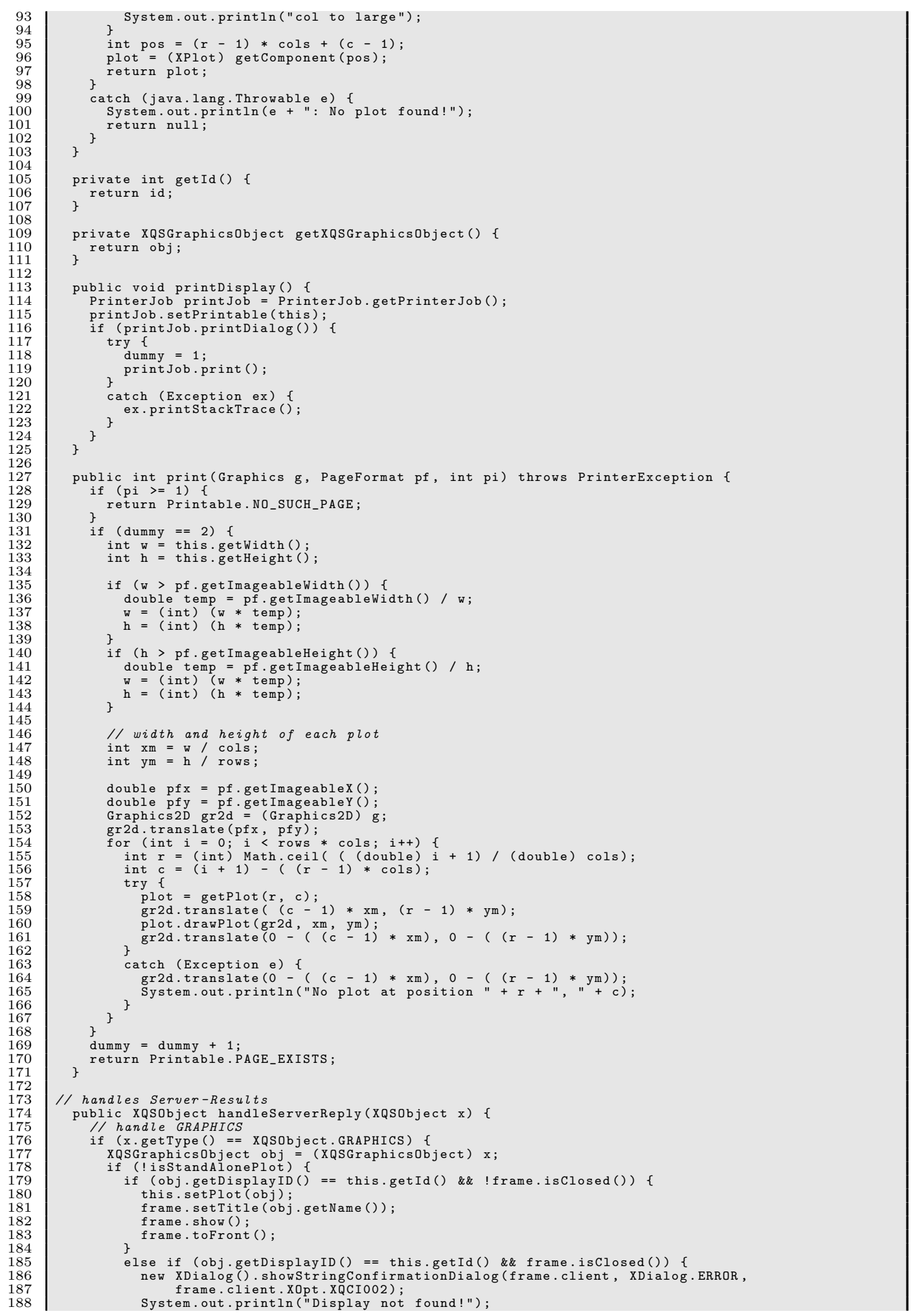




\section{B.13 XDisplay.java}

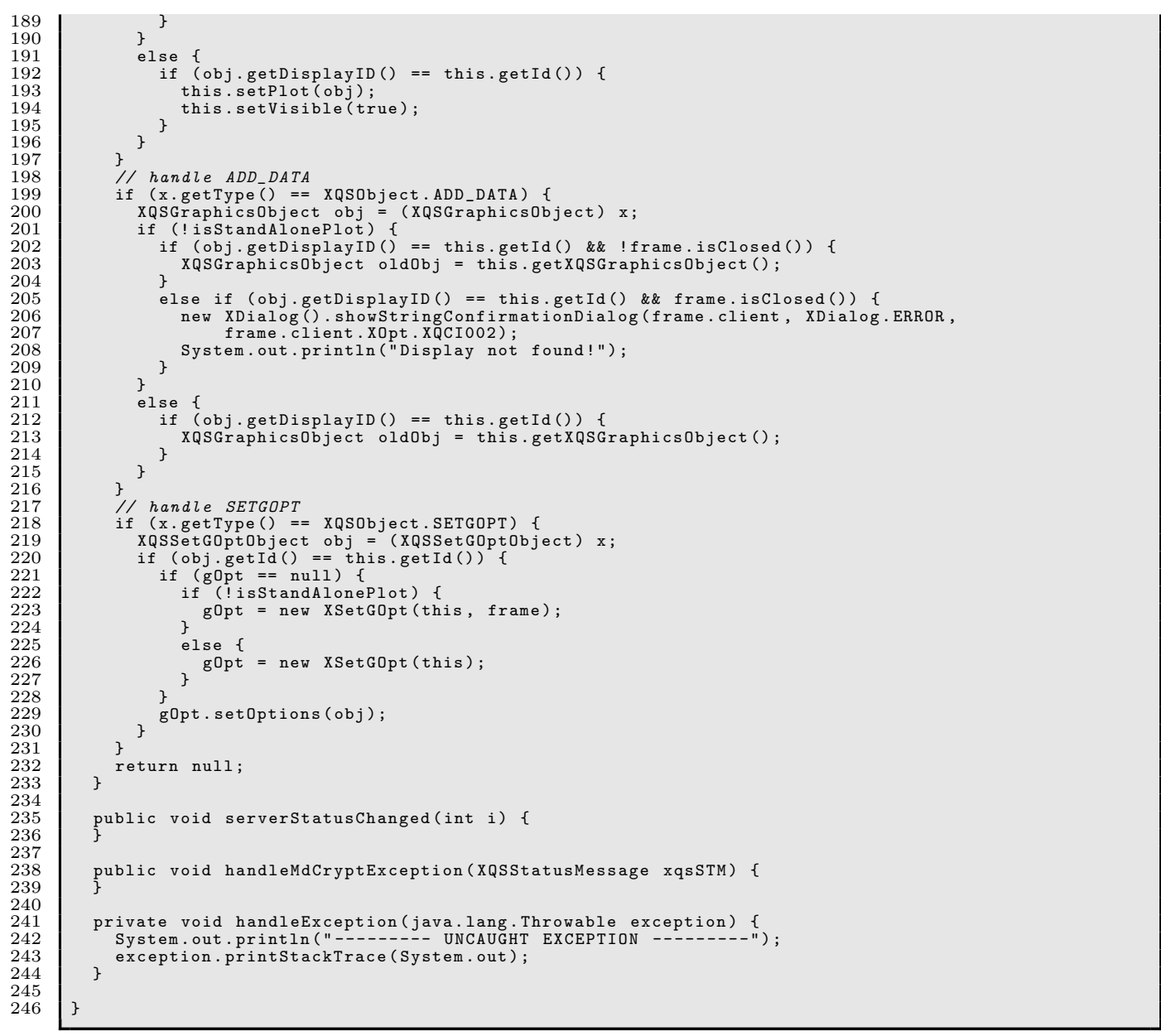




\section{B.14 XPlot.java}

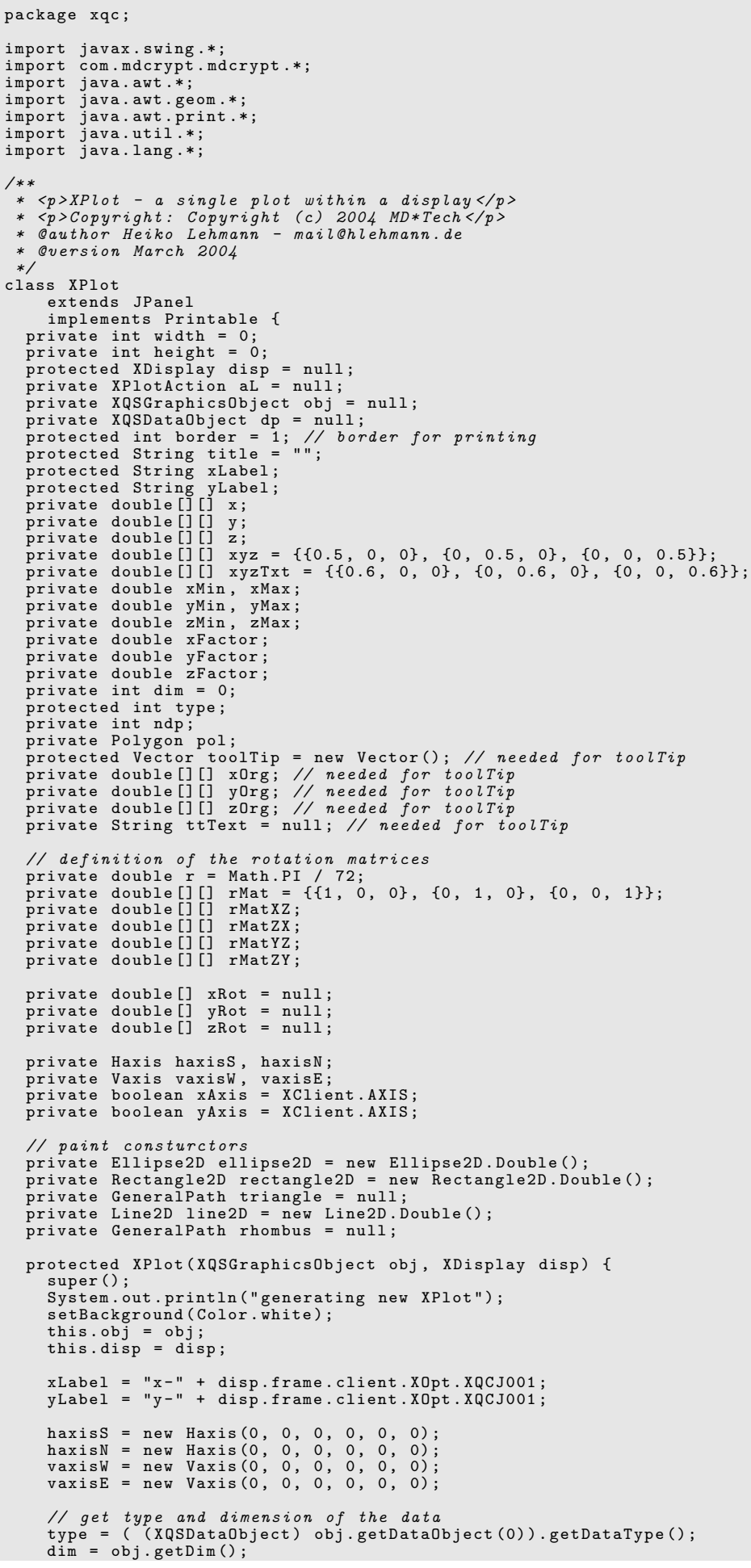




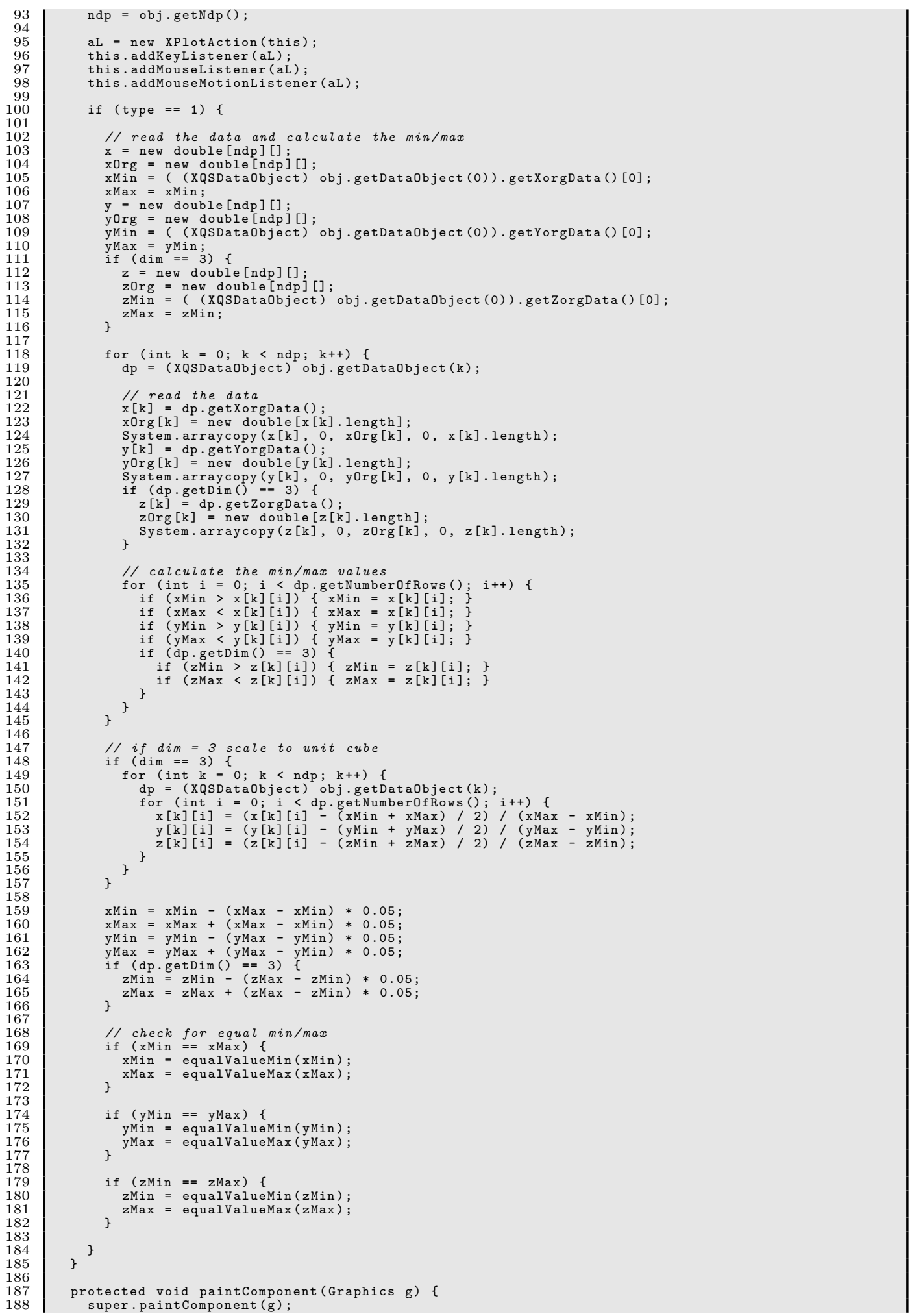




\section{XQC Source Code}

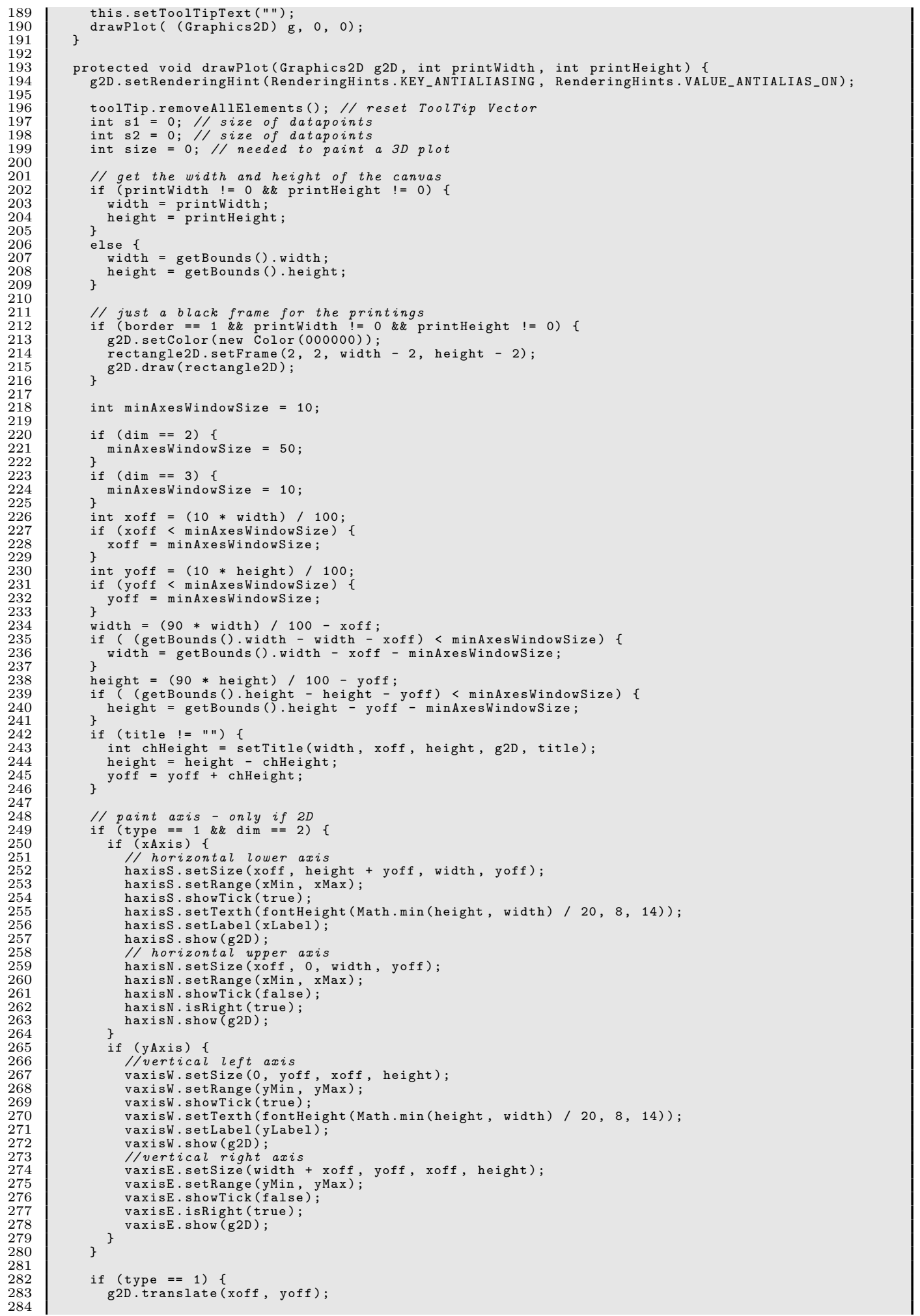




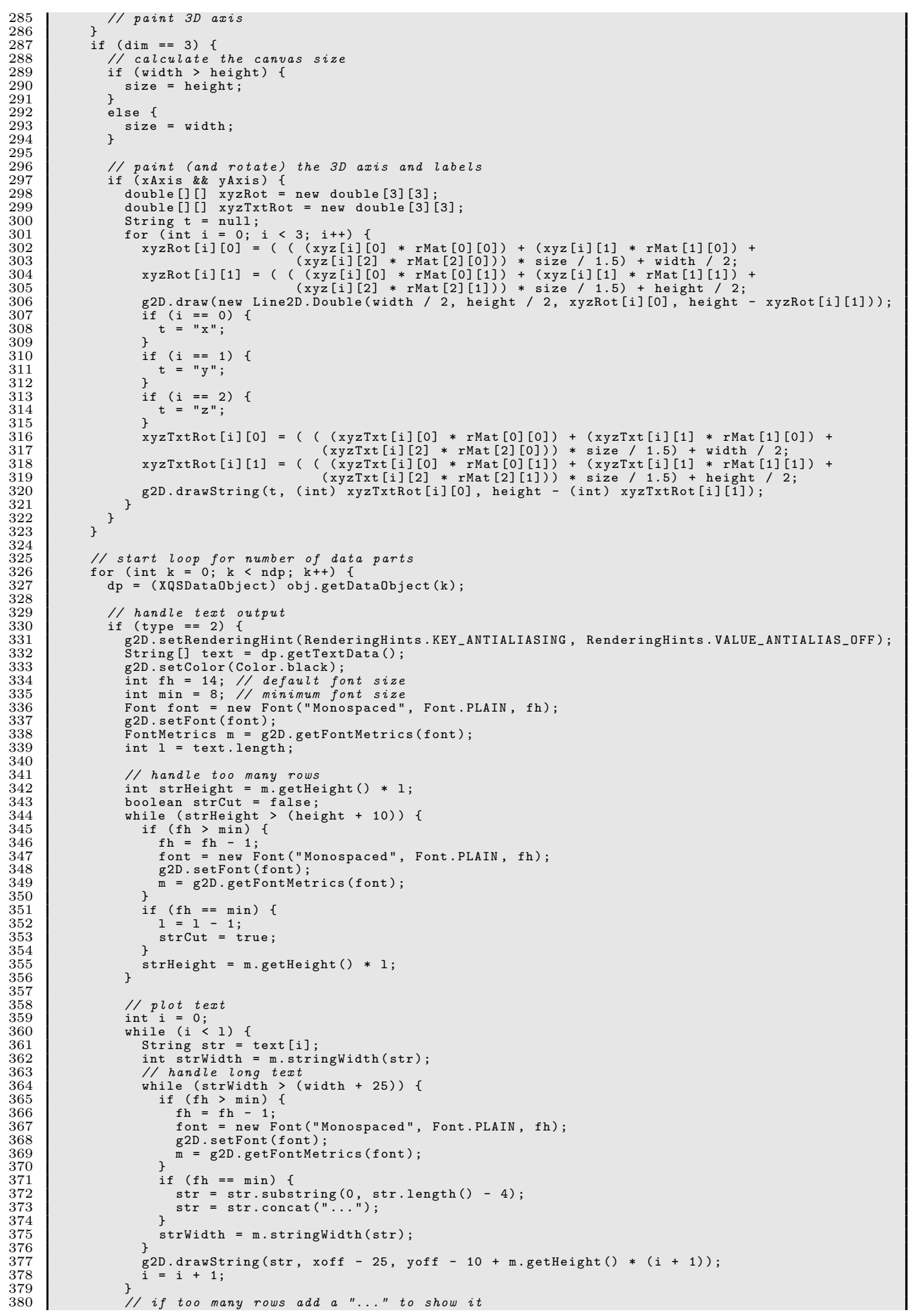




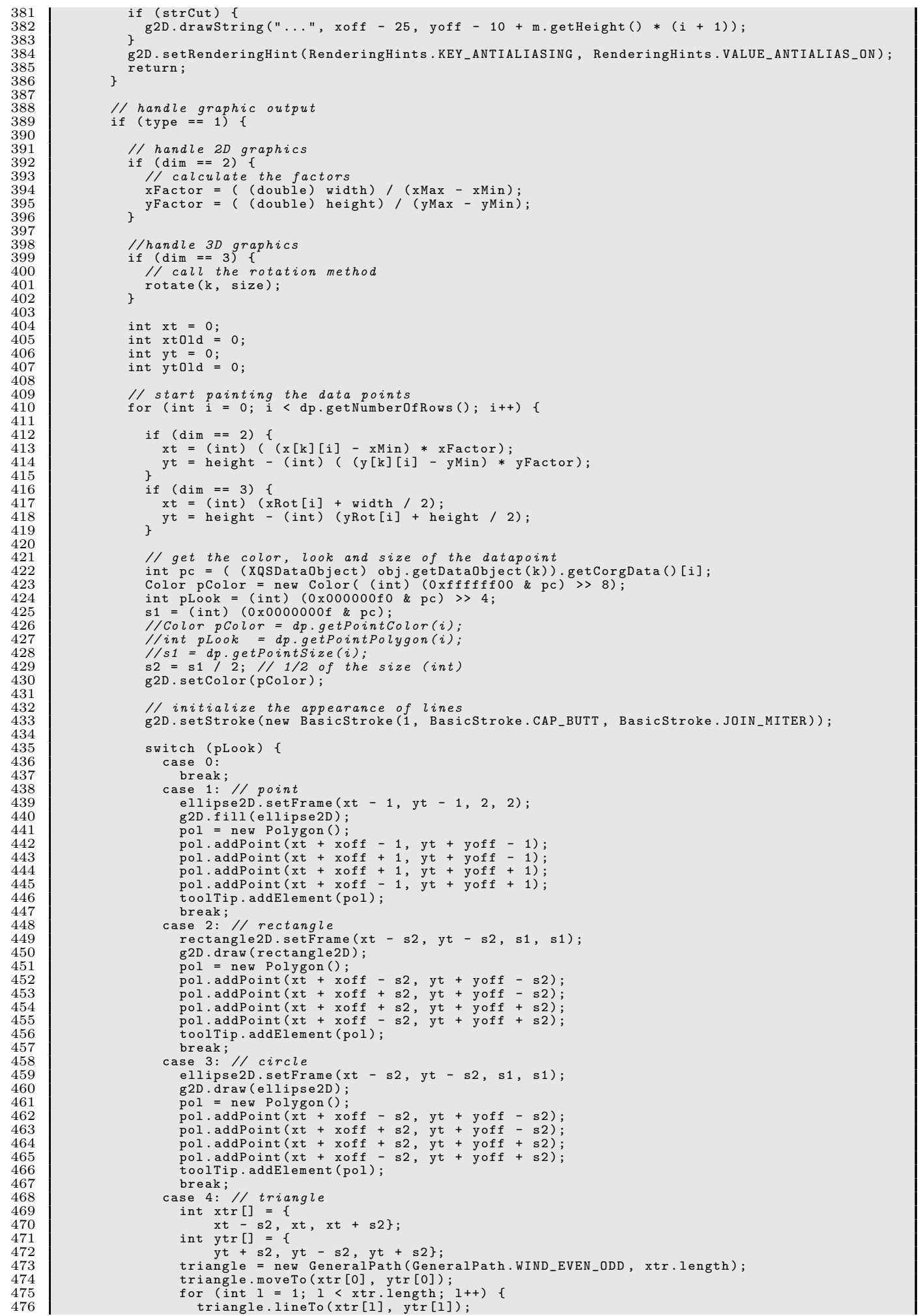




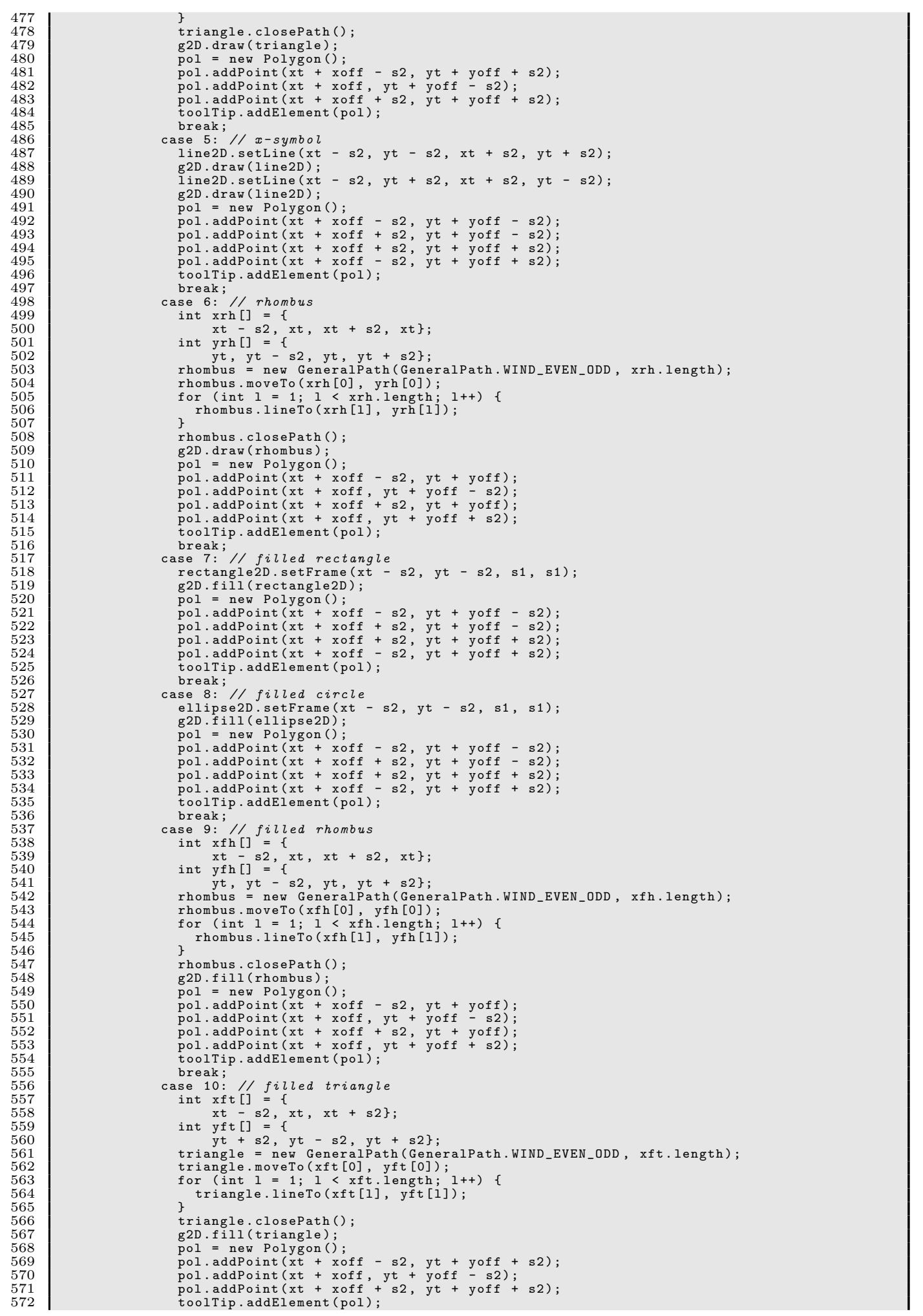




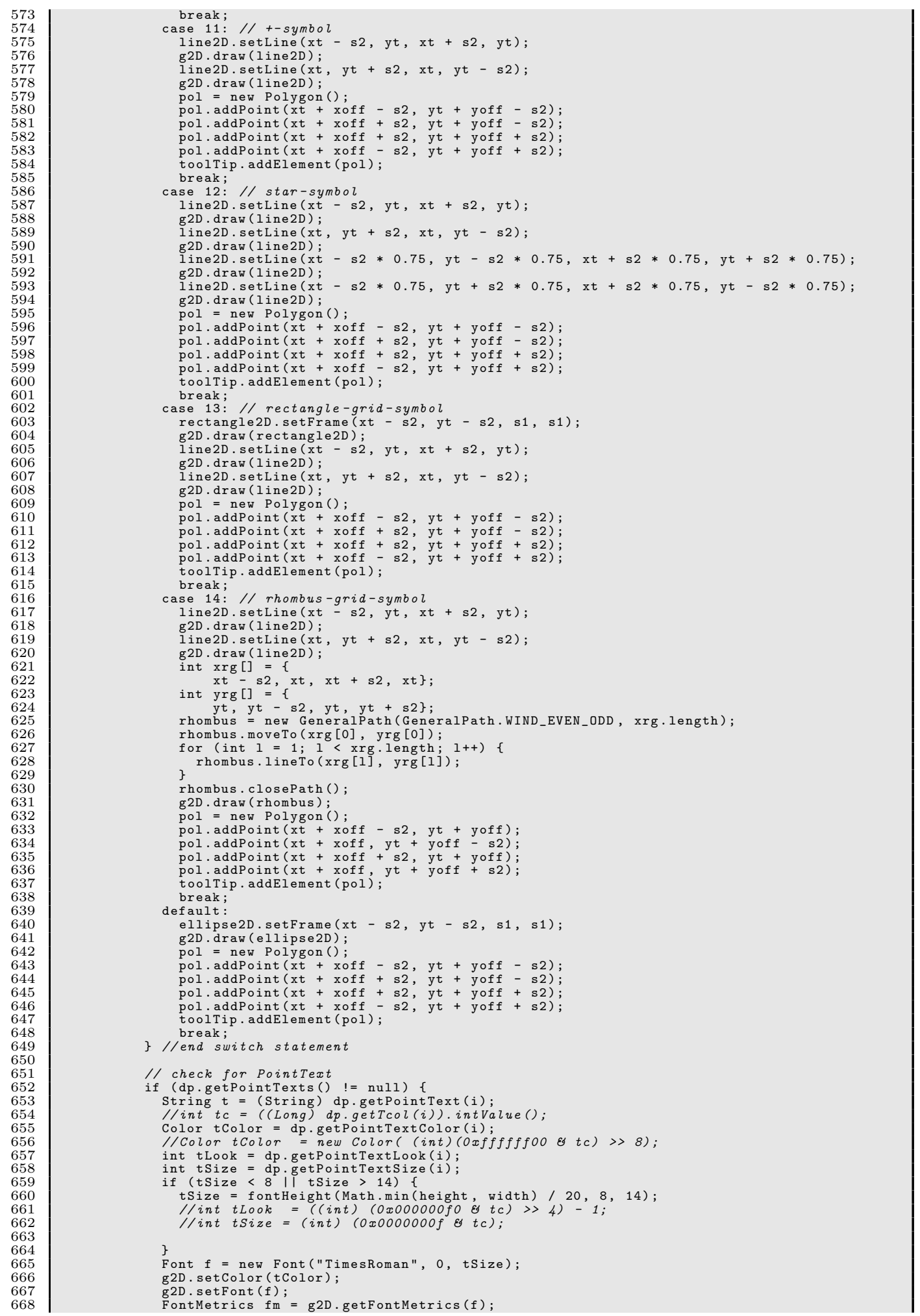




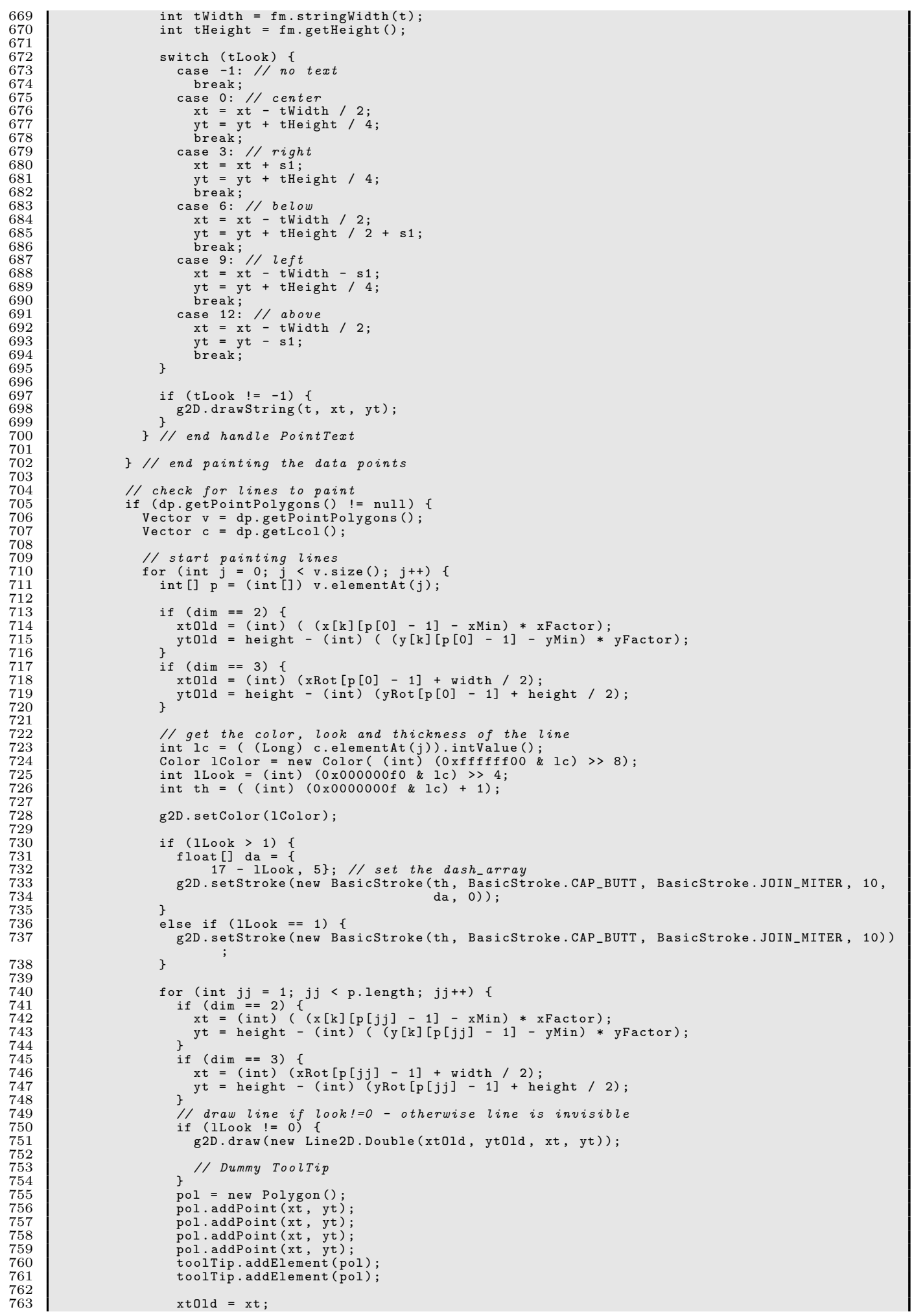




\section{XQC Source Code}

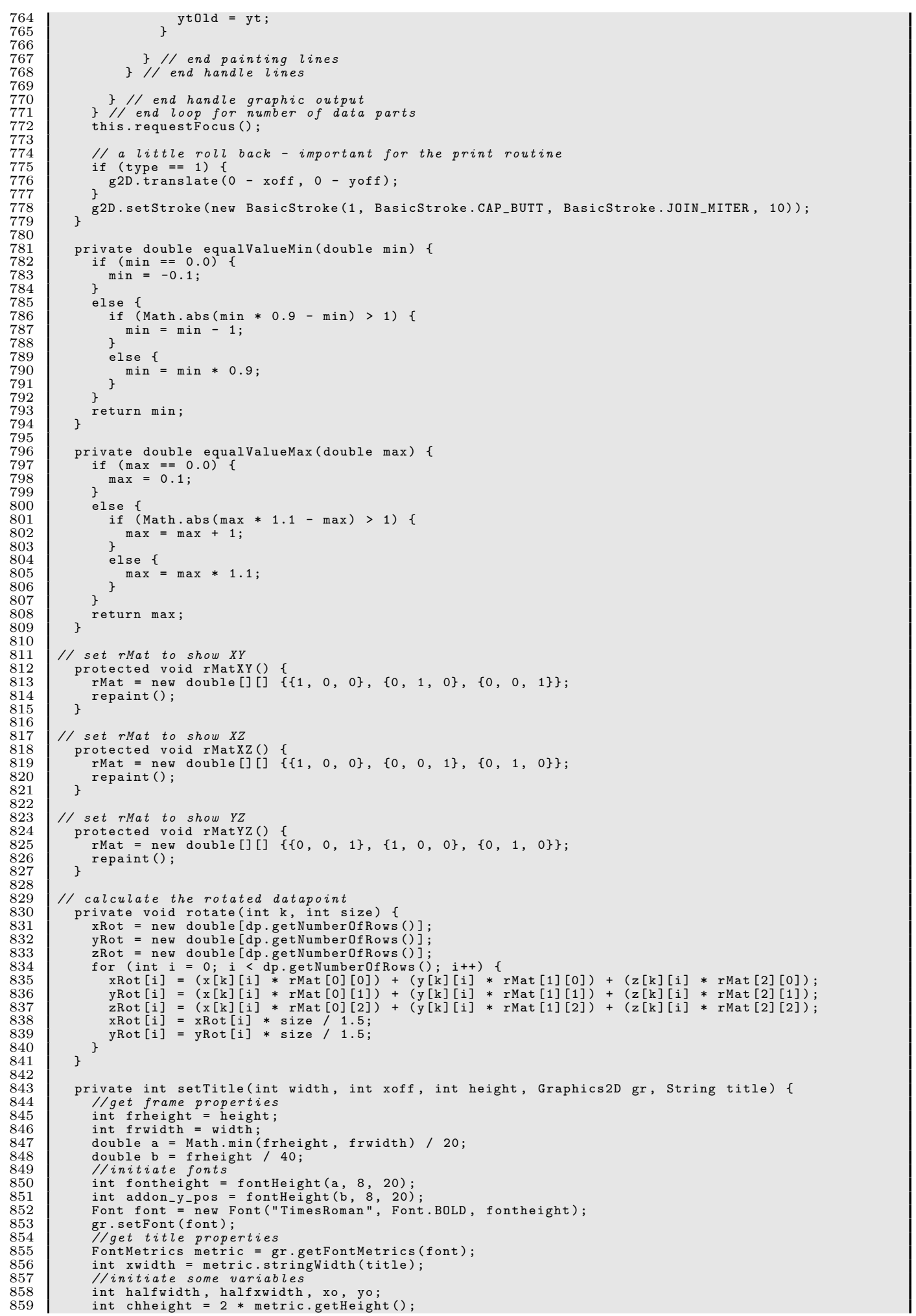




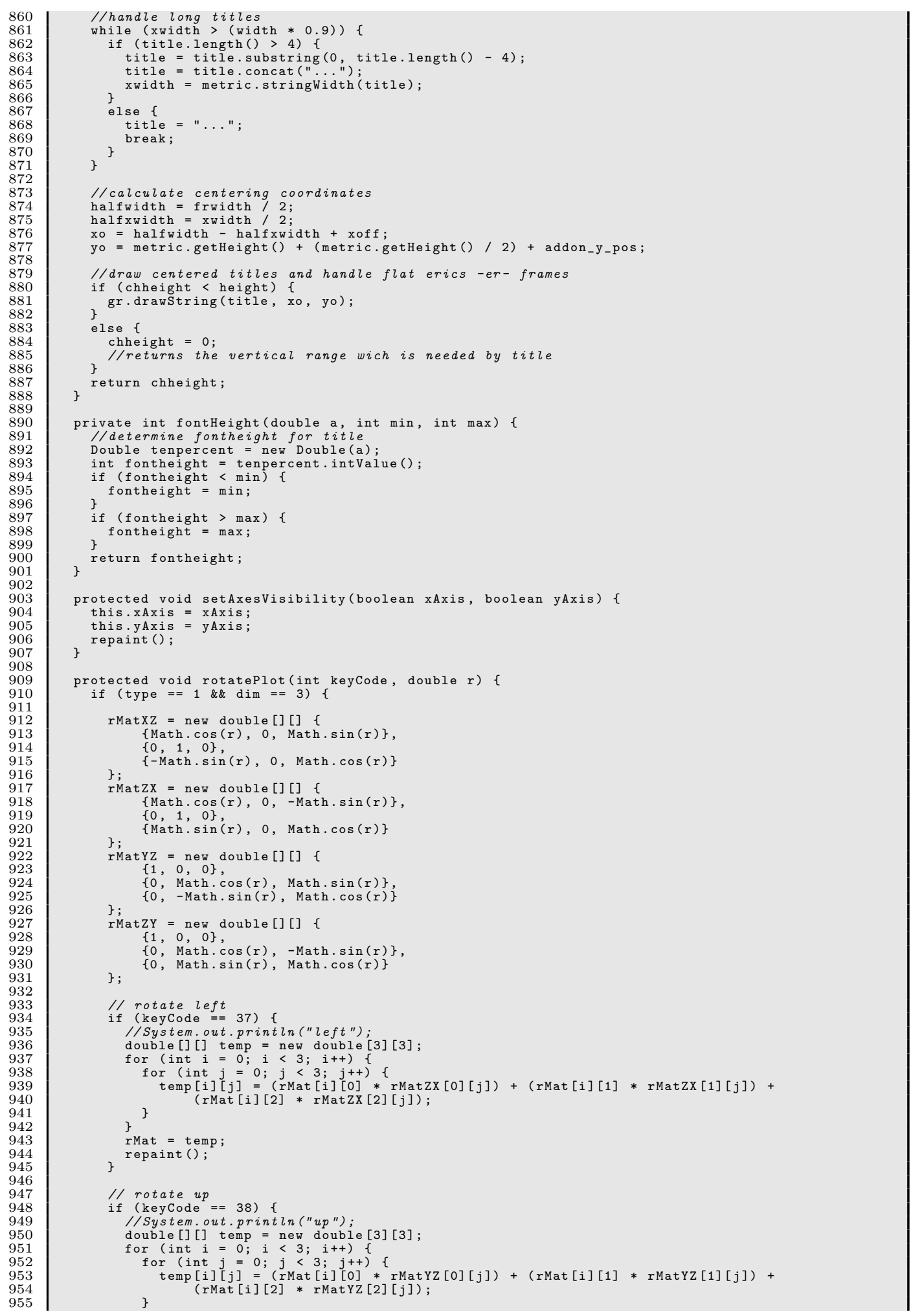




\section{XQC Source Code}

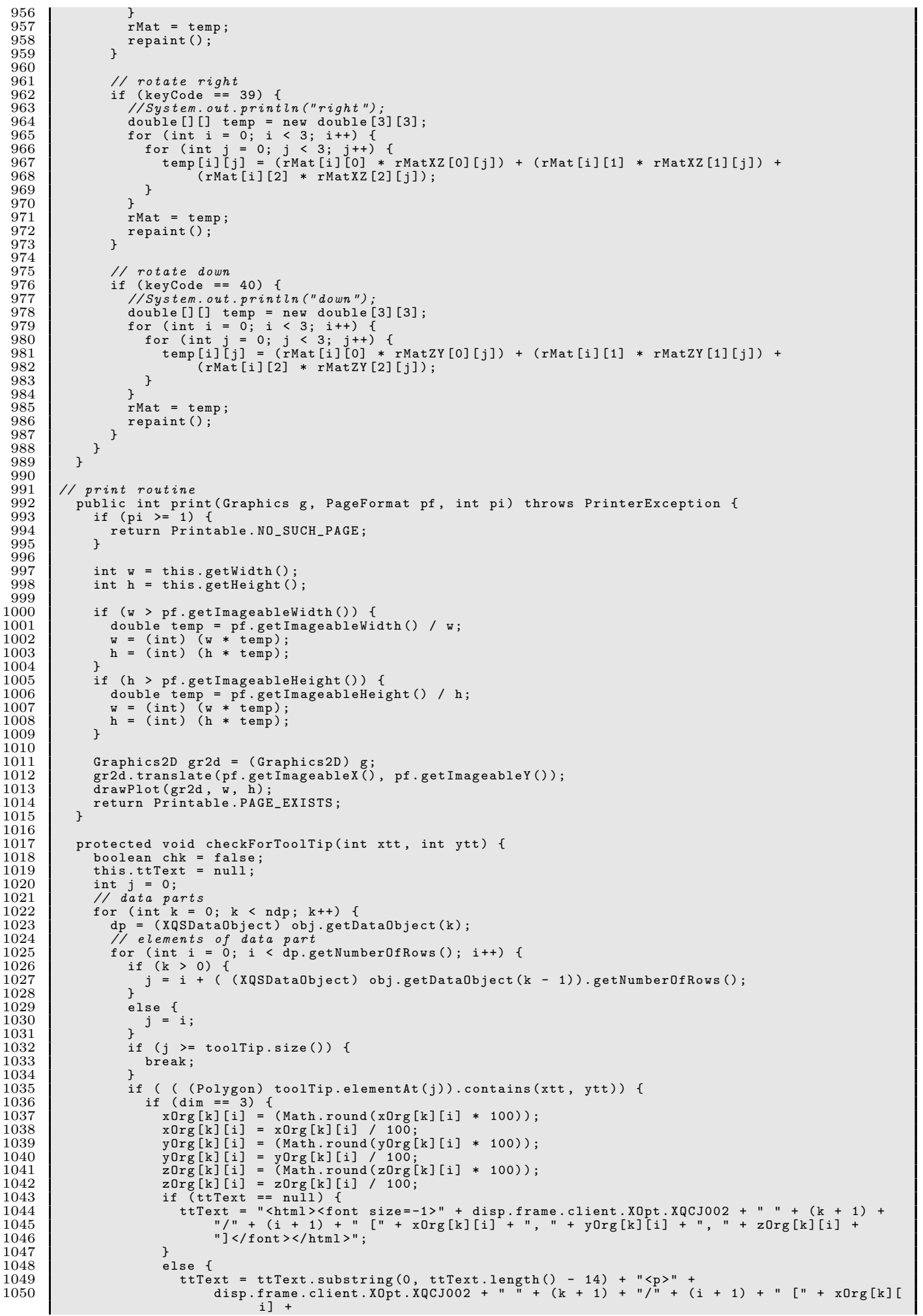




\section{B.14 XPlot.java}

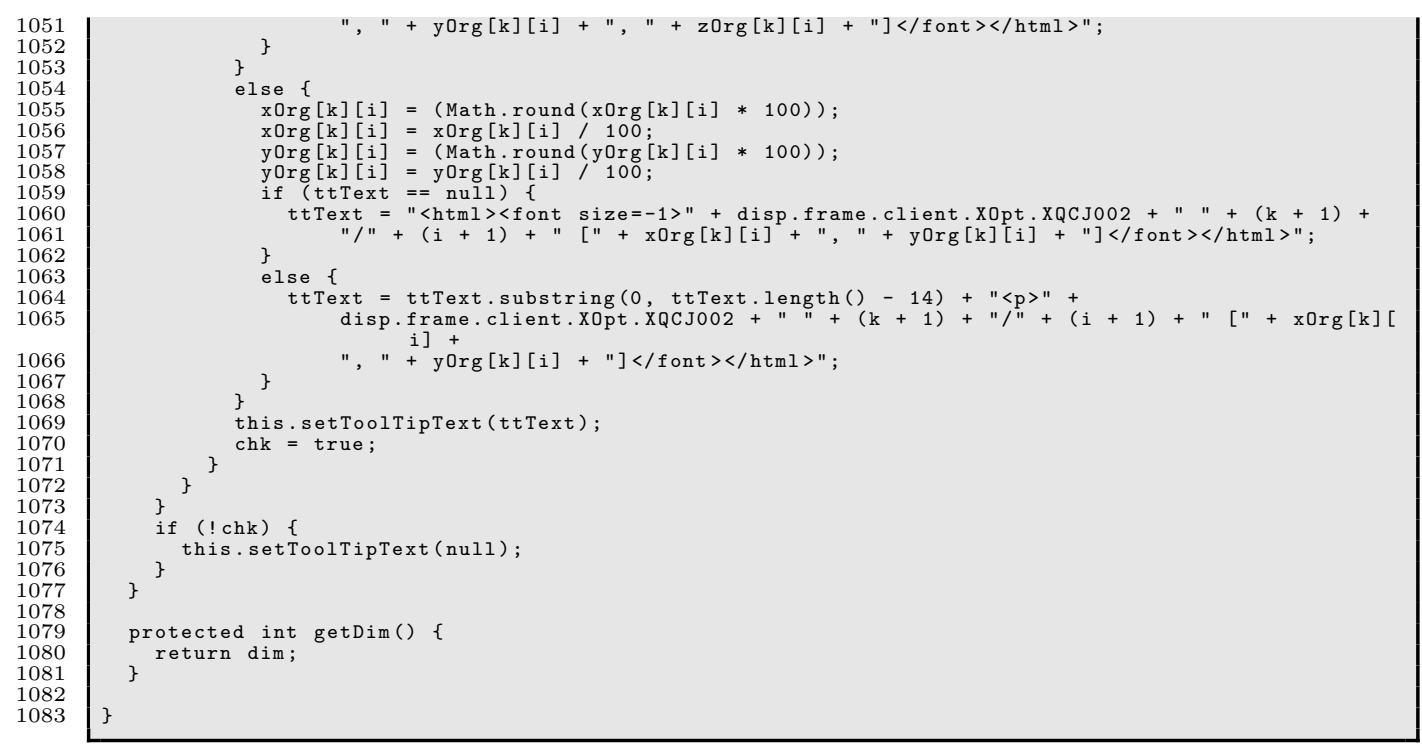




\section{B.15 XPlotAction.java}

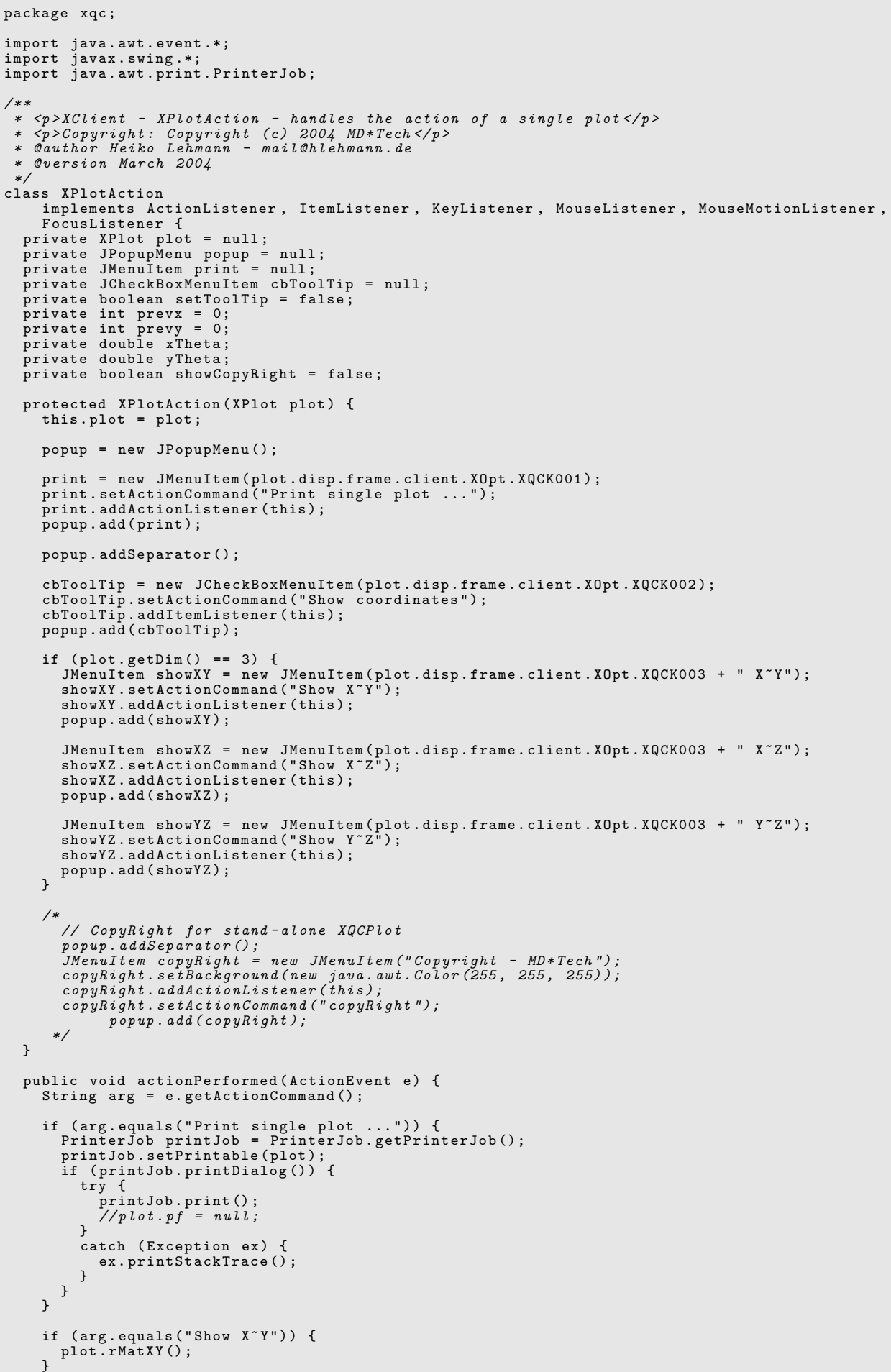




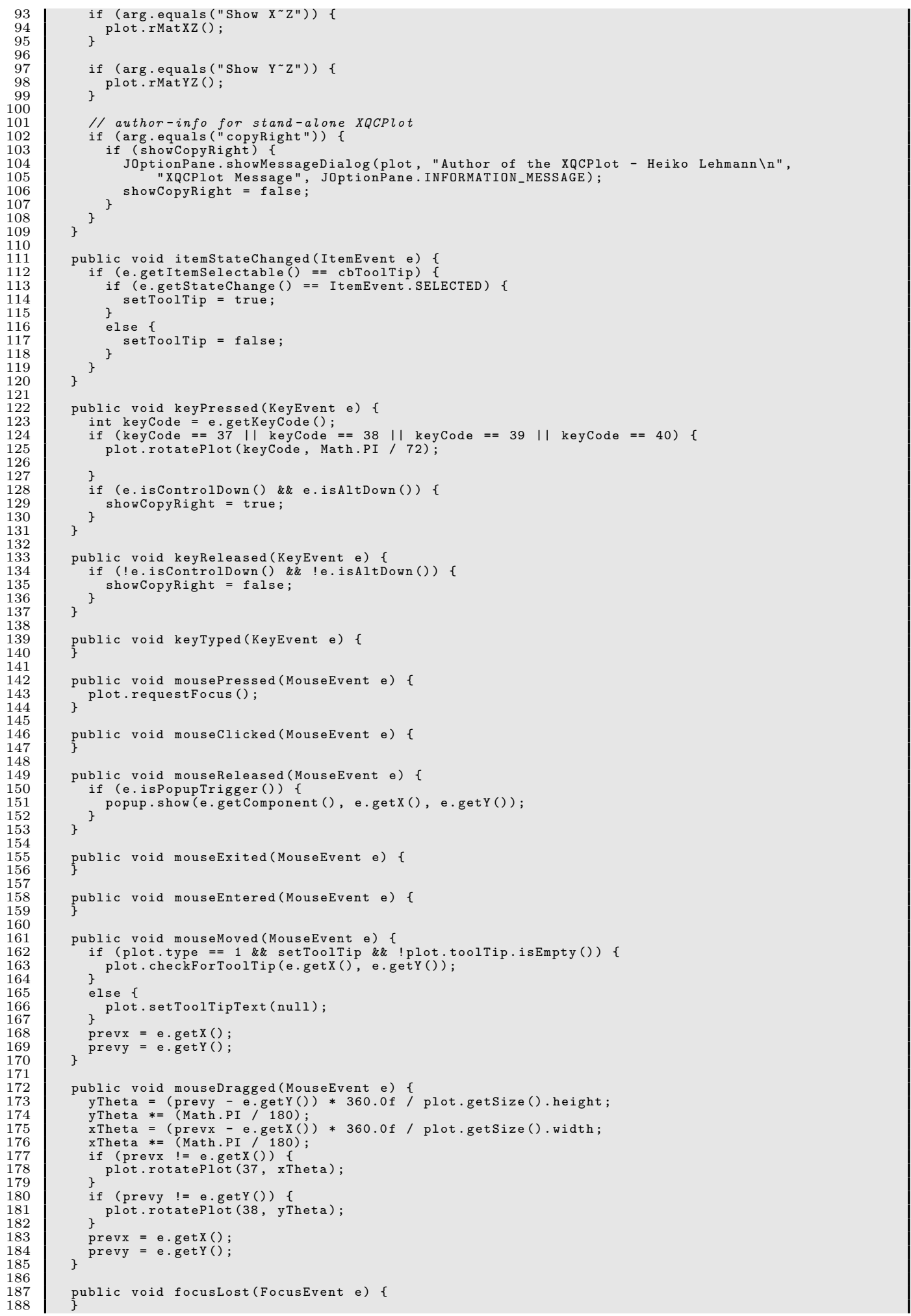




\section{XQC Source Code}

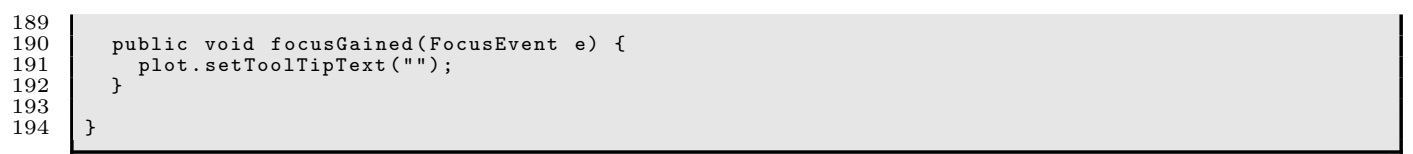




\section{B.16 XSetGOpt.java}

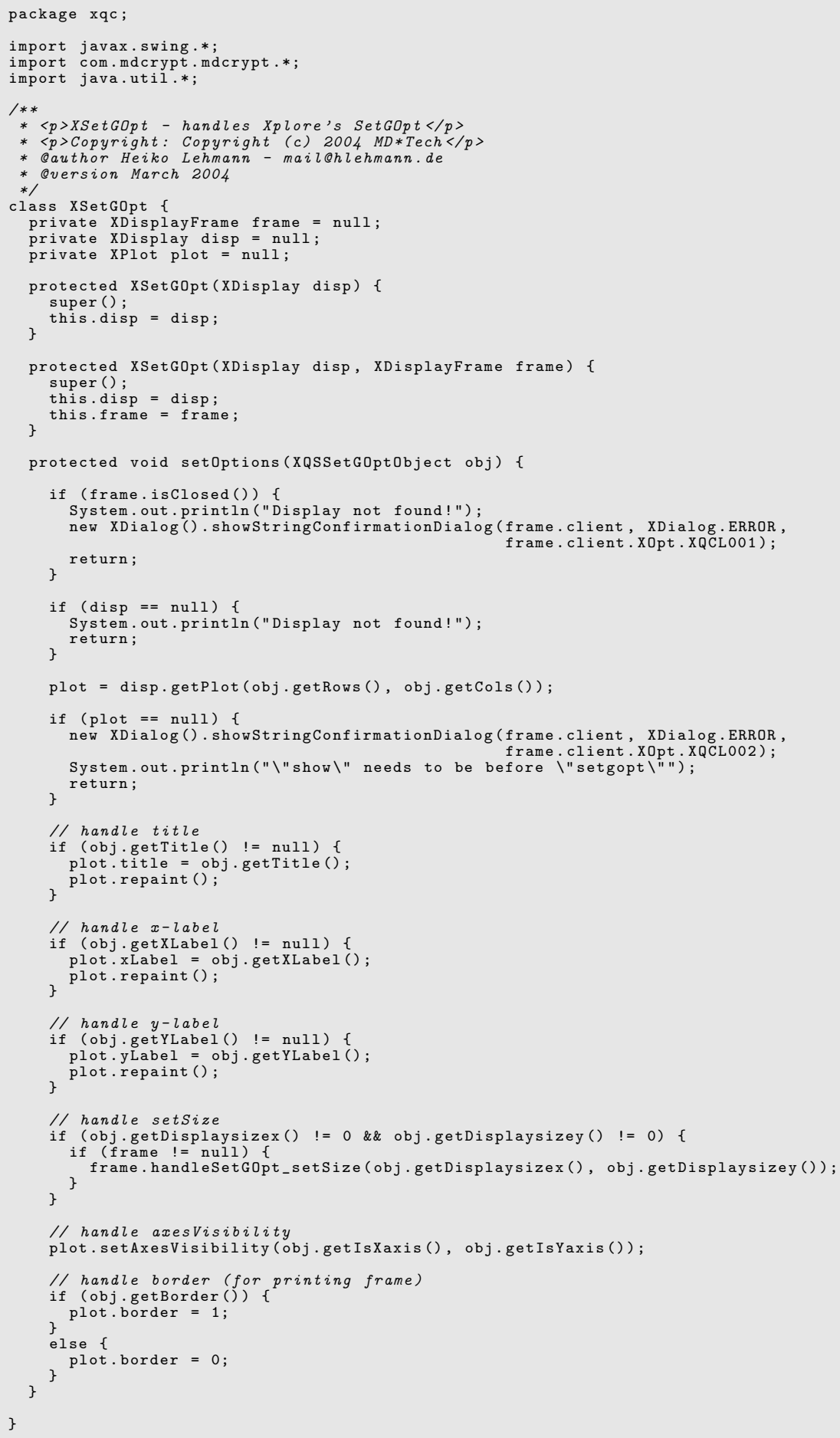




\section{B.17 XReadValue.java}

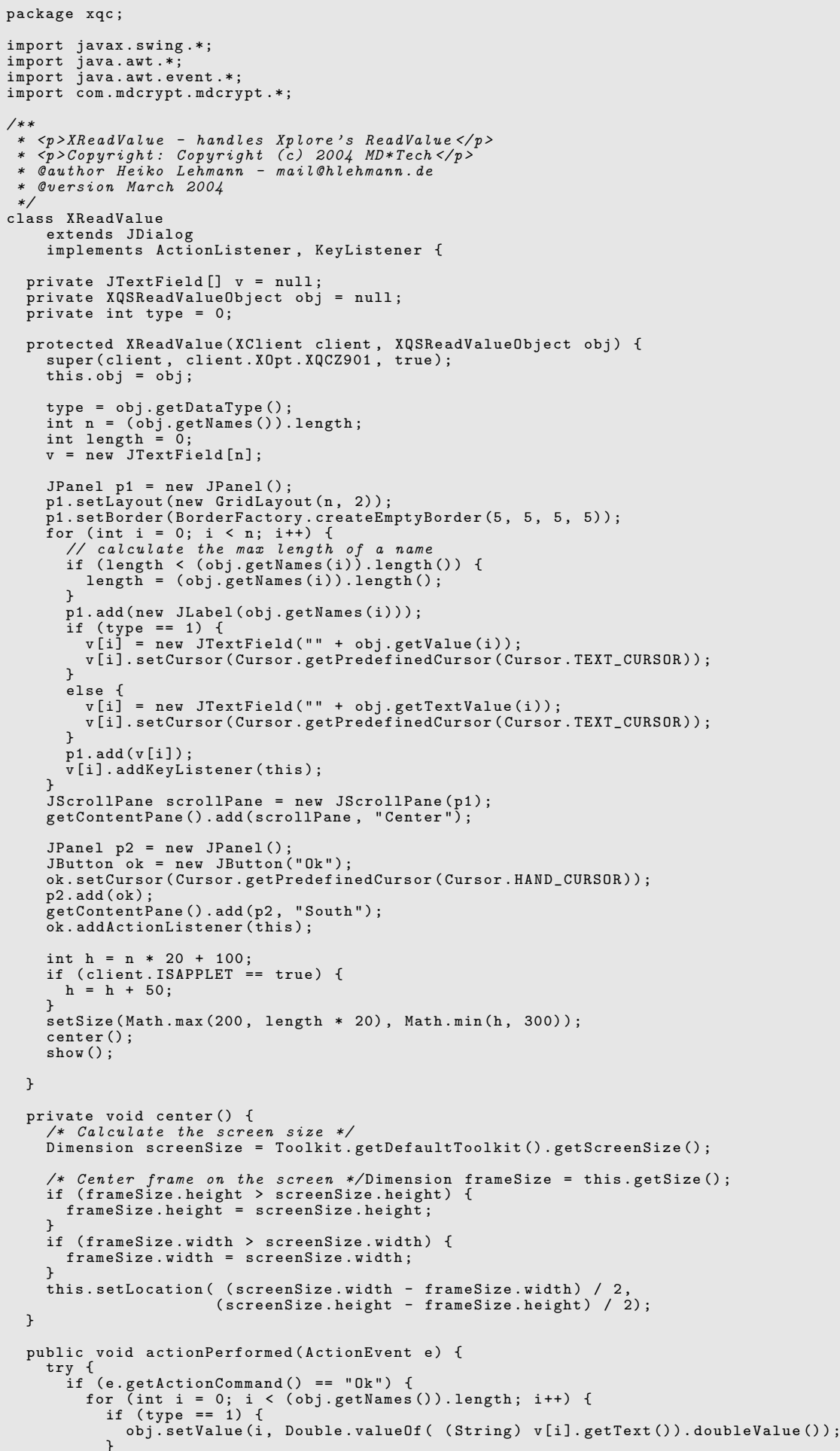




\section{B.17 XReadValue.java}

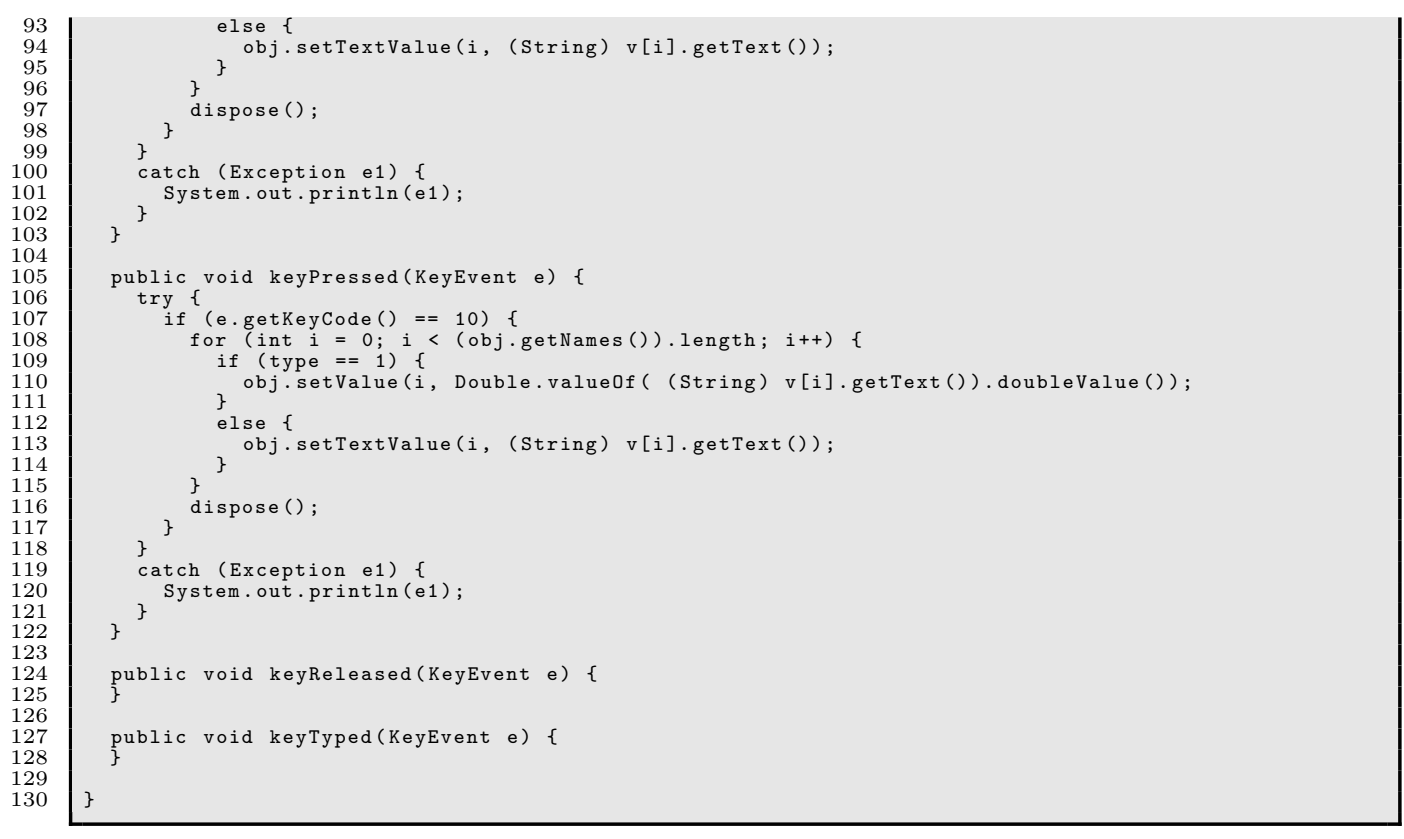




\section{B.18 XSelectItem.java}

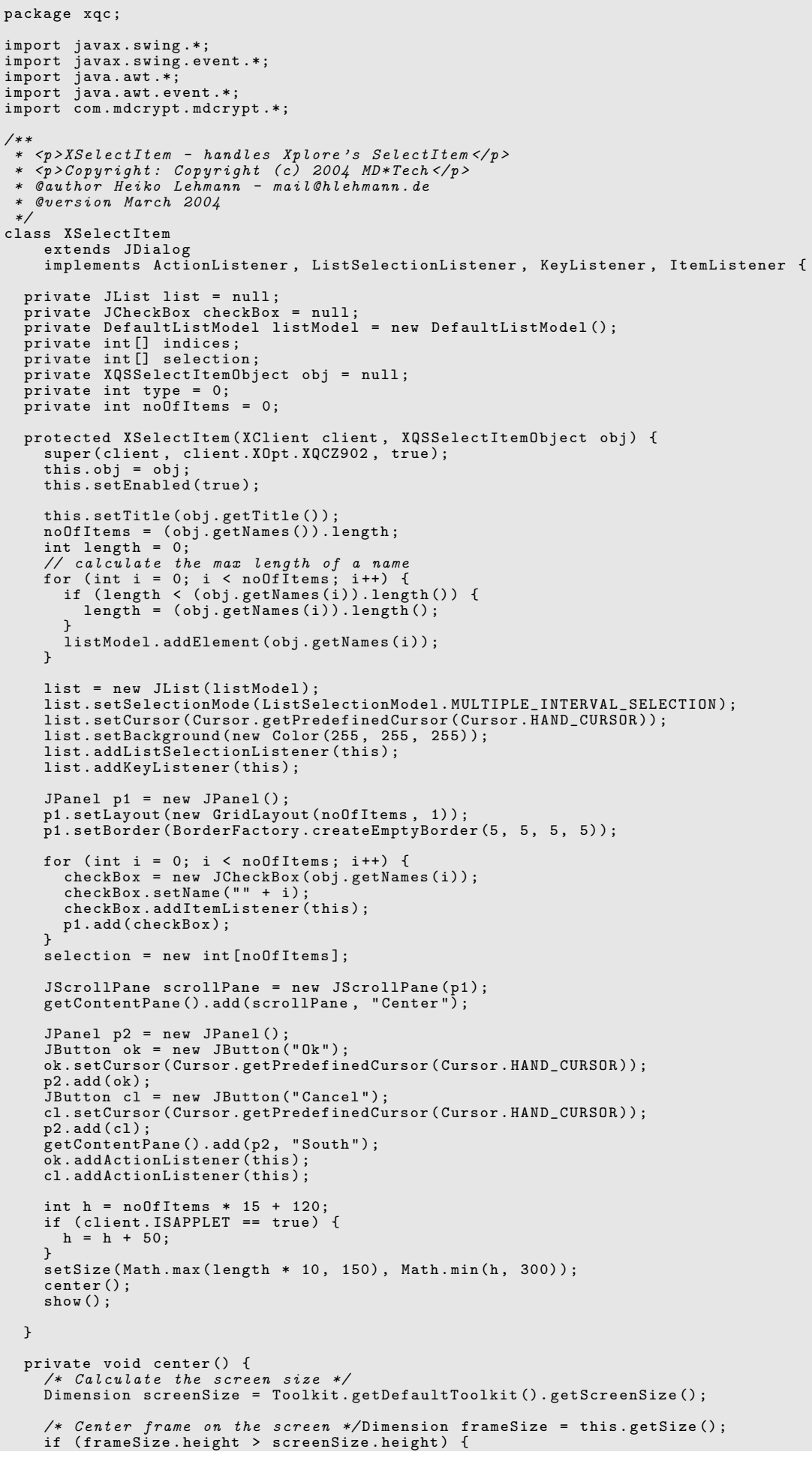




\section{B.18 XSelectItem.java}

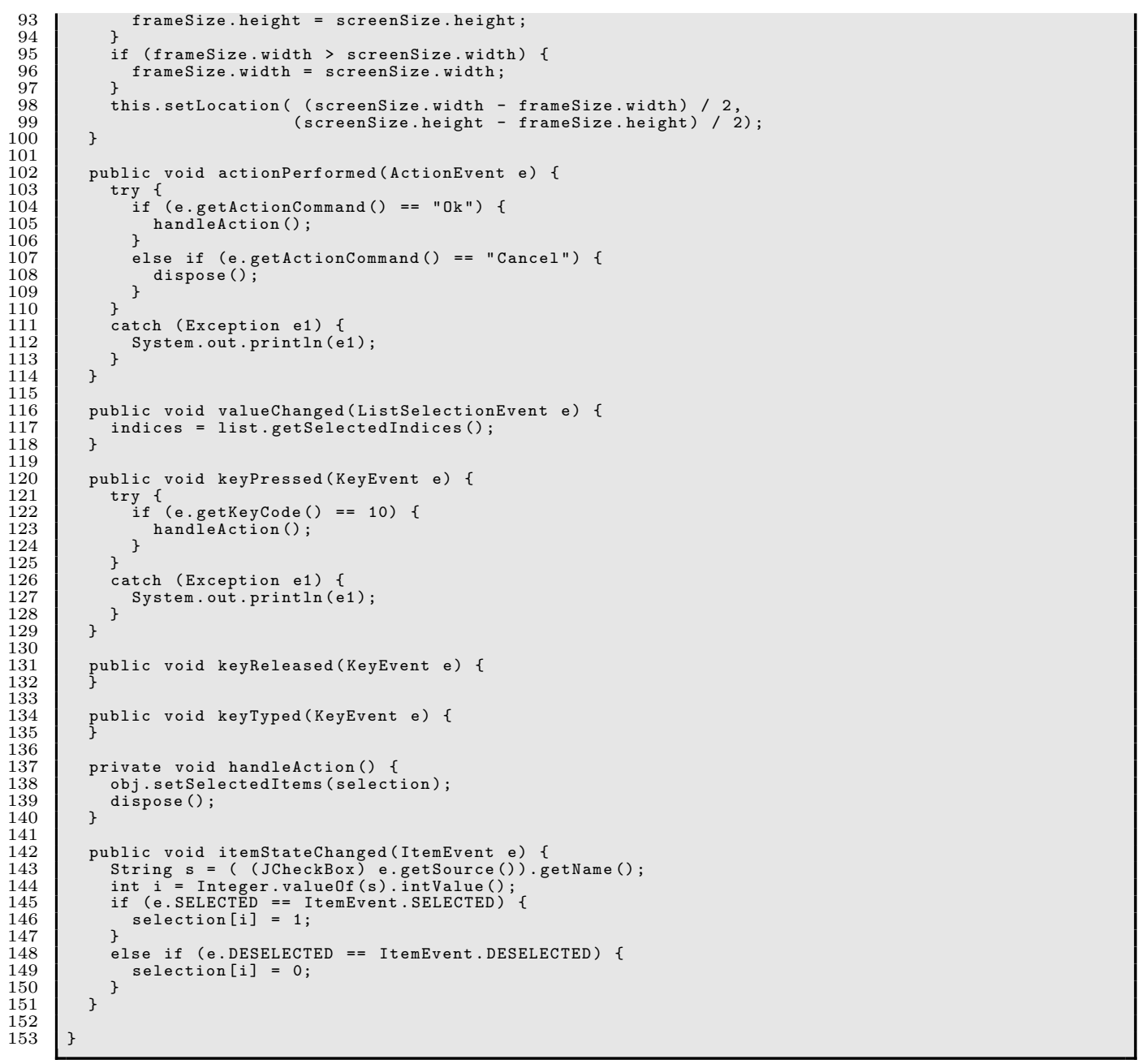


XQC Source Code 


\section{Appendix C}

\section{Example of a Third Party Client}

The following class is not part of the XQC. It just shows how the plot classes can easily be used within a third party program.

\section{C.1 ThirdPartyClient.java}

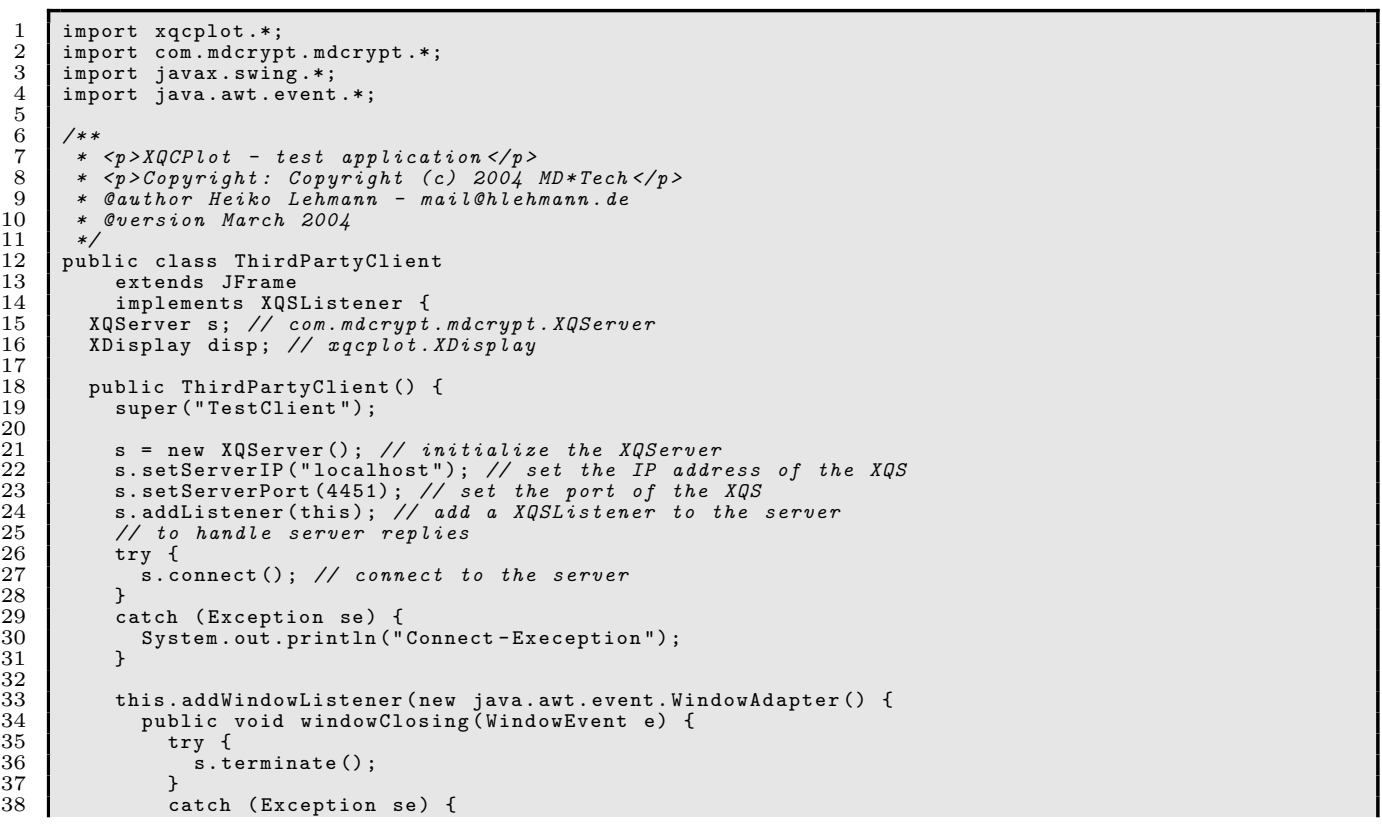




\section{Example of a Third Party Client}

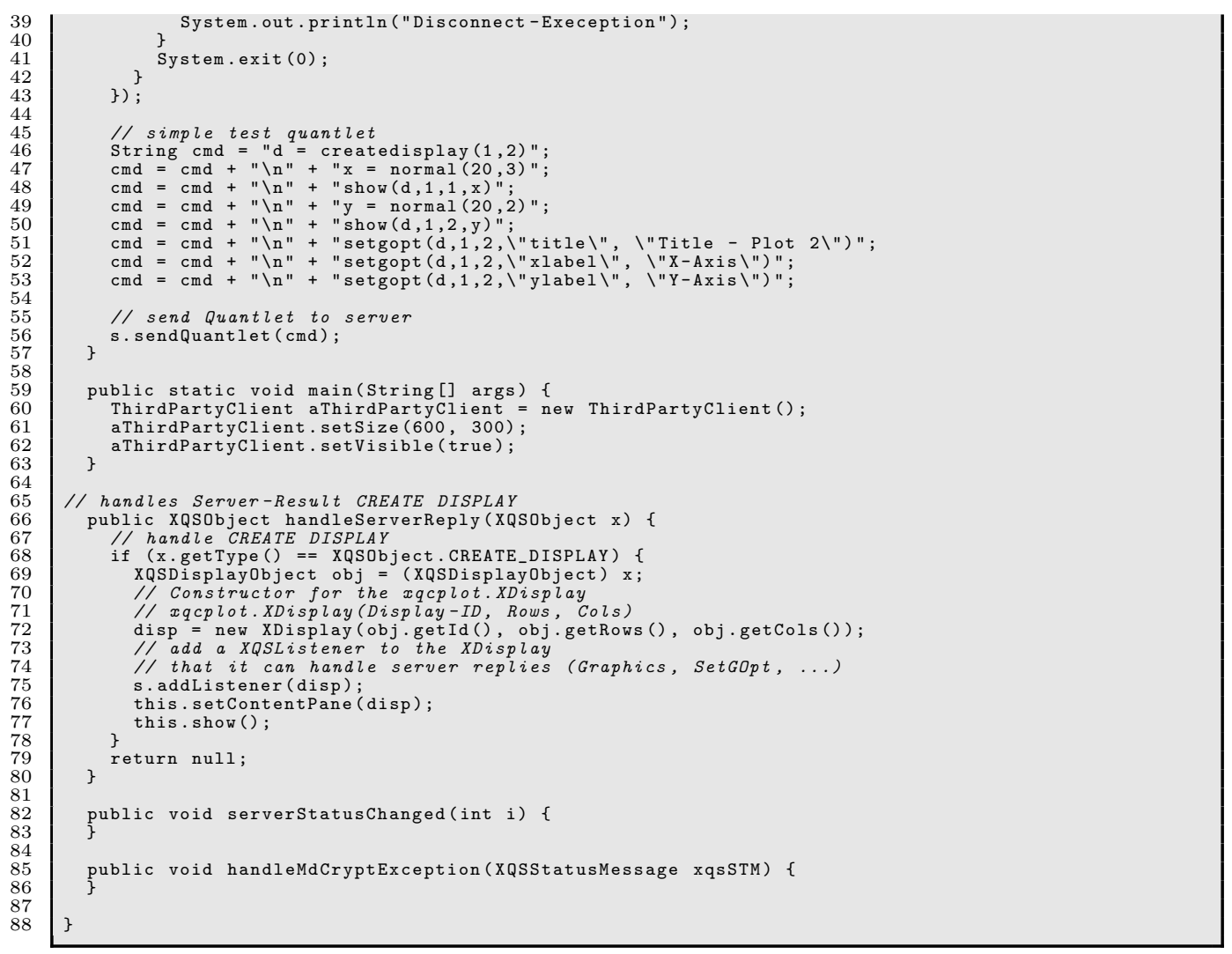




\section{Erklärung}

Hiermit erkläre ich, die vorliegende Arbeit selbständig und ohne fremde Hilfe verfasst und nur angegebene Literatur und Hilfsmittel verwendet zu haben. Personen, von denen ich Unterstützung erhalten habe, sind in der Danksagung genannt. Die vorliegende Dissertation war weder vollständig noch in Teilen Gegenstand einer früheren Begutachtung.

Ich bezeuge durch meine Unterschrift, dass meine Angaben über die bei der Abfassung meiner Dissertation benutzten Hilfsmittel, über die mir zuteil gewordene Hilfe sowie über frühere Begutachtungen meiner Dissertation in jeder Hinsicht der Wahrheit entsprechen.

Heiko Lehmann

02. April 2004 